University of Louisville

ThinkIR: The University of Louisville's Institutional Repository

Electronic Theses and Dissertations

$12-2019$

\title{
Laser spectroscopic investigations of organic alkoxy and peroxy radicals.
}

Md Asmaul Reza

University of Louisville

Follow this and additional works at: https://ir.library.louisville.edu/etd

Part of the Physical Chemistry Commons

\section{Recommended Citation}

Reza, Md Asmaul, "Laser spectroscopic investigations of organic alkoxy and peroxy radicals." (2019). Electronic Theses and Dissertations. Paper 3329.

https://doi.org/10.18297/etd/3329

This Doctoral Dissertation is brought to you for free and open access by ThinkIR: The University of Louisville's Institutional Repository. It has been accepted for inclusion in Electronic Theses and Dissertations by an authorized administrator of ThinkIR: The University of Louisville's Institutional Repository. This title appears here courtesy of the author, who has retained all other copyrights. For more information, please contact thinkir@louisville.edu. 


\title{
LASER SPECTROSCOPIC INVESTIGATIONS OF ORGANIC ALKOXY AND PEROXY RADICALS
}

\author{
By \\ Md Asmaul Reza \\ A Dissertation \\ Submitted to the Faculty of the \\ College of Arts and Sciences of the University of Louisville \\ in Partial Fulfillment of the Requirements \\ for the Degree of \\ Doctor of Philosophy in Chemistry
}

Department of Chemistry

University of Louisville

Louisville, Kentucky

December 2019 
Copyright by

Md Asmaul Reza

2019

All rights reserved 



\title{
LASER SPECTROSCOPIC INVESTIGATIONS OF ORGANIC ALKOXY AND PEROXY RADICALS
}

\author{
Md Asmaul Reza \\ A Dissertation Approved on \\ October 31, 2019 \\ by the following Dissertation Committee: \\ Dissertation Director - Dr. Jinjun Liu \\ Dr. Richard J. Wittebort
}

Dr. Lee Thompson

Dr. Sergio B. Mendes 
This dissertation is dedicated to my parents and my lovely wife 


\section{ACKNOWLEDGEMENTS}

It's been a dream come true for me to obtain a Ph.D. degree finally. The last six years in the chemistry department here at the University of Louisville has been one of the most fruitful experiences, both inside and outside of the laboratory, I have had in my life. I would like to thank the department for having faith in me and admitting me into the Ph.D. program.

First, I would like to express my gratitude to my advisor, Jinjun Liu, for his constant guidance and support and for giving me training in science beyond my imagination. His time and patience were really helpful in achieving my goals. I need to also acknowledge my dissertation defense committee members, Dr. Richard J. Wittebort, Dr. Lee Thompson, and Dr. Sergio B. Mendes. Thanks for being there in the key moment of my graduate school career. Next, I have to thank Neil J. Reilly to whom I owe a lot of what I know about the laser-induced fluorescence and dispersed fluorescence system. It was a wonderful experience to work with all the other members of the Liu group, specially Opu, and Hamzeh. I also want to acknowledge the incredible support from the staff, especially Steve, Sherry, Carla, Ben, Jason, and Renu, in the Department of Chemistry. Your kindness and care towards me have been very welcoming during my time here at UofL. 
I thank my parents, who always encouraged me and took great pride in my progress in this endeavor. Outside of my lab, I am very much indebted to my dearest friend Dhruba Kumar Pattadar for his timely help and invaluable suggestions during my Ph.D. course work. I am also grateful to my brother and their families for their support and advice in every possible way. My in-laws have stood by me always. I thank them for their encouragement. Finally, I would like to thank my beloved wife, Rumpa, for being with me and her encouragement and support to pursue my Ph.D. degree. 


\title{
ABSTRACT \\ LASER SPECTROSCOPIC INVESTIGATIONS OF ORGANIC ALKOXY AND PEROXY RADICALS
}

\author{
Md Asmaul Reza
}

October 31, 2019

Understanding the mechanism of combustion reactions is key for advancing internal combustion engine design, improving the efficiency of ignition chemical reactions, and reducing pollutant formation. In order to model the extremely complicated chemical kinetics of combustion processes, it is critical to detect and identify transient reactive chemical intermediates, particularly free radicals. Hence, it needs to be fully understood the gas-phase chemistry of organic compounds in the lower atmosphere. Over the past fifty years, parallel advancement in observational, experimental, and theoretical techniques, tremendous strides have been made in our understanding of the role of organic compounds in the atmosphere. Combustion chemistry is extremely complex, partly because of the large number of reaction intermediates that are involved in the process. Here we propose laser spectroscopic investigations of oxygen-containing combustion intermediates such as alkoxy (RO') and peroxy (ROO') radicals. These investigations are aimed at elucidating 
the structures and dynamics of free radicals, which are generated by laser photolysis of judiciously chosen precursors in a flow cell and supersonic jet expansion.

Spectroscopic interrogation of these species will be carried out using laser-induced fluorescence (LIF) spectroscopy, disperse fluorescence (DF), and cavity ring-down (CRD) spectroscopy. Vibrational structure of excited electronic states has been recorded using LIF and UV/visible/near-IR CRDS techniques, whereas DF and mid-IR CRDS techniques can be used to explore the vibrational structure of ground electronic states. Vibronic analyses were performed for the experimental spectra with the aid of quantum calculations. Accurate transition origin frequencies, excited and ground state vibrational frequencies, and FrankCondon factors were determined for different intermediates such as isobutoxy, 2-methyl1-butoxy, isopentoxy, 1-,2-,3-, and 4-methylcycholohexoy, and 1-,2-, and 3-methyl allyl peroxy. 


\section{TABLE OF CONTENTS}

PAGE

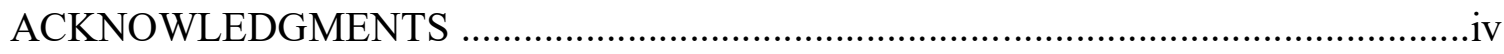

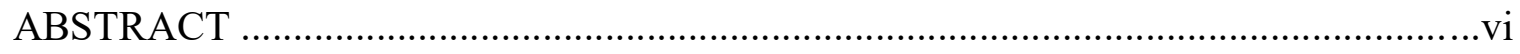

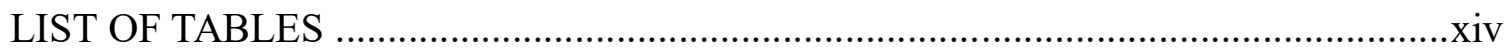

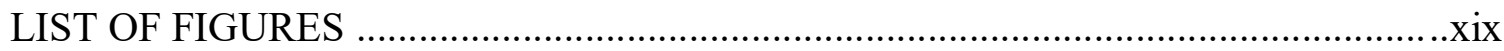

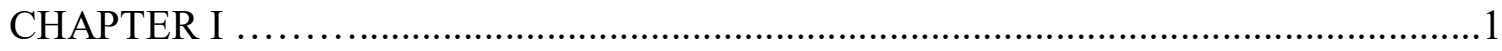

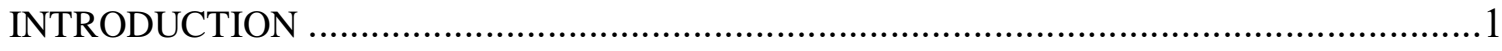

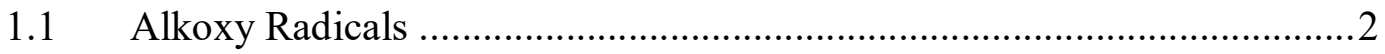

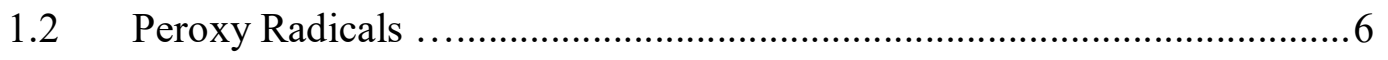

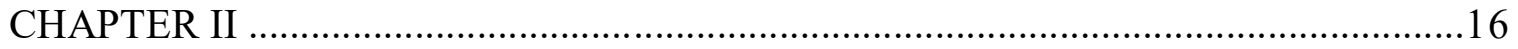

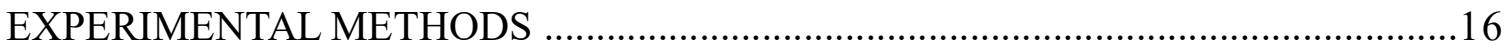

2.1. Laser-Induced Fluorescence (LIF) and Dispersed Fluorescence (DF)

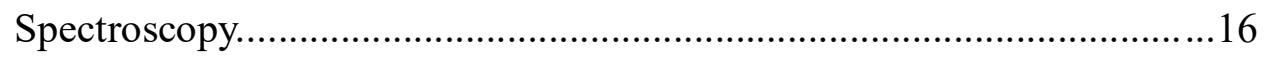

2.1.1 Introduction................................................................ 16

2.1.2 Moderate Resolution LIF Experimental Setup...........................17 
2.1.3 Moderate Resolution DF Experimental Setup................................18

2.1.4 Alkoxy Radical Production ........................................................18

2.2 Cavity Ring-Down Spectroscopy (CRDS)...............................................20

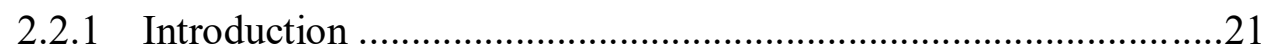

2.2.2 Room Temperature Experimental Setup........................................24

2.2.3 Peroxy Radical Production ..........................................................27

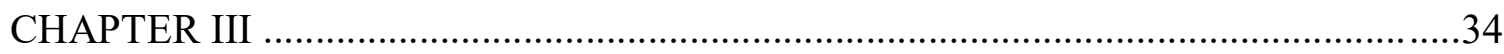

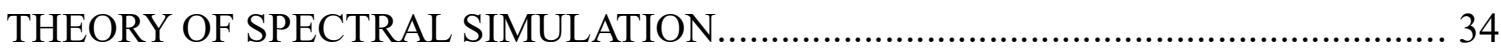

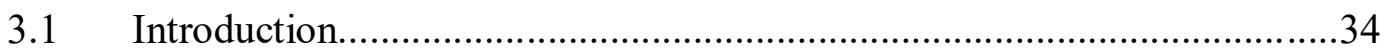

3.2 Energy Level Structure of Polyatomic Molecules.......................................35

3.2.1 Born-Oppenheimer (BO) Approximation......................................35

3.2.2 ab initio Computations...................................................................36

3.2.2 Molecular Vibrations......................................................................

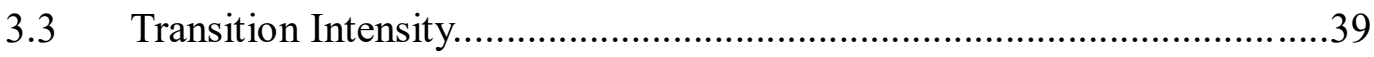

3.3.1 Separation of the electronic transition dipole moment and the

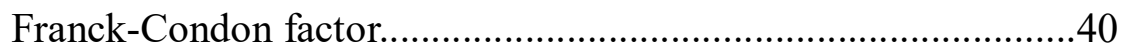

3.3.2 Franck-Condon factor.............................................................. 41

3.3.3 Computation of Frank-Condon factors and Duschinsky Mixing...42 ix 
CHAPTER IV

DISPERSED FLUORESCENCE SPECTROSCOPY OF JET-COOLED ISOBUTOXY

AND 2METHYL-1-BUTOXY RADICALS. 47

4.1 Introduction. 47

4.2 Quantum Chemical Calculations.. 50

4.3 Results. .51

4.3.1 Isobutoxy........ .51

4.3.1.1 Conformational Assignment .51

4.3.1.2 Vibrational Assignment. .52

4.3.1.3 $\tilde{A}-\tilde{X}$ Separation .54

4.3.2 2-Methyl-1-Butoxy....... .55

4.4 Discussion. .56

4.5 Conclusions. .60

CHAPTER V. .78

LASER-INDUCED FLUORESCENCE AND DISPERSED FLUORESCENCE SPECTROSCOPY OF JET-COOLED ISOPENTOXY RADICALS..... .78

5.1 Introduction. .78 
5.2 Calculations.

5.2.1 Potential Energy Surface (PES) Scan and Conformational

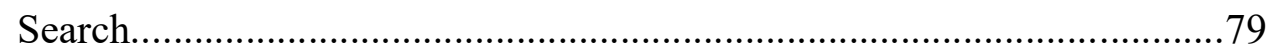

5.2.2 Quantum Chemical Calculations..................................................80

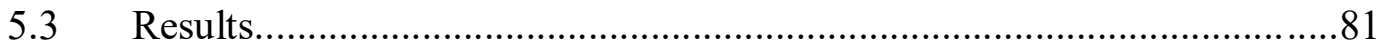

5.3.1 Overview of LIF and DF Spectra................................................

5.3.2 Simulation, Conformational Identification, and Vibrational

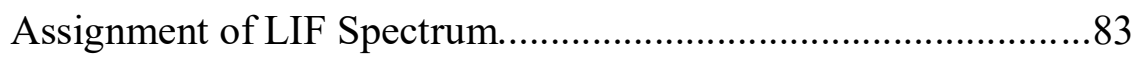

5.3.3 DF Spectra Obtained by Pumping the Origin Bands.....................85

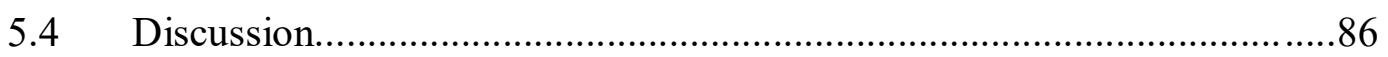

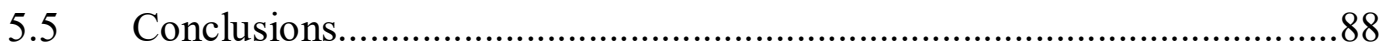

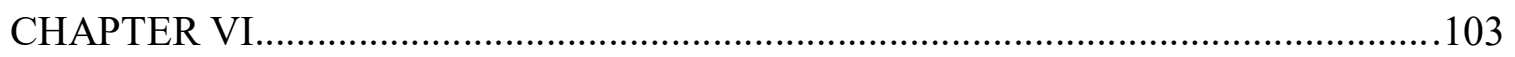

DISPERSED FLUORESCENCE SPECTROSCOPY OF JET-COOLED 2-, 3-, AND 4-

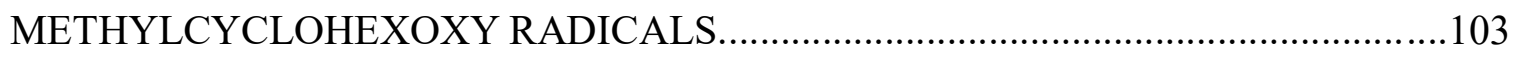

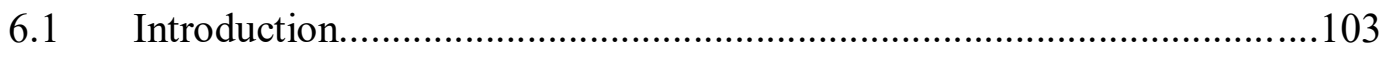

6.2 Quantum Chemical Calculations..........................................................105

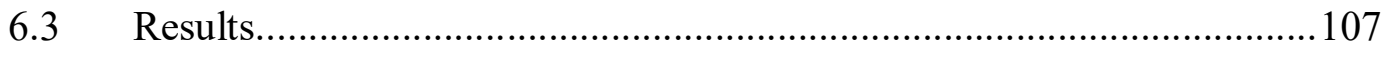

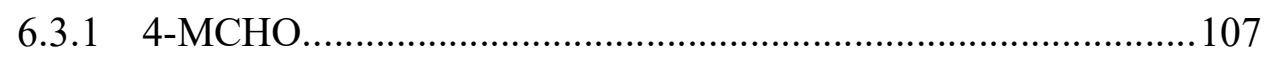


6.4 Discussion

6.4.1 Conformational and Vibrational Assignments.

6.4.2 Symmetry of $\tilde{A} / \tilde{X}$-States and Transition Intensities

6.4.3 $\tilde{A}-\tilde{X}$ Separation

6.5 Conclusions.

CHAPTER VII 148

DISPERSED FLUORESCENCE SPECTROSCOPY OF JET-COOLED 1METHYLCYCLOHEXOXY RADICALS. 148

7.1 Introduction. 148

7.2 Quantum Chemical Calculations. 149

7.3 Results. .150

6.3.1 Chair-Axial Conformer. 151

6.3.2 Chair-Equatorial Conformer.

7.4 Discussion. .155

7.5 Conclusions. 158 


\section{ROOM-TEMPERATURE CAVITY RING-DOWN SPECTROSCOPY OF}

METHYLALLYL PEROXY RADICALS ......................................................... 170

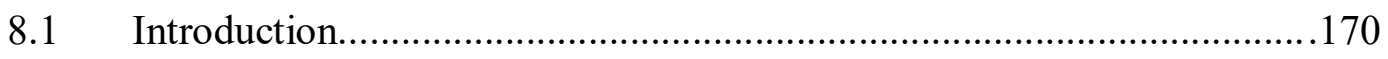

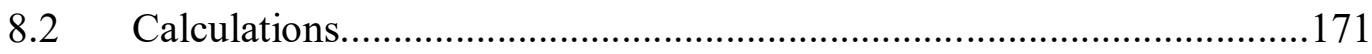

8.2.1 PES Survey and Conformational Search............................. 172

8.2.2 Quantum Chemical Calculations ........................................174

8.2.3 Spin Densities of 1-MA Radial and PES Scan Along the Reaction Coordinate of Oxygen Addition 174

8.3 Spectral Simulation and Assignment............................................. 175

8.3.1 Relative Populations of MAOO` Isomers ................................175

8.3.2 Population Ratios of Different Conformers of Each Isomer........176

8.3.3 Degeneracy of Each Conformer............................................176

8.3.4 Oscillator Strength of the Electronic Transition for Each Conformer 176

8.3.5 Franck-Condon Factors (FCFs) of the Vibronic Transitions.......177

8.3.6 Simulation of CRD Spectrum of 2-MAOO 178

8.3.6 Simulation of CRD Spectra of 1-MAOO and 3-MAOO 179 


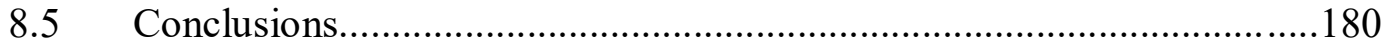

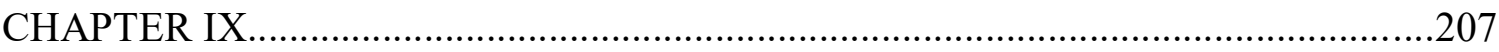

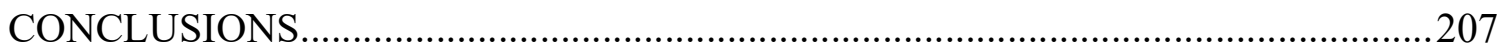

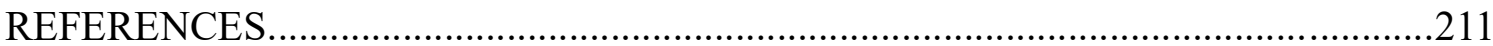

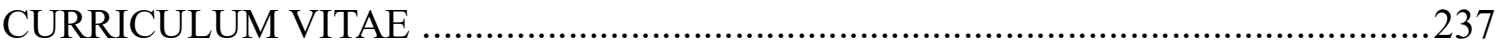




\section{LIST OF TABLES}

Table 4.1. Vibronic bands in the LIF spectra of isobutoxy and 2-methyl-1-butoxy reported in reference [29] and their assignments based on DF spectra and calculations obtained in the present work

Table 4.2. Assignment of transitions in the DF spectrum of the $C_{s}$ conformer of the isobutoxy radical by pumping its origin band A (Figure 4.1)

Table 4.3. Assignment of transitions in the DF Spectrum of the $C_{1}$ conformer of the isobutoxy radical by pumping its origin band B (Figure 4.1 )

Table 4.4. Assignment of transitions in the DF spectrum of the $\mathrm{T}_{1} \mathrm{~T}_{2}$ conformer of the 2methyl-1-butoxy radical by pumping its origin band A(Figure 4.4)

Table 4.5. Assignment of transitions in the DF spectrum of the $\mathrm{G}_{1} \mathrm{~T}_{2}$ conformer of the 2methyl-1-butoxy radical by pumping its origin band B (Figure 4.4) .65

Table 4.6. Experimentally determined ground-state vibrational energy levels $\mathrm{G}(v)$ of the CO stretch progression (in $\mathrm{cm}^{-1}$ ) of the $C_{1}$ conformer of the isobutoxy radical. $\Delta G(v)=$ $G(v)-G(v-1)$ .66

Table 4.7. Experimentally determined ground-state vibrational energy levels $G(v)$ of the $\mathrm{CO}$ stretch progression $\left(\mathrm{cm}^{-1}\right)$ of $\mathrm{G}_{1} \mathrm{~T}_{2}$ conformer of the 2-methyl-1-butoxy radical .66 
Table 5.1. Geometric parameters of conformers of the isopentoxy radical in the $\tilde{X}$ - and $\tilde{B}$ states calculated at the CAM-B3LYP/6-311+G(d) level of theory

Table 5.2. Numbers of enantiomers calculated relative energies, adiabatic excitation energies $\left(\Delta E^{\tilde{B}-\tilde{X}}\right)$ and oscillator strengths $(f)$ of the $\tilde{B}-\tilde{X}$ transition, and $\tilde{X}$ - and $\tilde{B}$-state COstretch frequencies $\left(\nu_{\mathrm{CO}}^{\tilde{\mathrm{X}}}\right.$ and $\left.\nu_{\mathrm{CO}}^{\tilde{\mathrm{B}}}\right)$ of isopentoxy conformers. $\Delta E^{\tilde{B}-\tilde{X}}$ includes zero-pointenergy corrections. Note that for the TG and GG conformers, there is more than one vibrational mode with the $\mathrm{CO}$-stretch character.

Table 5.3. Experimental and calculated vibrational frequencies $\left(\mathrm{cm}^{-1}\right)$ and assignment of vibronic bands in the LIF spectra (Figure 5.2a). .91

Table 6.1. Redshifts ("rs") from the pumped LIF bands (A-F) and relative wavenumbers ("rel") observed in the DF spectra of 4-MCHO $\left(\mathrm{cm}^{-1}\right)$ 125

Table 6.2. Redshifts ("rs") from the pumped LIF bands $\left(\mathrm{A}-\mathrm{C}, \mathrm{A}^{*-} \mathrm{C}^{*}\right)$ and relative wavenumbers ("rel") observed in the DF spectra of 3-MCHO $\left(\mathrm{cm}^{-1}\right)$

Table 6.3. Redshifts ("rs") from the pumped LIF bands (A-F) and relative wavenumbers ("rel") observed in the DF spectra of 2-MCHO $\left(\mathrm{cm}^{-1}\right)$

Table 6.4. Calculated vibrational frequencies $\left(\mathrm{cm}^{-1}\right)$ of $\tilde{X}$-state 4-MCHO (with pseudoJahn-Teller distorted geometry) and Franck-Condon factors (FCFs) for transitions from the vibrational ground level of its $\tilde{B}$-state to $\tilde{X}$-state fundamental vibrational levels. (Threshold for FCFs is 0.001.) 
Table 6.5. Calculated vibrational frequencies $\left(\mathrm{cm}^{-1}\right)$ of $\tilde{A}^{2} \mathrm{~A}^{\prime}$ state 4-MCHO and FranckCondon factors (FCFs) for transitions from the vibrational ground level of its $\tilde{B}^{2} \mathrm{~B}^{\prime}$ state to $\tilde{A}^{2} \mathrm{~A}^{\prime}$ state fundamental vibrational levels. (Threshold for FCFs is 0.001.) 133

Table 6.6. Calculated vibrational frequencies $\left(\mathrm{cm}^{-1}\right)$ of $\tilde{X}$-state 3-MCHO and FranckCondon factors (FCFs) for transitions from the vibrational ground level of its $\tilde{B}$-state to $\tilde{X}$ state fundamental vibrational levels. (Threshold for FCFs is 0.001.) 135

Table 6.7. Calculated vibrational frequencies $\left(\mathrm{cm}^{-1}\right)$ of $\tilde{X}$-state 2-MCHO and FranckCondon factors (FCFs) for transitions from the vibrational ground level of its $\tilde{B}$-state to $\tilde{X}$ state fundamental vibrational levels. (Threshold for FCFs is 0.001.)

Table 7.1. Numbers of conformers and their calculated relative energies, energy separation between $\tilde{A}$ - and $\tilde{X}$-states $\left(\Delta E^{\tilde{A}-\tilde{X}}\right)$ and $\tilde{X}$ - and $\tilde{A}$-state $\mathrm{CO}$-stretch and $\mathrm{C}_{-} \mathrm{CH}_{3}$-stretch frequencies of 1-MCHO conformers. $\Delta E^{\tilde{A}-\tilde{X}}$ includes zero-point-energy corrections $\ldots .160$ Table 7.2. Redshifts (r.s.) from the pumped LIF lines and relative wavenumbers (rel.) observed in the DF spectra of the chair-axial conformer of 1-MCHO $\left(\mathrm{cm}^{-1}\right)$.

Table 7.3. Redshifts (r.s.) from the pumped LIF lines and relative wavenumbers (rel.) observed in the DF spectra of the chair-equatorial conformer of 1-MCHO $\left(\mathrm{cm}^{-1}\right)$.

Table 8.1. Numbers of (optically distinguishable) conformations, transition structures, and stable conformers of 1-, 2- and 3-MAOO radicals. 
Table 8.2. Relative energies, Boltzmann distribution factors, and relative populations of 1and 3-MAOO conformers. $\Delta E^{\tilde{A}-\tilde{X}}$ is the adiabatic transition frequency between the $\tilde{X}$ - and $\tilde{A}$-states. $v_{\mathrm{OO}}(\tilde{A})$ is the $\tilde{A}$-state $\mathrm{OO}$ stretch frequency.

Table 8.3. Relative energies, Boltzmann distribution factors, and relative populations of 2MAOO conformers. $\Delta E^{\tilde{A}-\tilde{X}}$ is the adiabatic transition frequency between the $\tilde{X}$ - and $\tilde{A}$ states. $v_{\mathrm{OO}}(\tilde{A})$ is the $\tilde{A}$-state $\mathrm{OO}$ stretch frequency. 184

Table 8.4. Geometric parameters of conformers of 1-, 2- and 3- MAOO radicals in the $\tilde{X}$ and $\tilde{A}$ - states calculated at the B3LYP/6-31+G(d,p) level of theory. 185 


\section{LIST OF FIGURES}

Figure 1.1. Schematic diagram for combustion of hydrocarbons taken from Ref. [8].

Figure 1.2. Mechanism of peroxy radical formation from $\mathrm{OH}$-initiated oxidation of isoprene. The allyl peroxy functionality (highlighted in red) is achieved in four of the six possible addition sequences taken from Ref. [72]. 14

Figure 1.3. Methyl peroxy potential energy surfaces of the $\tilde{X}$-, $\tilde{A}$-, and $\tilde{B}$-states as a function of Roo taken from Ref. [83].

Figure 2.1. Moderate-resolution LIF/DF apparatus.

Figure 2.2. Supersonic cooling. 30

Figure 2.3. Principle of cavity ring-down spectroscopy.

Figure 2.4. A schematic of a room temperature CRDS apparatus.

Figure 2.5. Schematics of production of (a) 2-MAOO radical using 3-chloro-2-methyl-1propene as the precursor, and 1- and 3-MAOO radicals using either (b) 1-chloro-2-butene or (c) 3-chloro-1-butene as the precursor. Bidirectional arrows denote allylic rearrangement.

Figure 3.1. Franck-Condon principle energy diagram taken from Ref [119]. Since electronic transitions are very fast compared with nuclear motions, vibrational levels are 
favored when they correspond to a minimal change in the nuclear coordinates. The potential wells are shown favoring transitions between $v=0$ and $v=2$.

Figure 3.2. Duschinsky mixing between normal modes can be presented as a rotation of the vibrational normal coordinates. $Q=\frac{1}{2 \pi} \sqrt{\frac{2 h}{v \mu}\left(v+\frac{1}{2}\right)}$, where $Q$ "' and $Q$ ' are the normal coordinates of the ground state and the excited state, respectively. $h$ is the Planck's constant, $\mu$ is the reduced mass, and $v$ is the vibrational quantum number. .46

Figure 4.1. DF spectra of the isobutoxy radical. The asterisked peak is due to the scattering of the $355 \mathrm{~nm}$ photolysis laser. Numbers in parentheses are LIF excitation frequencies....67

Figure 4.2. Comparison between the simulated DF spectra of the Cs and C1 conformers of the isobutoxy radical (blue) and the experimentally obtained ones by pumping bands A and B in the LIF spectrum (red).

Figure 4.3. DF spectra of the isobutoxy radical obtained by pumping LIF bands B-D. The spectra obtained by pumping vibronic bands other than the origin (band B) are blue-shifted by the $\tilde{X}$-state vibrational frequency of the pumped mode so that the DF bands to the $\tilde{X}$ state vibrational levels are aligned.

Figure 4.4. DF spectra of 2-methyl-1-butoxy radical (red) compared with simulation (blue). The asterisked peak is due to the scattering of the $355 \mathrm{~nm}$ photolysis laser. .70

Figure 4.5. Comparison between the second members of the $\mathrm{CO}$-stretch progression in the DF spectra of the isobutoxy radical by pumping Bands A and B in the LIF spectrum. Difference in transition frequencies of the observed DF peaks supports a two-conformer 
assignment.

Figure 4.6. Determination of the harmonic frequency $\left(\omega_{e}\right)$ and the anharmonic parameter $\left(\omega_{e} \chi_{e}\right)$ of the $\tilde{X}$-state CO stretch of the $C_{1}$ conformer of isobutoxy. $G(v)$ is the vibrational energy of the $v^{\text {th }}$ member of the CO-stretch progression.

Figure 4.7. Vibrational modes of the $C_{1}$ conformer of isobutoxy. a) $-\mathrm{CH}_{2} \mathrm{O}$ wagging mode (v36), to which Bands C (in the LIF spectrum) and c (in the DF spectra) are assigned. b) inphase $\mathrm{CH}_{3}$ torsional mode $\left(v_{34}\right)$, to which Bands $\mathrm{D}$ (in the LIF spectrum) and $\mathrm{d}$ (in the DF spectra) are assigned. In parentheses are calculated vibrational frequencies in $\mathrm{cm}^{-1}$. Unprimed and primed labels are used to denote the $\tilde{X}$ - and $\tilde{B}$-state vibrational modes, respectively. 73

Figure 4.8. $\tilde{X}$-state vibrational modes of the $C_{s}$ conformer of isobutoxy. a) $v_{16}$ (umbrella mode), b) $v_{15}$ (mixture of $\mathrm{CH}_{3}$ scissoring and $\mathrm{C}-\mathrm{C}$ stretch modes), c) $v_{14}$, and d) $v_{13}, v_{14}$ and $v_{13}$ are mixtures of $\mathrm{CO}$-stretch and the umbrella modes.

Figure 4.9. Determination of the harmonic frequency $\left(\omega_{e}\right)$ and the anharmonic parameter $\left(\omega_{e} \chi_{e}\right)$ of the $\tilde{X}$-state CO-stretch of the $\mathrm{G}_{1} \mathrm{~T}_{2}$ conformer of 2-methyl-1-butoxy. .75

Figure 4.10. $\tilde{X}$-state vibrational modes of the $\mathrm{T}_{1} \mathrm{~T}_{2}$ conformer of 2-methyl-1-butoxy. a) $v_{43}$ (C-C-C bending mode), b) $v_{30}$, and c) $v_{28}, v_{30}$ and $v_{28}$ are mixtures of $\mathrm{CO}$-stretch and the $\mathrm{C}$ C stretch modes. .76

Figure 4.11. $\tilde{X}$-state vibrational modes of the $\mathrm{G}_{1} \mathrm{~T}_{2}$ conformer of 2-methyl-1-butoxy radical. a) $v_{43}\left(\mathrm{C}_{3}\right.$-position methyl torsional mode $)$, b) $v_{42}\left(\mathrm{C}_{4}\right.$-position methyl torsional mode $)$ and c) $v_{28}(\mathrm{CO}$-stretch mode). .76 
Figure 4.12. DF spectra of the isobutoxy radical by pumping Band E in its LIF spectrum, which suggests that the molecular carrier of Band $\mathrm{E}$ is formaldehyde. .77

Figure 5.1. The five conformers of isopentoxy and their relative energies $\left(\mathrm{cm}^{-1}\right)$. .92

Figure 5.2. (a) Vibrationally resolved LIF spectrum of isopentoxy. (b-d) Simulated LIF spectra of the GG' (b), GG (c) and TG (d) conformers. The asterisked band is due to $\mathrm{CH}_{2} \mathrm{O}$. .93

Figure 5.3. DF spectra of the isopentoxy radical obtained by pumping LIF bands $\mathrm{B}_{0}-\mathrm{B}_{4}$. Numbers in parentheses after the pumped LIF bands are relative wavenumbers with respect to the origin band (Band $\mathrm{B}_{0}$ ). The asterisked bands are due to photolysis laser scattering. 94

Figure 5.4. DF spectra of the isopentoxy radical obtained by pumping LIF bands C-L. Numbers in parentheses after the pumped LIF bands are relative wavenumbers with respect to the origin band (Band C). The asterisked bands are due to photolysis laser scattering. ..95

Figure 5.5. Birge-Sponer plot of the "A" Bands in the LIF spectrum of isopentoxy. .......96

Figure 5.6. Birge-Sponer plot of the "B" Bands in the LIF spectrum of isopentoxy

Figure 5.7. DF spectrum of the isopentoxy radical obtained by pumping LIF bands $A_{2}$. Numbers in parentheses after the pumped LIF bands are relative wavenumbers with respect to the origin band (Band $\mathrm{A}_{0}$ ). The asterisked band is due to photolysis laser scattering. ....98

Figure 5.8. DF spectra of the isopentoxy radical obtained by pumping LIF bands $\mathrm{B}_{0}-\mathrm{B}_{4}$. The DF spectra are blue-shifted by ground-state vibrational frequencies of the pumped modes. Numbers in parentheses after the pumped LIF bands are relative wavenumbers with 
respect to the origin band $\left(B\right.$ and $\left.\mathrm{B}_{0}\right)$. The asterisked band is due to photolysis laser scattering. .99

Figure 5.9. DF spectra of the isopentoxy radical obtained by pumping LIF bands C-L. The DF spectra except that obtained by pumping the CO stretch band (Band L) are blue-shifted by ground-state vibrational frequencies of the pumped modes. Numbers in parentheses after the pumped LIF bands are relative wavenumbers with respect to the origin band (Band C). The asterisked band is due to photolysis laser scattering.

Figure 5.10. Scans of $\tilde{X}$ - and $\tilde{B}$-state potential energy surfaces of the isopentoxy radical along the coordinates of the $\mathrm{OC}_{1} \mathrm{C}_{2} \mathrm{C}_{3}$ and $\mathrm{C}_{1} \mathrm{C}_{2} \mathrm{C}_{3} \mathrm{H}$ dihedral angles. .101

Figure 5.11. LIF spectrum that contains transitions of formaldehyde, a product of $\beta \mathrm{C}-\mathrm{C}$ fission of isopentoxy. 102

Figure 6.1. Half-filled orbitals of the lowest-energy conformers of (a) 2-MCHO, (b) 3$\mathrm{MCHO}$, and (c) 4-MCHO. Hydrogen atoms are not shown for clarity except the one at the $\mathrm{C}_{1}$-position. 138

Figure 6.2. DF spectra of 4-MCHO. Numbers in parentheses after the LIF bands are relative wavenumbers with respect to the origin band (band A) at $26906 \mathrm{~cm}^{-1}$.... 139

Figure 6.3. DF spectra of 2, 3-, and 4-MCHOs by pumping the LIF origin bands compared with calculated transition intensities and simulated spectra. Blue and red sticks represent $\tilde{B}$ $\rightarrow \tilde{X}$ and $\tilde{B} \rightarrow \tilde{A}$ transitions, respectively.

Figure 6.4. DF spectra of 3-MCHO. Numbers in parentheses after the LIF bands are relative wavenumbers with respect to the origin band (band A) at $26677 \mathrm{~cm}^{-1}$. 141 
Figure 6.5. Excited-state vibrational population relaxation (wavy arrows) and fluorescence emission (red arrows) after $\tilde{B} \leftarrow \tilde{X}$ excitation (black arrow) of 3-MCHO to CO-stretch combinations bands $\left(v_{\mathrm{CO}}+v^{*}\right) . \tilde{X}$ - and $\tilde{A}$-states are semi degenerate. Dashed horizontal lines represent vibrational energy levels of the $\tilde{X}$-state

Figure 6.6. DF spectra of 2-MCHO. Numbers in parentheses after the LIF bands are relative wavenumbers with respect to the origin band (band A) at $26275 \mathrm{~cm}^{-1}$.

Figure 6.7. DF spectra of 4-MCHO. The spectra by pumping vibronic bands other than the origin band (A) in the LIF spectrum are blueshifted by the $\tilde{A}$-state vibrational frequency of the pumped mode so that the DF bands to the $\tilde{A}$-state vibrational level of the pumped modes are aligned. 144

Figure 6.8. DF spectra of 3-MCHO. The spectra by pumping vibronic bands other than the origin band (A) and the CO stretch band $\left(A^{*}\right)$ in the LIF spectrum are blueshifted by the A-state vibrational frequency of the pumped mode so that the DF bands to the $\tilde{A}$-state vibrational level of the pumped modes are aligned. 145

Figure 6.9. DF spectra of 2-MCHO. The spectra by pumping vibronic bands other than the origin band (A) in the LIF spectrum are blueshifted by the $\tilde{A}$-state vibrational frequency of the pumped mode so that the DF bands to the $\tilde{A}$-state vibrational level of the pumped modes are aligned. 146

Figure 6.10. $\tilde{B}$-state vibrational mode $v_{54}$ of $2-\mathrm{MCHO}$. 147 
Figure 7.1. DF spectra of the chair-axial conformer of 1-MCHO. Numbers in parentheses after the LIF bands are relative wavenumbers with respect to the origin band (Band D) at $27614 \mathrm{~cm}^{-1}$ 163

Figure 7.2. DF spectrum of band D $\left(27614 \mathrm{~cm}^{-1}\right)$ in the LIF of the chair-axial conformer of 1-MCHO. The asterisks are the assignments of the DF spectrum, $v_{\mathrm{C}-\mathrm{CH} 3}(\tilde{X}), v_{\mathrm{C}-\mathrm{CH} 3}(\tilde{A}$ ) , and $v_{\mathrm{C}-\mathrm{CH} 3}(\tilde{X})+v_{\mathrm{CO}}(\tilde{X})$, respectively. 164

Figure 7.3. DF spectra of the chair-equatorial conformer of 1-MCHO. Numbers in parentheses after the LIF bands are relative wavenumbers with respect to the origin band (Band B) at $27455 \mathrm{~cm}^{-1}$. 165

Figure 7.3. DF spectrum of band B $\left(27455 \mathrm{~cm}^{-1}\right)$ in the LIF spectrum of the chair-equatorial conformer of 1-MCHO. The asterisks are the assignments of the DF spectrum, $v_{\mathrm{C}-\mathrm{CH} 3}(\tilde{X})$, $v_{\mathrm{C}-\mathrm{CH} 3}(\tilde{A})$, and $v_{\mathrm{C}-\mathrm{CH} 3}(\tilde{X})+v_{\mathrm{CO}}(\tilde{X})$, respectively. 166

Figure 7.5. DF spectra of unknown LIF bands of 1-MCHO. Numbers in parentheses are the LIF frequencies. 167

Figure 7.6. Comparison between the experimental (bottom red trace) and simulated (top black trace) DF spectra of the chair-axial conformer of the 1-MCHO radical. The experimental DF spectra were obtained by pumping the origin band in the LIF spectrum (Band D). .168

Figure 7.7. Comparison between the experimental (bottom red trace) and simulated (top black trace) DF spectra of the chair-equatorial conformer of the 1-MCHO radical. The 
experimental DF spectra were obtained by pumping the origin band in the LIF spectrum (Band B).

Figure 8.1. Schematics of production of (a) 2-MAOO radical using 3-chloro-2-methyl-1propene as the precursor, and 1- and 3-MAOO radicals using either (b) 1-chloro-2-butene or (c) 3-chloro-1-butene as the precursor. Bidirectional arrows denote allylic rearrangement. 186

Figure 8.2. Schematics of 1-, 2- and 3-MAOO radicals are showing the convention used to label carbon atoms in the present work. 187

Figure 8.3. Plots of spin densities of $\tilde{X}$-state (a) cis-1-methylallyl and (b) trans-1methylallyl radicals calculated at the B3LYP/6-31+(d,p) level of theory. The regions in red and purple meshes represent excess $\alpha$ spin and $\beta$ spin, respectively. 188

Figure 8.4. PES scans for production of 1- and 3-MAOO isomers along the reaction coordinate of oxygen addition. $R_{\mathrm{C}} . .(\mathrm{OO})$ is the distance between the $\mathrm{C}_{3}$ atom and the center of mass of the $\mathrm{OO}$ moiety.

Figure 8.5. (a) Experimental CRD spectrum of the 2-MAOO radical (red) in comparison with simulation at the temperature of $300 K$ (green). Sharp peaks between 7300 and 7500 $\mathrm{cm}^{-1}$ in the experimental spectrum are due to residual water absorption. (b) Simulated spectrum of 2-MAOO . Thin lines are simulated spectra of individual conformers weighted by relative populations. The thick green line is superposition of simulated spectra of all conformers. 190 
Figure 8.6. (a) Experimental CRD spectrum of the 2-MAOO radical (red) in comparison with simulation at the temperature of $50 K$ (purple). (b) Simulated spectra of 2-MAOO at different temperatures. Overall spectra of 2-MAOO are calculated as summation of spectra of all conformers weighted by their relative populations assuming the Boltzmann distribution (not shown in the figure). All spectra are offset for clarity. The simulation with $\mathrm{T}=50 K$ (purple) reproduces the experimental spectrum the best. 191

Figure 8.7. CRD spectrum of the 1- MAOO and 3- MAOO radicals using either (a) 3chloro-1-butene (blue) or (b) 75\% 1-chloro-2-butene and 25\% 3-chloro-1-butene mixture (red) as precursors. Strong absorption with sharp features between 7300 and $7500 \mathrm{~cm}^{-1}$ is due to methyl peroxy. Experimental spectra are compared with simulated overall spectra of 1- and 3-MAOO radicals (green). (c) Simulated spectrum of 1-MAOO . (d) Simulated spectrum of 3-MAOO. Thin lines in (c) and (d) are simulated spectra of individual conformers weighted by populations, while the thick green line is their superposition. (e) Superposition of the simulated spectra of both isomers (green) with weights of $52 \%$ for 1 MAOO (red) and 48\% for 3-MAOO (blue).

Figure 8.8. Newman projections of 1-MAOO conformers.

Figure 8.9. Newman projections of 2-MAOO conformers. 194

Figure 8.10. Newman projections of 3-MAOO conformers.

Figure 8.11. A scan of the potential energy surface of 1-MAOO along the coordinate of the CCOO dihedral angle. Molecular geometry is optimized at each step of the scan. .196 
Figure 8.12. A scan of the potential energy surface of T 1-MAOO along the coordinate of the CCCO dihedral angle. Molecular geometry is optimized at each step of the scan. .197

Figure 8.13. A scan of the potential energy surface of 2-MAOO along the coordinate of the CCOO dihedral angle. Molecular geometry is optimized at each step of the scan. 198

Figure 8.14. A scan of the potential energy surface of 2-MAOO along the coordinate of the CCCO dihedral angle. Molecular geometry is optimized at each step of the scan.

Figure 8.15. A scan of the potential energy surface of 3-MAOO along the coordinate of the CCOO dihedral angle. Molecular geometry is optimized at each step of the scan. .200

Figure 8.16. A scan of the potential energy surface of 3-MAOO along the coordinate of the CCCO dihedral angle. Molecular geometry is optimized at each step of the scan. .201

Figure 8.17. Simulated spectra of 2-MAOO conformers. Overall spectrum of 2-MAOO is calculated as summation of spectra of all conformers weighted by their relative populations at $300 \mathrm{~K}$. All spectra are offset for clarity. .202

Figure 8.18. Simulated spectra of 1-MAOO conformers. Overall spectrum of 2-MAOO is calculated as summation of spectra of all conformers weighted by their relative populations at $300 \mathrm{~K}$. All spectra are offset for clarity. .203 
Figure 8.19. Simulated spectra of 3-MAOO conformers. Overall spectrum of 3-MAOO is calculated as summation of spectra of all conformers weighted by their relative populations at $300 \mathrm{~K}$. All spectra are offset for clarity.

Figure 8.20. CRD spectra of MAOO isomers (red) and their precursors: 3-chloro-2methyl-1-propene for 2-MAOO production (top), and 75\% 1-chloro-2-butene and 25\% 3chloro-1-butene mixture for 1- and 3-MAOO production (bottom). The free radical spectra are obtained by subtracting the "photolysis laser off" spectrum (black) from the "photolysis laser on" spectrum (blue). 205

Figure 8.21. (a) CRD spectrum of the 1- MAOO and 3-MAOO radicals. (b) Simulated spectrum of $52 \% 1-\mathrm{MAOO}+48 \% 3-\mathrm{MAOO}$ (c) Simulated spectra with different population ratios of 1- MAOO and 3- MAOO. Spectra are offset for clarity. .206 


\section{CHAPTER I}

\section{INTRODUCTION}

Laser spectroscopy is a powerful tool for the identification and diagnosis of chemical reaction intermediates. It has been arguably the pre-eminent tool for the study of molecular dynamics and chemical kinetics, which has greatly advanced our understanding of the electronic structure and chemical reactivity. However, there still exist vast, unexplored gaps in our knowledge that demand further investigation. A major experimental challenge for the molecular spectroscopist is the generation of target molecular species in sufficient quantities and under the appropriate conditions to permit their detailed study by highresolution methods. Particularly difficult are reactive species, mostly free radicals and molecular complexes. Different transient species can be involved in chemical reactions as intermediates. Spectroscopic identification of the intermediates provides information about their structural as well as dynamical properties, which can be useful in quantifying the fate of different species involved in the chemical reaction. Due to their transient nature and presence of unpaired electrons, obtaining spectroscopic information of these important species has been an experimental as well as a theoretical challenge. The advancement in experimental techniques to isolate these species, laser technology, and quantum chemistry 
calculations have made it possible to obtain spectroscopic identification of transient species in recent times. These reactions affect atmospheric and combustion chemistry, both of which affect almost every aspect of life on earth. Various intermediates, like alkyl alkoxy and peroxy radicals, are involved in these chemical reactions.

\subsection{Alkoxy Radicals}

Alkoxy radicals (RO) are important reaction intermediates in the combustion of fossil fuels and the atmosphere, especially in the troposphere. ${ }^{1-3}$ Spectroscopic study of the free radicals namely substituted and unsaturated alkoxy and peroxy radicals can advance our understanding of many important chemical processes, including combustion of fossil fuels and biofuels in internal combustion engines, and oxidation of hydrocarbons in the atmosphere and interstellar space. ${ }^{3-5}$ The oxidation of hydrocarbon is among the most important chemical processes. In these oxidative processes, alkoxy radicals are often a crucial intermediate that can react further via one of several mechanisms. The chemistry of the alkoxy radicals has been well investigated. ${ }^{6-8}$ Figure $1.1^{8}$ shows the reaction mechanism in hydrocarbon combustion at low-temperature between about 500 and 1000 K. Alkoxy radicals are formed by branching reaction. The oxidation of hydrocarbon is initiated by hydroxyl $(\mathrm{OH})$ radicals and formed peroxy radicals that can react and decompose to produce alkoxy radicals. Also, in the presence of $\mathrm{NO}_{\mathrm{x}}$, alkoxy radicals are formed from alkanes in the troposphere predominantly by the following mechanism:

$$
\mathrm{RO}_{2}+\mathrm{RO}_{2} \rightarrow \mathrm{ROOR}+\mathrm{O}_{2} \rightarrow 2 \mathrm{RO}
$$




$$
\begin{gathered}
\mathrm{ROOH} \rightarrow \mathrm{RO}+\mathrm{OH} \\
\mathrm{RO}_{2}+\mathrm{NO} \rightarrow \mathrm{RO}+\mathrm{NO}_{2}
\end{gathered}
$$

The reactions of the simplest oxygen-containing hydrocarbon, e.g., alkoxy (RO) and peroxy (ROO) radicals are of considerable interest in atmospheric chemistry. ${ }^{1,9}$ Recently open-chain alkoxy radicals- methoxy $\left(\mathrm{CH}_{3} \mathrm{O}\right),{ }^{10-20}$ ethoxy $\left(\mathrm{C}_{2} \mathrm{H}_{5} \mathrm{O}\right),{ }^{10,21-24}$ and isopropoxy $\left(\mathrm{C}_{3} \mathrm{H}_{7} \mathrm{O}\right)^{25-27}$ have been studied extensively, and their spectroscopic information has become available. While the smaller alkoxy radicals have drawn significant attention, the spectroscopic study of larger alkoxy radicals is much sparser. It was generally felt that the fluorescence quantum yield for the $\tilde{B}-\tilde{X}$ transition would rapidly decrease as non-radiative decay processes became dominant in the larger alkoxy radicals. That perception began to change when LIF spectra of iso-butoxy, 2-methyl -1-butoxy, isopentoxy $t$-pentoxy, 3-pentoxy, $t$-butoxy, and 2-butoxy were observed at near ambient temperature. ${ }^{28-31}$

The reactions of alkoxy radicals in the atmosphere are significant and hence of great interest. The most important reactions are decomposition, reaction with $\mathrm{O}_{2}$ and isomerization. ${ }^{1}$ Among many alkoxy radicals we have studied, isobutoxy, 2-methyl -1butoxy, and isopentoxy are especially interesting in dynamics studies because of its $\beta \mathrm{C}-\mathrm{C}$ bond scission. Most alkoxy radicals have multiple structural and conformational isomers. It is well-known that the rate constants of alkoxy reactions can be highly sensitive to their conformations in the ground and excited states. For instance, the energy barrier for the isomerization of ground-state 1-butoxy (a 1,5 $\mathrm{H}$-shift) is $3.6 \mathrm{kcal} / \mathrm{mol}$ lower than decomposition, whereas the barrier of isobutoxy isomerization (a 1,4 H-shift) is 10.6 
$\mathrm{kcal} / \mathrm{mol}$ higher than that of decomposition. ${ }^{29}$ This is due to the fact that the isomerization of isobutoxy occurs via a six-membered transition state, while the transition state of 1butoxy isomerization contains a five-membered ring that has higher strain energy and is less stable. Eventual stable products of particular hydrocarbon oxidation are determined by the competition between various potential reaction pathways of alkoxy radicals.

Cyclic-alkoxy radicals are another important reaction intermediates that still remain unexplored despite those play significance roles in combustion fuels and the atmosphere. For example, the cyclohexoxy radical is a key intermediate in the oxidation of cyclohexane, which is an important constituent of automobile fuel. Among cyclic-alkoxy radicals, the cyclohexoxy radicals and its substituted derivatives like methylcyclohexoxy (MCHOs) are of special interest due to their particular stability of the six-membered ring structure and complicated conformational behavior. ${ }^{32}$ In addition, the cyclohexoxy radical, as well as other cyclic alkoxy radicals, is a prototypical pseudo-Jahn-Teller (pJT) molecule. Analysis of the vibronic structures of $\mathrm{MCHO}$ radicals helps to understand the coupling between close-lying potential energy surfaces (PESs), including pJT and spin-orbit (SO) splitting. ${ }^{24}$, ${ }^{33}$ The lowest three electronic states $\tilde{X}, \tilde{A}$, and $\tilde{B}$ of alkoxy radicals are well defined in the literature. $^{26,32,34,35}$ Its two lowest electronic states can be separated by as low as $61 \mathrm{~cm}^{-1}$. Essentially all alkoxy radicals can be treated as the alkyl substitution of the methoxy radicals $\left(\mathrm{CH}_{3} \mathrm{O}\right)$, which has $C_{3 v}$ symmetry and is orbitally doubly degenerate. In the primary, secondary and tertiary alkoxy radicals such as ethoxy, cyclohexoxy and MCHO, the alkyl substitution reduces the molecular symmetry from $C_{3 v}$ to $C_{s} / C_{1}$ and removes the orbital degeneracy. The $\tilde{X}^{2} \mathrm{E}$ state of the methoxy radical therefore splits into two closelying states ( $\tilde{X}$ and $\tilde{A}$ ) with $\mathrm{A}^{\prime}$ and $\mathrm{A}^{\prime \prime}$ symmetry, which have the half-filled orbital of the 
oxygen-atom within the $C_{s}$ plane $\left(p_{x}\right)$ and perpendicular to it $\left(p_{y}\right)$. the different alkyl groups interact differently with the $p_{x}$ and $p_{y}$ orbitals, which results in different $\tilde{A}-\tilde{X}$ energy separation between the lowest vibrational levels of these two states. The mechanism of interaction between these two close-lying states is of significant theoretical interest. Chemical kinetics and intramolecular dynamics of MCHOs, including alkoxy radicals, in general, are also affected by these lowest-energy electronic states $(\tilde{X}$ and $\tilde{A})$.

Conformation-specific studies of alkoxy radicals, especially that of the correlation between their structures and their reactivities, are, therefore, of significant importance to atmospheric chemistry. The dispersed fluorescence (DF) spectra of alkoxy radicals, which elucidate the energy level structure of lower electronic states of the free radical. It is therefore of interest to obtain the DF spectra of isobutoxy and make a comparison with the other three isomers. The DF spectra of alkoxy radicals can also be used to test the conformational assignment of the laser-induced fluorescence (LIF) spectrum previously reported.

This situation began to change recently with reports of the laser-induced fluorescence (LIF) spectrum of iso-butoxy, 2-methyl -1-butoxy and isopentoxy by Liang et al. ${ }^{29}$ The observed LIF spectra for the electronic transition from $\tilde{B} \leftarrow \tilde{X}$ state corresponds to the promotion of one electron from $\sigma$ orbital of the C-O bond to the half-filled nonbonding orbital of the $\mathrm{O}$ atom. However, they were failure to reported conformational assignments by using their structured LIF spectra of iso-butoxy and 2-methyl-1-butoxy. Furthermore, they did not assign their reported LIF spectrum of isopentoxy radicals. Also, Lin et $a l .{ }^{36}$ and $\mathrm{Wu}$ et al. ${ }^{37}$ reported the first observation of jet-cooled LIF excitation spectra of all four MCHO isomer. Their spectra stimulated us to try to obtain simpler, jet- 
cooled LIF and DF spectra of these species. Such spectra can give us valuable information on the photo-physical and dynamical properties of these radicals.

We have studied the vibrational structure of the isopentoxy by jet-cooled LIF spectroscopy. Also, we have studied the vibrational structures of the isobutoxy, 2-methyl 1-butoxy, isopentoxy, and MCHOs by jet-cooled DF spectroscopy. The recorded spectrum has been analyzed and assigned on the basis of vibrational frequencies and Franck-Condon factors $(\mathrm{FCF})$ predicted by quantum chemical calculations. The intensity pattern and spectral distribution of the vibronic transition in the DF spectra depend on geometry and symmetry of $\tilde{X}, \tilde{A}$, and $\tilde{B}$-states.

\subsection{Peroxy Radicals}

Understanding the mechanism of combustion reactions is critical for advancing internal combustion engine design, improving the efficiency of ignition chemical reactions, and reducing pollutant formation. In order to model the extremely complicated chemical kinetics of combustion processes, ${ }^{3,38}$ it is critical to detect and identify transient reactive chemical intermediates, particularly free radicals. Many intermediates in fossil fuel combustion, however, remain elusive or unidentified. Peroxy radicals (ROO') are the single most important reaction intermediates in low-temperature combustion because they initiate the chain reactions. ${ }^{39,40}$ In low-temperature combustion $(<700 \mathrm{~K})$, the mechanism of peroxy radical formation begins with the abstraction of a hydrogen atom from the hydrocarbon fuel $(\mathrm{RH})$ by a hydroxyl radical $(\mathrm{OH})$ to form an alkyl radical $(\mathrm{R} \cdot)$. The alkyl 
radical undergoes a 3-body reaction with molecular oxygen to form an alkyl peroxy radicals $\left(\mathrm{RO}_{2} \cdot\right)^{9,}{ }^{99-42}$

$$
\begin{gathered}
\mathrm{OH}+\mathrm{RH} \rightarrow \mathrm{H}_{2} \mathrm{O}+\mathrm{R} \\
\mathrm{R}+\mathrm{O}_{2}+\mathrm{M} \rightarrow \mathrm{RO}_{2}+\mathrm{M} \\
\mathrm{RO}_{2} \rightarrow \mathrm{QOOH} \\
\mathrm{QOOH} \rightarrow \mathrm{QO}+\mathrm{OH}
\end{gathered}
$$

Once the peroxy radical is formed, the reaction can branch in several ways, as shown in Figure 1.1. ${ }^{8} \mathrm{HO}_{2}$ can react with it yielding an alkyl hydroperoxide which can decompose into an alkoxy radical and $\mathrm{OH}$, or the peroxy radical can eliminate $\mathrm{HO}_{2}$ directly to give an alkene. The isomerization of peroxy radicals leads to the formation of QOOH species. The rate of QOOH formation is governed by how large the ring structure intermediate and the type of the hydrogen atoms present (primary, secondary, tertiary). The formation of the QOOH species can lead to a chain branching step in which two additional $\mathrm{OH}$ radicals are formed along with an oxy radical.

Eventually, this will lead to an explosive increase in radical concentration, oxidation rate, and temperature to give autoignition, or "engine knocking," which is an uncontrolled burn of the hydrocarbon fuel and the limiting factor in the thermodynamic efficiency of the engine. In order to decrease engine knock the molecular structure of the fuel must be considered with regard to preventing the peroxy radical isomerization to QOOH. ${ }^{43-48}$ When more branched hydrocarbons are used in the fuel, hydrogen atom 
transfer will have to proceed through three and four-membered rings, which have high ring strain and will prevent the formation of QOOH.

Peroxy radical chemistry is also important in the atmospheric oxidation of volatile organic compounds (VOCs), such as alkanes. It is well known that their chemistry plays a key role in the atmosphere for the formation of ozone $\left(\mathrm{O}_{3}\right)$, secondary organic aerosol (SOA), and other secondary pollutants. ${ }^{39,49-51}$ Approximately $1300 \mathrm{Tg}$ of hydrocarbons are emitted each year into the atmosphere from biogenic and anthropogenic sources. ${ }^{52}$ The oxidation of hydrocarbons in the atmosphere are initiated by $\mathrm{OH}$ or $\mathrm{Cl}$, which are also present in the atmosphere and formed alkyl radicals. ${ }^{53}$ The alkyl radical then reacts rapidly with molecular oxygen in the presence of a third body (M, M-typically $\mathrm{N}_{2}$ ), which stabilizes the peroxy radical product by collision. The peroxy radicals are involved in an important reaction cycle of ozone production in the atmosphere, particularly in the troposphere. For example, $\mathrm{O}_{3}$ can be produced in the troposphere, where it is considered a pollutant, via peroxy radicals' reaction with NO:

$$
\begin{gathered}
\mathrm{NO}+\mathrm{RO}_{2} \rightarrow \mathrm{NO}_{2}+\mathrm{RO} \\
\geq 430 \mathrm{~nm} \\
\mathrm{NO}_{2} \rightarrow \mathrm{NO}+\mathrm{O} \\
\mathrm{O}\left({ }^{3} \mathrm{P}\right)+\mathrm{O}_{2}+\mathrm{M} \rightarrow \mathrm{O}_{3}+\mathrm{M}
\end{gathered}
$$

Peroxy radicals reaction with $\mathrm{NO}$ produces excess amounts of $\mathrm{NO}_{2}$ in the troposphere, where it can be photolyzed by sunlight to generate oxygen atoms. The oxygen atoms then react with molecular oxygen to produce tropospheric $\mathrm{O}_{3}{ }^{41,54}$ which, is 
considered a health hazard. The second reaction of peroxy radicals with NO in the troposphere can produce excess amounts of hydroxyl radical:

$$
\begin{gathered}
\mathrm{NO}+\mathrm{RO}_{2} \rightarrow \mathrm{NO}_{2}+\mathrm{RO} \\
\mathrm{RO}+\mathrm{O}_{2} \rightarrow \mathrm{R}^{\prime} \mathrm{C}=\mathrm{O}+\mathrm{HO}_{2} \\
\mathrm{HO}_{2}+\mathrm{NO} \rightarrow \mathrm{NO}_{2}+\mathrm{OH} \\
\mathrm{OH}+\mathrm{O}_{3} \rightarrow \mathrm{HO}_{2}+\mathrm{O}_{2}
\end{gathered}
$$

The $\mathrm{OH}$ radicals can then migrate from the troposphere to the stratosphere, where $\mathrm{O}_{3}$ is considered a filter against UV radiation, and reduce the amount of $\mathrm{O}_{3}$ present putting our health at risk..$^{55}$

In addition to the atmospheric oxidation of alkanes by $\mathrm{OH}$ and $\mathrm{Cl}$ radicals, large quantities of alkenes emitted into the atmosphere are readily oxidized by them as well and undergo reactions that can pollute the troposphere. ${ }^{54,56-58}$ The double bond of an alkene can be attacked by $\mathrm{OH}$ to give a hydroxy alkyl radical that can react with molecular oxygen in a three-body reaction to yield hydroxyperoxy radicals. ${ }^{59-65}$ Allyl group-containing peroxy radicals are particularly important because they are generated in significant quantities by the $\mathrm{OH}$-initiated oxidation of isoprene, the most reactive and most abundant non-methane biogenic hydrocarbon. ${ }^{66-69}$ It has been shown in Figure $1.2^{72}$ that there are four of the six possible isomeric peroxies formed in the early stages of isoprene oxidation. $^{70-72}$

The atmospheric oxidation of methyl allyl can also serve as a prototype for larger alkenes, such as isoprene and other unsaturated hydrocarbons, which are emitted in even 
higher quantities. After methane (emission rates of $\sim 600 \mathrm{Tg} / \mathrm{yr}$ ), ${ }^{52}$ isoprene is the most abundant hydrocarbon emitted into the atmosphere with global emission rates reaching between 450-500 Tg/yr and an atmospheric concentration between 1-30 ppb. ${ }^{52,73,74}$ As with other alkenes isoprene has a very high reactivity towards $\mathrm{OH}$ radical which adds across a double bond followed by reaction of the resulting radical with molecular oxygen. This is combined with the high concentration of isoprene in the atmosphere makes the $\mathrm{OH} / \mathrm{O}_{2}+$ isoprene reaction a critical atmospheric process, and it has been the subject of many theoretical and experimental studies. ${ }^{60-62,65,70,75-81}$

For many years the best way to spectroscopically observe peroxy radicals was to probe their $\tilde{B} \leftarrow \tilde{X}$ absorption located in the UV region. However, there is a considerable disadvantage to using this transition to distinguish among different peroxy radicals. The $\tilde{B}$ $\leftarrow \tilde{X}$ transition generally involves the promotion of an electron from the second-highest occupied molecular orbital (HOMO-1) to the singly occupied molecular orbital (SOMO). Hence, the $\tilde{B} \leftarrow \tilde{X}$ transition involves the promotion of an electron from a bonding orbital to an antibonding orbital so that when a peroxy radical undergoes a transition to the state, the $\mathrm{O}-\mathrm{O}$ bond dissociates ${ }^{82}$ (Figure 1.3). This gives rise to a repulsive $\tilde{B}$-state potential energy surface, and the resulting spectrum for all peroxy radicals is a broad featureless absorption near $240 \mathrm{~nm}$. This lack of any observable structure makes the UV transition a poor diagnostic with which to identify different peroxy radicals. Alternatively, the $\tilde{A} \leftarrow \tilde{X}$ transition of peroxy radicals is located in the near-IR and is a HOMO to SOMO transition. The HOMO of peroxy radicals is nonbonding along the OO bond $\tilde{A} \leftarrow \tilde{X}$ making the transition a promotion of an electron from a nonbonding to antibonding orbital. Therefore, the $\tilde{A}$-state is a bound state, and upon excitation, to the $\tilde{A}$-state, there is only a lengthening 
of the O-O bond instead of complete dissociation. These qualities indicate that the $\tilde{A} \leftarrow \tilde{X}$ spectra of peroxy radicals will be structured and characteristic of a given $\mathrm{RO}_{2}$ radical. This allows the differentiation among different peroxy radicals and makes the $\tilde{A} \leftarrow \tilde{X}$ transition an excellent diagnostic with which to observe peroxy radicals. However, observing the $\tilde{A}$ $\leftarrow \tilde{X}$ transition may be experimentally difficult as it is based on the highly forbidden $\mathrm{a}^{1} \Delta_{g}-\tilde{X}^{3} \Sigma_{g}{ }^{-}$transition of the $\mathrm{O}_{2}$ chromophore. ${ }^{83}$ Consequently, the cross section for this transition is $\approx 10^{4}$ times smaller than the $\tilde{B} \leftarrow \tilde{X}$ transition. Unfortunately, traditional absorption experiments do not have the sensitivity to detect this weak transition. However, the CRDS technique can be used to overcome the obstacle of studying a trace gas species with a weak transition.

Laser spectroscopy is a powerful tool for identification and diagnostics of chemical reaction intermediates; hence, it is of great importance to energy research. Different experimental methods were used for the efficient detection of the alkoxy and peroxy intermediates. LIF and DF techniques were used to identify different electronic states. LIF and DF are a sensitive background free technique for probing fluorescing species. DF spectra for the $\tilde{B} \rightarrow \tilde{X}$ and $\tilde{B} \rightarrow \tilde{A}$ transitions of isobutoxy and 2-methyl-1-butoxy are presented in Chapter (IV). LIF and DF spectra for the $\tilde{B} \leftarrow \tilde{X}, \tilde{B} \rightarrow \tilde{X}$, and $\tilde{B} \rightarrow \tilde{A}$ transitions of isopentoxy is presented in the Chapter (V). In Chapters VI and VII, DF spectra for the $\tilde{B} \rightarrow \tilde{X}$ and $\tilde{B} \rightarrow \tilde{A}$ transitions of 1-, 2-, 3-, and 4-MCHO are discussed. Quantum chemical calculations have been carried out to assist the identification of the spectral carriers and simulation of experimentally obtained spectra, from which molecular parameters such as electronic transition energies and vibrational frequencies of both electronic states can be determined. These parameters, in return, can be used to benchmark the calculations. 
Notwithstanding the achievements, the capability of the current LIF/DF apparatus is limited by the nature of the spectroscopic techniques and the laser sources available. In order to gain a better understanding of the free radicals currently being studied in our lab and to include more combustion intermediates into future spectroscopic investigations, these limitations need to be overcome by employing new spectroscopic techniques and radiation sources. Cavity ring-down spectroscopy (CRDS), being an absorption technique with high sensitivity, is an ideal option. Because most molecules have absorptions due to electronic transitions in the UV/visible region, even when the excited state is radiationless, the absorption-based CRDS technique is more versatile than LIF/DF technique. Near-IR CRDS spectrum for the $\tilde{A}-\tilde{X}$ transition methyl allyl peroxy radical is presented in Chapter VIII. 


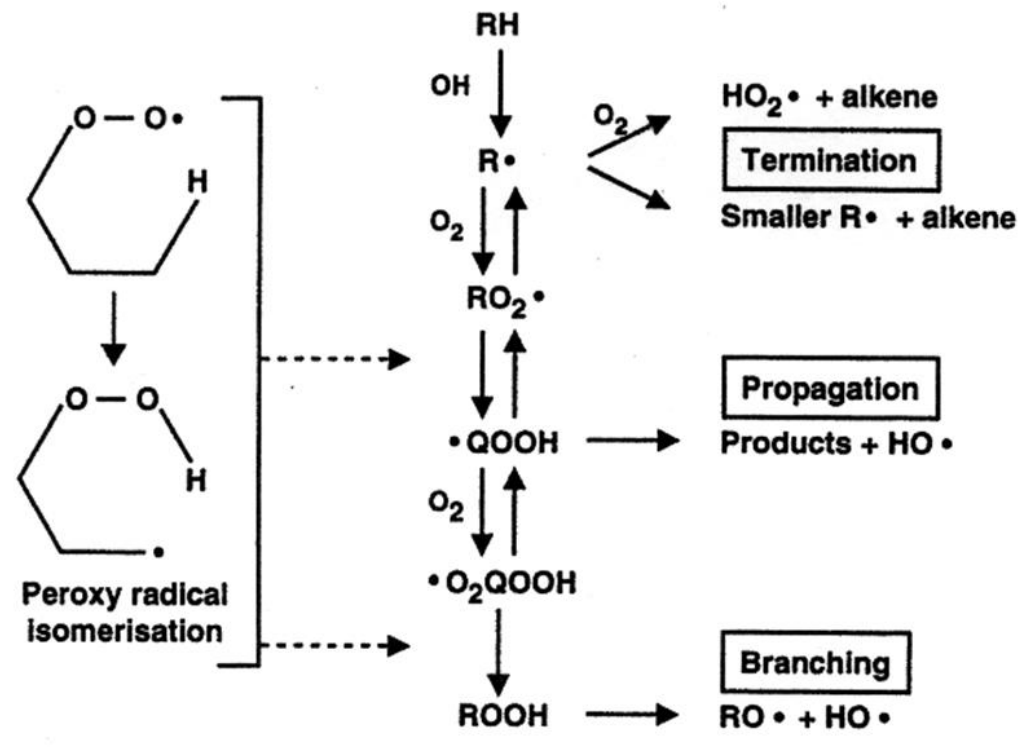

Figure 1.1. Schematic diagram for combustion of hydrocarbons taken from ref. [8]. 


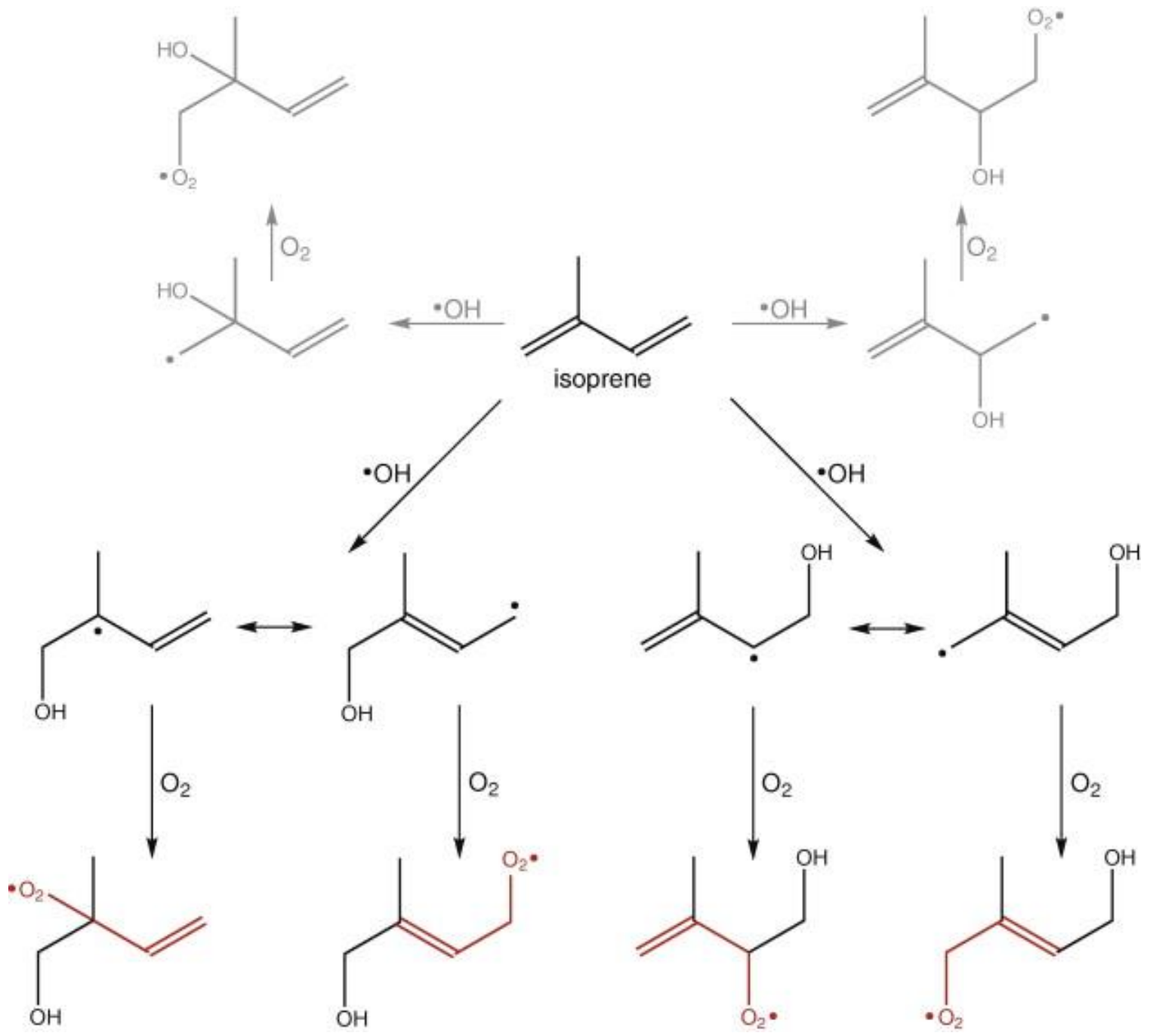

Fig. 1.2. Mechanism of peroxy radical formation from $\mathrm{OH}$-initiated oxidation of isoprene. The allyl peroxy functionality (highlighted in red) is achieved in four of the six possible addition sequences taken from ref. [72]. 


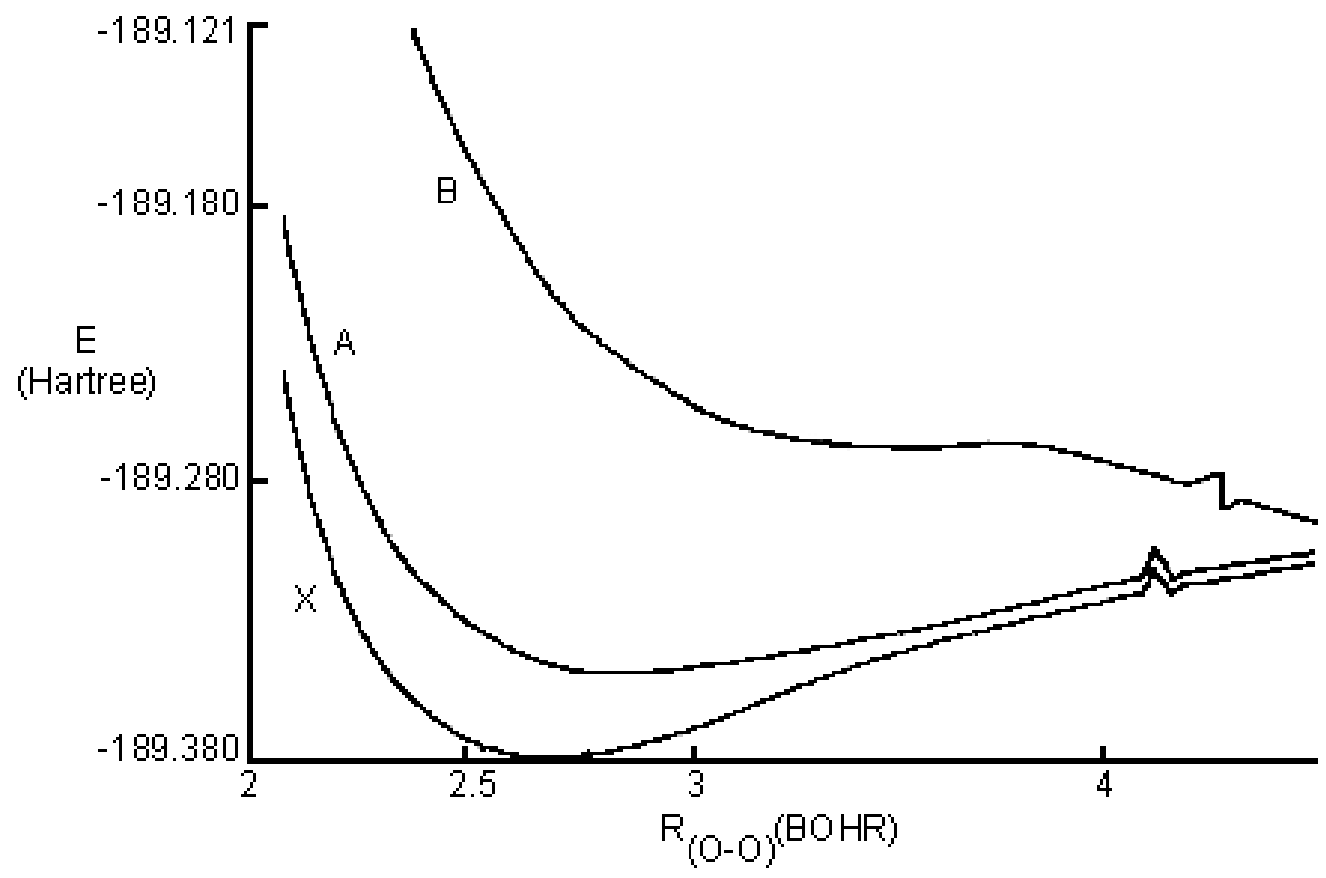

Figure 1.3. Methyl peroxy potential energy surfaces of the $\tilde{X}, \tilde{A}$ and $\tilde{B}$-states as a function of Roo taken from ref. [83]. 


\section{CHAPTER II}

\section{EXPERIMENTAL METHODS}

\subsection{Laser-Induced Fluorescence (LIF) and Dispersed Fluorescence (DF)}

Spectroscopy

Laser-induced fluorescence (LIF) and Dispersed Fluorescence (DF) technique coupled with supersonic jet expansion have been used to study the $\tilde{B} \leftrightarrow \tilde{X}$ transition of various primary, ${ }^{15,28,84,85}$ secondary, ${ }^{28,84}$ tertiary, ${ }^{84}$ and unsaturated ${ }^{86,87}$ alkoxy radicals. Moderateresolution, as well as high-resolution spectra, have been obtained and analyzed to give accurate transition energies, vibrational frequencies, and rotational/spin-rotational constants. $^{88,}{ }^{89}$ It was therefore reasonable to try to obtain similar LIF, and DF characterization of isobutoxy, 2-methyl-1-butoxy, isopentoxy and methyl substituted cyclohexoxy radicals.

\subsubsection{Introduction}

LIF and DF spectroscopy was used to obtain spectra of fluorescing radicals and stable species. In LIF spectroscopy, fluorescence emitted by a sample is collected as a function of the probing laser wavelength. Depending on the electronic structure of a sample, it can absorb laser radiation at specific wavelengths. Following absorption, the newly populated 
excited states can decay back to the ground state through fluorescence. Fluorescence from the sample is collected through a lens system perpendicular to the probing radiation and focused on a photomultiplier (PMT) tube. The collection of fluorescence perpendicular to the direction of the laser radiation minimizes the background signal detected by the PMT from the scattered probing radiation. LIF spectroscopy provides high sensitivity due to its background free nature.

\subsubsection{Moderate Resolution LIF Experimental Setup}

Figure 2.1 shows the schematic diagram for the LIF setup used for the alkoxy radicals experiment. This setup is similar to the one described previously. ${ }^{15,28,84,90-92}$ The alkyl nitrite was stored in a stainless-steel reservoir and expanded into a vacuum chamber through a $0.5 \mathrm{~mm}$ pinhole nozzle with $200 \mathrm{psi}$ of backing pressure of helium gas to create a supersonic jet showing in figure 2.2. A $355 \mathrm{~nm}$ photolysis beam generated by the third harmonic of an Nd:YAG laser (Continuum, Powerlite Precision II 8000) was focused at the throat of the expansion to photolyze the O-NO bond of the nitrite precursor near the orifice of the pulsed solenoid nozzle (General Valve, series 9). The free radicals produced were excited to the $\tilde{B}$ electronic state by a frequency-doubled pulsed dye laser (Sirah, Cobra-Stretch) pumped by the second harmonic $(532 \mathrm{~nm})$ of an Nd:YAG laser (SpectraPhysics, GCR-4) at about a $10 \mathrm{~mm}$ downstream distance. The UV probe laser beam was generated by an Nd:YAG (532 nm) pumped dye laser with pyridine 1, pyridine 2, Styryl 8, and DCM (Exciton) laser dyes. The dye laser beam was frequency-doubled (by a KDP crystal)) to provide a tunable output to scan in the $27000-32200 \mathrm{~cm}^{-1}$ frequency range. For the LIF measurement, the fluorescence was focused onto a photomultiplier tube (PMT, 
Hamamatsu, H10721-01). The PMT output was integrated by a boxcar averager (Stanford Research System, SR250) and sent to a PC via an analog-to-digital converter card (AdLink, PCI-9812). All the spectra were calibrated using a wavemeter (HighFinesse, WS-7). A relative frequency error of $<1.00 \mathrm{~cm}^{-1}$ is expected in the low-resolution survey scans, mainly due to uncertainty in the peak position of different vibrational bands.

\subsubsection{Moderate Resolution DF Experimental Setup}

In figure 2.1 shows the schematic diagram for the DF setup used for the isobutoxy, 2methyl-1-butoxy, isopentoxy, and methyl substituted cyclohexoxy radicals experiment. The experimental setup used to obtain DF spectra has also been discussed in detail elsewhere. ${ }^{34,93-99}$. For the DF measurement, the fluorescence was focused on and dispersed by a monochromator (Acton Research, SpectraPro 300i) with an 1800 grooves/mm grating with $300 \mu \mathrm{m}$ entrance size slit and then imaged onto an intensified charge-coupled device (CCD, Princeton Instruments, PI-MAX 512). The image was collected using the WinSpec software. The spectral resolution of the DF spectra is $\sim 30 \mathrm{~cm}^{-1}$.

\subsubsection{Radical Production Chemistry}

The alkoxy radicals were produced by the photolysis of corresponding alkyl nitrites (RONO) using the third harmonic of an Nd:YAG laser at $355 \mathrm{~nm}$.

$$
\mathrm{RONO}+\mathrm{h} v \rightarrow \mathrm{RO}+\mathrm{NO}
$$


Isobutyl nitrite was purchased from Sigma-Aldrich and used without further purification, while 2- methyl-1-butyl nitrite, isopentyle nitrite, and methyl cyclohexyl nitrites were synthesized by following well-established alkyl nitrite synthesis procedure described by Blatt: 100

$$
\mathrm{ROH}+\mathrm{HONO} \underset{\sim 0^{\circ} \mathrm{C}}{\stackrel{\mathrm{H}_{2} \mathrm{SO}_{4}}{\longrightarrow}} \mathrm{RONO}+\mathrm{H}_{2} \mathrm{O}
$$

Briefly, the alkyl nitrite precursors were synthesized by the addition of sulfuric acid and the appropriate alcohol to an aqueous solution of sodium nitrite. $25.1 \mathrm{ml}$ of concentrated ( 97\%) sulfuric acid was mixed with $100 \mathrm{ml}$ of water. $0.75 \mathrm{~mol}$ of appropriate alcohol (1,2,3,4-methylcyclohexanol, isopentanol, or 2-methyl-1-butanol) was added to the sulfuric acid solution. Protons from the acidic solution can form hydrogen bonds with the hydroxy group of the alcohol and make the hydroxy group a better leaving group (Eqn. 2.3).

$$
\mathrm{ROH}+\mathrm{H}^{+} \rightarrow \mathrm{ROHH}^{+}
$$

The sulfuric acid and alcohol mixture was added dropwise to $100 \mathrm{ml}$ of $0.7 \mathrm{M}$ sodium nitrite solution kept at $0^{\circ} \mathrm{C}$ over a period of about 120 minutes. The nitrite group then removes the hydroxy group from the alcohol in a substitution reaction to give the alkyl nitrite precursor (Eqn. 2.4).

$$
\mathrm{ROHH}^{+}+\mathrm{NO}_{2}^{-} \rightarrow \mathrm{RONO}+\mathrm{H}_{2} \mathrm{O}
$$


Alkyl nitrite was then separated from the aqueous layer using a separatory funnel. All the chemicals for the synthesis were purchased from Sigma-Aldrich. There are minor impurities, mainly solvent, but they were not expected to dissociate with the $355 \mathrm{~nm}$ photolysis laser. All observed LIF signals are dependent on the photolysis laser.

\subsection{Cavity Ring-Down Spectroscopy (CRDS)}

Alkyl peroxy radicals possess a strong $\tilde{B}-\tilde{X}$ electronic transition in the $\mathrm{UV}$, which has been used to follow them in various kinetics experiments. However, the $\tilde{B}$-state is dissociative, leading to a broad and structure-less spectrum, as is characteristic of all peroxy radicals, making the UV transition a poor diagnostic to selectively identify different peroxies or to use to characterize the geometric and electronic structure of the molecule. ${ }^{82,101}$ Unlike the $\tilde{B}$-state, the $\tilde{A}$-state is bound. Thus, the $\tilde{A}-\tilde{X}$ transition yields spectra with a well-defined structure allowing one to distinguish between different peroxy radicals as well as different isomers and conformers of a given peroxy radical. Miller and coworkers have identified several alkyl peroxy radicals via their $\tilde{A}-\tilde{X}$ electronic transitions in the near-infrared (NIR) using CRDS. ${ }^{102}$ Moderate to high-resolution NIR-CRDS experiments have been performed for several peroxy radicals to give accurate transition energies, vibrational frequencies, and rotational constants. ${ }^{102-104}$ Thus, the CRDS technique was used to obtain the spectrum for the $\tilde{A}-\tilde{X}$ transition of methyl allyl peroxy results of which are presented in Chapter VII of this dissertation. 


\subsubsection{Introduction}

Cavity ring-down spectroscopy (CRDS) is a simple, highly sensitive direct absorption technique based on the rate of absorption of light circulating in an optical cavity. CRDS can be used to study atoms and molecules in the gas and condensed phase and is especially powerful for measuring strong absorptions of species present in trace amounts or weak absorptions of abundant species. The transmitted intensity follows the Beer-Lambert Law

$$
\mathrm{I}=\mathrm{I}_{0} \cdot \exp (-\sigma \mathrm{NL})
$$

where $\mathrm{I}_{0}$ and $\mathrm{I}$ are the incidents and transmitted light measured before and after an absorbing species, $\sigma$ is the absorption cross section of the molecule at a given wavelength, $\mathrm{N}$ is the number density of the absorber per unit volume, and $\mathrm{L}$ is the path length of light through the absorber. The sensitivity of an experiment depends on the $\mathrm{I}_{0}$, and $\mathrm{I}$ that means how well $\mathrm{I}$ and $\mathrm{I}_{0}$ can be differentiated from each other. In a traditional single pass absorption experiment, I and $\mathrm{I}_{0}$ are monitored as a function of wavelength. If one is attempting to detect a species that absorbs very weakly and is present in small concentrations, then traditional absorption experiments will not be successful because the difference between $\mathrm{I}$ and $\mathrm{I}_{0}$ is undetectable. Hence, most absorption methods lack the sensitivity needed to investigate weak absorption cross-sections and for low concentrations of the absorbing molecule. Cavity ringdown spectroscopy (CRDS) is a modification of a traditional absorption experiment that utilizes a multipass absorption technique to increase the path length along a physically small dimension effectively.

In CRDS, light is coupled into a high finesse optical cavity made up of two highly reflective mirrors, as shown in Figure 2.3. The fraction of light that is trapped within the 
cavity is reflected back and forth between the two mirrors. At each reflection, a small portion of light leaks out of the cavity and is detected. A very small portion of the trapped photons leaks through each of the mirrors on each round trip. The light leaking out of the cavity decreases exponentially with consecutive round trips, and the intensity of the radiation leaked out is measured as a function of time and recorded. The decay curve of the intensity as a function of time is fit to an exponential function, yielding the ring-down time. Beer-Lambert law can be adapted to calculate the fractional loss of the intensity of light in a cavity.

$$
I_{t}=I_{0} \cdot \exp \left[-\left(\frac{\text { loss }}{\text { reflection }}\right)\left(\frac{\text { number of reflections }}{\text { round trip }}\right)(\text { number of round trips })\right]
$$

The number of reflections per round trip is 2 , because of two mirrors in a cavity. The number of round trips ( $\mathrm{n}$ ) in time $\mathrm{t}$ would then be given by

$$
\mathrm{n}=\frac{\mathrm{tc}}{2 \mathrm{~L}}
$$

Where $\mathrm{L}$ is the length of the ring down cavity, and $\mathrm{c}$ is the speed of light. If $\mathrm{R}$ is taken as the reflectivity of the mirrors, then the loss per reflection would be (1-R). Eq. (2.7) can then be modified as

$$
\mathrm{I}_{\mathrm{t}}=\mathrm{I}_{0} \cdot \exp \left[-(1-\mathrm{R})\left(\frac{\mathrm{tc}}{\mathrm{L}}\right)\right]
$$

The intensity of light $\left(\mathrm{I}_{\mathrm{t}}\right)$ then decays with a time constant, viz, the empty-cavity ring-down time, $\tau_{0}$, which is defined as the time for the intensity to decay to the $1 / \mathrm{e}$ of the initial intensity $\left(\mathrm{I}_{0}\right)$. In an empty cavity, $\tau_{0}$ is given by 


$$
\tau_{0}=\frac{\mathrm{L} / \mathrm{c}}{(1-\mathrm{R})}
$$

When an absorbing sample is introduced inside the cavity, the loss of intensity of light due to absorption is given by

absorption loss $=($ loss per round trip $)($ number of round trip $)=(2 \sigma N 1) \frac{\text { tc }}{2 \mathrm{~L}}$

Where 1 is the length of the absorbing sample inside the cavity which could be smaller or equal to the length of the cavity L. The total loss in the intensity of light is then given by

$$
\text { total loss }=(1-\mathrm{R})\left(\frac{\mathrm{tc}}{\mathrm{L}}\right)+(\sigma \mathrm{Nl})\left(\frac{\mathrm{tc}}{\mathrm{L}}\right)=[(1-\mathrm{R})+(\sigma \mathrm{Nl})] \frac{\mathrm{tc}}{\mathrm{L}}
$$

Beer-Lambert law for a rind-down cavity with an absorber is therefore:

$$
\mathrm{I}_{\mathrm{t}}=\mathrm{I}_{0} \cdot \exp \left\{-[(1-\mathrm{R})+\sigma \mathrm{Nl}]\left(\frac{\mathrm{tc}}{\mathrm{L}}\right)\right\}
$$

The ring-down time of the cavity with the absorbing sample, $\tau_{\mathrm{abs}}$, is given by

$$
\tau_{\mathrm{abs}}=\frac{\mathrm{L} / \mathrm{c}}{(1-\mathrm{R})+\sigma \mathrm{Nl}}
$$

Eqn. (2.9) and (2.13) can be then be combined to give

$$
\sigma \mathrm{Nl}=\frac{\mathrm{L}}{\mathrm{c}}\left(\frac{1}{\tau_{\mathrm{abs}}}-\frac{1}{\tau_{0}}\right)
$$

There are two main benefits of CRDS when compared with typical absorption spectroscopy experiments. The first is the greatly increased effective path length and hence the extremely high sensitivity. For a typical flow-cell CRDS experimental setup, $\mathrm{L}=\mathrm{l}=0.5$ $\mathrm{m}, \mathrm{R}=99.995 \%$, the empty-cavity ring-down time is $33 \mu \mathrm{s}$, and the effective path length is 
$10 \mathrm{~km}$. Thanks to the long path length, a minimum detectable absorption coefficient of $\sim 0.3$ ppm per pass can be achieved.

The second significant advantage of CRDS is the insensitivity to fluctuations in incident radiation intensity. Unlike in traditional multipass absorption spectroscopy, in which intensity of the transmission is used to determine the absorbance, in CRDS, it is determined from change of the decay constant when the absorbing sample is introduced into the cavity.

\subsubsection{Room Temperature Experimental Setup}

The apparatus for the room temperature experiment is represented in Figure 2.4. The experimental setup used to obtain CRD spectra has also been discussed in detail elsewhere. ${ }^{105-108}$ The required NIR radiation, around $1.3 \mu \mathrm{m}$, was generated by a Raman shifting of the output of a dye laser in molecular hydrogen. The second harmonic of Nd:YAG (PRO- 270, Spectra-Physics) is pumping a Sirah dye laser system with a $20 \mathrm{~Hz}$ repetition rate. Operated with DCM laser dye (Exciton), the system produced 90-130 $\mathrm{mJ} /$ pulse of tunable radiation in the region of $605-640 \mathrm{~nm}$ with a linewidth of $0.6 \mathrm{~cm}^{-1}$. Initial frequency calibration was performed with Ocean Optics Wave meter and was typically within a $0.25 \mathrm{~cm}^{-1}$ from the dye laser dial reading. Dye laser resolution is limited by the resolution of the dye laser grating. The output of the dye laser was focused by a lens ( $\mathrm{f}=50 \mathrm{~cm}$ ) into a $93 \mathrm{~cm}$ long single Raman cell filled with 200-220 psi of $\mathrm{H}_{2}$. The output radiation from the Raman cell was spectrally filtered using several $1.0 \mu$ m cut-off longwave filters (Corion LL-1000-F) to eliminate anti-stokes and $1^{\text {st }}$ stokes radiation. The laser beam 
is collimated by a telescope consisting of two confocal lenses $(\mathrm{f}=7.5$ and $2.5 \mathrm{~cm})$ and before being injected into the ring-down cavity. A $100 \mu \mathrm{m}$ pinhole is placed at the focal point of the telescope for mode selection. The resulting 1-2 $\mathrm{mJ}$ of the second stokes radiation in the $1.25-1.35 \mu \mathrm{m}$ region was delivered to the ringdown cell through two $50 \mathrm{~cm}$ focal length lenses. The ringdown cavity was fabricated of stainless steel and consisted of a $20 \mathrm{~cm}$ long central part with two rectangular UV grade quartz photolysis windows and two $15 \mathrm{~cm}$ long arms. The cavity ringdown mirrors (purchased from Los Gatos Research Inc.) were positioned at the ends of the arms on adjustable mounts.

An ArF and KrF excimer laser (Lambda-Physik, LPX 120i) were used to provide the $193 \mathrm{~nm}$ and $248 \mathrm{~nm}$ photons for photolysis. A set of diverging and converging cylindrical lenses was used to reshape the photolysis laser beam cross section to a $12 \times 0.5$ $\mathrm{cm}^{2}$ rectangle. The fluence of the photolysis beam was measured to be $\sim 50 \mathrm{~mJ} / \mathrm{cm}^{2}$. A set of diverging and converging cylindrical lenses was used to reshape the photolysis laser beam cross section to a $12 \times 0.5 \mathrm{~cm}^{2}$ rectangle. The fluence of the photolysis beam was measured to be $\sim 50 \mathrm{~mJ} / \mathrm{cm}^{2}$. CRD mirrors (Los Gatos Research, $\mathrm{R}>99.99 \%$ ) were mounted on both ends of the reaction cell to form a ring-down cavity with a length of $L \sim 55 \mathrm{~cm}$. The photolysis laser was sent through the photolysis quartz windows into the central part of the flow cell, thereby determining the volume wherein radicals are created. Under this condition, all infrared photons will interact with produced radicals, and radicals will be distributed uniformly within the probe beam.

The outlet of the flow cell was connected through the needle valve to the vacuum pump. The following procedure was used to determine the pumping speed. Pure nitrogen and oxygen were introduced into the cell through a calibrated mass flow controller (MKS), 
and the pressure in the cell was measured against several readings of the flow controller. The voltage from each mass-flow controller was sent to a digital display box. The slope of the obtained pressure vs. gas flow dependence will represent pumping speed. $\mathrm{N}_{2}$ gas flow carried vapor of methyl-substituted allyl chloride precursors into the reaction cell. Saturation vapor pressure of the precursors was controlled and stabilized by immersing precursor reservoirs in a 1:1 water-ethylene glycol bath (about $-10^{\circ} \mathrm{C}$ ). Gas flow rates were as follows: precursor in $\mathrm{N}_{2}: 100-200 \mathrm{sccm} ; \mathrm{O}_{2}: 500-750 \mathrm{sccm}$. The partial pressure of the $\mathrm{N}_{2}$ gas flow for purging the CRD mirrors and the UV side windows for the photolysis beam was controlled by a needle valve to $\sim 50$ Torr, while the total pressure in the reaction cell was $\sim 150$ Torr. The frequency of the dye laser was measured by a wavelength meter (HighFinesse, WS-7, absolute accuracy=60 MHz). The linewidth of the near-IR radiation is estimated to be about $1 \mathrm{GHz} .{ }^{109,110}$ A Thorlabs InGaAs (CS10) photodiode was used to detect the outcoming radiation in the $1.2-1.7 \mu \mathrm{m}$ region.

Transmission of the near-IR radiation through the ring-down cavity was focused on and detected with an amplified InGaAs photodiode (Thorlabs, PDA10CS) and recorded with an oscilloscope (Tektronix, DPO3014). The empty-cavity ring-down time was $~ 150$ $\mu$ s. Averaged ring-down decay curves were sent to a PC and fit by a home-built LabVIEW program. Ring-down decay curves were collected at each wavelength with the excimer photolysis laser on and off. Absorption (in ppm/pass) recorded with the photolysis laser off, i.e., absorption due to precursors was subtracted from that with the photolysis laser on to obtain net absorption due to products. The time delay between the photolysis and the CRD laser pulses can be varied for kinetic measurements. Lifetimes of MAOO peroxy radicals were measured to be $\sim 1 \mathrm{~ms}$. 


\subsubsection{Peroxy Radical Production}

Methyl allyl peroxy radicals were produced in a room-temperature stainless steel gas-flow reaction cell by $193 \mathrm{~nm}$ or $248 \mathrm{~nm}$ photolysis of corresponding methyl-substituted allyl chlorides in the presence of excessive $\mathrm{O}_{2}$. Three commercially available precursors were used: 3-chloro-2-methyl-1-propene (97\%, TCI), 3-chloro-1-butene (97\%, Sigma-Aldrich), and 75\% 1-chloro-2-butene and 25\% 3-chloro-1-butene mixture (TCI). Production of methylallyl (MA) radicals by photolysis (Reaction 1) is followed by oxygen addition (Reaction 2) to form the peroxy radicals R00 ', where R' is the MA radical:

$$
\begin{array}{ll}
\mathrm{R}-\mathrm{Cl} \stackrel{\mathrm{h} \nu(\lambda=193 \mathrm{~nm})}{\longrightarrow} \mathrm{R} \cdot+\mathrm{Cl} \\
\mathrm{R}^{\cdot}+\mathrm{O}_{2} \longrightarrow \mathrm{ROO} .
\end{array}
$$

The MAOO radical has three isomers (1-, 2-, and 3-MAOO's). $193 \mathrm{~nm}$ photolysis of 3chloro-2-methyl-1-propene produces the 2-MA radical (Figure 2.5a). It is a resonance hybrid with two contributing structures: $\mathrm{CH}_{2}=\mathrm{C}\left(\mathrm{CH}_{3}\right) \mathrm{CH}_{2}$ and $\mathrm{CH}_{2} \mathrm{C}\left(\mathrm{CH}_{3}\right)=\mathrm{CH}_{2}$. Oxygen addition to either structure forms the 2-MAOO radical.

When 1-chloro-2-butene or 3-chloro-1-butene is used as a precursor, the direct photolysis products are 1-MA radicals with the unpaired electron at the primary $\left(\mathrm{CH}_{3} \mathrm{CH}=\mathrm{CHCH}_{2}{ }^{\circ}\right)$ and secondary $\left(\mathrm{CH}_{3} \mathrm{CH} \cdot \mathrm{CH}=\mathrm{CH}_{2}\right)$ positions, respectively (Figures $2.5 \mathrm{~b}$ and 2.5c). These are two contributing structures of a resonance-stabilized free radical. The nascent radicals produced by photolysis interconvert via allylic rearrangement:

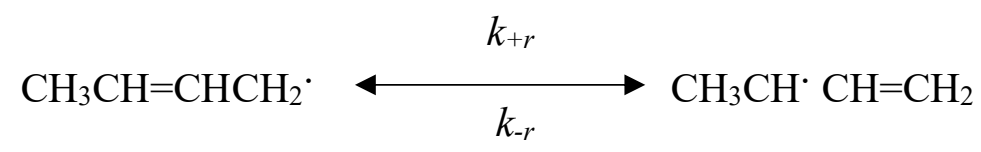


Oxygen additions to both nascent MA radicals produced by photolysis and secondary free radicals via allylic rearrangement are possible (Figures $2.5 \mathrm{~b}$ and $2.5 \mathrm{c}$ ). Terminal addition produces 1-MAOO:

$$
\mathrm{CH}_{3} \mathrm{CH}=\mathrm{CHCH}_{2} \cdot+\mathrm{O}_{2} \stackrel{k_{1-M A O O}}{\longrightarrow} \mathrm{CH}_{3} \mathrm{CH}=\mathrm{CHCH}_{2} \mathrm{OO} .
$$

while non-terminal addition produces 3-MAOO:

$$
\mathrm{CH}_{3} \mathrm{CH} \cdot \mathrm{CH}=\mathrm{CH}_{2}+\mathrm{O}_{2} \stackrel{k_{3-M A O O}}{\longrightarrow} \mathrm{CH}_{3} \mathrm{CHOO} \cdot \mathrm{CH}=\mathrm{CH}_{2}
$$

The population ratio of 1- and 3- MAOO radicals produced is therefore determined by the reaction rate constants $\left(k_{ \pm \mathrm{r}}, k_{1-\mathrm{MAOO}}, k_{3-\mathrm{MAOO}}\right)$ of Reactions $2.17-2.19$. Note that we also used $248 \mathrm{~nm}$ (KrF excimer) photolysis laser, but no CRD signal of MAOO was observed. All peaks observed in the CRD spectra that are assigned to MAOO radicals depend on the presence of the stable precursors and oxygen, $193 \mathrm{~nm}$ photolysis laser pulses, and delay time between the photolysis and CRD laser pulses shorter than $\sim 3 \mathrm{~ms}$. 


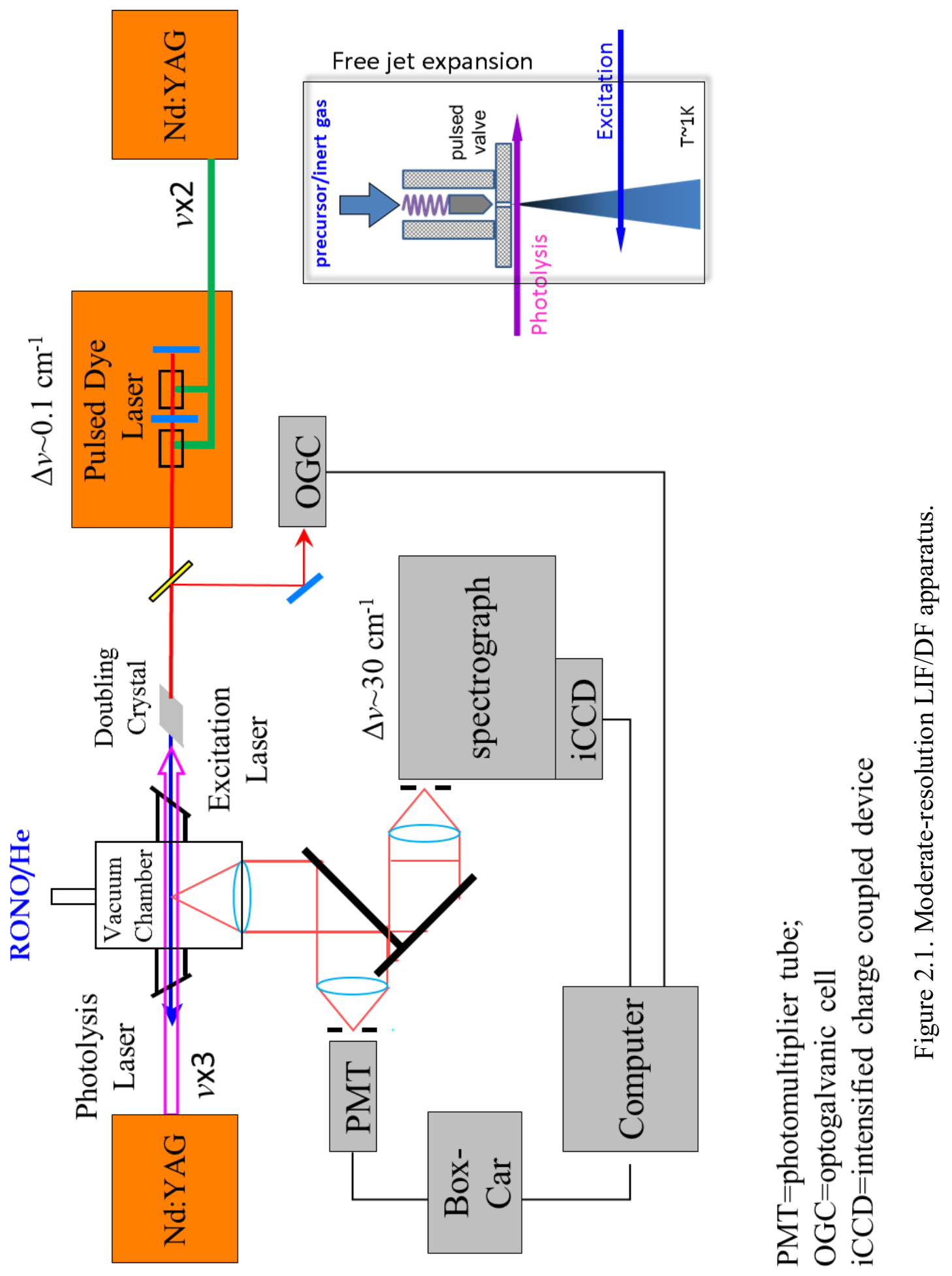




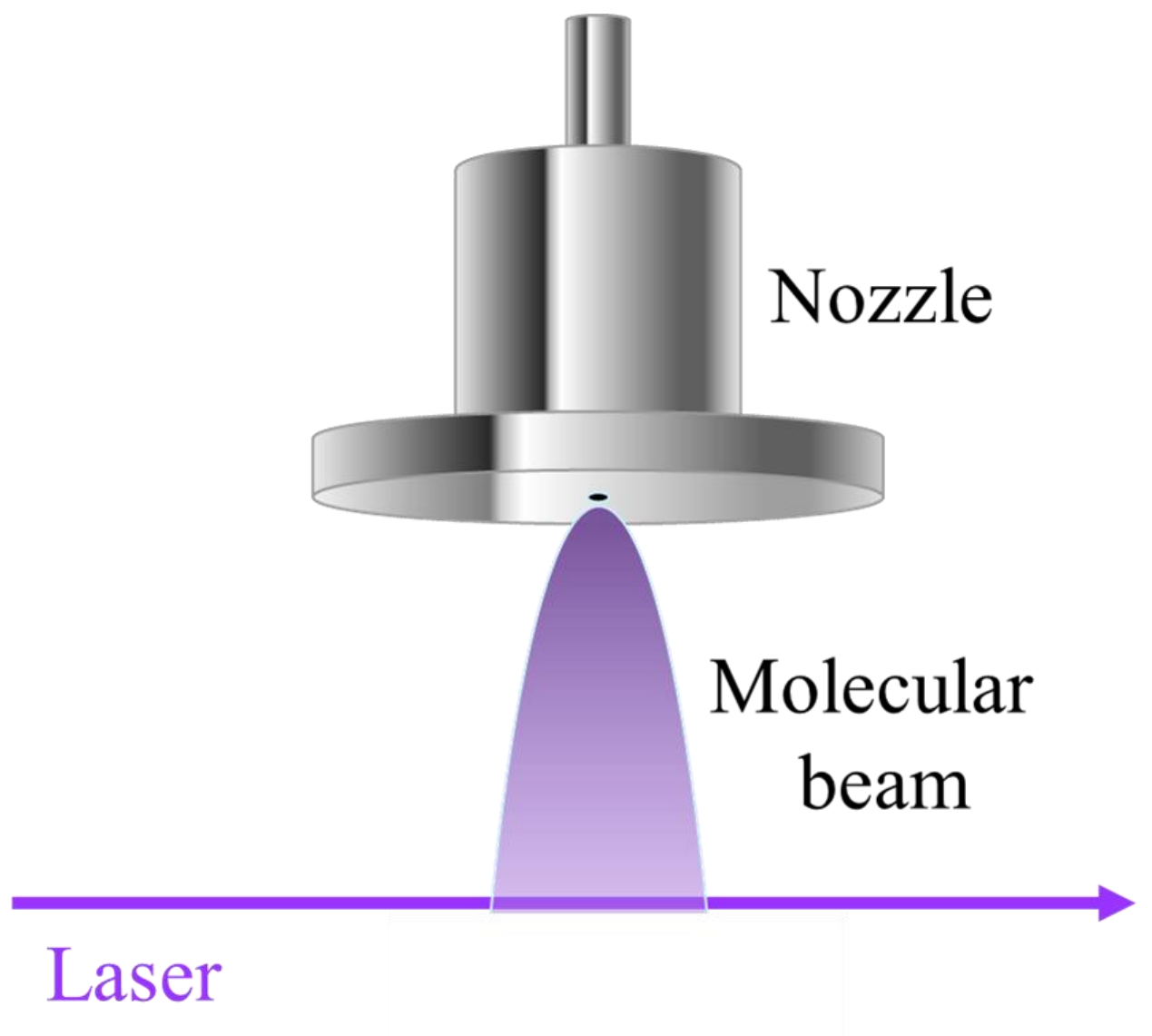

Figure 2.2. Supersonic cooling 


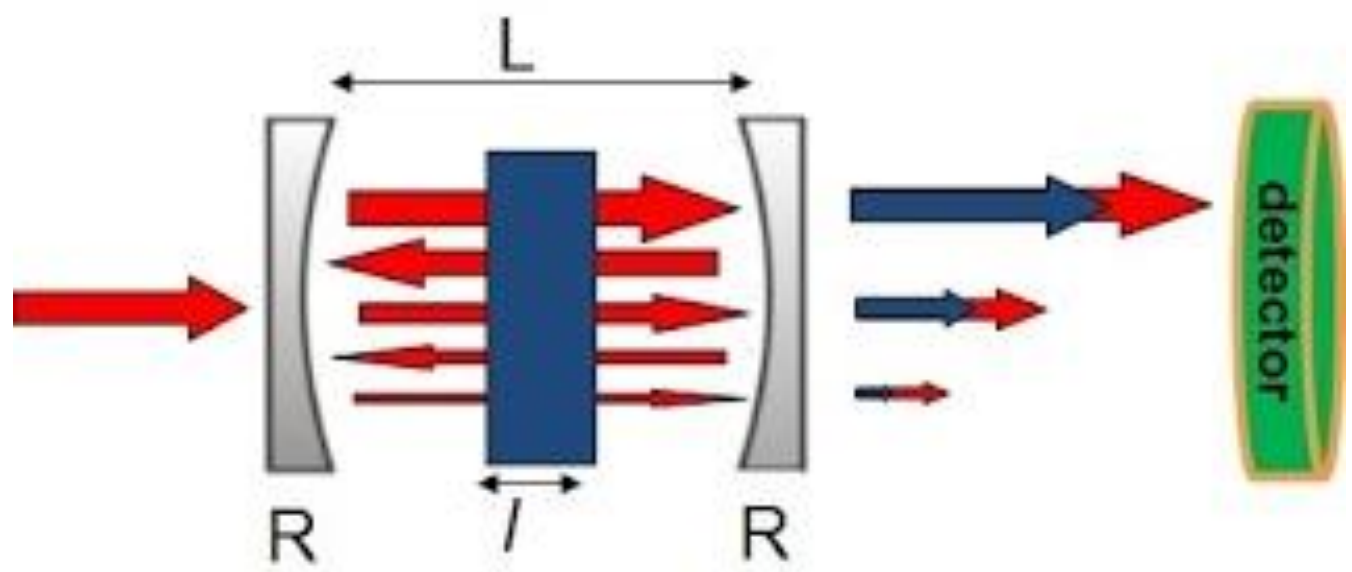

Figure 2.3. Principle of Cavity ring-down spectroscopy. 


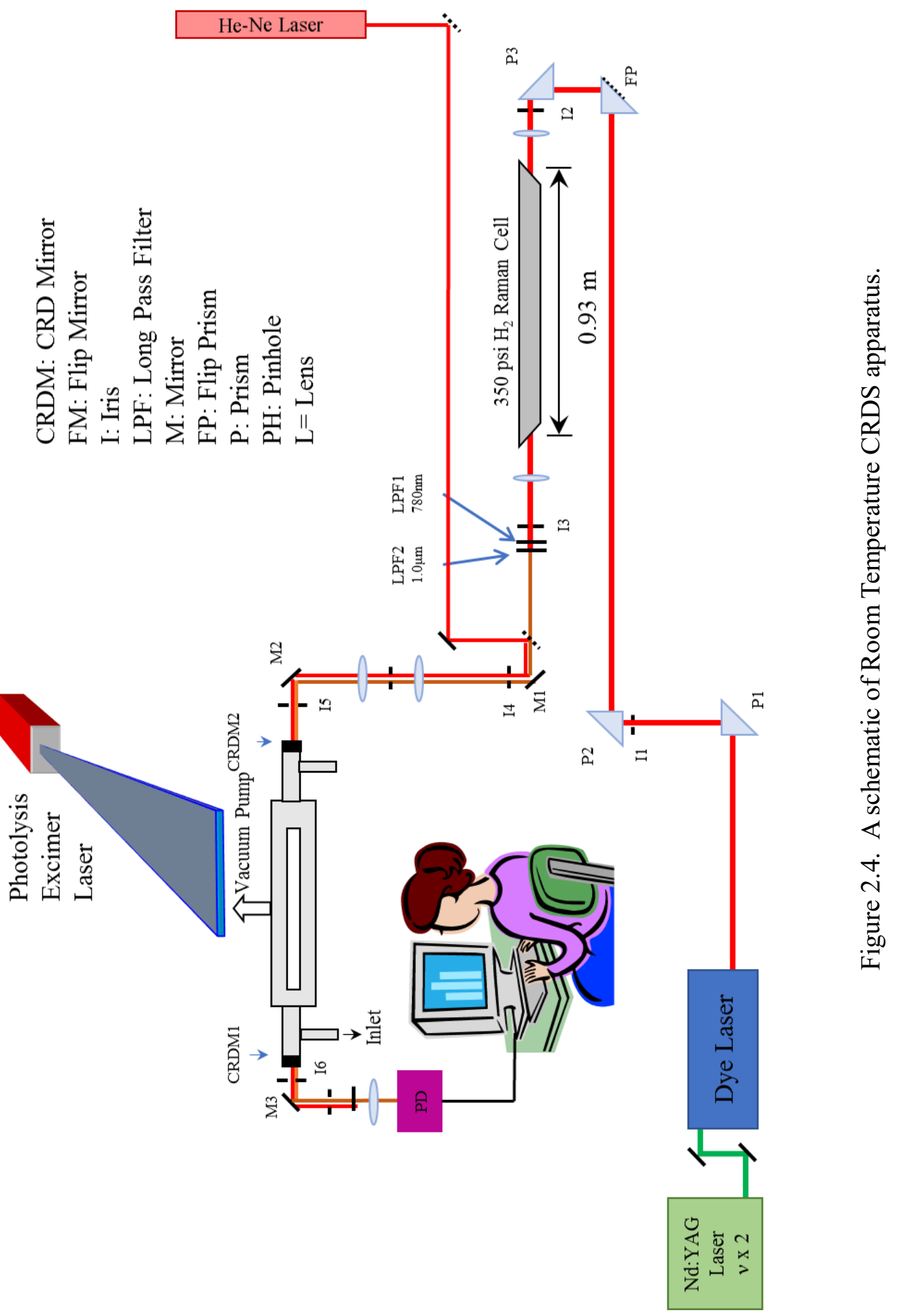




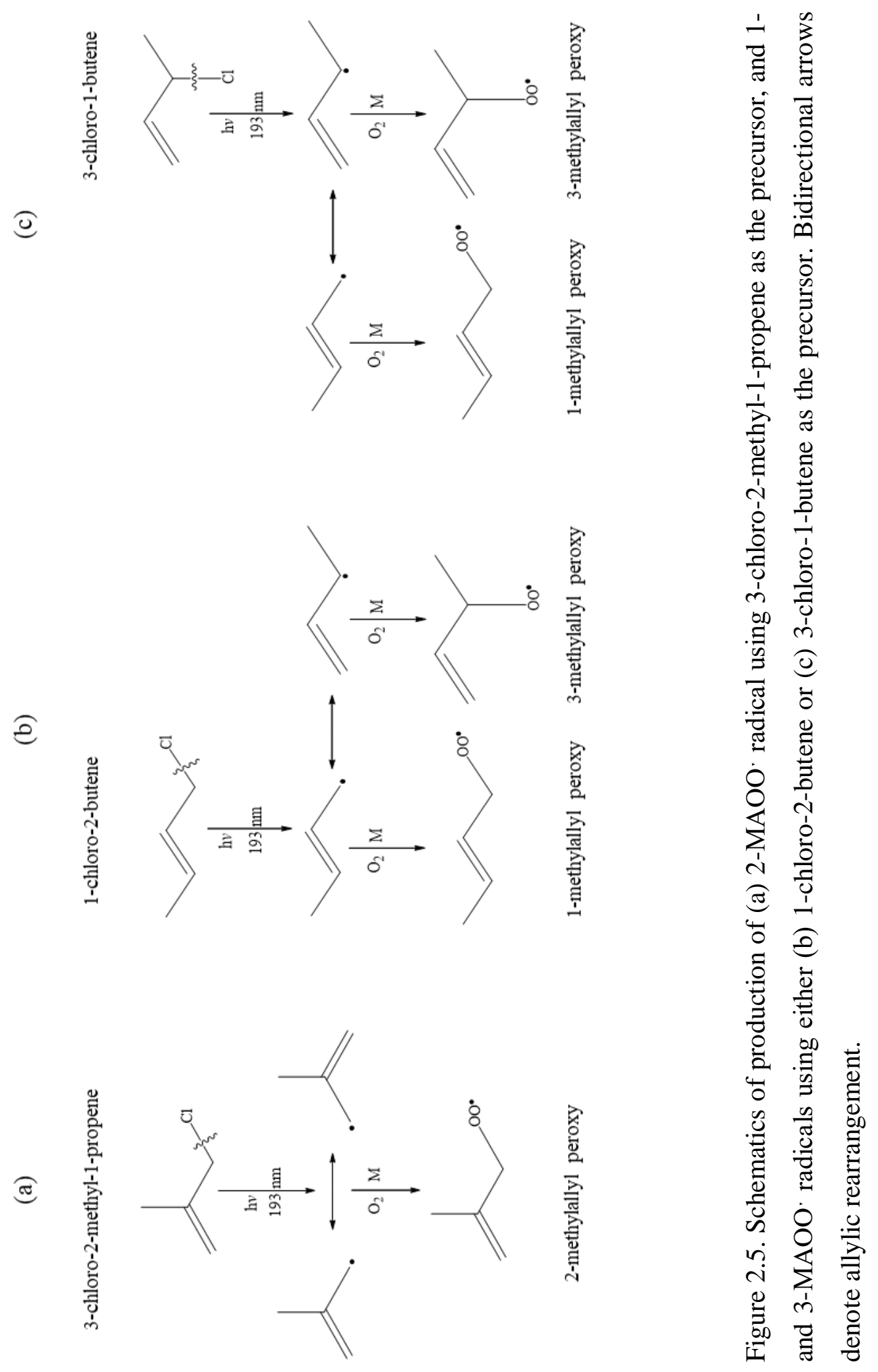




\section{CHAPTER III}

\section{THEORY OF SPECTRAL SIMULATION}

\subsection{Introduction}

Electronic spectra of polyatomic molecules are more complicated than those of the diatomic molecules for various reasons. First, the number of vibrational modes in a polyatomic molecule is $3 N-6(3 N-5$ in a linear molecule), where $N$ is the number of atoms in the molecule. Therefore, the larger the number of atoms in the molecule, the larger the number of vibrational modes, and hence the more congested the electronic spectrum is due to more vibronic (vibrational-electronic) transitions. The spectral congestion is further exacerbated by more complicated molecular geometry and often lower molecular symmetry, which introduces more allowed vibronic transitions. Moreover, polyatomic molecules usually have three rotational constants, whereas diatomic molecules have only one rotational constant. (For diatomic molecules, the two rotational constants for rotations around the two principal axes perpendicular to the internuclear axis are identical). Usually, polyatomic molecules have larger moment of inertia and, hence, smaller rotational constants than diatomic molecules. As a result, the rotational levels of medium to large-sized polyatomic molecules are so close to each other that the rotational and fine structure of their vibronic transitions cannot be resolved 
using nanosecond pulsed lasers (typical linewidth $\sim 0.1 \mathrm{~cm}^{-1}$ ). Finally, the spectra of polyatomic molecules can be complicated by the existence of multiple conformations, as we shall see in this Dissertation. It is, therefore important to review the basic theory and methodology for prediction and simulation of vibronic spectra of polyatomic molecules. In such spectral prediction and simulation, both the transition frequencies (i.e., transition energies) and transition intensities need to be calculated, which we will discuss in the rest of this chapter.

\subsection{Energy Level Structure of Polyatomic Molecules}

\subsubsection{Born-Oppenheimer (BO) Approximation}

The Born-Oppenheimer Principle (or Born-Oppenheimer Approximation) states that the electrons in a molecule move so much faster than the nuclei that the two kinds of motion are independent: the electrons see the nuclei as being stationary, and so each electron doesn't have to adjust its motion to maintain a minimized electron-nucleus interaction energy. Thus, we can calculate the purely electronic energy of a molecule at different molecular geometries, then the internuclear repulsion energy, and add the vibrational energies of nuclei to get the total molecular energy. If we assume the nuclei and electrons in a molecule to be point masses and neglect spin-orbit and other relativistic corrections, then the molecular Hamiltonian can be written compactly by this equation

$$
\hat{H}=\hat{T}_{n}+\hat{T}_{e}+\hat{V}_{n n}+\hat{V}_{e n}+\hat{V}_{e e}
$$

The first two terms in Eq. (3.1) are the kinetic energy operators of the nuclei $\left(T_{\mathrm{n}}\right)$ and electrons $\left(T_{\mathrm{e}}\right)$, respectively, the third term is the potential energy of repulsion between the 
nuclei $\left(V_{\mathrm{nn}}\right)$, the fourth term is the potential energy of attraction between the electrons and the nuclei $\left(V_{\text {en }}\right)$, and the fifth term is the potential energy of repulsion between the electrons $\left(V_{\mathrm{ee}}\right)$

The nuclear kinetic energy $\left(T_{\mathrm{n}}\right)$ can be separated from the total molecular Hamiltonian, the rest terms of the molecular Hamiltonian (Eq. 3.1) can be written as $H_{\mathrm{el}}+V_{\mathrm{nn}}$, where $H_{\mathrm{el}}$ $=T_{\mathrm{e}}+V_{\mathrm{en}}+V_{\mathrm{ee}}$ is the pure electronic Hamiltonian. The Schrodinger equation with $H_{\mathrm{el}}+V_{\mathrm{nn}}$ is

$$
\left(H_{\mathrm{el}}+V_{\mathrm{nn}}\right) \Psi_{\mathrm{el}}=U \Psi_{\mathrm{el}}
$$

In Eq. (3.2), $U$ is the electronic energy, including internuclear repulsion. Under the BornOppenheimer Approximation, $\Psi_{\mathrm{el}}$ is a function of only electronic coordinates. Therefore, the contribution of $V_{\mathrm{nn}}$ to molecular energy is a constant shift of all energy levels at each molecular geometry. One can, therefore, further separate $V_{\mathrm{nn}}$ from the total molecular Hamiltonian. Eq. (3.3) reduces to:

$$
H_{\mathrm{el}} \Psi_{\mathrm{el}}=E_{\mathrm{el}} \Psi_{\mathrm{el}}
$$

where $E_{\mathrm{el}}$ is the pure electronic energy of the molecule. $E_{\mathrm{el}}$ and $\Psi_{\mathrm{el}}$ can be solved $a b$ initio, followed by calculation of the vibrational energy level structure of each electronic state at fixed nuclear coordinates.

\subsection{2 ab initio Computations}

Numerous calculations have been done to predict and simulate LIF and DF spectra of alkoxy radicals and CRDS spectra of peroxy radicals. These computational data include i) the $\tilde{B}-\tilde{X}$ adiabatic excitation energies of alkoxy radicals and the $\tilde{A}-\tilde{X}$ adiabatic excitation 
energies of peroxy radicals, ii) vibrational modes and frequencies in all aforementioned electronic states, especially the $\mathrm{C}-\mathrm{O}$ stretch of alkoxy radicals and the $\mathrm{O}-\mathrm{O}$ stretch of the peroxy radicals; and iii) relative energies of different isomers and conformers of each investigated molecule in their ground states. Density functional theory (DFT) method using the B3LYP, ${ }^{111}$ or CAM-B3LYP ${ }^{112}$ functionals has been used for the ground electronic $(\tilde{X})$ state calculations, while the $\tilde{B}$ state of alkoxy radicals and the $\tilde{A}$ state of peroxy radicals were calculated using the configuration interaction single (CIS) ${ }^{113}$ and time depended (TD)DFT methods. For molecules with the $C_{s}$ symmetry, the first excited state (the ground state of its own symmetry) can be calculated using the DFT method with the HOMO and LUMO orbitals of the unpaired electron swapped. (See Section 4.2) For low-lying electronic states, the adiabatic excitation energy calculated using the DFT method is significantly more accurate than those calculated using the CIS or TD-DFT method.

All calculations have been performed by the Gaussian09 program, ${ }^{114}$ using standard basis sets, including 6-31+G (d), 6-311+G(d,p).

\subsubsection{Molecular Vibrations}

In the research reported in the present dissertation, harmonic frequencies of vibrational modes of involved electronic states are calculated $a b$ initio. Energies of vibrational levels of each electronic state with respect to the minimum of the potential energy surface (PES) is, therefore:

$$
E_{v}=\sum_{i=1}^{3 N-5 \text { or } 3 N-6}\left(v_{i}+\frac{1}{2}\right) h v_{i}
$$


where $v_{\mathrm{i}}$ and $v_{\mathrm{i}}$ are the vibrational quantum number and harmonic vibrational energy of the $i^{\text {th }}$ vibrational mode, respectively. Usually, vibrational energies are given in the form of the wavenumber and in the unit of $\mathrm{cm}^{-1}$. In this case, $h v$ is replaced by the harmonic frequency $\omega_{e}$, and the wavenumber of a vibrational level with respect to the minimum of the electronic state is:

$$
G_{v}=\sum_{i=1}^{3 N-5 \text { or } 3 N-6}\left(v_{i}+\frac{1}{2}\right) \omega_{e, i}
$$

In real molecules, the vibrational energy level structure is affected by the vibrational anharmonicity. With anharmonicity, high-order terms are included in Eq. (3.5):

$$
G_{v}=\sum_{i=1}^{3 N-5 \text { or } 3 N-6}\left[\left(v_{i}+\frac{1}{2}\right) \omega_{e, i}-\left(v_{i}+\frac{1}{2}\right)^{2} \omega_{e, i} x_{e, i}+\left(v_{i}+\frac{1}{2}\right)^{3} \omega_{e, i} y_{e, i}+\ldots\right]
$$

Usually, we truncate the equation above after the first two terms in the squared brackets, i.e., only the harmonic term and the first-order anharmonic correction $\left(x_{\mathrm{e}} \omega_{e}\right)$ are taken into account. The energy separation between the $v$ and the $v+1$ levels is, therefore:

$$
\Delta G_{v+1 / 2}=G_{v+1}-G_{v}=\omega_{e}-2 \omega_{e} x_{e}
$$

The discrete values $\Delta G_{v+1 / 2}$ as a function of $v_{\mathrm{i}}$ define a straight line (eq 3.7) with a y-axis intercept of $\omega_{e}$ and a slope of $-2 \omega_{e} x_{\mathrm{e}}$. This plot is called the Birge-Sponer plot. From fitting the Birge-Sponer plot of experimental data, the harmonic frequency $\omega_{e}$ and the anharmonicity parameter $x_{\mathrm{e}}$ can be determined. ${ }^{115,116}$

To calculate the vibrational frequencies of a molecule, first, we need to find its equilibrium geometry (within the Born-Oppenheimer approximation). Second, the potential energy 
surface (PES) is expanded in the Taylor series. The zeroth-order is defined as zero. The first order is 0 because of the minimum energy condition. The calculation of second derivatives of the potential energy surface leads to the molecular Hessian, which enables the prediction of vibrational frequencies. ${ }^{117}$

Note that every vibrational mode contributes to the zero-point energy (ZPE):

$$
E_{0}=\sum_{i=1}^{3 N-5 \text { or } 3 N-6} \frac{1}{2} h v_{i}
$$

The ZPEs offset the $v=0$ levels of electronic states from the minima of their PESs and have to be taken into account in calculating the adiabatic electronic transition frequencies $\left(v^{\prime}=0\right.$ $\left.\leftarrow v^{\prime \prime}=0\right)$

\subsection{Transition Intensity}

The transition intensity between two energy levels is proportional to the line strength $(S)$, as well as the lower- and upper-state populations. For an excitation, i.e., an upward transition:

$$
I \propto S\left(N^{\prime \prime}-N^{\prime}\right)
$$

where $N^{\prime}$ and $N^{\prime \prime}$ are the lower and upper state populations, respectively. For electronic transitions, $N^{\prime \prime}>>N^{\prime}$. The equation above can be written as:

$$
I \propto S N^{\prime \prime}
$$

The transition intensity is also proportional to the intensity of the excitation laser, if the transition is not saturated. In our experiments, we use a constant excitation laser intensity 
whenever possible. Otherwise, the experimental spectra are normalized by the laser intensity.

\subsubsection{Separation of the electric transition dipole moment and the Franck-Condon Factor}

The line strength $(S)$ can be written by the following equation:

$$
\mathrm{S} \propto\left(\int \Psi^{\prime}|\hat{\mu}| \Psi^{\prime \prime} \mathrm{d} \tau\right)^{2}
$$

where $\Psi^{\prime \prime}$ and $\Psi^{\prime}$ are overall wave functions of the lower and upper states, respectively. They are products of the electronic, vibrational, and spin wave functions. (Rotational and nuclear spin wavefunctions are neglected in the present research.) $\Psi=\Psi_{\mathrm{e}} \Psi_{\mathrm{v}} \Psi_{\mathrm{s} .} \hat{\mu}$ is the molecular dipole moment (operator), consisting of the electronic and the nuclear components, i.e., $\hat{\mu}=\boldsymbol{\mu}_{\mathbf{e}}+\boldsymbol{\mu}_{\mathbf{N}}$. under the Born-Oppenheimer Approximation, we can separate the electronic and the nuclear wavefunctions. The spin wavefunctions are also treated separately. Upon the separation of the electronic, vibrational, and spin wavefunctions, Eq. (3.11) becomes

$$
\mathrm{S} \propto\left(\int \psi_{\mathrm{e}}^{\prime} \psi_{\mathrm{v}}^{\prime} \psi_{\mathrm{s}}^{\prime}\left|\left(\boldsymbol{\mu}_{\mathrm{e}}+\boldsymbol{\mu}_{\mathrm{N}}\right)\right| \psi_{\mathrm{e}}^{\prime \prime} \psi_{\mathrm{v}}^{\prime \prime} \psi_{\mathrm{s}}^{\prime \prime} \mathrm{d} \tau\right)^{2}
$$

$S \propto\left(\int \psi_{\mathrm{v}}^{\prime} \psi_{\mathrm{v}}^{\prime \prime} \mathrm{d} \tau \int \psi_{\mathrm{e}}^{\prime}\left|\boldsymbol{\mu}_{\mathrm{e}}\right| \psi_{\mathrm{e}}^{\prime \prime} \mathrm{d} \tau \int \psi_{\mathrm{s}}^{\prime} \psi_{\mathrm{s}}^{\prime \prime} \mathrm{d} \tau+\int \psi_{\mathrm{e}}^{\prime} \psi_{\mathrm{e}}^{\prime \prime} \mathrm{d} \tau \int \psi_{\mathrm{v}}^{\prime}\left|\boldsymbol{\mu}_{\mathrm{N}}\right| \psi_{\mathrm{v}}^{\prime \prime} \mathrm{d} \tau \int \psi_{\mathrm{s}}^{\prime} \psi_{\mathrm{s}}^{\prime \prime} \mathrm{d} \tau\right)^{2}$ 
The second term in the parentheses in Eq. 3.13 vanishes under the Born-Oppenheimer Approximation because electronic wavefunctions of different electronic states are eigenfunctions of the pure electronic Hamiltonian $H_{\mathrm{el}}$ and, hence, orthogonal to each other. The remaining (first) term

$$
\mathrm{S} \propto\left(\int \psi_{\mathrm{v}}^{\prime} \psi_{\mathrm{v}}^{\prime \prime} \mathrm{d} \tau \int \psi_{\mathrm{e}}^{\prime}\left|\mu_{\mathrm{e}}\right| \psi_{\mathrm{e}}^{\prime \prime} \mathrm{d} \tau \int \psi_{\mathrm{s}}^{\prime} \psi_{\mathrm{s}}^{\prime \prime} \mathrm{d} \tau\right)^{2}
$$

is the product of three integrals. The first integral $\left(\int{\psi^{\prime}}_{\vee} \psi^{\prime \prime}{ }_{v} d \tau\right)$ is the overlap integral between the vibrational wavefunctions of the two states, and its square is called the FranckCondon factor. The second integral $\left(\int \psi_{\mathrm{e}} \mathrm{e}\left|\boldsymbol{\mu}_{\mathrm{e}}\right| \psi^{\prime \prime}{ }_{\mathrm{e}} \mathrm{d} \tau\right)$ is the electric transition dipole moment between the upper and lower electronic states. The third integral $\left(\int \psi^{\prime}{ }_{s} \psi_{s}{ }_{s} d \tau\right)$ involves the electron spin and leads to the spin selection rule of $\Delta S=0$, where $S$ is the electron spin.

\subsubsection{Franck-Condon factor}

To calculate the intensities of transitions between different vibrational states of two

electronic states, the Franck-Condon (FC) principle may be applied. ${ }^{118,}{ }^{119}$ When a molecule undergoes an electronic transition, the nuclear configuration of the molecule experiences no significant change because that nuclei are much more massive than electrons, and the electronic transition takes place faster than the nuclei can respond. The Franck-Condon principle is, therefore, also called the vertical transition principle. 
Due to the Franck-Condon principle. vibrational bands in an electronic transition vary in intensity, in proportional to the square of the first integral $\left(\int \psi^{\prime}{ }_{\mathrm{v}} \psi^{\prime \prime}{ }_{\mathrm{v}} \mathrm{d} \tau\right)^{2}$ in equation (3.14), which is called the Franck-Condon factor (FCF) for the transition. Figure $3.1^{120}$ shows the vibrational wave functions for some vibrational levels of two electronic states of a diatomic molecule that have different values of $R_{\mathrm{e}}$, the internuclear distance at the equilibrium. Consider a transition from the $v^{\prime \prime}=0$ vibrational level of the ground electronic state to a vibrational level of the excited electronic state. From the figure, we see that $\Psi{ }^{\prime \prime}$ for $v^{\prime \prime}=0$ and $\Psi_{\mathrm{v}}^{\prime}$ for $v^{\prime}=2$ are both large at the same value of $R$; hence these two functions have optimal overlap, and the $v=2 \leftarrow 0$ vibrational band of the electronic transition should be the most intense band (in the absorption or excitation spectrum). Similarly, emission from the $v^{\prime}=2$ level of the excited electronic state gives an intense band for the transition to the $v^{\prime \prime}=0$ level.

\subsubsection{Computation of Franck-Condon factors and the Duschinsky Mixing}

In the research reported in the present dissertation, FCFs were calculated using the ezSpectrum program. ${ }^{121}$ Due to the large difference in $\tilde{X}$-, $\tilde{A}$ - and $\tilde{B}$-state geometries, Duschinsky rotations were taken into account in the calculation ofFCFs to better reproduce the experimental spectra, as explained below.

The normal coordinates of different electronic states of a molecule are usually different due to different molecular geometries and force constants. Thus, electronic transitions can cause mixing of normal modes, i.e., a specific normal mode of the excited electronic state is a linear combination of two or more normal modes of the ground 
electronic state and vice versa. In the multi-dimensional space comprising of the normal modes, this mixing is presented as a rotation of the normal coordinates. This phenomenon was first considered by Duschinsky ${ }^{122}$ when extending the FC principle from diatomic to polyatomic molecules. Duschinsky first proposed that the two sets of normal coordinates are related to each other by a unitary transformation and a displacement vector. (See figure 3.2). The vector gives the displacement between the equilibrium structures of the two states, and the unitary transformation matrix can be viewed as an overlap matrix between the normal modes of the two electronic states. ${ }^{123}$ The closer the squares of the diagonal elements are to unity, the more the normal modes are like each other in form and energy. Large off-diagonal elements indicate a change in the energy ordering or mixing between different normal modes. This mixing is the mathematical expression of a Duschinsky rotation.

\subsubsection{Boltzmann Distribution}

The intensities of lines in a vibronic spectrum also depend on the population of the lower state. For room-temperature experiments, the Boltzmann distribution is used to predict the relative populations of different molecules, different isomers, and conformers of a certain molecule, and different vibrational levels of a certain molecular species. The expression for such calculation is:

$$
\frac{n_{i}}{n_{0}}=\frac{g_{i}}{g_{0}} e^{-E_{i} / k T}
$$

where $n_{0}$ and $n_{\mathrm{i}}$ are populations of the ground and the $i^{\text {th }}$ excited state, respectively. $g_{i}$ and $g_{0}$ are degrees of degeneracy of the two states. $E_{i}$ is the relative energy of the $i^{\text {th }}$ excited 
state (with respect to the ground state). When multiple molecular species (including conformers and isomers) co-exist, $n_{0}$ refers to the ground state of the lowest-energy species, while $E_{i}$ is relative to this lowest state.

We can write the Boltzmann distribution as

$$
p_{i}=\frac{g_{i} e^{-E_{i} / k T}}{q}
$$

where $p_{i}$ is the molar fraction of a molecule in state $i, p_{i}=\mathrm{n}_{\mathrm{i}} / \mathrm{n}_{\text {total }}$, where $\mathrm{n}_{\text {total }}$ is the total population of all molecular species (in all states). $q$ is the molecular partition function:

$$
q=\sum_{i} g_{i} e^{-\frac{E_{i}}{k T}}
$$

It is important to note that the application of the Boltzmann distribution assumes that molecular species are at thermal equilibrium. In our experiments, free radicals are produced from stable molecules. In the room-temperature CRD experiment (see Section 2.2.2), thermal equilibrium is a fairly good approximation because of the relatively high temperature and high pressure. Nascent free radicals reach the thermal equilibrium via collision with buffer gas molecules $\left(\mathrm{N}_{2}\right)$. In the jet-cooled LIF/DF experiments, free radicals may be "trapped" in local maxima of the PES due to insufficient collisions with the carrier gas molecules (He or Ar) before entering the collision-free zone, where they are interrogated by the laser beam. Boltzmann distribution is, therefore, not a good approximation for predicting relative populations in jet-cooled experiments. 


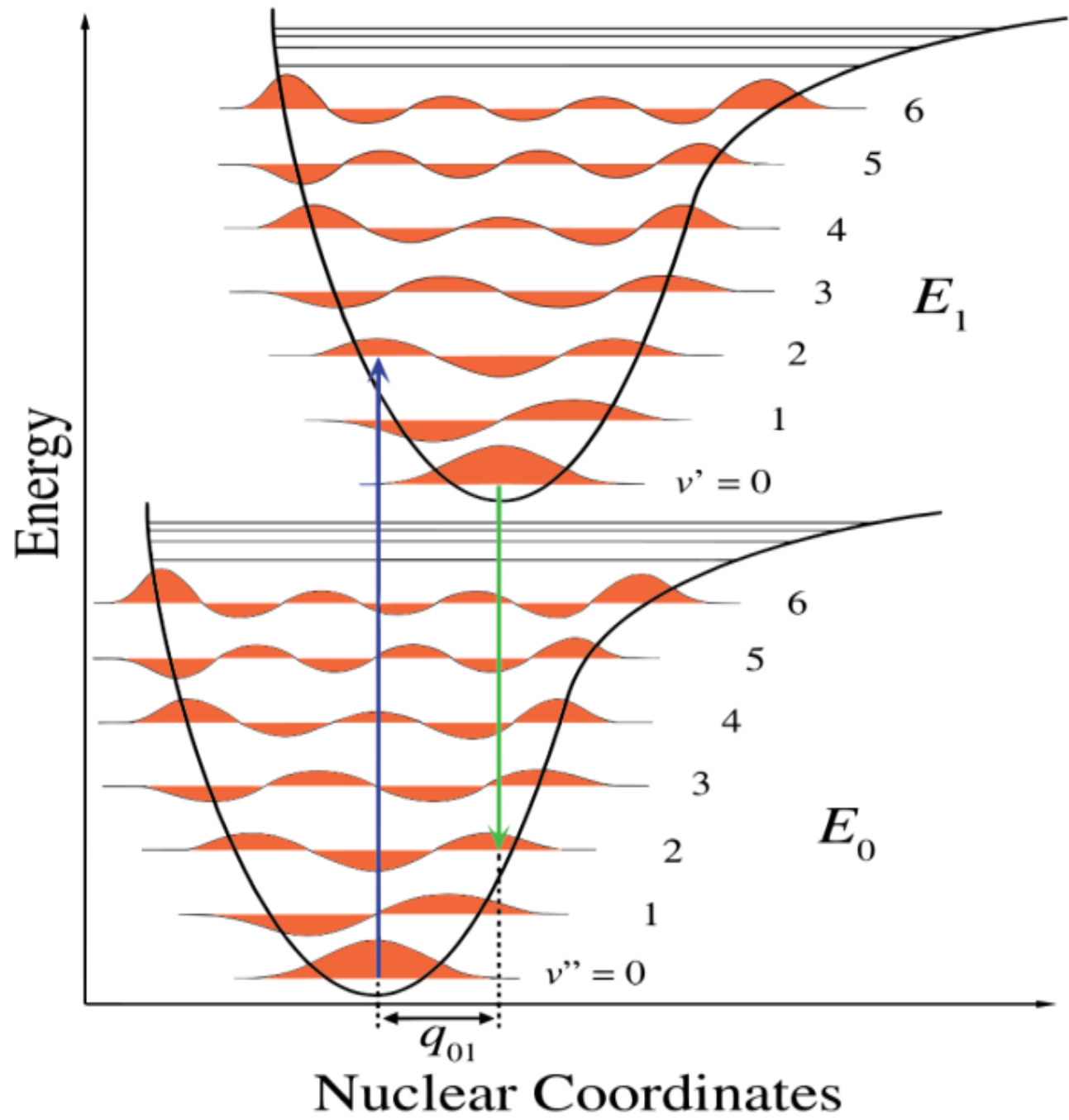

Figure 3.1. Franck-Condon principle energy diagram taken from ref. [119]. Since electronic transitions are very fast compared with nuclear motions, vibrational levels are favored when they correspond to a minimal change in the nuclear coordinates. The potential wells are shown favoring transitions between $v=0$ and $v=2$. 


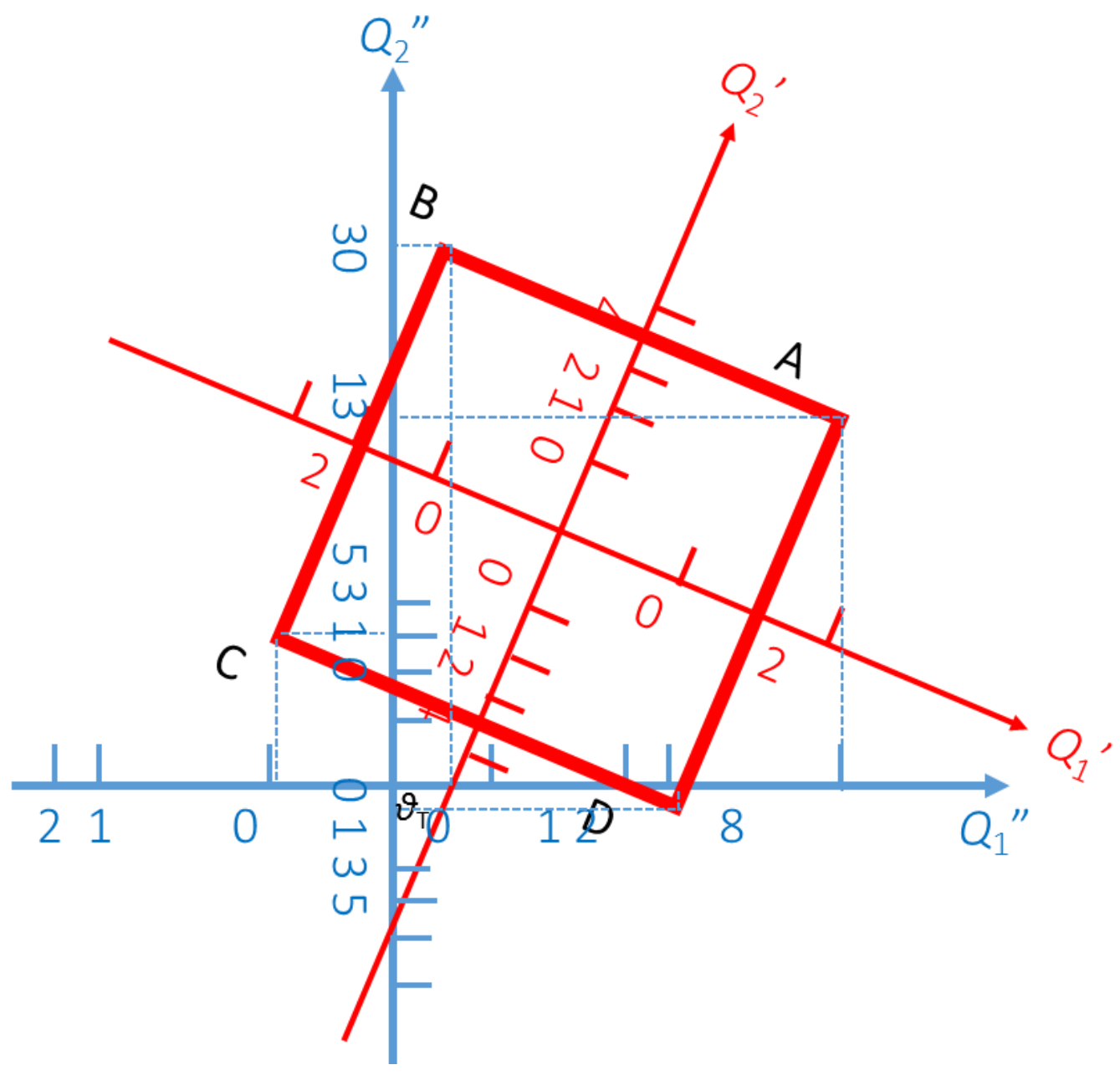

Figure 3.2. Duschinsky mixing between to normal modes can be presented as a rotation of the vibrational normal coordinates. $Q=\frac{1}{2 \pi} \sqrt{\frac{2 h}{v \mu}\left(v+\frac{1}{2}\right)}$, where $Q$ "' and $Q$ ' are the normal coordinates of the ground state and the excited state, respectively. $h$ is the Planck's constant, $\mu$ is the reduced mass, and $v$ is the vibrational quantum number. Adapted from ref. [121]. 


\section{CHAPTER IV}

\section{DISPERSED FLUORESCENCE SPECTROSCOPY OF JET-COOLED ISOBUTOXY AND 2-METHYL-1-BUTOXY RADICALS}

\subsection{Introduction}

Alkoxy radicals are important reaction intermediates in the combustion of fossil fuels ${ }^{3}$ and the atmosphere, especially in the troposphere. ${ }^{1,2}$ Extensive investigations have been carried out to explore the chemical kinetics involving alkoxy radicals. ${ }^{6,124-127}$ In the atmosphere, alkoxy radicals are formed in the degradation of volatile organic compounds (VOCs) typically initiated by reaction with an $\mathrm{OH}$ radical and in the presence of $\mathrm{NO}_{\mathrm{x}}$. They can undergo several reaction paths: reaction with $\mathrm{O}_{2}$, unimolecular dissociation (decomposition by $\beta \mathrm{C}-\mathrm{C}$ bond fission), and isomerization, for example, via a 1,5 $\mathrm{H}$-shift. Alkoxy radicals, therefore, constitute a critical branching point in the oxidation of VOCs.

Most alkoxy radicals have multiple structural and conformational isomers. It is well known that rate constants of alkoxy reactions can be highly sensitive to their conformations in the ground and excited states. For instance, the energy barrier for the isomerization of ground-state 1-butoxy (a 1,5 H-shift) is $3.6 \mathrm{kcal} / \mathrm{mol}$ lower than decomposition, whereas the barrier of isobutoxy isomerization (a 1,4 H-shift) is $10.6 \mathrm{kcal} / \mathrm{mol}$ higher than that of decomposition. This is due to the fact that the isomerization of isobutoxy occurs via a six- 
membered transition state, while the transition state of 1 - butoxy isomerization contains a five-membered ring that has higher strain energy and is less stable. Eventual stable products of particular hydrocarbon oxidation are determined by the competition between various potential reaction pathways of alkoxy radicals. Conformation-specific studies of alkoxy radicals, especially that of the correlation between their structures and their reactivities, are, therefore, of significant importance to atmospheric chemistry.

Previously, Jin et al..$^{34}$ reported dispersed fluorescence (DF) spectra of 1- and 2butoxies, in which vibrational structure of both the ground electronic $(\tilde{X})$ state and the first excited electronic ( $\tilde{A}$ ) state revealed itself in the DF spectra, along with the energy separation between the zero-point levels of these two states $\Delta E^{\tilde{A}-\tilde{X}}$. Significant differences are observable when the DF spectra of these two isomers are compared: in the DF spectra of 2-butoxy, there are fewer individual lines in the low-frequency region and lines become broader and more congested in the high-frequency region when compared to 1-butoxy. Such differences are attributed to the smaller $\tilde{A}-\tilde{X}$ separation for 2-butoxy than 1-butoxy, which was also used to explain the more irregular vibrational progressions in 2-butoxy.

Recently, our group observed the DF spectra of t-butoxy. Because of its high symmetry $\left(C_{3 v}\right)$, the ground electronic state of t-butoxy is orbitally doubly degenerate, albeit split by the spin-orbit interaction to two components $\left(\tilde{X}^{2} \mathrm{E}_{3 / 2}\right.$ and $\left.\tilde{X}^{2} \mathrm{E}_{1 / 2}\right)$ with their zero-point levels separated by $36 \mathrm{~cm}^{-1}$. The ground state of t-butoxy is also subject to the Jahn-Teller (JT) effect that couples the two degenerate vibronic state $\left|e_{\mathrm{x}}\right\rangle$ and $\left|\mathrm{ev}_{\mathrm{y}}\right\rangle$. Despite such complications, it was possible to assign almost all observed DF lines aided by vibrational frequencies and Franck-Condon factors (FCFs) calculated by the complete active space self-consistent field (CASSCF) method. 
It is therefore of interest to obtain the DF spectra of isobutoxy (2-methyl-1propoxy), the first target molecule of the present work, and make comparison with the other three isomers. Experimentally observed DF spectra elucidate the energy level structure of the $\tilde{X}$ and $\tilde{A}$ states and can be used to test the conformational assignment of the laserinduced fluorescence (LIF) spectrum previously reported by Liang et al. ${ }^{29}$ in which five vibronic bands of the $\tilde{B} \leftarrow \tilde{X}$ transition were observed. Because no rotational structure was resolved in the LIF spectrum, the conformational and vibrational assignments completely relied on ab initio calculations. Comparing between DF spectra obtained by pumping different vibronic bands in the LIF spectrum, one can differentiate LIF peaks of different conformers. Furthermore, comparison between the experimentally obtained transition frequencies in DF spectra and the calculated ground-state vibrational frequencies of different conformers allows a more reliable conformational assignment than that based on the comparison between LIF spectra and the calculated excited-state vibrational frequencies because calculations of ground-state vibrational frequencies are in general more accurate than the excited state. The DF spectra may also reveal the $\tilde{A}-\tilde{X}$ separation directly.

Chemical reactivities and branching ratios can also be affected by methyl substitution. In addition to isobutoxy, Ref. [29] reported the LIF spectrum of 2-methyl-1butoxy. Because of the longer carbon-chain than isobutoxy, a six-membered transition state for 1,5 H-shift is possible for 2-methyl-1-butoxy. Different branching ratios between the different reaction pathways are therefore expected for 2-methyl-1-butoxy and isobutoxy. It would also be interesting to compare the energy level structure of 2-methyl-1-butoxy to that of 1-butoxy and 2-butoxy, which may provide more information that will help explain 
the difference between their DF spectra. DF spectra of 2-methyl-1-butoxy were therefore obtained in the present work as well.

\subsection{Quantum Chemical Calculations}

Previously, geometry optimization and frequency calculation of both the $\tilde{X}$ and $\tilde{B}$ states of different conformers of isobutoxy and 2-methyl-1-butoxy radicals were performed using the Density Functional Theory (DFT) and CASSCF methods and the results were used for assignment of LIF spectra. ${ }^{29} \mathrm{We}$ have carried out same quantum chemical calculations in order to predict the Franck-Condon factors (FCFs) and simulate the $\tilde{B}-\tilde{X}$ and $\tilde{B}-\tilde{A}$ DF spectra. It was found that simulation using the DFT results reproduce the experimental spectra significantly better than the CASSCF results. All simulations reported in the present paper, therefore, use the DFT-calculated results at the B3LYP/6-31+G(d) level of theory for the $\tilde{X}$-state. The $\tilde{B}$ state geometry and vibrations were calculated using the Configuration Interaction Singles (CIS) method with the 6-31+G(d) basis set.

In the DF spectra, the energy separation between the $\tilde{X}$ and $\tilde{A}$ states $\left(\Delta E^{\tilde{A}-\tilde{X}}\right)$ may be observed directly (section 4.1.3). For molecules with $C_{s}$ symmetry, this separation can be predicted from quantum chemical calculations by demanding the unpaired electron occupy either of the $2 \mathrm{p} \pi$ orbitals localized at the oxygen atom: an unpaired electron in the $p_{x}$ orbital (within the $C_{s}$ plane) corresponds to an $\mathrm{A}^{\prime}$ state, while the $p_{y}$ orbital (out of the $C_{s}$ plane) is half-filled in an A" state. For isobutoxy, only one of its two conformers has $C_{s}$ symmetry. The calculations predict that its ground electronic $(\tilde{X})$ state has A" symmetry whereas the first excited state $(\tilde{A})$ is of A' symmetry. The energy separation between these two states has been found to be $401 \mathrm{~cm}^{-1}$ without zero-point energy (ZPE) corrections. The 2-methyl-1-butoxy radical does not have $C_{s}$ conformers. Hence no calculation of its $\Delta E^{\tilde{A}-\tilde{X}}$ 
was attempted.

All DFT and CIS calculations were performed using the Gaussian09 package. ${ }^{128}$ FCFs and transition intensities were predicted using the ezSpectrum software. ${ }^{121}$ Due to the significant difference between the $\tilde{X} / \tilde{A}$ and the $\tilde{B}$ state geometries, especially the large increase in the $\mathrm{CO}$ bond length upon excitation (e.g., from 1.37 to $1.56 \AA$ for isobutoxy), the Duschinsky rotation was taken into account in simulating the DF spectra. ${ }^{122,123,129}$

\subsection{Results}

DF spectra were obtained by pumping four vibronic bands of isobutoxy in its LIF spectrum (Bands A-D) and two LIF bands of 2-methyl-1-butoxy radicals (Bands A and B). The LIF bands are labeled following ref. [29] Their transition frequencies are listed in Table 4.1.

\subsubsection{Isobutoxy}

\subsubsection{Conformational Assignment}

DF spectra of isobutoxy are shown in Figure 4.1. Spectra obtained by pumping Band B, C, and D are similar to one another, all dominated by a vibrational progression of strong sharp peaks separated by $\sim 1000 \mathrm{~cm}^{-1}$. DF spectrum pumping Band A is significantly different. Although it also contains a progression, each member of the progression consists of multiple strong peaks. Furthermore, the first strong peak with a nonzero redshift (the second in the vibrational progression, i.e., $v=1$ ) in the DF spectrum obtained by pumping band $\mathrm{B}$ does not match any of the peaks in the second member of the progression that is seen in the DF spectrum obtained by pumping band A (Figure 4.5). Bands B-D and band 
A are therefore assigned to different conformers of the isobutoxy radical.

Isobutoxy has two stable conformers, with $C_{s}$ and $C_{1}$ symmetry. In Figure 4.2, simulated DF spectra of the $\tilde{B} \rightarrow \tilde{X}$ transitions of the two conformers are compared with the spectra that were obtained experimentally by pumping bands $\mathrm{A}$ and $\mathrm{B}$ in the LIF spectrum. Although the DF spectrum obtained by pumping band A (at $28091 \mathrm{~cm}^{-1}$ ) is relatively more complicated, an excellent match has been found between the experimental spectrum and the one simulated using the calculated vibrational frequencies and FCFs of the $C_{s}$ conformer. The simulated spectrum for the $C_{1}$ conformer reproduces the strong features in the experimental spectrum obtained by pumping band $\mathrm{B}$ (at $28787 \mathrm{~cm}^{-1}$ ). A better match could be reached if anharmonicity was taken into account (see below). On the basis of the comparison between the experimental and simulated spectra, bands A and B (the first two LIF peaks of isobutoxy) are assigned as the origin bands of the $C_{s}$ and $C_{1}$ conformers, respectively.

\subsubsection{Vibrational Assignment}

DF spectra obtained by pumping bands A and B are both dominated by strong vibrational progressions starting from the zero redshifts. For the spectrum obtained by pumping band $\mathrm{B}$ (origin band of the $C_{l}$ conformer), a linear fit of the ground-state vibrational energy level separation $[\Delta G(v)]$ yields a harmonic frequency of $\omega_{e}=1060 \pm 5 \mathrm{~cm}^{-1}$ and an anharmonic parameter of $\omega_{e} \chi_{e}=5.6 \pm 0.8 \mathrm{~cm}^{-1}$ (Table 4.6 and Figure 4.6). In comparison, the calculated harmonic frequency of the $\mathrm{CO}$ stretch mode of the $C_{l}$ conformer is $1073 \mathrm{~cm}^{-1}$. The strong peaks in the spectrum are therefore assigned to the $\mathrm{CO}$ stretch progression. This is consistent with the prediction that the DF spectra of alkoxy radicals have strong CO stretch 
transitions because of the elongation of the $\mathrm{CO}$ bond upon $\tilde{B} \leftarrow \tilde{X}$ excitation, which results in large FCFs for transitions to the CO stretch levels.

Bands $\mathrm{C}$ and $\mathrm{D}$ are also assigned to the $C_{l}$ conformer. Although all are dominated by a strong progression, the most noticeable difference between the DF spectrum obtained by pumping band $\mathrm{B}$ and those obtained by pumping bands $\mathrm{C}$ and $\mathrm{D}$ is that the strong progressions in the latter do not start from the zero redshift $\tilde{B} \rightarrow \tilde{X}$ origin band but rather from a low-frequency DF peak, labeled bands "c" and " $d$ " in Figure 4.1. Therefore, if the DF spectra pumping bands $\mathrm{C}$ and $\mathrm{D}$ are blueshifted by the vibrational frequencies of bands $\mathrm{c}$ and $\mathrm{d}$, respectively, the strong progressions in these two spectra will line up with the progression in the DF spectrum pumping band $\mathrm{B}$, as shown in Figure 4.3. It is worth noting that the vibrational interval of band $\mathrm{c}\left(129 \mathrm{~cm}^{-1}\right)$ is almost identical to that of band $\mathrm{C}$ (130 $\left.\mathrm{cm}^{-1}\right)$, while the vibrational interval of band $\mathrm{d}\left(258 \mathrm{~cm}^{-1}\right)$ is close to that of band $\mathrm{D}(238$ $\left.\mathrm{cm}^{-1}\right)$. Bands $\mathrm{c}$ and $\mathrm{C}$ are therefore assigned to the same vibrational mode $\left(-\mathrm{CH}_{2} \mathrm{O}\right.$ wagging; see Figure 4.7a) of the $\tilde{X}$ and the $\tilde{B}$ states, respectively. In the same way, bands d and $\mathrm{D}$ are assigned to the in-phase $\mathrm{CH}_{3}$ torsional mode of the two states (Figure $4.7 \mathrm{~b}$ ). Progressions in the DF spectra pumping bands $\mathrm{C}$ and D are combination bands of CO stretch and the pumped vibrational modes. Such a phenomenon was observed in the DF spectra of cyclohexoxy ${ }^{35}$ as well as 2-, 3-, and 4- methylcyclohexoxy ${ }^{93}$ (but not 1methylcyclohexoxy) and is an indication of large Duschinsky rotation upon excitation from the $\tilde{X}$ state to the $\tilde{B}$ state because of the large elongation of the CO bond and Duschinsky mixing between the $\mathrm{CO}$ stretch and certain non-CO stretch vibrational modes. ${ }^{122,123}$

For the DF spectrum obtained by pumping the $C_{s}$ conformer origin band (band A), each member of the vibrational progression consists of multiple strong peaks. The second 
member of the $\mathrm{CO}$ stretch progression $(v=1)$ consists of two strong peaks that are reproduced in the simulation as well as several weaker peaks. Inspection of the calculated vibrational modes shows that the two strong peaks (1010 and $1078 \mathrm{~cm}^{-1}$, labeled "y" and " $z$ " in Figure 4.2) can be assigned to vibrational modes with strong CO stretch character but mixed with the umbrella modes $\left(v_{14}\right.$ and $\left.v_{13}\right)$. The relatively weak peak at $940 \mathrm{~cm}^{-1}$ (peak $\mathrm{x}$ in Figure 4.2) is attributed to a vibrational mode that mixes the $\mathrm{CH}_{3}$ scissor and the $\mathrm{C}-\mathrm{C}$ stretch $\left(v_{15}\right)$, while the weak peak at $824 \mathrm{~cm}^{-1}$ (peak "w" in Figure 4.2 and not reproduced in simulation) is attributed to the umbrella mode involving all four carbon atoms $\left(v_{16}\right)$. (See Figure 4.8 for vibrational modes and calculated frequencies.) DF peaks in other members of the progression can be assigned to overtones and combinations of these four vibrational modes. Weak transitions between members of the $\mathrm{CO}$ stretch progression are combination bands of $\mathrm{CO}$ stretch modes and other low-frequency vibrational modes according to simulation.

Frequencies of strong transitions in the DF spectra of isobutoxy are summarized in Tables 4.2 and 4.3 and compared with the calculated values. Vibrational modes observed in the DF spectra are illustrated in Figures 4.7 and 4.8 for the $C_{1}$ and $C_{s}$ conformers, respectively.

\subsubsection{3 $\tilde{A}-\tilde{X}$ Separation}

When the origin band of the $C_{s}$ conformer (band A) is pumped, there is a peak at a 426 $\mathrm{cm}^{-1}$ redshift in the DF spectrum that is not reproduced in the simulated $\tilde{B} \rightarrow \tilde{X}$ spectrum (Figure 4.2). DFT calculations predict an $\tilde{A}-\tilde{X}$ separation of $401 \mathrm{~cm}^{-1}$ for the $C_{s}$ conformer. The DF peak at $426 \mathrm{~cm}^{-1}$ is therefore assigned to the origin band of the $\tilde{B} \rightarrow \tilde{A}$ transition 
of the $C_{s}$ conformer. Similarly, the weak peak at a $373 \mathrm{~cm}^{-1}$ redshift in the DF spectrum obtained by pumping band $\mathrm{B}$ is assigned to the origin band of the $\tilde{B} \rightarrow \tilde{A}$ transition of the $C_{l}$ conformer.

\subsubsection{2-Methyl-1-butoxy}

DF spectra were recorded by pumping bands A (at $28097 \mathrm{~cm}^{-1}$ ) and B (at $28721 \mathrm{~cm}^{-1}$ ) in the LIF spectrum of 2-methyl-1-butoxy (Figure 4.4). Both spectra are dominated by two strong progressions. The redshift frequencies, however, are different for the two spectra, which suggests the assignment of bands A and B to two different conformers.

2-Methyl-1-butoxy has nine conformers. ${ }^{29}$ The conformers are named according to their $\mathrm{OC}_{2} \mathrm{C}_{3} \mathrm{C}_{4}$ and $\mathrm{C}_{2} \mathrm{C}_{3} \mathrm{C}_{4} \mathrm{C}_{5}$ dihedral angles. DFT calculations predict that the two lowest-energy ones are the $G_{1} T_{2}$ and the $T_{1} T_{2}$ conformers, with the latter being 0.05 $\mathrm{kcal} / \mathrm{mol}$ higher in energy than the former. The next lowest-energy conformer $\left(\mathrm{T}_{1} \mathrm{G}_{2}{ }^{\prime}\right)$ is significantly higher in energy $\left(0.24 \mathrm{kcal} / \mathrm{mol}\right.$ higher than $\left.\mathrm{G}_{1} \mathrm{~T}_{2}\right)$. Hence, the $\tilde{B} \rightarrow \tilde{X} \mathrm{DF}$ spectra of $G_{1} T_{2}$ and $T_{1} T_{2}$ conformers were simulated and are compared with experimentally obtained spectra in Figure 4.4. Experimentally, broadening and congestion of lines are more significant in the DF spectrum obtained by pumping band A than that for band $B$. In simulations, the spectrum of $T_{1} T_{2}$ has strong transitions to two vibrational modes $\left(v_{30}\right.$ and $\left.v_{28}\right)$, both of which have $\mathrm{CO}$ stretch character but are mixed with the $\mathrm{C}-\mathrm{C}$ stretch modes. These two transitions are consistent with the two partially resolved peaks at 1017 and $1078 \mathrm{~cm}^{-1}$ observed in the DF spectrum obtained by pumping band A (labeled y and $\mathrm{z}$ in Figure 4.4). On the contrary, in the simulated $\mathrm{DF}$ spectrum of $\mathrm{G}_{1} \mathrm{~T}_{2}$, only a single strong $\mathrm{CO}$ stretch transition $\left(v_{28}\right)$ is predicted for each member of the progression, 
corresponding to the strong peak at $1053 \mathrm{~cm}^{-1}$ in the DF spectrum obtained by pumping band $B$. Band $A$ is therefore assigned to the $T_{1} T_{2}$ conformer and band $B$ to the $G_{1} T_{2}$ conformer. In both cases, DFT calculations predict $\mathrm{CO}$ stretch frequencies with high accuracy. For the $\mathrm{G}_{1} \mathrm{~T}_{2}$ conformer, a linear fit of the first four ground-state vibrational energy levels yields a harmonic frequency of $\omega_{e}=1075 \pm 8 \mathrm{~cm}^{-1}$ and an anharmonic parameter of $\omega_{e} \chi_{e}=9.2 \pm 1.5 \mathrm{~cm}^{-1}$ (Table 4.7 and Figure 4.9), compared to the calculated value of $1053 \mathrm{~cm}^{-1}$.

Members of the second (higher-frequency) progressions in DF spectra of both conformers of 2-methyl-1-butoxy have a constant separation with respect to those of the $\mathrm{CO}$ stretch progression. They are assigned to combination bands of the $\mathrm{CO}$ stretch and other non-CO stretch modes based on FCF calculations, although their intensities are underestimated by the calculations. According to the FCF calculations, the strongest transitions in the second progression in the $\mathrm{DF}$ spectrum of the $\mathrm{T}_{1} \mathrm{~T}_{2}$ conformer are combination bands of the $\mathrm{CO}$ stretch and the $\mathrm{C}_{2} \mathrm{C}_{3} \mathrm{C}_{4}$ bending mode $\left(v_{43}\right)$, while those in the DF spectrum of the $\mathrm{G}_{1} \mathrm{~T}_{2}$ conformer are combination bands of the $\mathrm{CO}$ stretch and methyl torsional modes ( $v_{43}$ and $v_{42}$ ). (See Figures 4.10 and 4.11 for vibrational modes and calculated frequencies.)

Frequencies of strong transitions in the DF spectra of 2- methyl-1-butoxy are summarized in Tables 4.4 and 4.5 and compared with the calculated values. Vibrational modes observed in the DF spectra are illustrated in Figures 4.10 and 4.11 for the $\mathrm{T}_{1} \mathrm{~T}_{2}$ and $\mathrm{G}_{1} \mathrm{~T}_{2}$ conformers, respectively.

\subsection{Discussion}


In the present work, for both isobutoxy and 2-methyl-1-butoxy radicals, bands A and B in the LIF spectra are assigned to origin bands of two different conformers because of the difference in the DF spectra obtained by pumping these two bands. On the contrary, Liang et al. ${ }^{29}$ assigned all vibronic peaks observed in the LIF spectrum of each radical to a single conformer. In their work, A bands were assigned to the $\tilde{B} \leftarrow \tilde{X}$ origin bands of the lowestenergy conformers (the $C_{l}$ conformer of isobutoxy and the $\mathrm{G}_{1} \mathrm{~T}_{2}$ conformer of 2-methyl-1butoxy), while B bands were assigned to transitions to the $\mathrm{CO}$ stretch fundamental level of the $\tilde{B}$ state. These assignments were made based on two facts: first, the differences between the $\tilde{B} \leftarrow \tilde{X}$ transition frequencies for the two lowest-energy conformers calculated by the CASSCF method are significantly smaller than the separation between bands A and B. Such a discrepancy, therefore, does not support the assignment of bands A and B to two different conformers. Second, the separations between bands A and B $\left(697 \mathrm{~cm}^{-1}\right.$ for isobutoxy and $624 \mathrm{~cm}^{-1}$ for 2-methyl-1-butoxy) are close to the calculated excited-state $\mathrm{CO}$ stretch frequencies $\left(674 \mathrm{~cm}^{-1}\right.$ for isobutoxy and $663 \mathrm{~cm}^{-1}$ for 2-methyl-1-butoxy). Although the unambiguous assignment is typically not possible until rotationally resolved LIF spectra are obtained, the experimentally observed DF spectra provide a good foundation for the two-conformer assignment in the present work. We would also like to point out that CASSCF calculations provided extremely accurate predictions of the excitedstate CO stretch frequencies for t-butoxy, ${ }^{130} 544 \mathrm{~cm}^{-1}$ (calculated) vs. $543.9 \mathrm{~cm}^{-1}$ (experimental), because this state, unlike the ground electronic $\left(\tilde{X}^{2} \mathrm{E}\right)$ state, is not subject to strong vibronic interaction. In the present case, the calculated CO stretch frequencies of the isobutoxy and 2-methyl-1-butoxy radicals may not be close enough to the separations between bands $\mathrm{A}$ and $\mathrm{B}$, for both radicals, to serve as a strong support to the single- 
conformer assignment. Furthermore, prediction of the adiabatic $\tilde{B}-\tilde{X}$ transition frequencies by the CASSCF method might not be accurate enough to guide the conformational assignment because the lower state $(\tilde{X})$ is semi-degenerate with and strongly perturbed by the first excited state $(\tilde{A})$.

Reassignment of B bands from transitions to the $\mathrm{CO}$ stretch levels of the lowestenergy conformers to the origin bands of the second-lowest-energy conformers necessitates the reassignment of bands $\mathrm{C}, \mathrm{D}$, and $\mathrm{E}$ of isobutoxy. As discussed in section 4.1.2, bands $\mathrm{C}$ and $\mathrm{D}$ in the LIF spectrum are transitions to the same vibrational modes as bands $\mathrm{c}$ and $\mathrm{d}$ in the DF spectrum, respectively, although their relative frequencies might be slightly different because bands $\mathrm{C}$ and $\mathrm{D}$ correspond to vibrational modes in the $\tilde{B}$ state while bands c and d correspond to vibrational modes in the $\tilde{X}$ state. In comparison to DFT (for ground state) and CASSCF and CIS (for excited state) calculations, band C (as well as band c) is assigned to the $-\mathrm{CH}_{2} \mathrm{O}$ wagging mode calculated to be $132 \mathrm{~cm}^{-1}$ by the CIS method. Although an antisymmetric mode, this is a pseudo-JT mode that couples the two lowest electronic ( $\tilde{A}$ and $\tilde{X}$ ) states, hence the observation of the LIF transition. Band $\mathrm{D}$ (as well as band d) is assigned to the in-phase torsional mode of the two methyl groups calculated to be $254 \mathrm{~cm}^{-1}$. Assignments of the LIF bands are summarized in Table 4.1. A DF spectrum pumping band $\mathrm{E}\left(\tilde{v}_{\mathrm{E}}=\tilde{v}_{\mathrm{B}}+428 \mathrm{~cm}^{-1}\right)$ was also obtained (Figure 4.12), which suggests that the carrier of band $\mathrm{E}$ is formaldehyde. It is well-known that formaldehyde can be generated via $\beta \mathrm{C}-\mathrm{C}$ bond fission of highly excited alkoxy radicals produced with $355 \mathrm{~nm}$ laser photolysis under jet-cooled conditions. ${ }^{131}$

Comparing between the two free radicals investigated in the present work, DF spectra of both conformers of isobutoxy contain sharp lines, while transitions in the DF 
spectra of 2- methyl-1-butoxy are broadened. Similarly, lines in the previously reported DF spectra of 2-butoxy were significantly broader than 1-butoxy, ${ }^{34}$ which was attributed to the smaller $\tilde{A}-\tilde{X}$ separation of 2-butoxy when compared to 1-butoxy, and hence stronger vibronic interaction between the $\tilde{A}$ - and $\tilde{X}$-states. In the present work, the $\tilde{A}-\tilde{X}$ separation has been determined to be $373 \mathrm{~cm}^{-1}$ for the $C_{l}$ conformer of the isobutoxy radical and 426 $\mathrm{cm}^{-1}$ for its $C_{s}$ conformer, both of which are larger than those of 1-butoxy conformers: 271, 292, and $129 \mathrm{~cm}^{-1}$ for its $\mathrm{T}_{1} \mathrm{G}_{2}, \mathrm{~T}_{1} \mathrm{~T}_{2}, \mathrm{G}_{1} \mathrm{~T}_{2}$ conformers, respectively. $\tilde{B} \rightarrow \tilde{A}$ transitions were not observed in the DF spectra of 2-methyl-1-butoxy probably because its $\tilde{A}-\tilde{X}$ separation is smaller than the experimental resolution, which implies that its $\tilde{A}-\tilde{X}$ separation is even smaller than that of the 2-butoxy radical $\left(55 \mathrm{~cm}^{-1}\right.$ for its $\mathrm{T}$ conformer). The addition of a methyl group to the isobutoxy radical, therefore, significantly reduces the energy separation and enhances vibronic interaction between the $\tilde{X}$ and $\tilde{A}$ states, which are characterized by different half-filled $\mathrm{p} \pi$ orbitals $\left(p_{x}\right.$ and $\left.p_{y}\right)$. The present results, therefore, support the hypothesis that line broadening in the DF spectra of alkoxy radicals is at least partially due to vibronic interaction between the nearly degenerate $\tilde{X}$ and $\tilde{A}$ states. DF spectra of both conformers of isobutoxy are simpler when compared to those of t-butoxy, ${ }^{120}$ which contain not only progressions of the $\mathrm{CO}$ stretch and its combination bands but also transitions to asymmetric vibrational levels that are usually forbidden by symmetry and allowed in the DF spectra of t-butoxy because of the JT effect. Recent high-resolution spectroscopy investigations revealed both similarities and differences between the rotational and fine structure of the JT molecules (e.g., t-butoxy) and pseudo-JT molecules (e.g., isobutoxy). ${ }^{33,132}$ It would be interesting to compare the vibronic structures of these two types of molecules. Such investigation, however, is out of the scope of the present 
dissertation.

\subsection{Conclusions}

DF spectra of isobutoxy and 2-methyl-1-butoxy radicals were obtained by pumping different vibronic bands in their LIF spectrum. In all cases, the DF spectra are dominated by $\mathrm{CO}$ stretch progressions when the origin bands are pumped and by the progression of combination bands of $\mathrm{CO}$ stretch and the pumped modes when other non-CO stretch bands are pumped. Simulated spectra using calculated vibrational frequencies and CFCs well reproduced the DF spectra. Differences tween DF spectra obtained by pumping different vibronic bands suggest that for both free radicals, at least two lowest-energy conformers are generated by $355 \mathrm{~nm}$ photolysis under jet-cooled conditions. 
Table 4.1. Vibronic bands in the LIF spectra of isobutoxy and 2-methyl-1-butoxy reported in reference [29] and their assignments based on DF spectra and calculations obtained in the present work.

\begin{tabular}{|c|c|c|c|c|c|}
\hline \multicolumn{6}{|c|}{ vibrational interval } \\
\hline radical & band & frequency & expt & calcd $^{\mathrm{a}}$ & assignment \\
\hline \multirow{5}{*}{ isobutoxy } & A & 28091 & 0 & & $v_{00}^{\tilde{B}} \leftarrow \tilde{\mathrm{X}}$ of $\mathrm{C}_{\mathrm{s}}$ conformer \\
\hline & B & 28787 & 0 & & $v_{00}^{\tilde{\mathrm{B}}} \leftarrow \tilde{\mathrm{X}}$ of $\mathrm{C}_{1}$ conformer \\
\hline & $\mathrm{C}$ & 28917 & $\tilde{v}_{B}+130$ & 132 & $-\mathrm{CH}_{2} \mathrm{O}$ wagging \\
\hline & $\mathrm{D}$ & 29025 & $\tilde{v}_{B}+238$ & 254 & in-phase methyl torsion \\
\hline & $\mathrm{E}$ & 29215 & & & $\mathrm{~b}$ \\
\hline \multirow{2}{*}{$\begin{array}{l}\text { 2-methyl- } \\
\text { 1-butoxy }\end{array}$} & A & 28097 & 0 & & $v_{00}^{\tilde{\mathrm{B}}} \leftarrow \tilde{\mathrm{X}}$ of $\mathrm{T}_{1} \mathrm{~T}_{2}$ conformer \\
\hline & B & 28271 & 0 & & $v_{00}^{\tilde{B}} \leftarrow \tilde{X}$ of $\mathrm{G}_{1} \mathrm{~T}_{2}$ conformer \\
\hline
\end{tabular}

${ }^{\text {a }}$ Calculated at the CIS/6-31+G(d) level of theory.

${ }^{\mathrm{b}}$ Transition of formaldehyde. 
Table 4.2. Assignment of transitions in the DF spectrum of the $C_{s}$ conformer of the isobutoxy radical by pumping its origin band A (Figure 4.1). ${ }^{\mathrm{a}}$

\begin{tabular}{|c|c|c|c|}
\hline expt & calcd & assignment & $\left|\left\langle\mathrm{v}^{\prime} \mid \mathrm{v}^{\prime \prime}\right\rangle\right|^{2}$ \\
\hline 0 & 0 & $0_{0}^{0}(\tilde{\mathrm{B}} \rightarrow \tilde{\mathrm{X}})$ & 0.0106 \\
\hline 222 & 224 & $v_{20}$ & 0.0011 \\
\hline 270 & 277 & $v_{19}$ & 0.0018 \\
\hline 426 & & $0_{0}^{0}(\tilde{\mathrm{B}} \rightarrow \tilde{\mathrm{A}})$ & \\
\hline $\begin{array}{l}601 \\
651\end{array}$ & & & \\
\hline 824 & 783 & $v_{16}$ & \\
\hline 940 & 943 & $v_{15}$ & 0.0039 \\
\hline 1010 & 1044 & $v_{14}$ & 0.0163 \\
\hline 1078 & 1123 & $v_{13}$ & 0.0100 \\
\hline 2025 & 2089 & $2 v_{14}$ & 0.0133 \\
\hline 2100 & 2168 & $v_{14}+v_{13}$ & 0.0165 \\
\hline 2169 & 2247 & $2 v_{13}$ & 0.0047 \\
\hline 3021 & 3134 & $3 v_{14}$ & 0.0076 \\
\hline 3110 & 3213 & $2 v_{14}+v_{13}$ & 0.0144 \\
\hline 3188 & 3290 & $v_{14}+2 v_{13}$ & 0.0082 \\
\hline 3271 & 3370 & $3 v_{13}$ & 0.0014 \\
\hline 4038 & 4177 & $4 v_{14}$ & 0.0035 \\
\hline 4122 & 4256 & $3 v_{14}+v_{13}$ & 0.0088 \\
\hline 4197 & 4335 & $2 v_{14}+2 v_{13}$ & 0.0077 \\
\hline 4283 & 4414 & $v_{14}+3 v_{13}$ & 0.0027 \\
\hline 4365 & 4493 & $4 v_{14}$ & 0.0003 \\
\hline
\end{tabular}

a Experimentally obtained vibrational intervals are compared to calculated $\tilde{X}$-state vibrational frequencies (in $\mathrm{cm}^{-1}$ ). Vibrational modes are numbered following the Herzberg convention. FCFs for the $\tilde{B} \rightarrow \tilde{X}$ transitions are calculated using the ezSpectrum software with a threshold of 0.0001 . 
Table 4.3. Assignment of transitions in the DF spectrum of the $C_{1}$ conformer of the isobutoxy radical by pumping its origin band B (Figure 4.1).

\begin{tabular}{cccc}
\hline expt & calcd & assignment & $\left|\left\langle\mathrm{v}^{\prime} \mid \mathrm{v}^{\prime \prime}\right\rangle\right|^{2}$ \\
\hline 0 & 0 & $0_{0}^{0}(\tilde{\mathrm{B}} \rightarrow \tilde{\mathrm{X}})$ & 0.0144 \\
370 & & $0_{0}^{0}(\tilde{\mathrm{B}} \rightarrow \tilde{\mathrm{A}})$ & 0.0006 \\
421 & & & \\
712 & & & \\
1049 & 1073 & $v_{23}$ & 0.0398 \\
2082 & 2145 & $2 v_{23}$ & 0.0582 \\
3114 & 3218 & $3 v_{23}$ & 0.0596 \\
4132 & 4291 & $4 v_{23}$ & 0.0481 \\
5133 & & & \\
\hline
\end{tabular}


Table 4.4. Assignment of transitions in the DF spectrum of the $\mathrm{T}_{1} \mathrm{~T}_{2}$ conformer of the 2methyl-1-butoxy radical by pumping its origin band A (Figure 4.4).

\begin{tabular}{|c|c|c|c|}
\hline expt & calcd & assignment & $\left|\left\langle\mathrm{v}^{\prime} \mid \mathrm{v}^{\prime \prime}\right\rangle\right|^{2}$ \\
\hline 0 & 0 & $0_{0}^{0}$ & 0.0133 \\
\hline 1017 & 1037 & $v_{30}$ & 0.0089 \\
\hline 1078 & 1082 & $v_{28}$ & 0.0266 \\
\hline \multicolumn{4}{|l|}{1718} \\
\hline & 2074 & $v_{30}$ & 0.0028 \\
\hline \multirow[t]{2}{*}{$2114^{\mathrm{a}}$} & 2120 & $v_{30}+v_{28}$ & 0.0189 \\
\hline & 2166 & $v_{28}$ & 0.0280 \\
\hline \multicolumn{4}{|l|}{2404} \\
\hline \multicolumn{4}{|l|}{2728} \\
\hline \multirow[t]{4}{*}{$3110^{\mathrm{a}}$} & 3112 & $2 v_{30}$ & 0.0006 \\
\hline & 3157 & $v_{30}+v_{28}$ & 0.0064 \\
\hline & 3203 & $v_{30}+v_{28}$ & 0.0210 \\
\hline & 3248 & $3 v_{28}$ & 0.0204 \\
\hline \multicolumn{4}{|l|}{3738} \\
\hline & 4194 & $3 v_{30}+v_{28}$ & 0.0014 \\
\hline \multirow[t]{3}{*}{$4257^{\mathrm{a}}$} & 4240 & $2 v_{30}+2 v_{28}$ & 0.0075 \\
\hline & 4285 & $v_{30}+3 v_{28}$ & 0.0161 \\
\hline & 4330 & $4 v_{28}$ & 0.0116 \\
\hline $5292^{\mathrm{a}}$ & & & \\
\hline
\end{tabular}

${ }^{a}$ Center frequency of partially resolved peaks. 
Table 4.5. Assignment of transitions in the DF spectrum of the $\mathrm{G}_{1} \mathrm{~T}_{2}$ conformer of the 2methyl-1-butoxy radical by pumping its origin band B (Figure 4.4).

\begin{tabular}{cccc}
\hline expt & calcd & assignment & $\left|\left\langle\mathrm{v}^{\prime} \mid \mathrm{v}^{\prime \prime}\right\rangle\right|^{2}$ \\
\hline 0 & 0 & $0_{0}^{0}$ & 0.0133 \\
1045 & 1074 & $v_{28}$ & 0.0367 \\
1323 & & & \\
2085 & 2147 & $2 v_{28}$ & 0.0538 \\
2356 & & & \\
3106 & 3220 & $3 v_{28}$ & 0.0554 \\
3369 & & & \\
4131 & 4294 & $4 v_{28}$ & 0.0450 \\
4381 & & & \\
5157 & & & \\
5371 & & & \\
\hline
\end{tabular}


Table 4.6. Experimentally determined ground-state vibrational energy levels $G(v)$ of the $\mathrm{CO}$ stretch progression (in $\mathrm{cm}^{-1}$ ) of the $C_{l}$ conformer of the isobutoxy radical. $\Delta G(v)=$ $G(v)-G(v-1)$.

\begin{tabular}{ccc}
\hline$v$ & $\mathrm{G}(v)$ & $\Delta \mathrm{G}(v)$ \\
\hline 0 & 0 & \\
1 & 1049 & 1049 \\
2 & 2082 & 1033 \\
3 & 3114 & 1032 \\
4 & 4132 & 1018 \\
5 & 5133 & 1001 \\
\hline
\end{tabular}

Table 4.7. Experimentally determined ground-state vibrational energy levels $G(v)$ of the $\mathrm{CO}$ stretch progression (in $\mathrm{cm}^{-1}$ ) of $\mathrm{G}_{1} \mathrm{~T}_{2}$ conformer of the 2-methyl-1-butoxy radical.

\begin{tabular}{ccc}
\hline$v$ & $\mathrm{G}(v)$ & $\Delta \mathrm{G}(v)$ \\
\hline 0 & 0 & \\
1 & 1045 & 1045 \\
2 & 2088 & 1043 \\
3 & 3135 & 1047 \\
4 & 4149 & 1014 \\
\hline
\end{tabular}




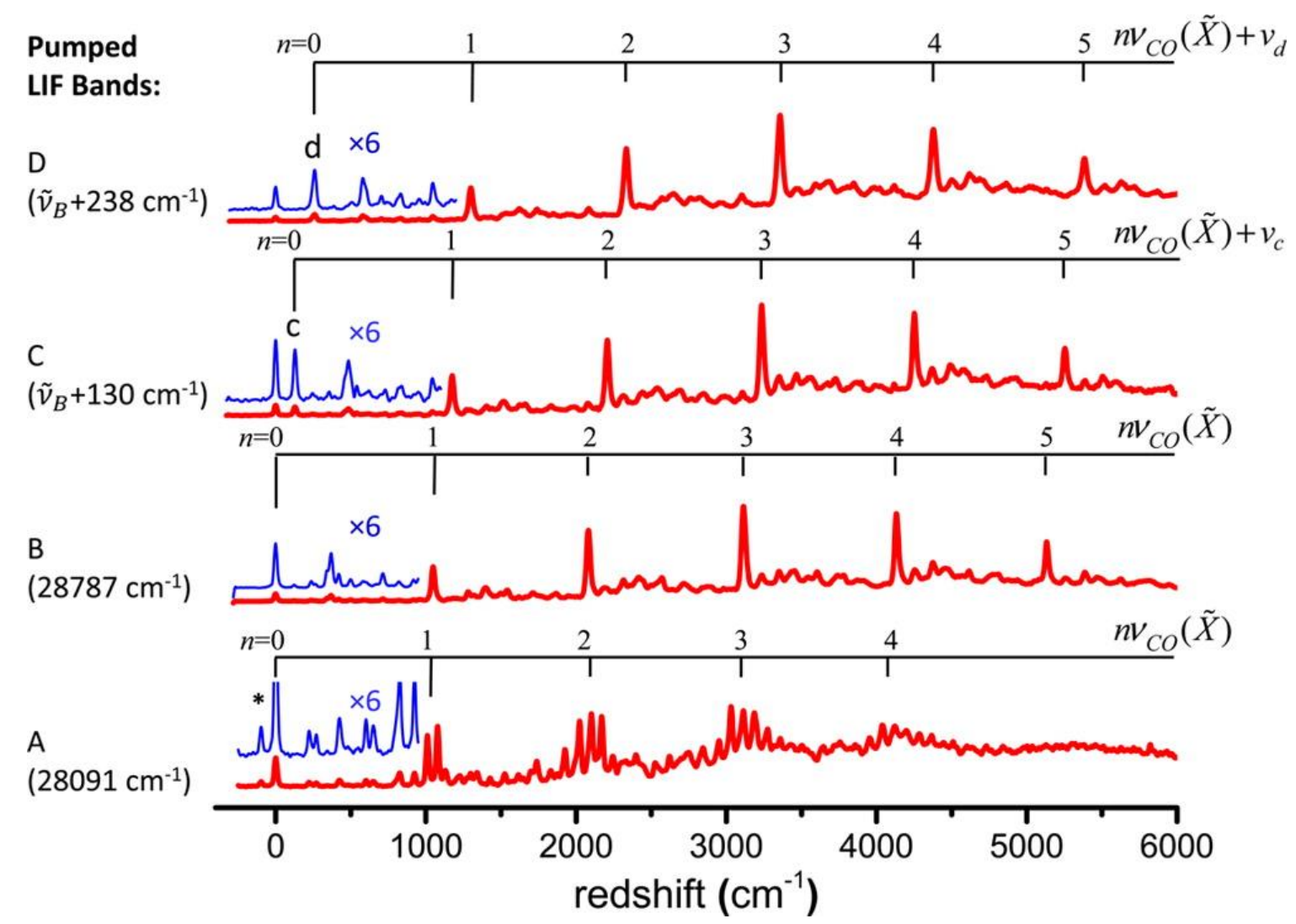

Figure 4.1. DF spectra of the isobutoxy radical. The asterisked peak is due to scattering of the $355 \mathrm{~nm}$ photolysis laser. Numbers in parentheses are LIF excitation frequencies. 


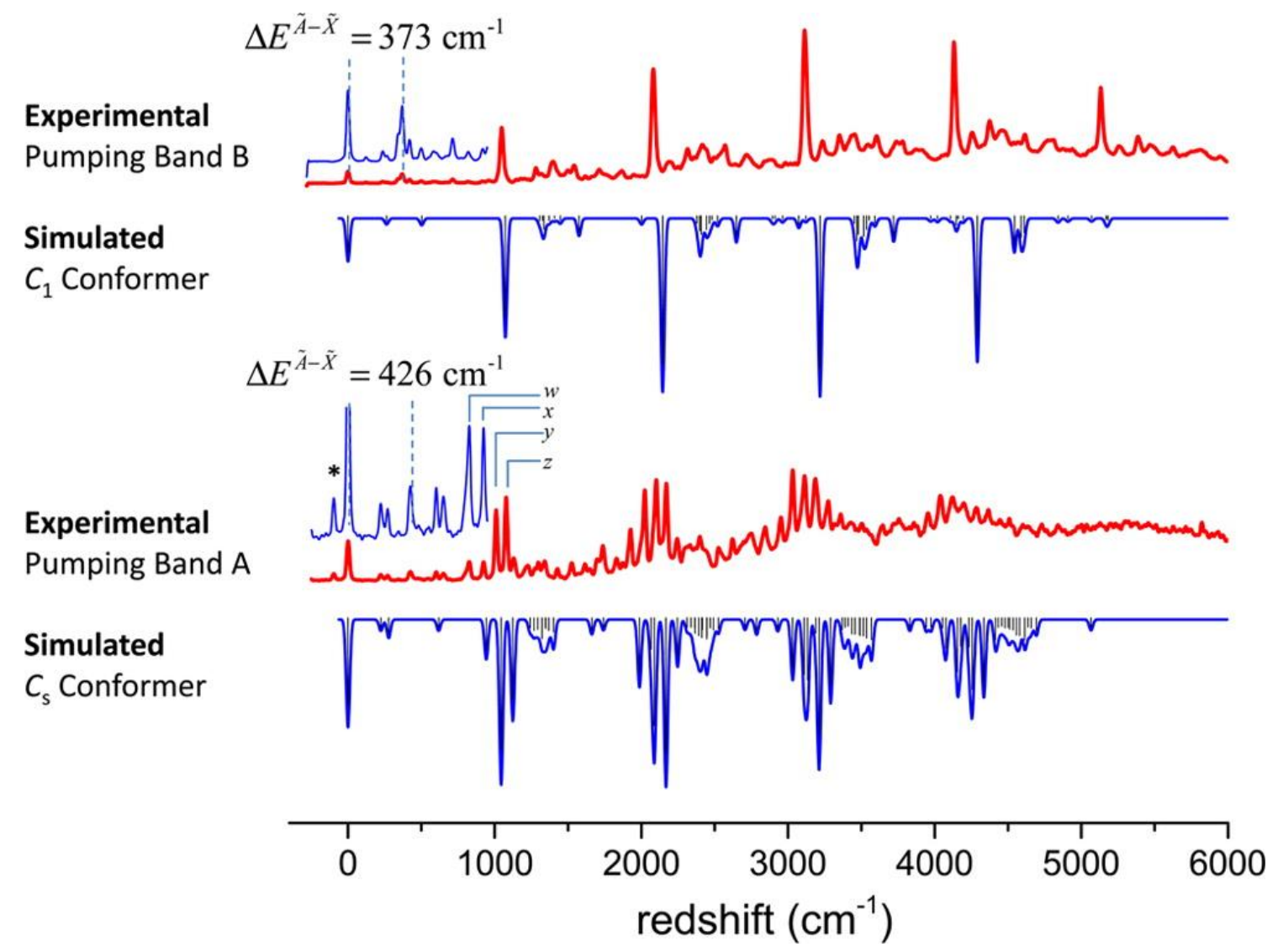

Figure 4.2. Comparison between the simulated DF spectra of the $C_{s}$ and $C_{l}$ conformers of the isobutoxy radical (blue) and the experimentally obtained ones by pumping bands $\mathrm{A}$ and $\mathrm{B}$ in the LIF spectrum (red). 


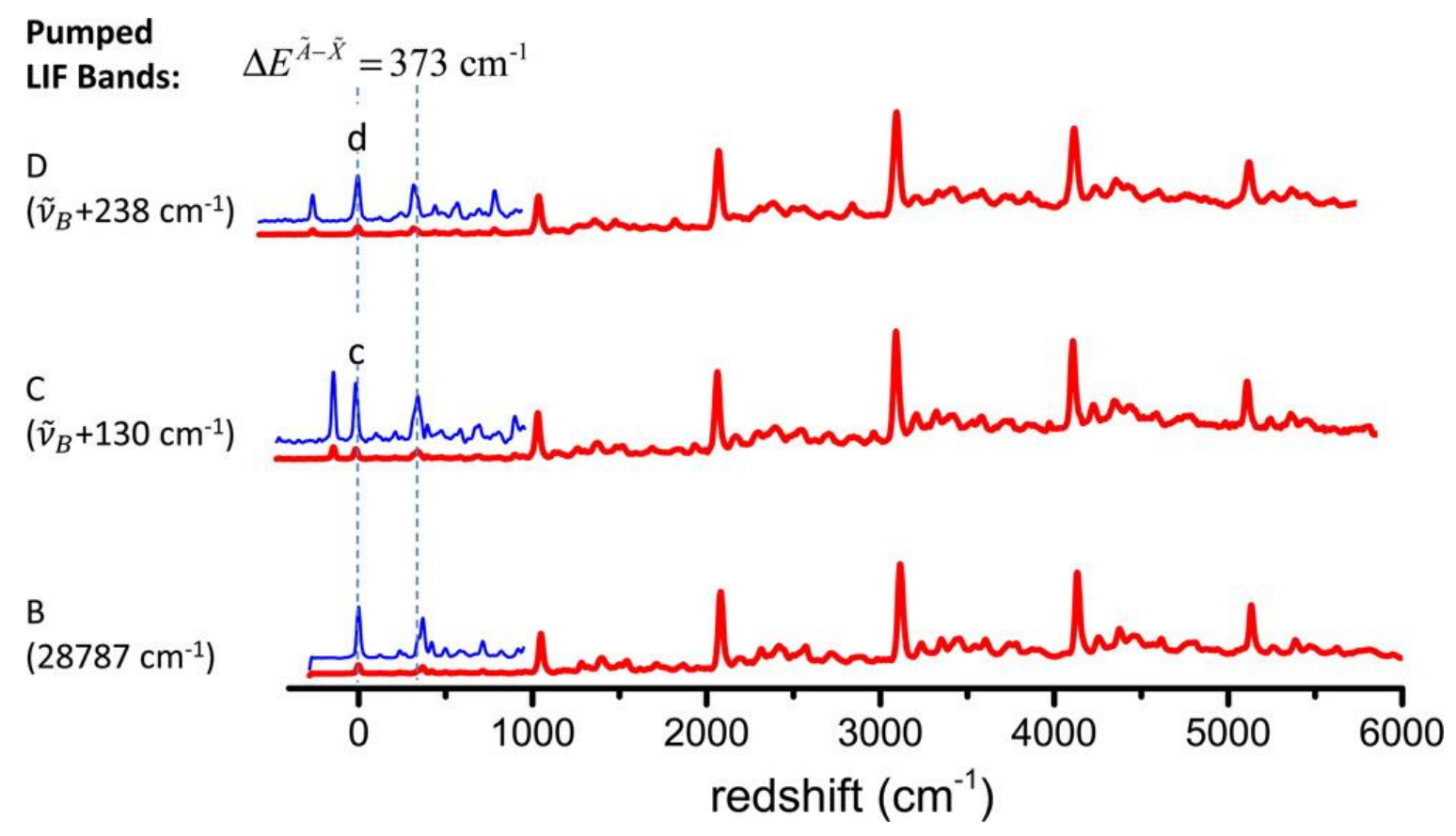

Figure 4.3. DF spectra of the isobutoxy radical obtained by pumping LIF bands B-D. The spectra obtained by pumping vibronic bands other than the origin (band B) are blue-shifted by the $\tilde{X}$-state vibrational frequency of the pumped mode so that the DF bands to the $\tilde{X}$-state vibrational levels are aligned. 


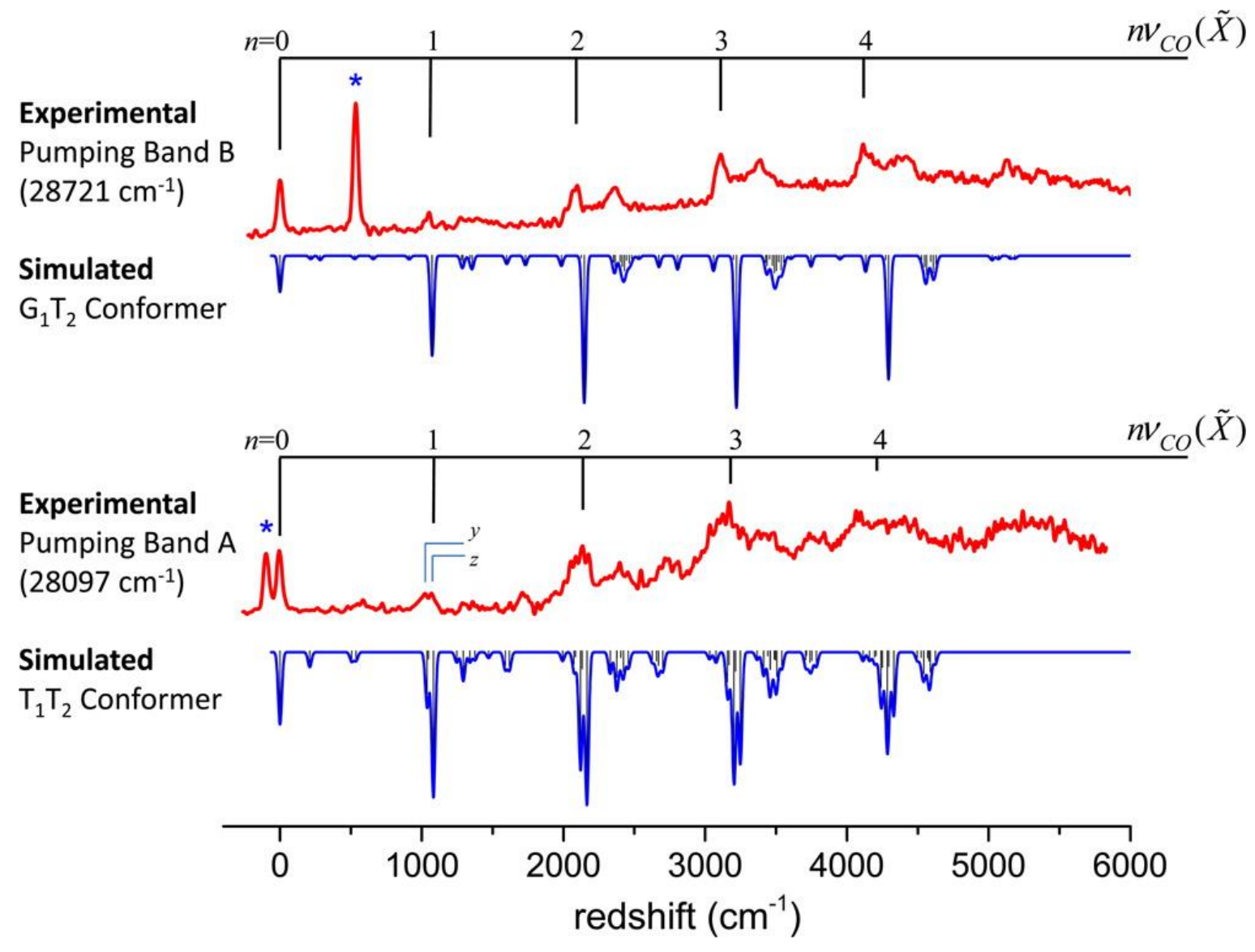

Figure 4.4. DF spectra of 2-methyl-1-butoxy radical (red) compared with simulation (blue). The asterisked peak is due to scattering of the $355 \mathrm{~nm}$ photolysis laser. 


\section{Pumped}

LIF Bands:

B

$\left(28788 \mathrm{~cm}^{-1}\right)$

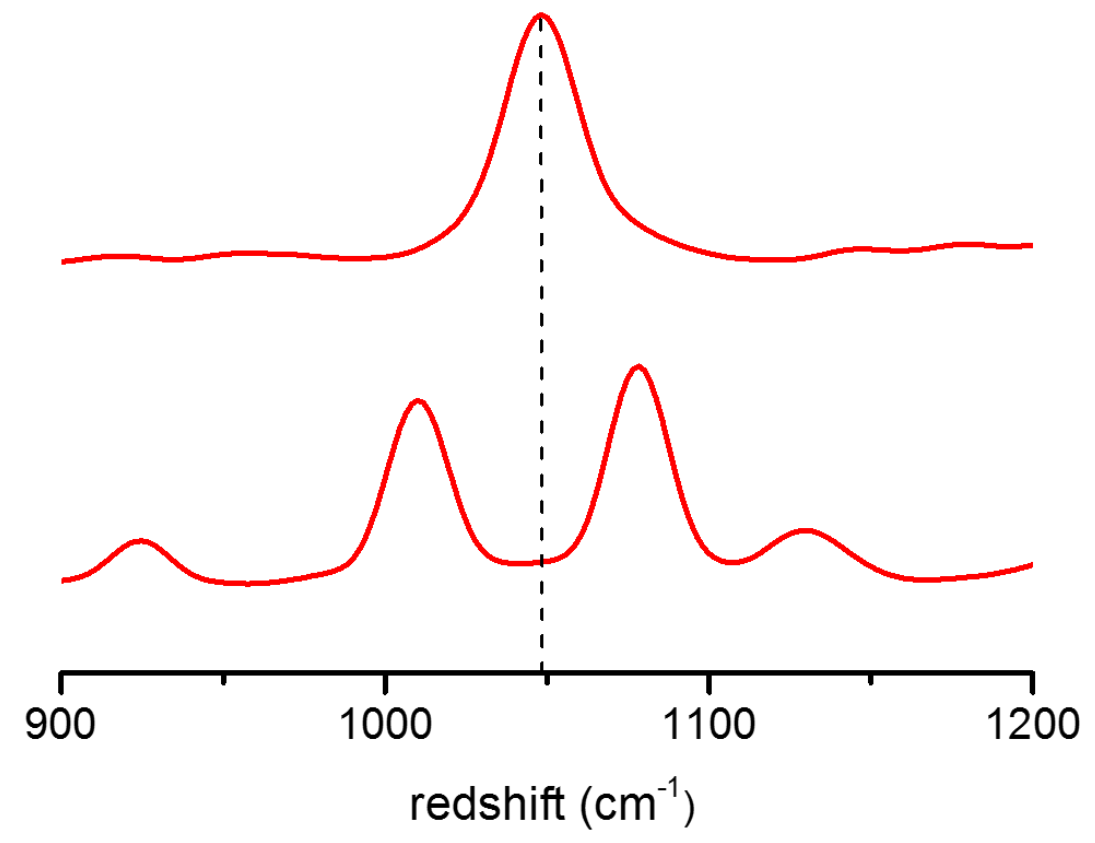

Figure 4.5. Comparison between the second members of the CO-stretch progression in the DF spectra of the isobutoxy radical by pumping Bands A and B in the LIF spectrum. Difference in transition frequencies of the observed DF peaks supports a two-conformer assignment. 


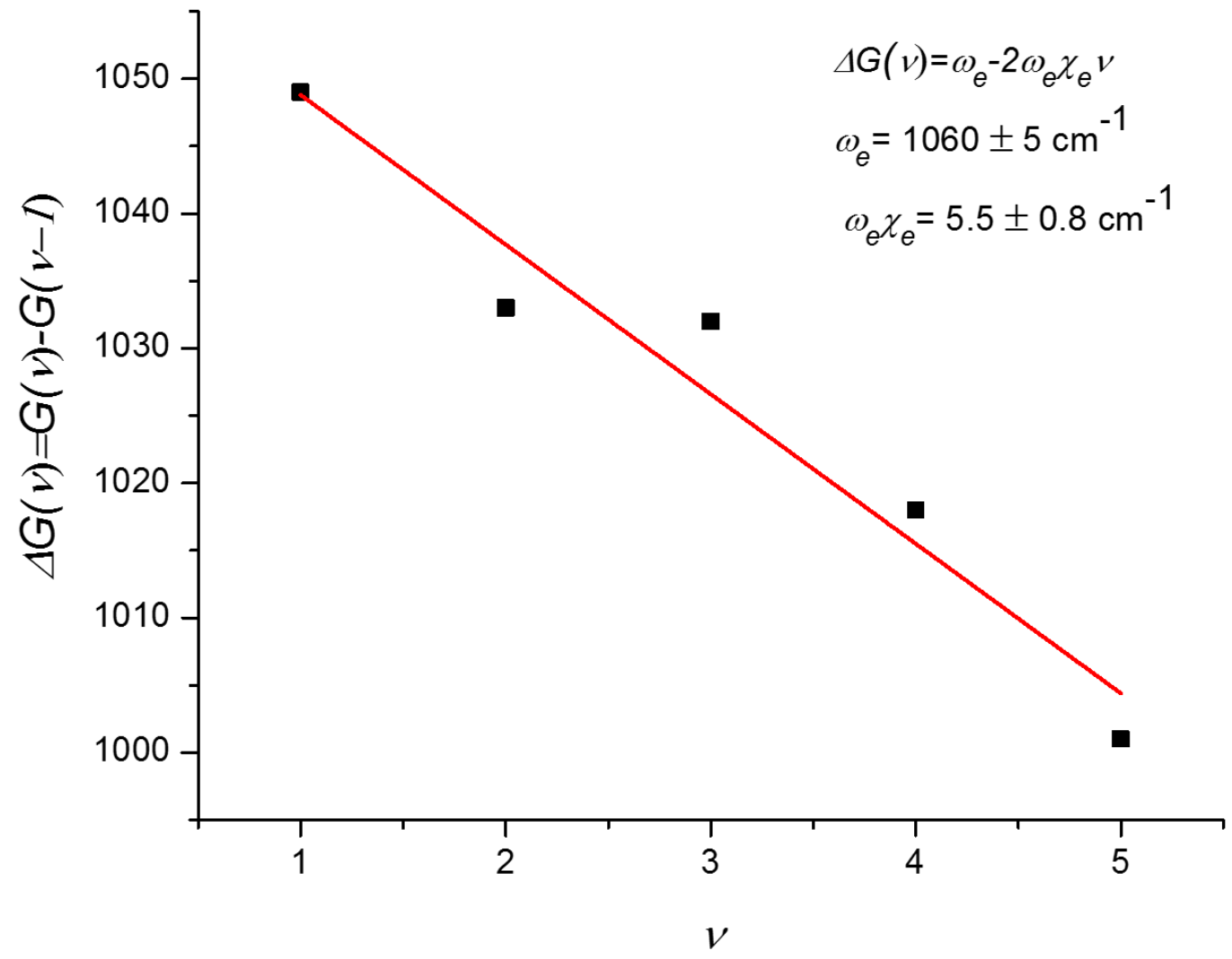

Figure 4.6. Determination of the harmonic frequency $\left(\omega_{e}\right)$ and the anharmonic parameter $\left(\omega_{e} \chi_{e}\right)$ of the $\tilde{X}$-state CO stretch of the $C_{l}$ conformer of isobutoxy. $G(v)$ is the vibrational energy of the $v^{\text {th }}$ member of the CO-stretch progression. 
a)

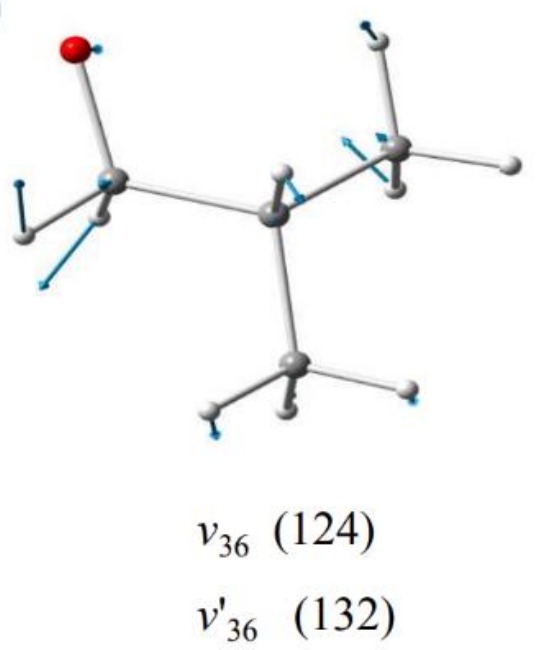

b)

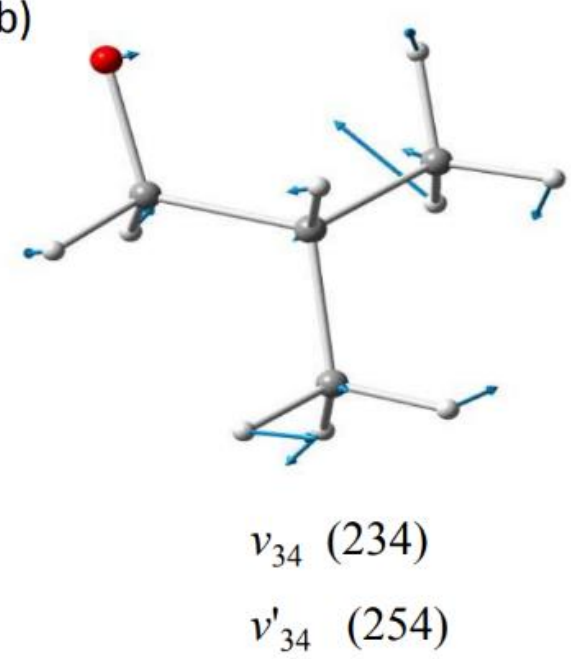

Figure 4.7. Vibrational modes of the $C_{1}$ conformer of isobutoxy. a) $-\mathrm{CH}_{2} \mathrm{O}$ wagging mode $\left(v_{36}\right)$, to which Bands C (in the LIF spectrum) and $\mathrm{c}$ (in the DF spectra) are assigned. b) in-phase $\mathrm{CH}_{3}$ torsional mode ( $\left.v_{34}\right)$, to which Bands D (in the LIF spectrum) and $d$ (in the DF spectra) are assigned. In parentheses are calculated vibrational frequencies in $\mathrm{cm}^{-1}$. Unprimed and primed labels are used to denote the $\tilde{X}$ - and $\tilde{B}$-state vibrational modes, respectively. 
a)

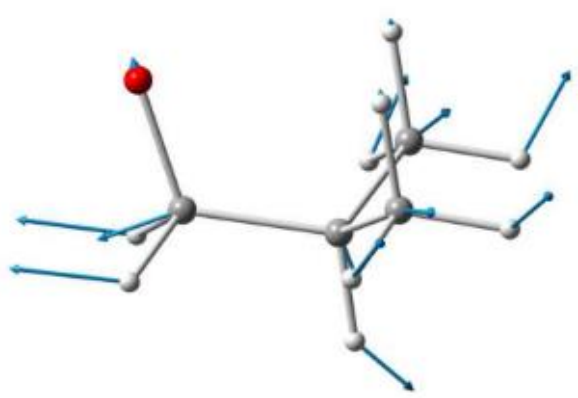

$v_{16}(783)$

c)

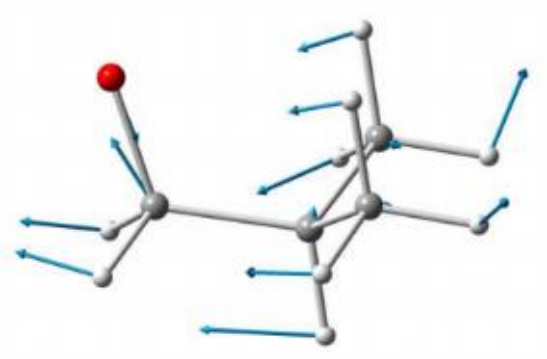

$v_{14}(1044)$ b)

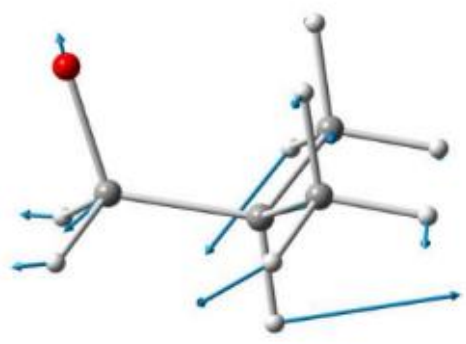

$v_{15}(943)$

d)

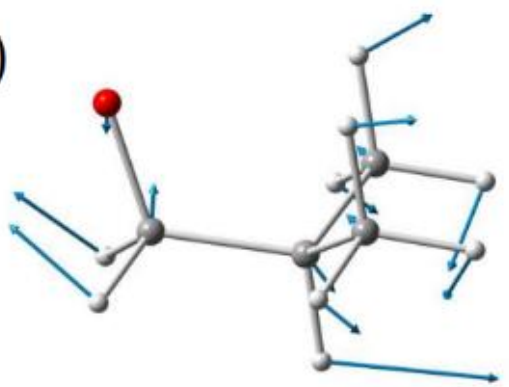

$v_{13}(1123)$

Figure 4.8. $\tilde{X}$-state vibrational modes of the $C_{\mathrm{s}}$ conformer of isobutoxy. a) $v_{16}$ (umbrella mode), b) $v_{15}$ (mixture of $\mathrm{CH}_{3}$ scissoring and $\mathrm{C}-\mathrm{C}$ stretch modes), c) $v_{14}$, and d) $v_{13} . v_{14}$ and $v_{13}$ are mixtures of $\mathrm{CO}$ stretch and the umbrella modes. 


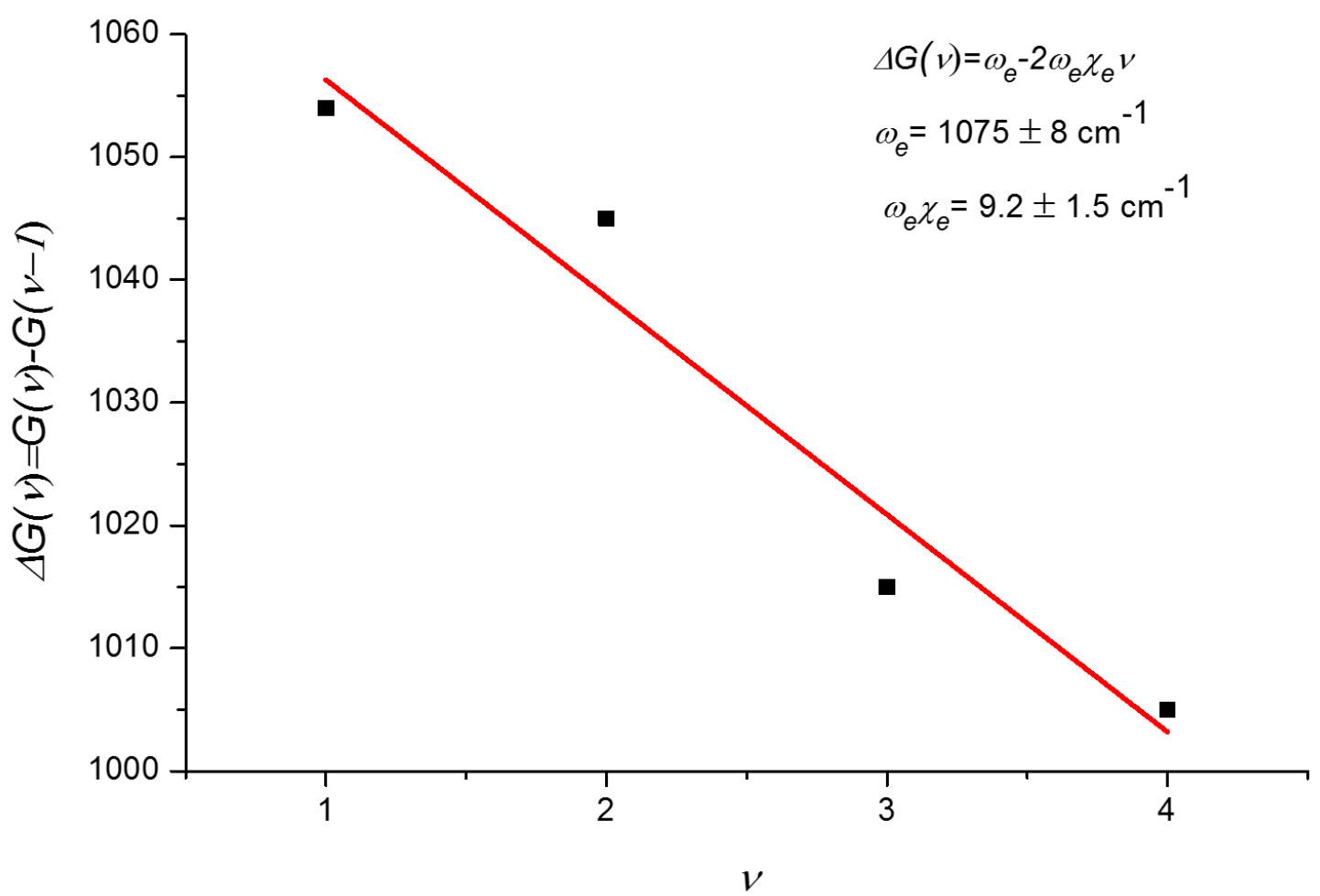

Figure 4.9. Determination of the harmonic frequency $\left(\omega_{e}\right)$ and the anharmonic parameter $\left(\omega_{e} \chi_{e}\right)$ of the $\tilde{X}$-state CO-stretch of the $\mathrm{G}_{1} \mathrm{~T}_{2}$ conformer of 2-methyl-1-butoxy. 
a)

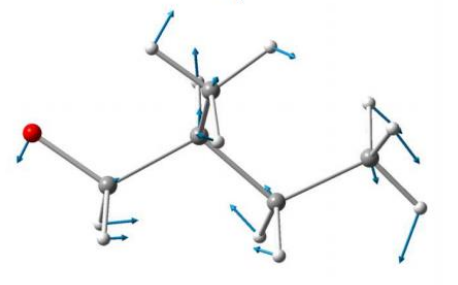

$v_{43}(210)$ b)

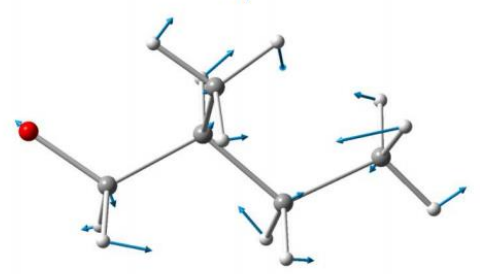

$v_{30}(1037)$ c)

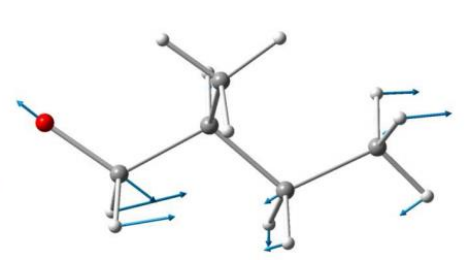

$v_{28}(1082)$

Figure 4.10. $\tilde{X}$-state vibrational modes of the $\mathrm{T}_{1} \mathrm{~T}_{2}$ conformer of 2-methyl-1-butoxy. a) $v_{43}$ (C-C-C bending mode), b) $v_{30}$, and c) $v_{28} . v_{30}$ and $v_{28}$ are mixtures of CO-stretch and the $\mathrm{C}-\mathrm{C}$ stretch modes.

a)

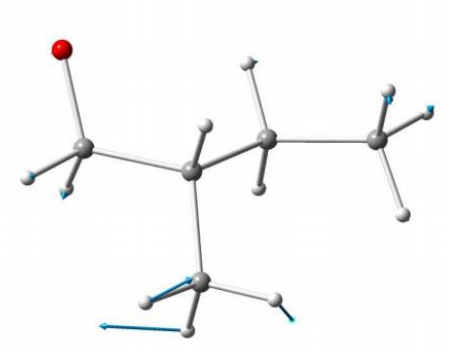

$v_{43}(206)$ b)

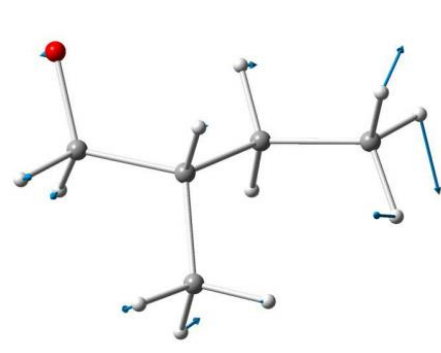

$v_{42}(217)$ c)

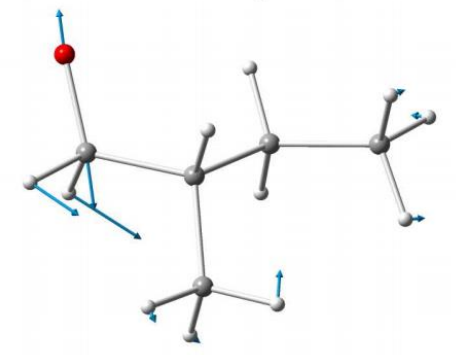

$v_{28}(1074)$

Figure $3.11 \tilde{X}$-state vibrational modes of the $\mathrm{G}_{1} \mathrm{~T}_{2}$ conformer of 2-methyl-1-butoxy radical. a) $v_{43}\left(\mathrm{C}_{3}\right.$-position methyl torsional mode), b) $v_{42}$ ( $\mathrm{C}_{4}$-position methyl torsional mode) and c) $v_{28}$ (CO-stretch mode). 


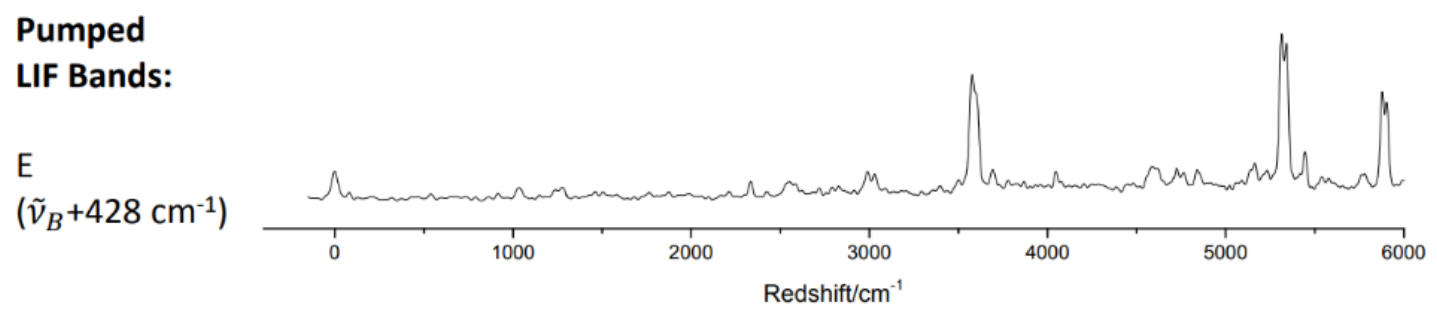

Figure 4.12. DF spectra of the isobutoxy radical by pumping Band $\mathrm{E}$ in its LIF spectrum, which suggests that the molecular carrier of Band $\mathrm{E}$ is formaldehyde. 


\section{CHAPTER V}

\section{LASER-INDUCED FLUORESCENCE AND DISPERSED FLUORESCENCE SPECTROSCOPY OF JET-COOLED ISOPENTOXY RADICALS}

\section{$5.1 \quad$ Introduction}

Alkoxy radicals are formed in high yields during the oxidation of hydrocarbons. They play key roles as reaction intermediates in low-temperature combustion, ${ }^{3,133}$ and the atmosphere, especially the troposphere. ${ }^{1,2}$ They have been studied by laser-induced

fluorescence (LIF), ${ }^{28,84,134}$ dispersed fluorescence (DF), ${ }^{34,93}$ and other laser-spectroscopy techniques. These spectroscopic investigations have furnished an understanding of the molecular structure and properties of alkoxy radicals and provided useful information for molecular dynamics and chemical reaction kinetic studies in which these important free radicals are involved. Such information includes origin frequencies, vibrational and rotational structure, and excited-state lifetimes. The atmospheric chemistry of large alkoxy radicals (with more than four carbons) is dominated by three main chemical reactions: reaction with $\mathrm{O}_{2}$, unimolecular dissociation via $\mathrm{C}-\mathrm{C}$ bond fission, and isomerization through $\mathrm{H}$-shift. These reactions can be highly sensitive to molecular structure. ${ }^{6}$ Conformation-specific spectroscopy investigations are, therefore, critical to the study of alkoxy radicals. 
Here we report LIF and DF spectra of one particular isomer of the pentoxy radicals, namely, 3-methyl-1-butoxy $\left[\left(\mathrm{CH}_{3}\right)_{2} \mathrm{CHCH}_{2} \mathrm{CH}_{2} \mathrm{O}\right]$, also known as the isopentoxy radical. Among all pentoxy isomers, isopentoxy is especially interesting in dynamics studies because of its $\beta \mathrm{C}-\mathrm{C}$ bond fission, which is affected strongly by its conformation.

Previously, the vibrationally resolved LIF spectrum of isopentoxy radicals produced in a supersonic jet expansion was reported by Liang et. al. ${ }^{29}$ However, no conformational or vibrational assignment was made because of the lack of clear vibrational progressions. In the present work, we report a new LIF measurement of the isopentoxy radical, in which more vibronic bands were observed owing to optimized experimental conditions and increased signal-to-noise ratio. In addition, DF spectra have been obtained by pumping different vibronic transitions in the LIF spectrum. Similarities between the DF spectra allow the differentiation of conformational carriers of the vibronic bands in the LIF spectrum. Both the conformational identification and the vibrational assignments are aided by simulations using newly calculated adiabatic electronic transition frequencies, vibrational frequencies of the ground and excited states, and the Franck-Condon factors (FCFs) of the vibronic transitions.

\subsection{Calculations}

\subsubsection{Potential energy surface (PES) scan and conformational search}

Conformers of isopentoxy are defined with two dihedral angles: $\Phi_{1}$ : $\mathrm{OC}_{1} \mathrm{C}_{2} \mathrm{C}_{3}$ and $\Phi_{2}$ : $\mathrm{C}_{1} \mathrm{C}_{2} \mathrm{C}_{3} \mathrm{H}$. Each dihedral angle can have three orientations in stable conformers: trans ( $\mathrm{T}$, $\left.\Phi \approx 180^{\circ}\right)$, counterclockwise gauche $\left(\mathrm{G}, \Phi \approx 60^{\circ}\right)$, and clockwise gauche $\left(\mathrm{G}^{\prime}, \Phi \approx-60^{\circ}\right)$ Internal rotation of the methyl groups does not introduce new stable conformations. 
Therefore, there are $3 \times 3=9$ conformers, 5 of which are optically distinguishable: TT, TG (TG'), GT (G'T), GG (G'G'), and $G^{\prime}\left(G^{\prime} G\right)$. (In parentheses are enantiomers.) Geometry optimization confirms that all five conformations are stable structures (see below). These five conformers are depicted in Figure 5.1.

\subsubsection{Quantum Chemical Calculations}

Significant computational work on isopentoxy has been done at the B3LYP/6-311++G(d,p) level of theory by Liang et al. ${ }^{29}$ No simulation of the $\tilde{B} \leftrightarrow \tilde{X}$ transitions was reported. In the present work, it has been found that accurate prediction of the $\tilde{B} \leftrightarrow \tilde{X}$ transition frequencies and, in most cases, satisfactory simulation of the LIF spectra of conformers can be achieved by including long-range corrections (see Section 5.3). The Coulomb-attenuating method CAM-B3LYP functional ${ }^{112}$ was adopted for the calculation of both ground and excited electronic states. All calculations presented here were done at the CAM-B3LYP/6$311+\mathrm{G}(\mathrm{d})$ (for the $\tilde{B}$-state) or time-dependent (TD-) CAM-B3LYP/6-311+G(d) for the $\tilde{B}$ state level of theory using the Gaussian 09 software package. ${ }^{114}$

Of all the geometric parameters, the $\tilde{B} \leftarrow \tilde{X}$ excitation affects the $\mathrm{CO}$ bond length, the $\mathrm{OC}_{1} \mathrm{C}_{2}$ angle, and the two dihedral angles $\left(\Phi_{1}\right.$ and $\left.\Phi_{2}\right)$ most significantly because the excitation corresponds to an electron promotion from the $p \sigma_{\mathrm{z}}$ to the $p \pi_{\mathrm{x}}$ or $p \pi_{\mathrm{y}}$ orbital of the oxygen atom. The optimized values of these geometric parameters for both the $\tilde{X}$ and $\tilde{B}$ states of all five conformers are summarized in Table 5.1. It is important to point out that the long-range Coulombic interaction between oxygen and the tertiary hydrogen leads to the large change of $\Phi_{1}$ and $\Phi_{2}$ upon the $\tilde{B} \leftarrow \tilde{X}$ excitation for the GG, GG' and GT $\left(\Phi_{1}=\mathrm{G}\right)$ 
conformers. For the TG conformer, the change of $\Phi_{1}$ and $\Phi_{2}$ is insignificant because of the larger distance between the oxygen and tertiary hydrogen atoms (see Figure 5.1).

Calculated relative energies of isopentoxy conformers, the adiabatic excitation frequencies of their $\tilde{B} \leftarrow \tilde{X}$ transitions, as well as the $\tilde{X}$ - and $\tilde{B}$-state CO stretch frequencies are listed in Table 5.2. Calculations show that three of the five conformers: GG', TG, and GG (referred to as Conformers 1, 2, and 4 in ref., ${ }^{29}$ respectively) are close in energy $(<0.3$ $\mathrm{kcal} / \mathrm{mol})$, while the other two are significantly higher in energy (>1 kcal/mol). Therefore, only the three lowest-energy conformers are expected to have detectable concentrations in the supersonic jet expansion. FCFs for $\tilde{B} \leftarrow \tilde{X}\left(v^{\prime \prime}=0\right)$ transitions of these three conformers were calculated using the ezSpectrum program. ${ }^{121}$ Due to the large difference in $\tilde{X}$ - and $\tilde{B}$ state geometries (Table 5.1), Duschinsky rotations were taken into account in the calculation of FCFs to better reproduce the experimental spectrum. ${ }^{122,123}$ Experimentally obtained LIF and DF spectra are simulated using calculated vibrational frequencies and FCFs (see below).

\section{$5.3 \quad$ Results}

\subsubsection{Overview of LIF and DF Spectra}

Figure 5.2a shows the vibrationally resolved LIF spectrum of the $\tilde{B} \leftarrow \tilde{X}$ transition of isopentoxy. It lacks the CO-stretch progressions that are typical in the LIF spectra of alkoxy radicals. Nevertheless, two vibrational progressions with different origins have been

observed in the low-frequency region. They are color-coded and labeled $\mathrm{A}_{0}, \mathrm{~A}_{1}, \mathrm{~A}_{2} \ldots$ and $\mathrm{B}_{0}, \mathrm{~B}_{1}, \mathrm{~B}_{2} \ldots$ in Figure 5.2a. Harmonic frequencies and anharmonic parameters of these 
two vibrational progressions were determined by fitting Birge-Sponer plots (Figure 5.5). For Progression A, $\omega_{e}=77.0 \pm 1.5 \mathrm{~cm}^{-1}$ and $\omega_{e} \chi_{e}=2.1 \pm 0.3 \mathrm{~cm}^{-1}$, while for Progression $\mathrm{B}, \omega_{e}=49.1 \pm 0.6 \mathrm{~cm}^{-1}$ and $\omega_{e} \chi_{e}=0.5 \pm 0.1 \mathrm{~cm}^{-1}$. Strong peaks in the higher-frequency region of the LIF spectrum are labeled Bands $\mathrm{C}$ through $\mathrm{L}$. They do not show any obvious pattern.

To help differentiate vibronic bands in the LIF spectrum by their molecular carriers, the DF spectra of isopentoxy were recorded by pumping different vibronic bands in the LIF spectrum. DF spectra obtained by pumping the A Bands do not contain any peaks other than the strong zero-redshift transition. Figure 5.6 shows an exemplary DF spectrum obtained by pumping Band $\mathrm{A}_{2}$.

DF spectra obtained by pumping the B Bands have similar vibronic structures, although they are shifted from each other (Figure 5.3). In all cases, a vibrational progression of $\sim 1025 \mathrm{~cm}^{-1}$ is discernable despite the broad linewidth of its members. The first two members of the progression are too weak to be detected. By extrapolation, the origin of the vibrational progression in the DF spectrum obtained by pumping each B Band can be determined. It is $409 \mathrm{~cm}^{-1}$ when Band $B_{0}$ is pumped.

DF spectra obtained by pumping Bands C-L (Figure 5.4) contain two strong vibrational progressions, both of which have frequency intervals of $\sim 1045 \mathrm{~cm}^{-1}$. When Band $\mathrm{C}$ or $\mathrm{L}$ is pumped, one of the progressions starts from zero red-shift, while the other starts with a weak peak at $263 \mathrm{~cm}^{-1}$ (labeled " $\mathrm{X}$ " in Figure 5.4a). DF spectra obtained by pumping Band D-K are similar, except that the two vibrational progressions are red-shifted. 
On the basis of the similarities and differences of the DF spectra, we attribute the three groups of LIF bands (the A Band, the B Bands, and Bands C-L) to three molecular carriers, namely, the three lowest-energy conformers of the isopentoxy radical. Bands $\mathrm{A}_{0}$ $\left(28598 \mathrm{~cm}^{-1}\right), \mathrm{B}_{0}\left(28821 \mathrm{~cm}^{-1}\right)$, and $\mathrm{C}\left(29058 \mathrm{~cm}^{-1}\right)$ are assigned to the origin bands, The cause of the red-shift in the DF spectra within each group has been discussed in our previous publications. ${ }^{35,93,96}$ In brief, the large change in the CO bond length upon the $\tilde{B}$ $\leftarrow \tilde{X}$ transition of alkoxy radicals leads to Duschinsky mixing ${ }^{122,123}$ between the COstretch mode and other vibrational modes. Consequently, when the free radical is excited to non-CO-stretch vibrational levels of the $\tilde{B}$-state, DF emission to combination levels of the $\mathrm{CO}$ stretch and the pumped vibrational mode are FC-favored. If the DF spectra are blue-shifted by the ground-state frequencies of the non-CO-stretch levels, the strong peaks will be lined up. (See Figures 5.8 and 5.9) The DF spectrum obtained by pumping Band L is not red-shifted to that obtained by pumping Band $\mathrm{C}$, an origin band. Therefore, Band $\mathrm{L}$ $\left(663 \mathrm{~cm}^{-1}\right.$ with respect to Band $\left.\mathrm{C}\right)$ is assigned to the $\mathrm{CO}$-stretch band.

\subsubsection{Simulation, Conformational Identification, and Vibrational Assignment of LIF} Spectrum

The calculated origin band frequencies of the three lowest-energy conformers of isopentoxy are $29650 \mathrm{~cm}^{-1}$ for $\mathrm{GG}^{\prime}, 29780 \mathrm{~cm}^{-1}$ for $\mathrm{GG}$, and $30036 \mathrm{~cm}^{-1}$ for TG. In comparison, transition frequencies for Band $\mathrm{A}_{0}, \mathrm{~B}_{0}$, and $\mathrm{C}$ are 28598, 28821, and 29058 $\mathrm{cm}^{-1}$, respectively. When a scaling factor of 0.967 is applied, the calculated origin-band frequencies reproduce the experimental ones quite well (see the $6^{\text {th }}$ and $7^{\text {th }}$ Columns of Table 5.2). The simulated LIF spectra of the GG', GG, and TG conformers are compared 
with the experimental LIF spectrum in Figures 5.2b-d, in which the calculated origin bands are shifted to match Bands $A_{0}, B_{0}$, and $C$, respectively.

The simulated LIF spectrum of the TG conformer (Figure 5.2b) reproduces the relative frequencies and intensities of most strong peaks in the higher-frequency region of the experimental LIF spectrum (Figure 5.2a) quite well. Comparing the relative frequencies of Bands C-L to the calculated vibrational frequencies of the $\tilde{B}$-state TG conformer, we are able to assign these vibronic bands, which confirms the assignment of Bands C-L to the TG conformer. The vibrational assignment is summarized in Table 5.3. The simulated LIF spectrum of the GG conformers contains a vibrational progression of $56 \mathrm{~cm}^{-1}$ in the lowfrequency region (labeled $\mathrm{B}_{0}, \mathrm{~B}_{1}, \mathrm{~B}_{2} \ldots$ in Figure 5.2c). These are transitions to the vibrational levels of the $\mathrm{C}_{1} \mathrm{C}_{2} \mathrm{C}_{3} \mathrm{H}$ torsion mode.

In comparison, the progression of B Bands in the experimental LIF spectrum has a harmonic frequency of $\omega_{\mathrm{e}}=49.1 \pm 0.6 \mathrm{~cm}^{-1}$. It is, therefore, reasonable to assign the B Bands to the GG conformer. The simulated LIF spectrum of the GG' conformer (Figure 5.2d) does not contain a progression of the lowest-frequency $\mathrm{C}_{1} \mathrm{C}_{2} \mathrm{C}_{3} \mathrm{H}$ torsion mode (calculated to be $\left.66 \mathrm{~cm}^{-1}\right)$. Instead, only the transition to its fundamental level has significant intensity (labeled $A_{1}$ in Figure 5.2d). Instead, transitions to several low-frequency vibrational modes and their combination bands are predicted.

Nevertheless, we assign the A Bands in the experimental LIF spectrum to this conformer based on two facts. First, the calculated (and scaled) origin transition of the GG' conformer matches the frequency of the $\mathrm{A}_{0}$ band. Second, the GG' conformer is the lowestenergy conformer and is expected to be present under jet-cooled conditions. Bands $A_{0}, B_{0}$, 
and $\mathrm{C}$ are the origin bands of the GG', TG, and GG conformers, respectively. Discrepancies between the simulated and experimental spectra will be discussed in Section 5.4.

\subsubsection{DF Spectra Obtained by Pumping the Origin Bands}

Simulation of DF spectra of isopentoxy was not attempted for two reasons: (i) severe broadening of transitions in the DF spectra, and (ii) the expected complexity of the vibronic structure of the quasi-degenerate $\tilde{X}$ - and $\tilde{A}$ - states. In a CAM-B3LYP calculation in which the HUMO and LUMO of the $\beta$ electron are swapped, ${ }^{132}$ the energy separation between the $\tilde{X}^{2} \mathrm{~A}$ " and the low-lying $\tilde{A}^{2} \mathrm{~A}$ ' states of the TT $\left(C_{\mathrm{s}}\right)$ conformer of isopentoxy was determined to be $208 \mathrm{~cm}^{-1} . \tilde{A}-\tilde{X}$ separations of other conformers, which have $C_{1}$ symmetry, cannot be calculated accurately but are expected to be comparable to that of the TT conformer. Due to the small $\tilde{A}-\tilde{X}$ separations, strong pseudo-Jahn-Teller and spin-orbit interactions between these two states are expected, which causes complicated vibronic structure. The broadening of the DF transitions may be attributed to both the complicated vibronic structure and the low isomerization barriers between conformers. (See Figure 5.10.)

Nevertheless, we may tentatively assign dominant features in the DF spectra according to quantum chemical calculations. In the DF spectrum obtained by pumping Band $\mathrm{B}_{0}$, the origin band of the GG conformer, the broad vibrational progression of $\sim 1025$ $\mathrm{cm}^{-1}$ is assigned to CO-stretch bands of the $\tilde{B} \rightarrow \tilde{A}$ transition of conformer GG. The CAMB3LYP calculation predicts several $\tilde{X}$-state modes that have CO-stretch character with frequencies of 1008, 1091, and $1111 \mathrm{~cm}^{-1}$. Similar CO-stretch modes are expected for the $\tilde{A}$ state. The existence of multiple vibrational modes with CO-stretch character may explain the unusually broad linewidths of features in the DF spectrum. The vibrational progression 
starts at $\sim 409 \mathrm{~cm}^{-1}$, which is attributed to the $\tilde{A}-\tilde{X}$ separation. The $\tilde{B} \rightarrow \tilde{X}$ transitions may be obscured by strong $\tilde{B} \rightarrow \tilde{A}$ transitions, although this cannot be confirmed from the present DF spectrum.

Similarly, the two $\sim 1045 \mathrm{~cm}^{-1}$ vibrational progressions in the DF spectrum obtained by pumping Band $\mathrm{C}$, the origin band of the TG conformer, are assigned to $\mathrm{CO}$ stretch bands of the $\tilde{B} \rightarrow \tilde{X}$ and $\tilde{B} \rightarrow \tilde{A}$ transitions of TG. This should be compared to the calculated $\tilde{X}$-state CO-stretch mode of $1096 \mathrm{~cm}^{-1}$, with another vibrational mode of 1037 $\mathrm{cm}^{-1}$ that has a CO-stretch character. The separation between the two vibrational progressions $\left(263 \mathrm{~cm}^{-1}\right)$ is attributed to the $\tilde{A}-\tilde{X}$ separation,

\subsection{Discussion}

Although quantum chemical calculations predict strong features in the LIF spectra of the GG' and GG conformers (Figure 5.2c and 5.2d), they are absent in the experimental spectrum (Figure 5.2a). Only two very weak low-frequency vibrational progressions (the A Bands and B Bands) are present in the low-frequency region. Similarly, for the TG conformer, although the calculation predicts a CO-stretch band (at $691 \mathrm{~cm}^{-1}$ relative to the origin band) that is significantly stronger than the origin band (Figure 5.2b), it is significantly weaker than the origin band in the experimental spectrum (Figure 5.2a). The discrepancy between the experimental and predicted transition intensities may be attributed to either $\tilde{B}$-state $\beta \mathrm{C}-\mathrm{C}$ fission or pre-dissociation of the $\tilde{\mathrm{B}}$ state via the $\mathrm{CO}$ bond breaking by highly excited electronic states. ${ }^{135}$ The latter mechanism is a more plausible explanation based on both calculations and experimental evidence. The barrier for ground-state $\beta \mathrm{C}-\mathrm{C}$ fission was calculated to be $12.9 \mathrm{kcal} / \mathrm{mol}\left(4512 \mathrm{~cm}^{-1}\right) \cdot{ }^{29}$ Experimentally, transitions of 
formaldehyde, one of the products of $\beta \mathrm{C}-\mathrm{C}$ fission was observed in our LIF experiment (see Figure 5.11), which confirms the low barrier. Although a TD-CAM-B3LYP calculation of the barrier for $\tilde{B}$-state $\beta \mathrm{C}-\mathrm{C}$ fission was not successful, the barrier is expected to be lower than the ground state because in the $\tilde{B}$-state the half-filled $2 p \sigma$ orbital of the $\mathrm{CO}$ bond further weakens the adjacent $\mathrm{C}-\mathrm{C}$ bond.

The two low-frequency vibrational progressions in the experimental LIF spectrum are assigned to GG and GG' conformers. They correspond to the lowest-frequency vibrational modes of these two conformers, which involve the torsion of the $\Phi_{2}\left(\mathrm{C}_{1} \mathrm{C}_{2} \mathrm{C}_{3} \mathrm{H}\right)$ dihedral angle. For the GG conformer, the CAM-B3LYP calculation predicts a very large change of $\Phi_{2}$ upon $\tilde{B} \leftarrow \tilde{X}$ excitation (from $56^{\circ}$ to $33^{\circ}$ ). Hence, the simulated LIF spectrum of this conformer contains a long vibrational progression of this mode. For the GG' conformer, the change of $\Phi_{2}$ (from $-52^{\circ}$ to $-65^{\circ}$ ), although still significant, is much smaller than for the GG conformer. The predicted LIF spectrum of this conformer, therefore, does not contain a low-frequency vibrational progression, whereas the experimental LIF spectrum does. Therefore, we suspect that the CAM-B3LYP calculation underestimates the $\Phi_{2}$ dihedral angle of GG' in its $\tilde{B}$ state. Calculations using other density-functional methods with long-range corrections, including wB97XD, have been employed ${ }^{136}$ to optimize the $\tilde{B}$-state geometries of isopentoxy conformers. The results are close to those using CAM-B3LYP. Further computational investigation using other methods is therefore necessary to reproduce the experimentally obtained LIF spectrum. 


\subsection{Conclusions}

We have observed $\tilde{B}-\tilde{X}$ LIF and DF spectra of jet-cooled isopentoxy radicals. Of all five conformers (GG', TG, GG, TT, and GT), the three lowest-energy ones (GG', TG, and GG) are identified as spectral carriers based on vibronic simulations. LIF spectra of GG' and GG conformers contain low-frequency vibrational progressions due to large changes of the $\mathrm{C}_{1} \mathrm{C}_{2} \mathrm{C}_{3} \mathrm{H}$ dihedral angle between the $\tilde{B}$ - and $\tilde{X}$-states. Most experimentally observed LIF transitions are reproduced in simulations using electronic transition frequencies, vibrational frequencies, and Franck-Condon factors calculated at the CAM-B3LYP/6$311+\mathrm{G}(\mathrm{d})$ level of theory. The absence of strong transitions to the CO-stretch levels is attributed to low-barriers for $\beta \mathrm{C}-\mathrm{C}$ fission or predissociation in the $\tilde{B}$-state. DF spectra of the GG and TG conformers are dominated by strong vibrational progressions of the $\mathrm{CO}$ stretch mode or its combination with other vibrational modes due to Duschinsky mixing.

The $\tilde{A}-\tilde{X}$ separations of the GG and TG conformers have been determined to be 409 and $263 \mathrm{~cm}^{-1}$, respectively. 
Table 5.1. Geometric parameters of conformers of the isopentoxy radical in the $\tilde{X}$ - and $\tilde{B}$ states calculated at the CAM-B3LYP/6-311+G(d) level of theory.

\begin{tabular}{ccccccccc}
\hline \multirow{2}{*}{ conformer } & \multicolumn{2}{c}{$\mathrm{C}-\mathrm{O}(\AA)$} & \multicolumn{2}{c}{$\begin{array}{c}\Phi_{1} \mathrm{OC}_{1} \mathrm{C}_{2} \mathrm{C}_{3} \\
\text { (deg.) }\end{array}$} & \multicolumn{2}{c}{$\begin{array}{c}\Phi_{2} \mathrm{C}_{1} \mathrm{C}_{2} \mathrm{C}_{3} \mathrm{H} \\
\text { (deg.) }\end{array}$} \\
\cline { 2 - 9 } & $\tilde{\mathrm{X}}$ & $\tilde{\mathrm{B}}$ & $\tilde{\mathrm{X}}$ & $\tilde{\mathrm{B}}$ & $\tilde{\mathrm{X}}$ & $\tilde{\mathrm{B}}$ & $\tilde{\mathrm{X}}$ & $\tilde{\mathrm{B}}$ \\
\hline GG' & 1.36 & 1.58 & 116 & 107 & 61 & 57 & -52 & -65 \\
TG & 1.36 & 1.59 & 115 & 107 & 177 & 175 & 56 & 57 \\
GG & 1.36 & 1.59 & 116 & 109 & 75 & 65 & 56 & 33 \\
$\mathrm{TT}$ & 1.36 & 1.6 & 115 & 107 & 178 & 180 & 179 & 180 \\
$\mathrm{GT}$ & 1.36 & 1.59 & 115 & 109 & 72 & 63 & 179 & 158 \\
\hline
\end{tabular}




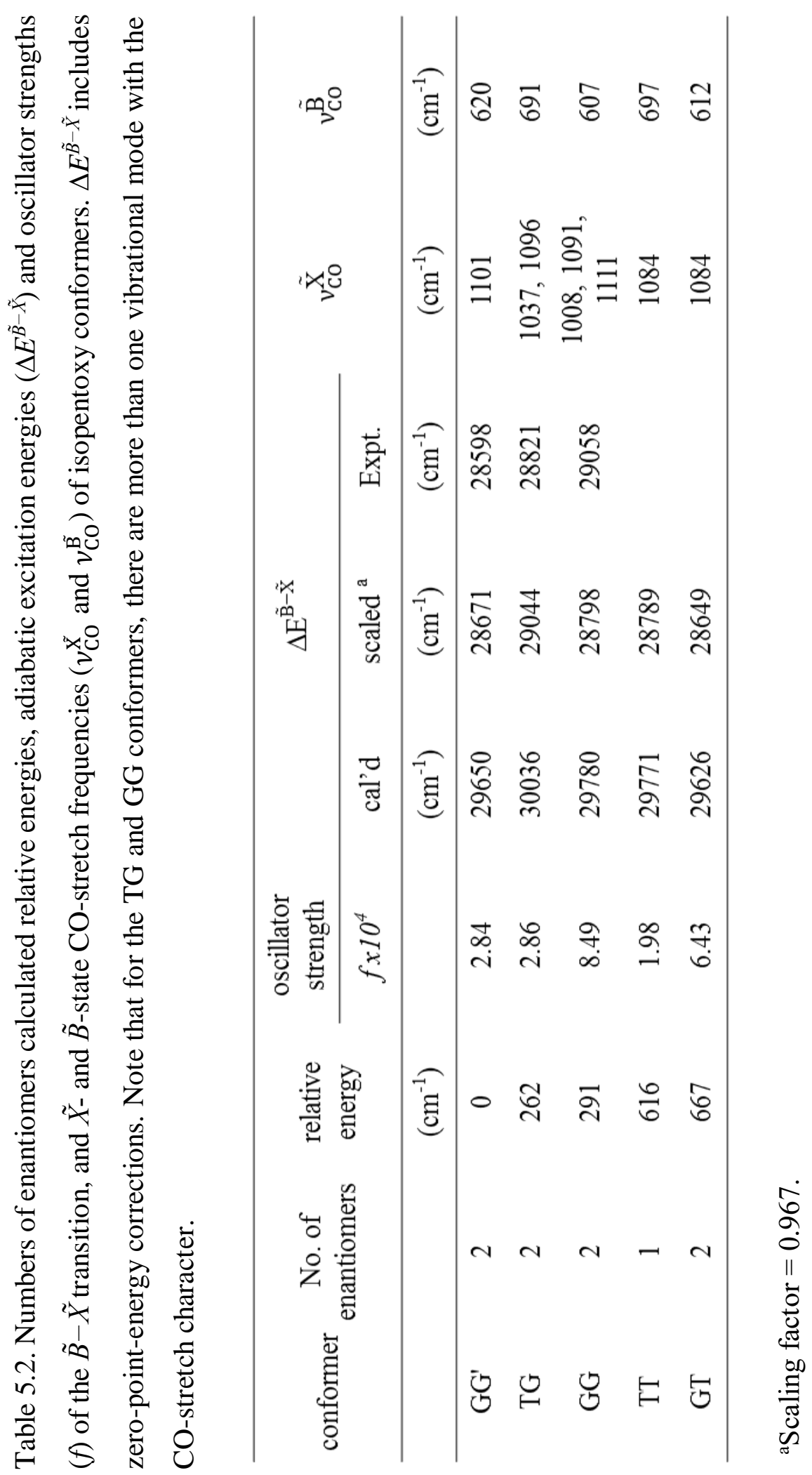


Table 5.3. Experimental and calculated vibrational frequencies $\left(\right.$ in $\mathrm{cm}^{-1}$ ) and assignment of vibronic bands in the LIF spectra (Figure 4.2a).

\begin{tabular}{|c|c|c|c|c|}
\hline Conformer & Band & Expt. $^{\mathrm{a}}$ & $\mathrm{Cal}^{\mathrm{b}}$ & Assignment $^{\mathrm{c}}$ \\
\hline \multirow{5}{*}{ GG' } & $\mathrm{A}_{0}$ & 0 & 0 & $v_{00} \tilde{\mathrm{B}} \leftarrow \tilde{\mathrm{X}}$ \\
\hline & $\mathrm{A}_{1}$ & 73 & 66 & $v_{45}$ \\
\hline & $\mathrm{A}_{2}$ & 142 & 128 & $v_{44}$ \\
\hline & $\mathrm{A}_{3}$ & 205 & 216 & $v_{43}$ \\
\hline & $\mathrm{A}_{4}$ & 266 & 286 & $v_{41}$ \\
\hline \multirow{6}{*}{ GG } & $\mathrm{B}_{0}$ & 0 & 0 & $v_{00}^{\tilde{B}} \leftarrow \tilde{X}$ \\
\hline & $\mathrm{B}_{1}$ & 48 & 56 & $v_{45}$ \\
\hline & $\mathrm{B}_{2}$ & 95 & 113 & $2 v_{45}$ \\
\hline & B3 & 142 & 169 & $3 v_{45}$ \\
\hline & $\mathrm{B}_{4}$ & 188 & 225 & $4 v_{45}$ \\
\hline & $\mathrm{B}_{5}$ & 232 & 275 & $v_{45}+v_{44}$ \\
\hline \multirow{14}{*}{$\mathrm{TG}$} & $\mathrm{C}$ & 0 & 0 & $v_{00}^{\tilde{B}} \leftarrow \tilde{\mathrm{X}}$ \\
\hline & $\mathrm{D}$ & 68 & & \\
\hline & $\mathrm{E}$ & 133 & 105 & $v_{44}$ \\
\hline & & 167 & 161 & $2 v_{45}$ \\
\hline & $\mathrm{F}$ & 177 & 179 & $v_{43}$ \\
\hline & & 205 & & \\
\hline & G & 224 & 235 & $v_{42}$ \\
\hline & $\mathrm{H}$ & 310 & 320 & $v_{40}$ \\
\hline & I & 354 & 357 & $2 v_{43}$ \\
\hline & & 411 & 413 & $v_{43}+v_{42}$ \\
\hline & $\mathrm{J}$ & 417 & 426 & $v_{38}$ \\
\hline & & 487 & 499 & $v_{43}+v_{40}$ \\
\hline & $\mathrm{K}$ & 508 & & \\
\hline & $\mathrm{L}$ & 663 & 691 & $v_{36}$ \\
\hline
\end{tabular}

a Observed band maximum $\left(\mathrm{cm}^{-1}\right)$ relative to the origin band $\left(v_{00}^{\tilde{\mathrm{B}} \leftarrow \tilde{\mathrm{X}}}\right)$ at $28598 \mathrm{~cm}^{-1}\left(\mathrm{~A}_{0}\right)$, $28821 \mathrm{~cm}^{-1}\left(\mathrm{~B}_{0}\right)$, and $29058 \mathrm{~cm}^{-1}(\mathrm{C})$ for the $\mathrm{GG}^{\prime}, \mathrm{GG}$, and TG conformer, respectively. ${ }^{\mathrm{b}}$ Unscaled $\tilde{B}$-state vibrational frequencies calculated at the CAM-B3LYP/6-311+G(d) level of theory.

${ }^{c}$ Vibrational modes are numbered following the Herzberg convention. $v_{45}: \mathrm{C}_{1} \mathrm{C}_{2} \mathrm{C}_{3} \mathrm{H}$ torsion. $v_{36}$ of TG: CO stretch. 

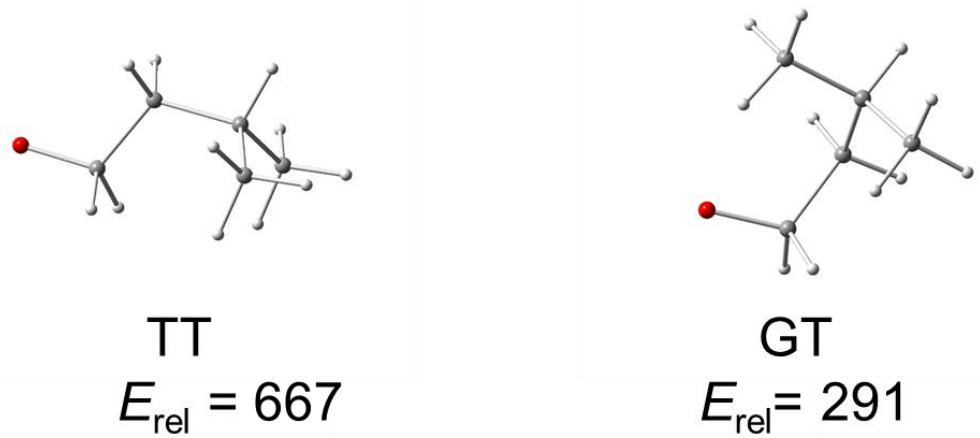

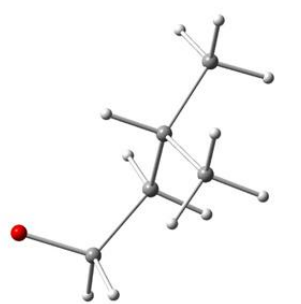

GG'

$$
E_{\text {rel }}=0
$$

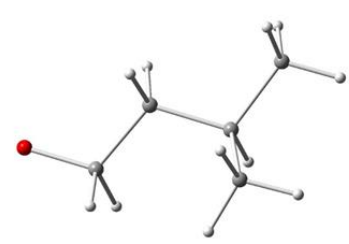

TG

$E_{\text {rel }}=262$

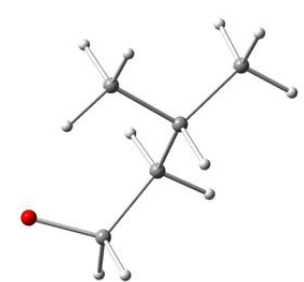

GG

$E_{\text {rel }}=291$

Figure 5.1. The five conformers of isopentoxy and their relative energies $\left(\mathrm{cm}^{-1}\right)$. 


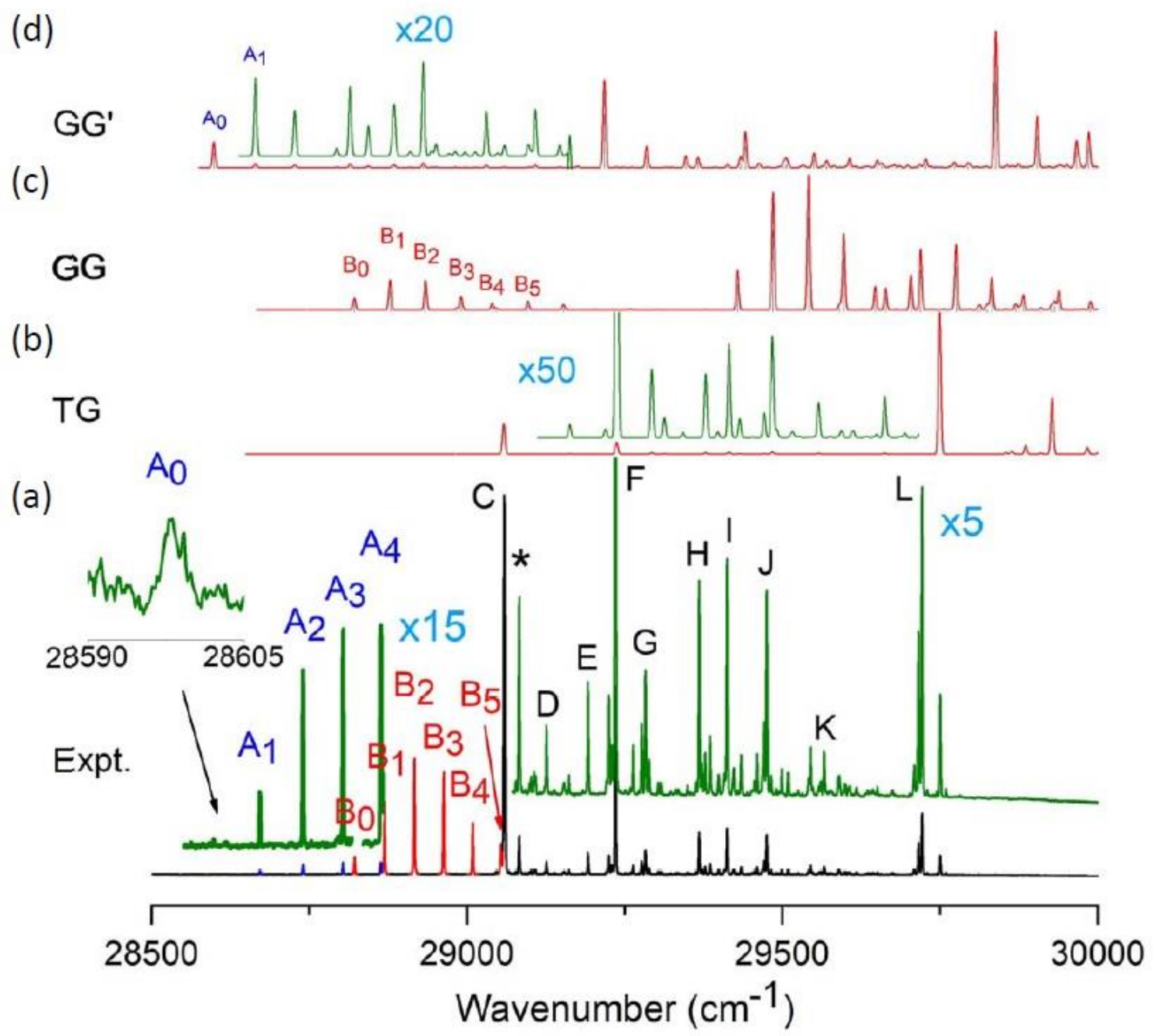

Figure 5.2. (a) Vibrationally resolved LIF spectrum of isopentoxy. (b-d) Simulated LIF spectra of the GG' (b), GG (c) and TG (d) conformers. The asterisked band is due to $\mathrm{CH}_{2} \mathrm{O}$. 


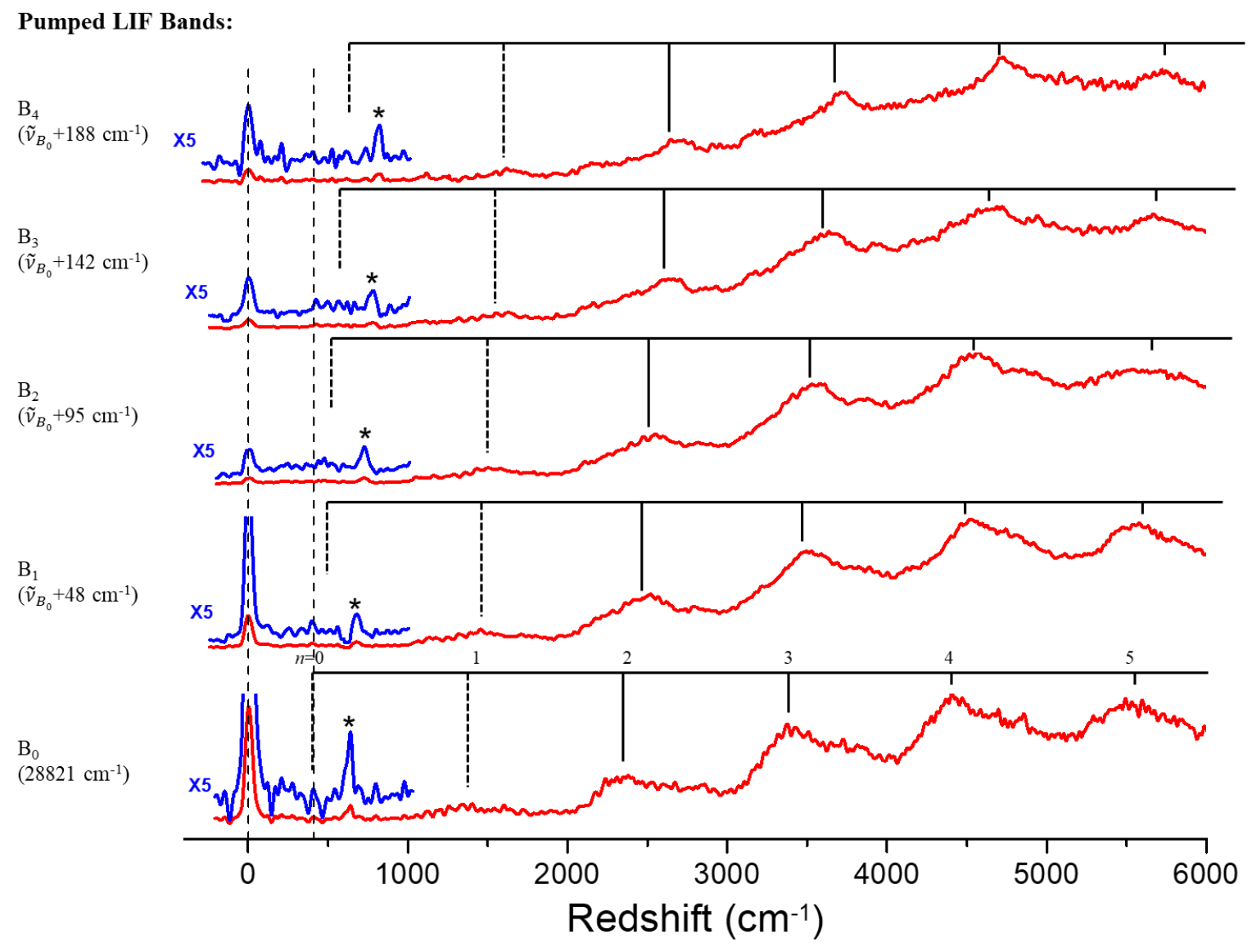

Figure 5.3. DF spectra of the isopentoxy radical obtained by pumping LIF bands $\mathrm{B}_{0}-\mathrm{B}_{4}$. Numbers in parentheses after the pumped LIF bands are relative wavenumbers with respect to the origin band $\left(B\right.$ and $\mathrm{B}_{0}$ ). The asterisked bands are due to photolysis laser scattering. 
Pumped LIF Bands:

$\mathrm{L}$

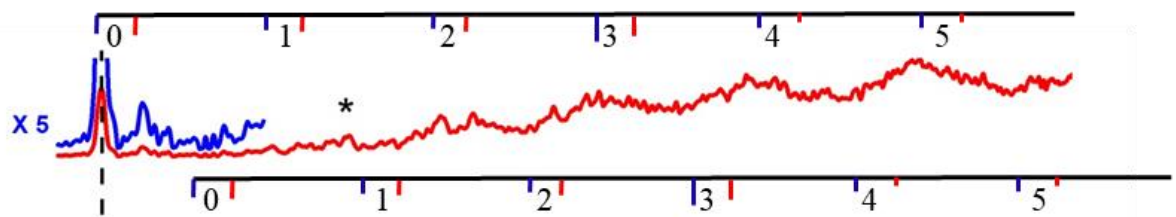

$\mathrm{K}$

$\left(\tilde{v}_{C}+508 \mathrm{~cm}^{-1}\right)$

$\mathrm{J}$

$\left(\tilde{v}_{C}+417 \mathrm{~cm}^{-1}\right)$

I

$\left(\tilde{v}_{C}+354 \mathrm{~cm}^{-1}\right)$

$\mathrm{H}$

$\mathrm{H}$
$\left(\tilde{v}_{C}+310 \mathrm{~cm}^{-1}\right)$
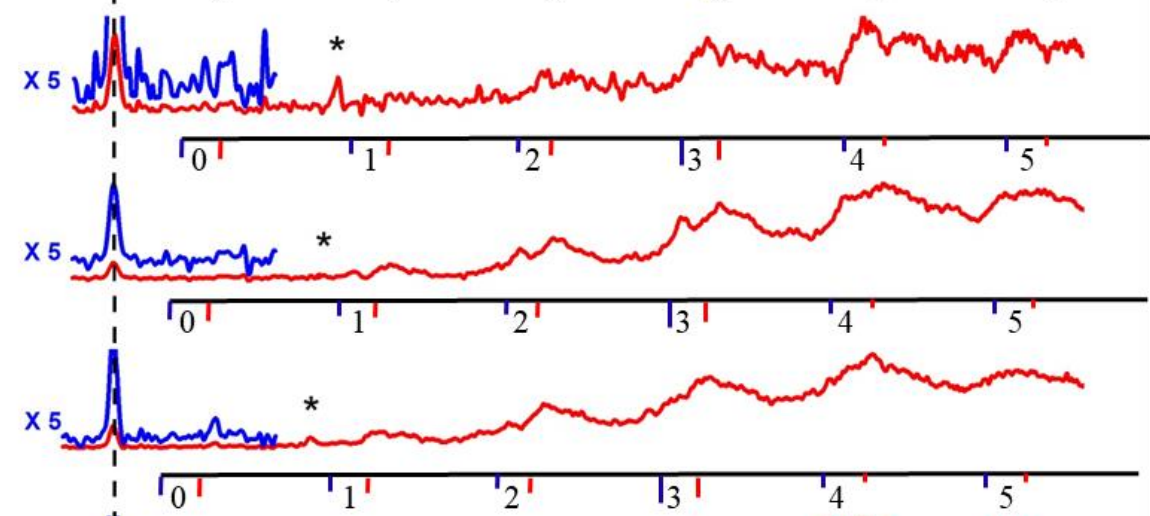

$\mathrm{G}$

$\left(\tilde{v}_{C}+224 \mathrm{~cm}^{-1}\right)$
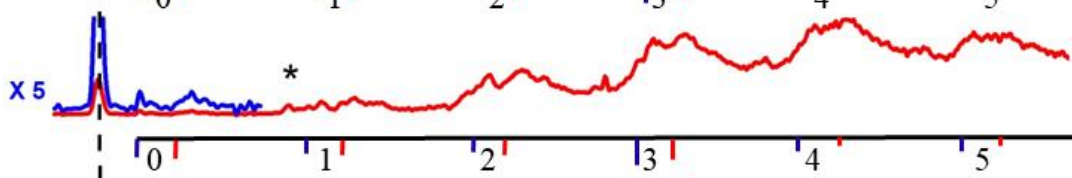

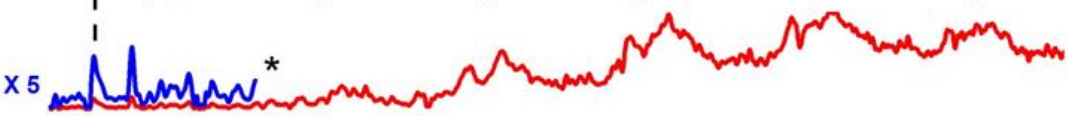

$\mathrm{F}$

$\left(\tilde{v}_{C}+177 \mathrm{~cm}^{-1}\right)$

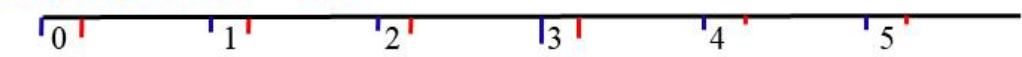

$\mathrm{E}$

$\left(\tilde{v}_{C}+133 \mathrm{~cm}^{-1}\right)$
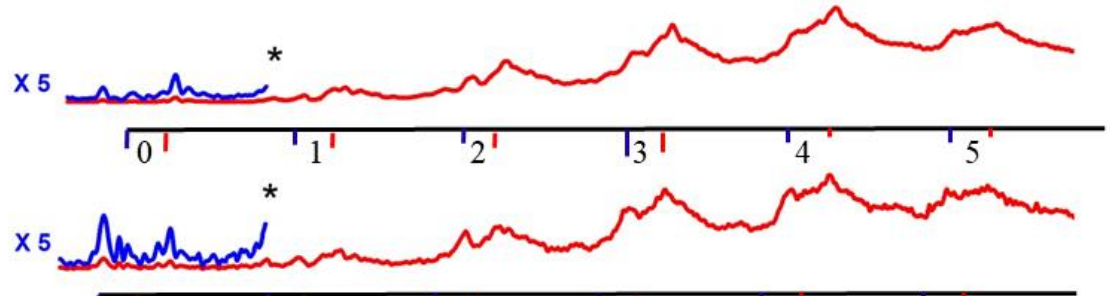

$\mathrm{C}$

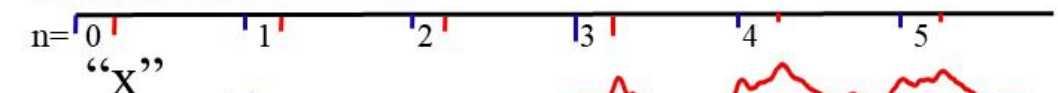

$\left(29058 \mathrm{~cm}^{-1}\right)$

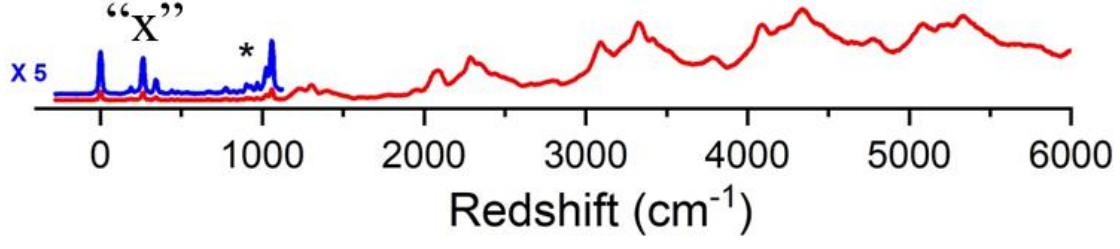

Figure 5.4. DF spectra of the isopentoxy radical obtained by pumping LIF bands C-L. Numbers in parentheses after the pumped LIF bands are relative wavenumbers with respect to the origin band (Band $\mathrm{C}$ ). The asterisked bands are due to photolysis laser scattering. 


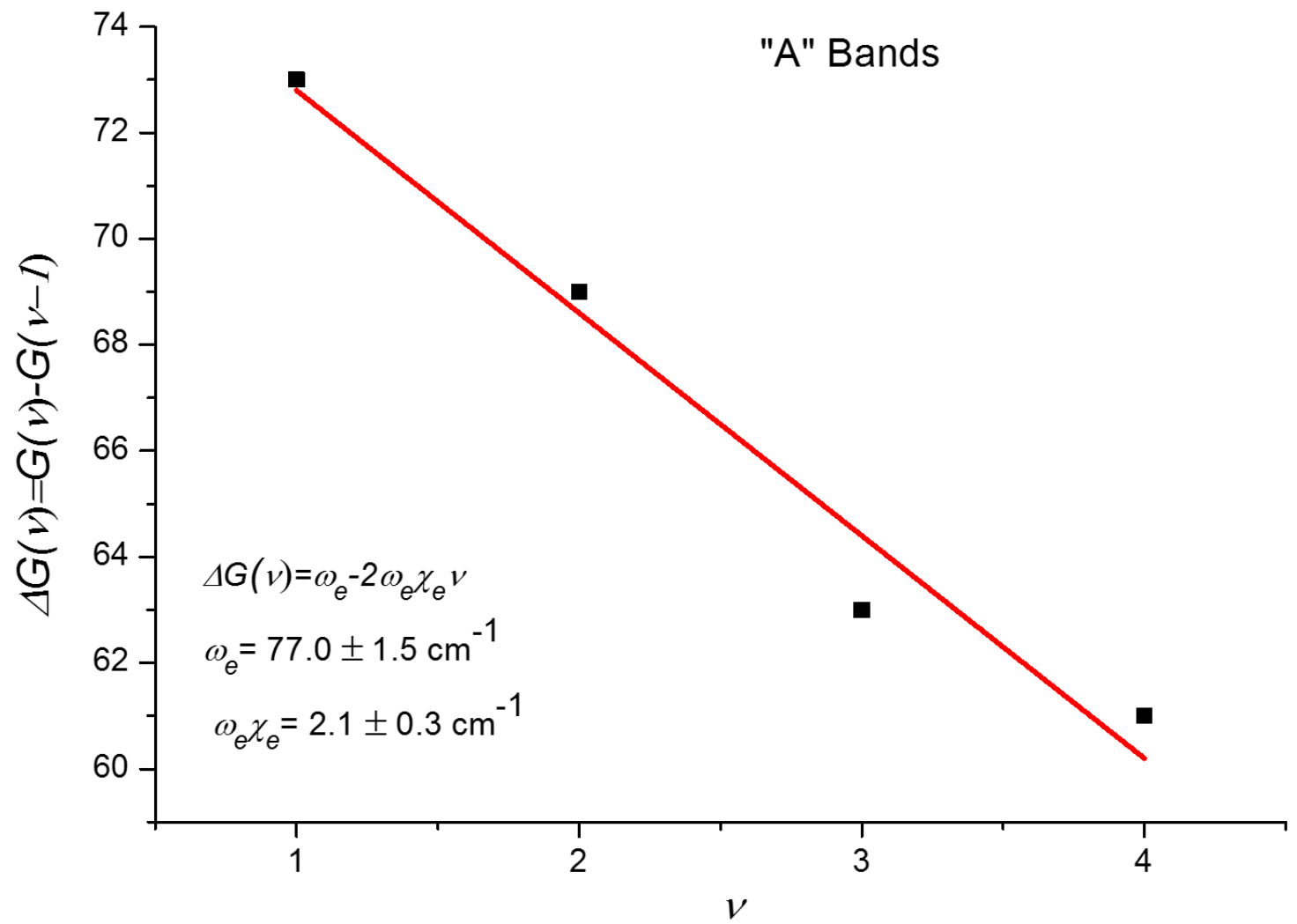

Figure 5.5. Birge-Sponer plot of the "A" Bands in the LIF spectrum of isopentoxy. 


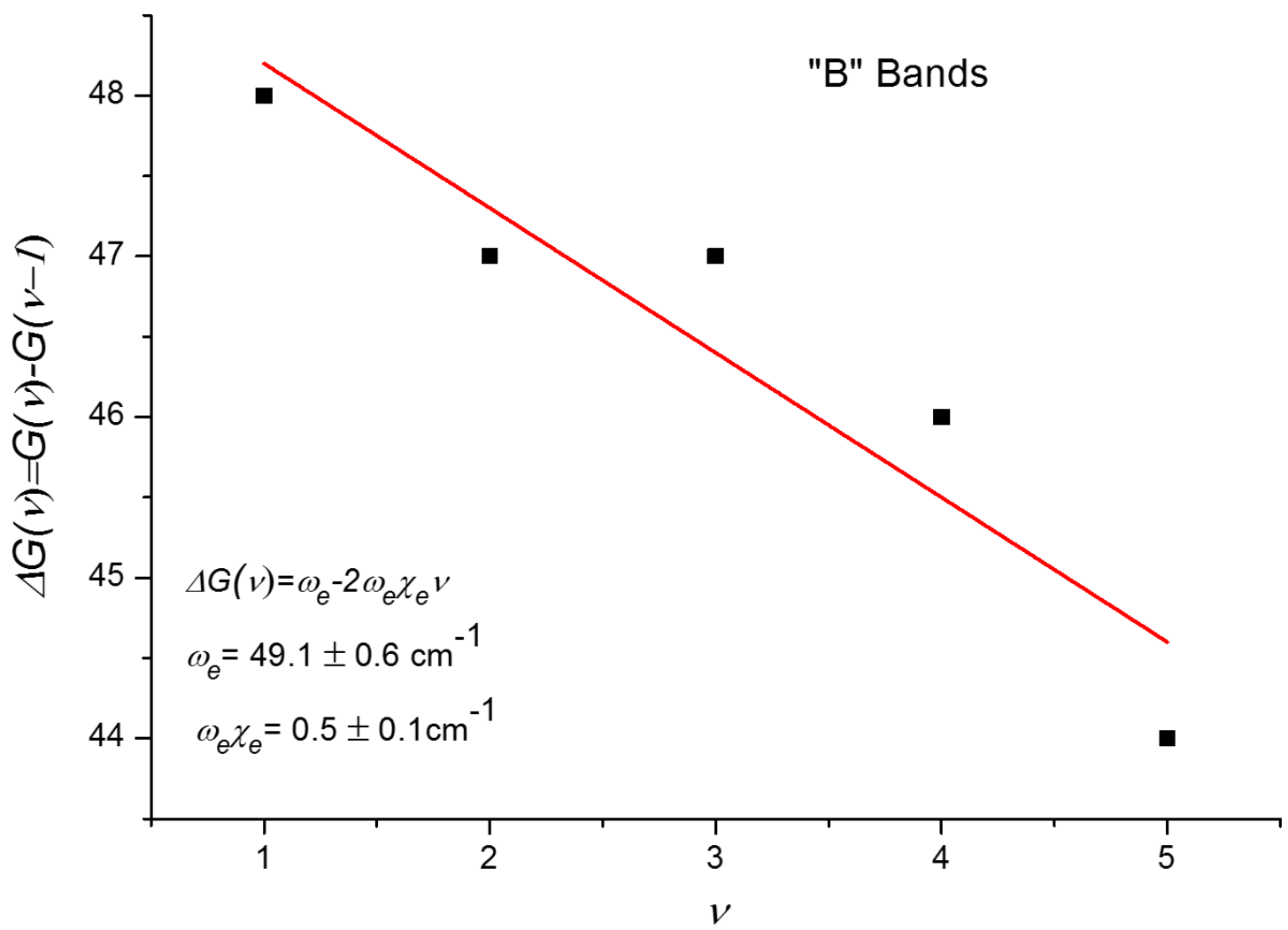

Figure 5.6. Birge-Sponer plot of the "B" Bands in the LIF spectrum of isopentoxy. 


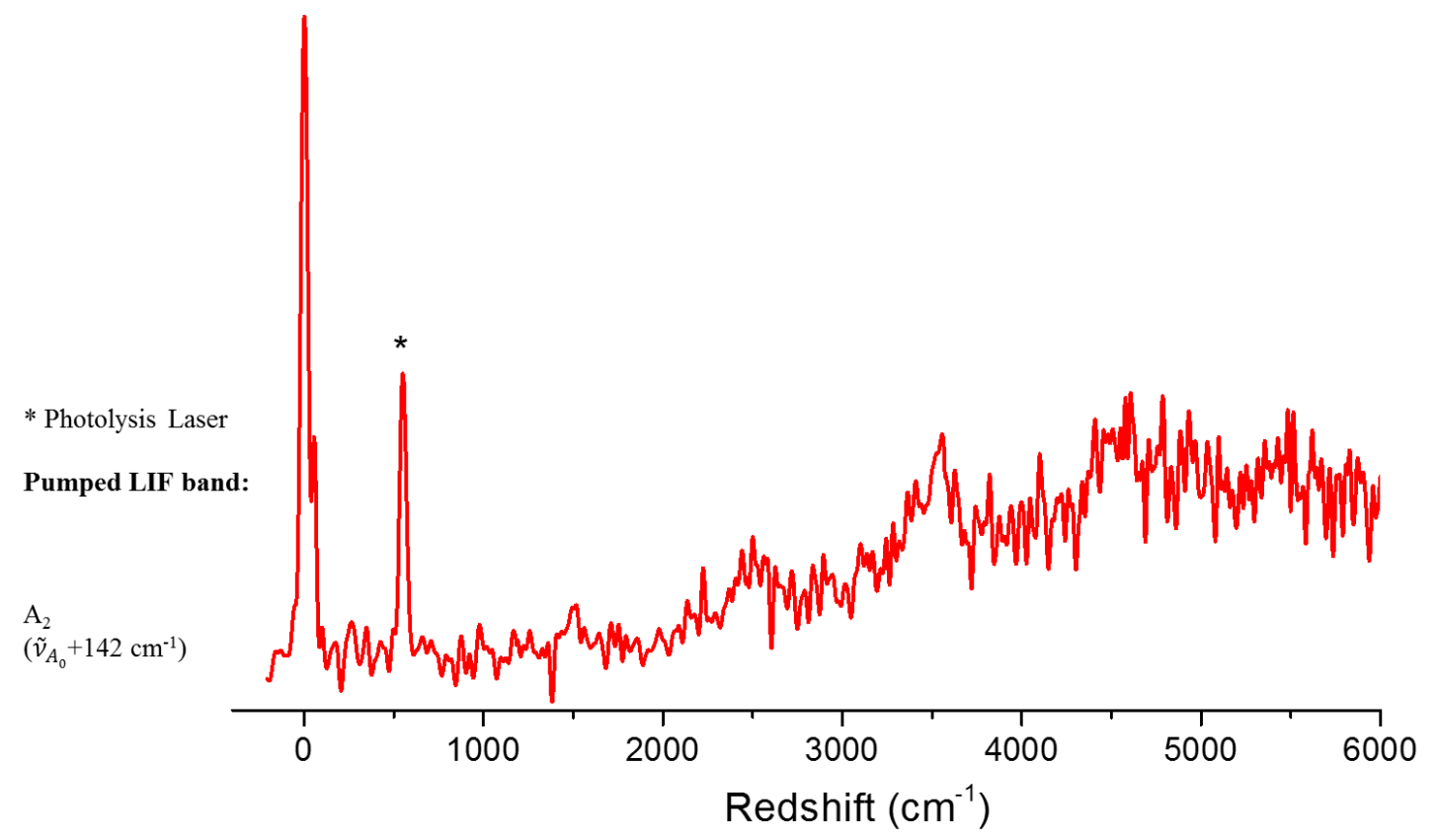

Figure 5.7. DF spectrum of the isopentoxy radical obtained by pumping LIF bands $A_{2}$. Numbers in parentheses after the pumped LIF bands are relative wavenumbers with respect to the origin band (Band $A_{0}$ ). The asterisked band is due to photolysis laser scattering. 


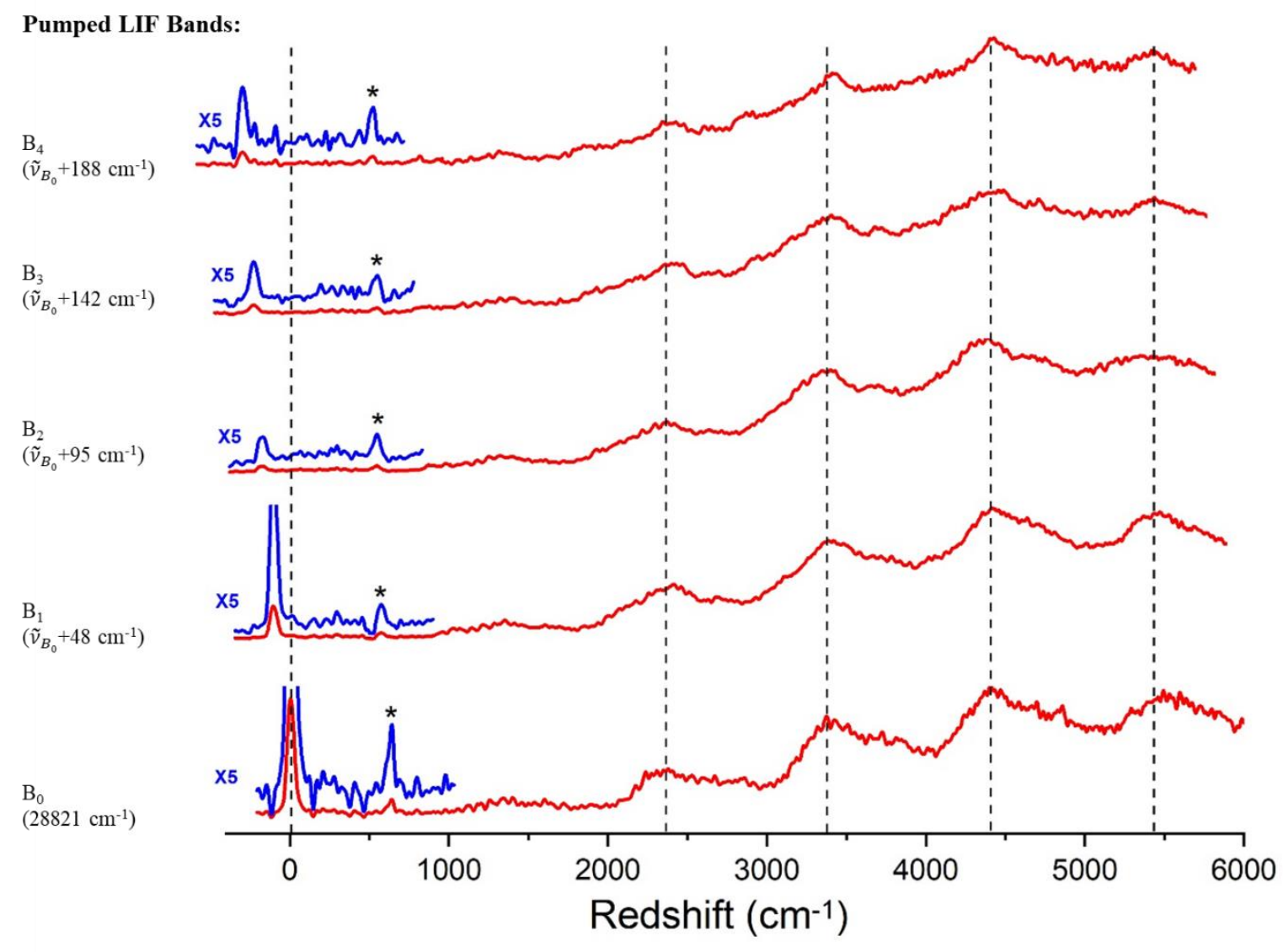

Figure 5.8. DF spectra of the isopentoxy radical obtained by pumping LIF bands $\mathrm{B}_{0}-\mathrm{B}_{4}$. The DF spectra are blue-shifted by ground-state vibrational frequencies of the pumped modes. Numbers in parentheses after the pumped LIF bands are relative wavenumbers with respect to the origin band $\left(\mathrm{Band}_{0}\right)$. The asterisked band is due to photolysis laser scattering. 


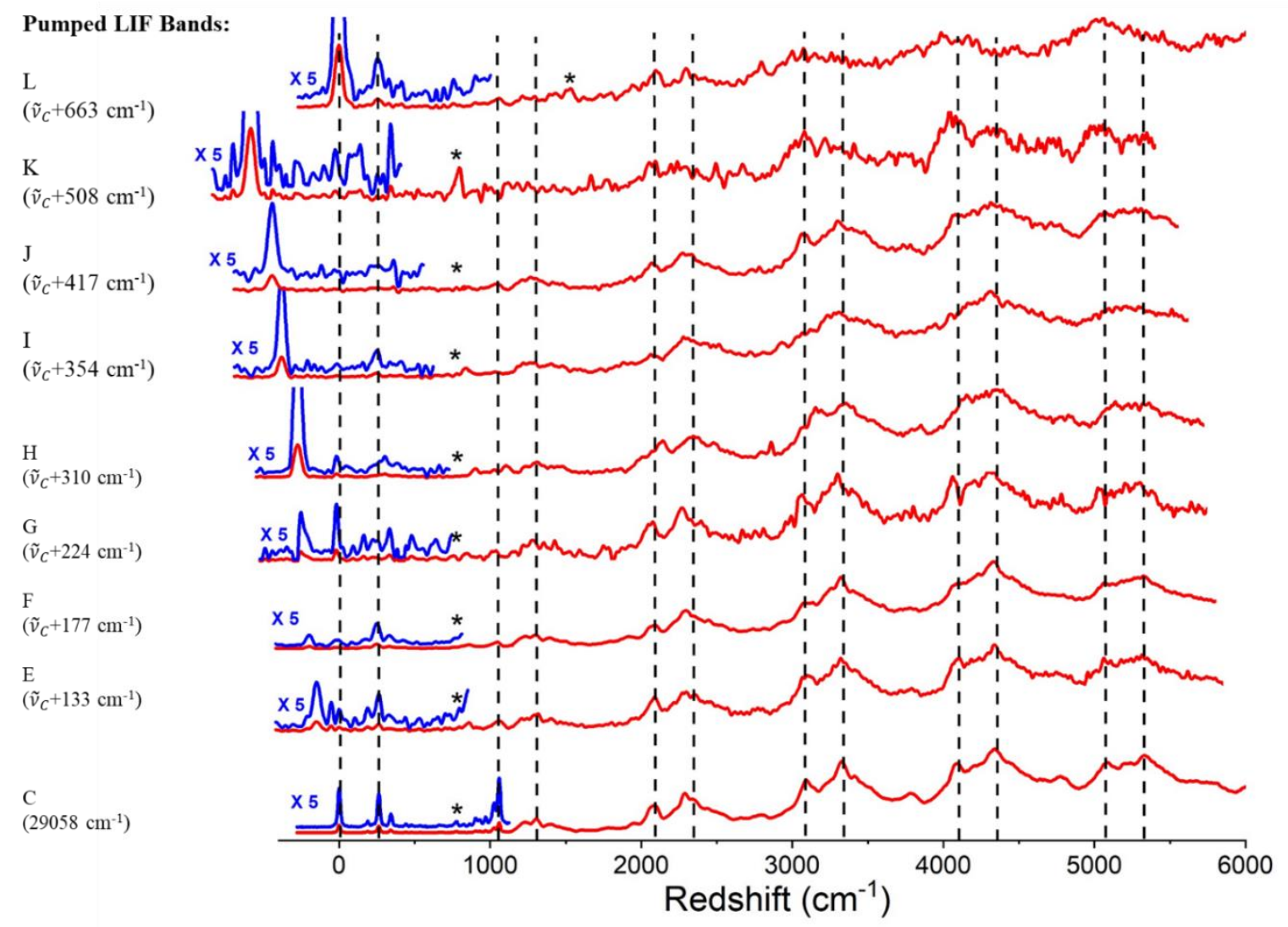

Figure 5.9. DF spectra of the isopentoxy radical obtained by pumping LIF bands C-L. The DF spectra except that obtained by pumping the CO stretch band (Band L) are blueshifted by ground-state vibrational frequencies of the pumped modes. Numbers in parentheses after the pumped LIF bands are relative wavenumbers with respect to the origin band (Band C). The asterisked band is due to photolysis laser scattering. 


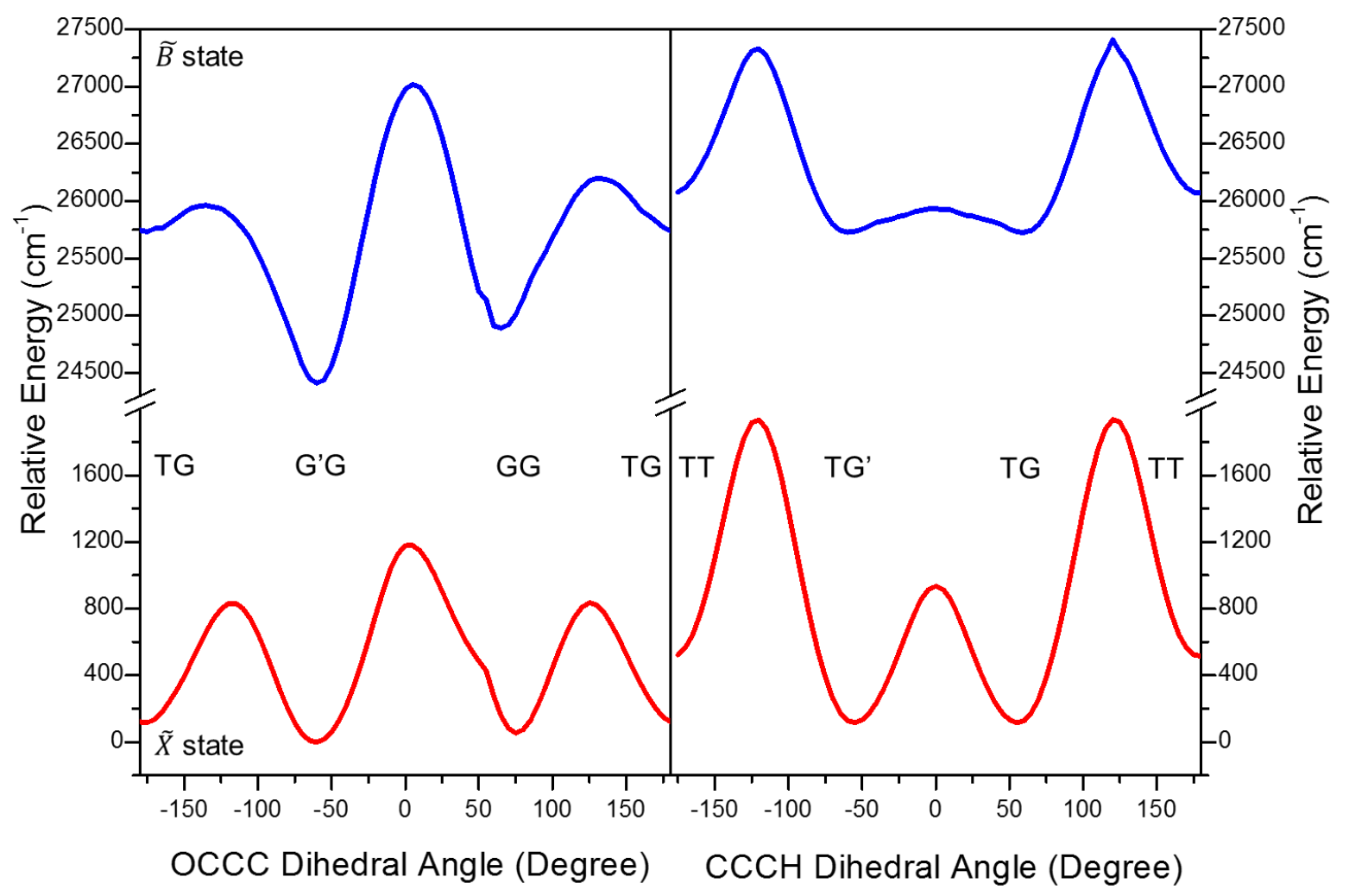

Figure 5.10. Scans of $\tilde{X}$ - and $\tilde{B}$-state potential energy surfaces of the isopentoxy radical along the coordinates of the $\mathrm{OC}_{1} \mathrm{C}_{2} \mathrm{C}_{3}$ and $\mathrm{C}_{1} \mathrm{C}_{2} \mathrm{C}_{3} \mathrm{H}$ dihedral angles. 


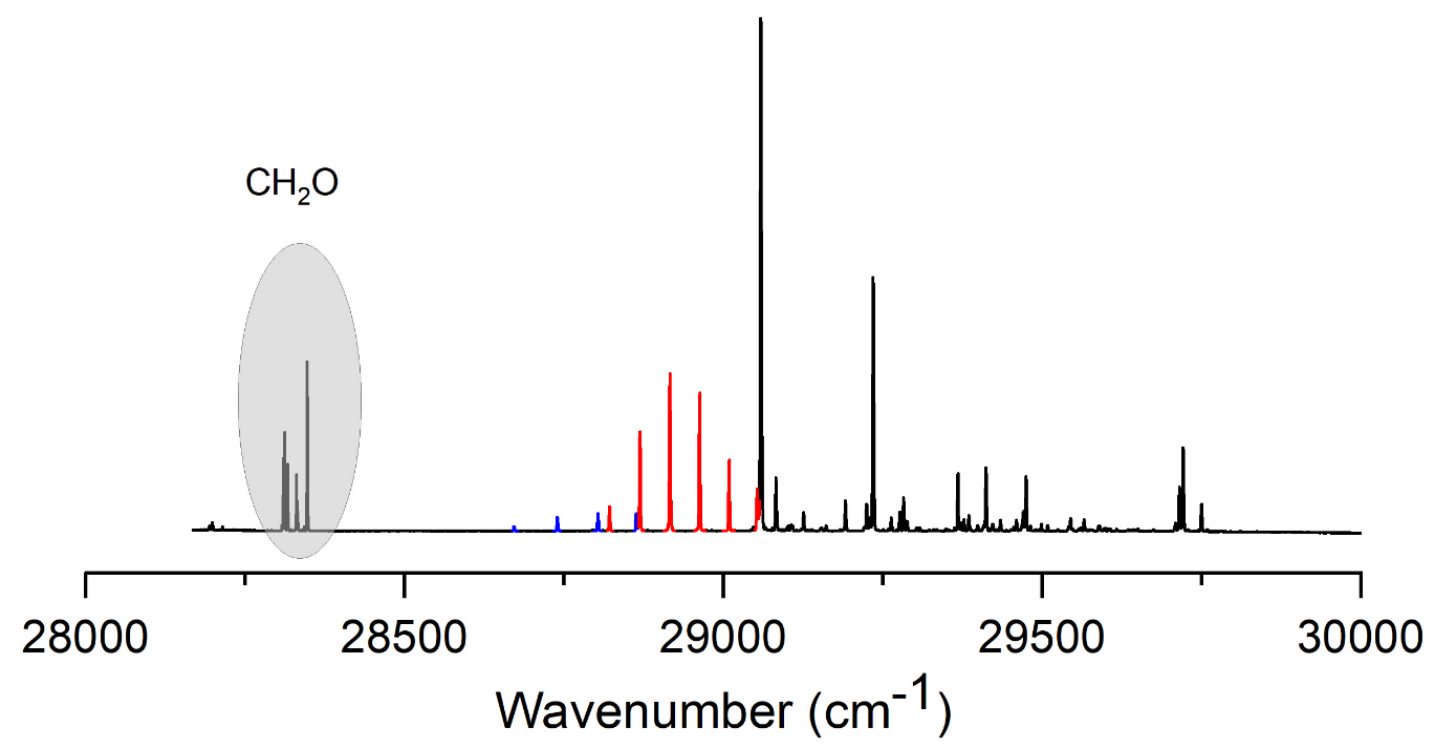

Figure 5.11. LIF spectrum that contains transitions of formaldehyde, a product of $\beta \mathrm{C}$ C fission of isopentoxy. 


\section{CHAPTER VI}

\section{DISPERSED FLUORESCENCE SPECTROSCOPY OF JET-COOLED 2-, 3-, AND 4- METHYLCYCLOHEXOXY RADICALS}

\subsection{Introduction}

The cyclohexoxy radical, like other alkoxy radicals, is an important reaction intermediate in combustion and atmospheric chemistry. $1,2,4,9,94$ The stability of the six-membered ring and its complicated conformational behavior ${ }^{32}$ makes cyclohexoxy particularly interesting in all cyclic alkoxy radicals. From the point of view of theoretical spectroscopy, cyclohexoxy, as well as other cyclic alkoxy radicals, is a prototypical pseudo-Jahn-Teller (pJT) molecule. ${ }^{33,35,137}$ Its two lowest electronic stats $(\tilde{X}$ and $\tilde{A})$ are separated by only 61 $\mathrm{cm}^{-1}$ in energy from each other. ${ }^{33,35}$ Analysis of the rotational and fine structure of the $\tilde{X} /$ $\tilde{A}$ state decomposes the $\tilde{A}-\tilde{X}$ separation into two components: the spin-orbit (SO) splitting and the nonrelativistic separation because of difference in both the electrostatic energy and the zero-point energy (ZPE) between the two states. ${ }^{33,132}$

It is of both theoretical and practical interests to extend the spectroscopic investigation of cyclohexoxy to the methylcyclohexoxy (MCHO) radicals. Especially important is the effect of the methyl group position at the six-membered ring. Previously, 
the $\tilde{B} \leftarrow \tilde{X}$ transitions of all four isomers of MCHO were studied in laser-induced fluorescence (LIF) spectroscopy under jet-cooled conditions. ${ }^{36,37}$ Both the origin band frequencies and vibronic structures of these isomers are different from each other. Such observation confirms the effect of methyl substitution on the $\tilde{B}$ state vibrational structure.

Intramolecular dynamics and chemical kinetics of MCHOs, and indeed alkoxy radicals in general, are also affected by the lowest-energy electronic states $(\tilde{X}$ and $\tilde{A})$. Because all alkoxy radicals can be treated as the alkyl substitution of the methoxy radical $\left(\mathrm{CH}_{3} \mathrm{O}\right)$, these two states are originated from the $\tilde{X}^{2} \mathrm{E}$ state of $\mathrm{CH}_{3} \mathrm{O}$, which is orbitally doubly degenerate. The unpaired electron may occupy either of the two nonbonding orbitals resulting from the $p_{x}$ and $p_{y}$ orbitals of the oxygen atom, which lie within the $\mathrm{OCH}$ plane and perpendicular to it, respectively. In larger alkoxy radicals such as ethoxy, ${ }^{24}$ alltrans conformers of large primary alkoxies, ${ }^{134,135,138}$ isopropoxy ${ }^{26,132}$ and cyclohexoxy, ${ }^{33}$, ${ }^{35}$ the alkyl substitution reduces the molecular symmetry from $C_{3 v}$ (for methoxy) to $C_{s}$ and removes the orbital degeneracy. The $\tilde{X}^{2} \mathrm{E}$ state of the methoxy radical therefore splits into two close-lying states ( $\tilde{X}$ and $\tilde{A}$ ) with $\mathrm{A}^{\prime}$ and $\mathrm{A}^{\prime \prime}$ symmetry, which have the half-filled orbital of the oxygen atom within the $C_{s}$ plane $\left(p_{x}\right)$ and perpendicular to it $\left(p_{y}\right)$, respectively. The alkyl group interacts differently with the $p_{x}$ and $p_{y}$ orbitals, resulting in the $\tilde{A}-\tilde{X}$ separation between the lowest vibrational levels of these two states. The mechanism of interaction between these two close-lying states is of significant theoretical importance. ${ }^{137}$, ${ }^{139,140}$ Especially, the energy ordering of the $\mathrm{A}^{\prime}$ and $\mathrm{A}^{\prime \prime}$ states and the magnitude of the $\tilde{A}-\tilde{X}$ separation depend on the conformation of the free radicals. For gauche conformers of the primary alkoxy radicals ${ }^{88,134,138}$ and secondary alkoxy radicals larger than isopropoxy, ${ }^{141}$ 
the molecular symmetry is further lowered to $C_{1} . \tilde{A}-\tilde{X}$ separation of conformers with $C_{l}$ symmetry of an alkoxy radical is usually smaller than that of $C_{s}$ conformers.

In addition to the lowest vibrational levels, the vibronic structure of the $\tilde{X} / \tilde{A}$ state can be affected by positional isomerization too. In the present work, we report an investigation of the effect of the methyl group position at the six-membered rings of MCHO radicals by dispersed fluorescence (DF) spectroscopy. Experimental DF spectra obtained by pumping different bands in the $\tilde{B} \leftarrow \tilde{X}$ LIF spectra reveal the $\tilde{A}-\tilde{X}$ separation and vibrational structure of the $\tilde{X} / \tilde{A}$ state. By comparing the experimentally observed redshifts with the calculated vibrational frequencies, the vibronic bands in the DF spectra can be assigned to transitions to different vibrational levels of the lower two states. The transition intensities provide further information about the geometry, symmetry, and energetics of the electronic states. The DF spectra of 1-MCHO are significantly different from those of the others because of its tertiary structure and hence deferred to future publication. In the present paper, the results of 2-, 3-, and 4-MCHOs are reported.

\subsection{Quantum Chemical Calculations}

Previously, extensive calculations were performed on the $\tilde{X}$ - and $\tilde{B}$-state 2-, 3-, and 4MCHO radicals and used to guide the analysis of the experimentally obtained LIF spectra. ${ }^{36}$ Such analysis allowed the assignment of vibronic bands in the LIF spectra and suggested that all LIF bands observed under jet-cooled conditions are due to the lowestenergy conformers of each isomer. The six-membered ring in these conformers has chair 
structure, and both the oxygen atom and the methyl group are at the equatorial positions. In the present work, two types of new calculations have been carried out in aid of the analysis of the DF spectra. First, density functional theory (DFT) calculations at the B3LYP/6-31+G(d) level of theory were performed to predict the energy separation between the $\tilde{X}$ and the $\tilde{A}$ states of 4-MCHO, which retains the $C_{s}$ symmetry of the cyclohexoxy radical. This was done by putting the unpaired electron in either $p_{x}$ or $p_{y}$ orbitals localized on the oxygen atom. The calculations predict that the ground electronic state $(\tilde{X})$ has $\mathrm{A}^{\prime \prime}$ symmetry, whereas the first excited state $(\tilde{A})$ is of $\mathrm{A}^{\prime}$ symmetry. The energy separation between these two states was found to be $168 \mathrm{~cm}^{-1}$ (without ZPE corrections). When the molecular symmetry was allowed to be lowered to $C_{l}$, geometry optimization resulted in the pJT-distorted structure of the 4- MCHO isomer. The pJT stabilization energy, defined as the energy difference between the ground-state $\left(\tilde{X}^{2} \mathrm{~A}^{\prime \prime}\right)$ molecule with $C_{s}$ symmetry and that with $C_{1}$ geometry, i.e., no symmetry, was found to be $77 \mathrm{~cm}^{-1}$.

2- and 3-MCHO isomers do not have $C_{s}$ symmetry. The aforementioned technique, therefore, cannot be used to calculate the $\tilde{A}-\tilde{X}$ separation and the pJT stabilization energy. However, an inspection of the molecular orbitals suggests that for the ground electronic state of both isomers, the half-filled orbital is approximately perpendicular to the $\mathrm{OCH}$ plane, viz., the $p_{y}$ orbital. These isomers, therefore, have a ground state with "pseudo-A" symmetry." Figure 6.1 illustrates the half-filled orbitals of 2-, 3-, and 4-MCHOs. All calculations were done using the Gausssian09 software package. ${ }^{128}$

The second type of new calculations on MCHOs are Franck-Condon factor (FCF) calculations for $\tilde{B} \rightarrow \tilde{X}$ and $\tilde{B} \rightarrow \tilde{A}$ transitions. Vibrational modes of the $\tilde{A}$ state (for 4- 
MCHO only) and the $\tilde{X}$ state were calculated at the B3LYP/6-31+G(d) level of theory, whereas those of the $\tilde{B}$ state by configuration interaction singles (CIS) using the same basis set. For the $\tilde{X}$ state of 4-MCHO, the pJT distorted $\left(C_{l}\right)$ geometry was used. Geometries and the displacements of nuclei for the normal modes were calculated using Gaussian09, ${ }^{128}$ and the FCFs and intensities for transitions from the lowest vibrational level of the $\tilde{B}$ state to the vibrational levels of the $\tilde{X} / \tilde{A}$ state were predicted using the ezSpectrum software. ${ }^{121}$ Due to the large difference in $\tilde{X} / \tilde{A}$ - and $\tilde{B}$-state geometries, especially the large increase in $\mathrm{CO}$ bond length upon excitation (e.g., from 1.37 to $1.58 \AA$ for 4- $\mathrm{MCHO}$ ), the Duschinsky rotations were taken into account in the calculation of FCFs. The calculated vibrational frequencies of the $\tilde{X} / \tilde{A}$ state and $\tilde{B} \rightarrow \tilde{X} / \tilde{A}$ FCFs are listed in Tables 6.4-7.

\subsection{Results}

DF spectra of 2-, 3-, and 4-MCHOs were obtained by pumping different vibronic bands of the $\tilde{B} \leftarrow \tilde{X}$ LIF excitation spectra. ${ }^{36}$ The vibronic bands are labeled by capitalized letters following ref. ${ }^{36}$ In this section; we first present the results of 4-MCHO, which has nominal $C_{S}$ symmetry, followed by the 3 - and 2- MCHO radicals.

\subsubsection{4-MCHO}

DF spectra of 4-MCHO (Figure 6.2) were recorded by pumping six vibronic bands of its LIF spectrum, namely, bands A-F. With the origin band (band A at $26906 \mathrm{~cm}^{-1}$ ) pumped, there is a peak in the DF spectrum at $76 \mathrm{~cm}^{-1}$ red-shifted from the excitation wavenumber. 
The intensity of this peak is slightly stronger than the fluorescence emission to the vibrational ground level of the $\tilde{X}$-state, i.e., the zero-red-shift $\tilde{B}^{2} \mathrm{~A}^{\prime} \rightarrow \tilde{X}^{2} \mathrm{~A}^{\prime \prime}$ origin band in the DF spectrum. DFT calculations predict $\tilde{X}$-state vibrational modes with frequencies as low as $109 \mathrm{~cm}^{-1}$. However, the FCF of transition from the vibrational ground level of the $\tilde{B}$-state to this vibrational level is negligible. DFT calculations predict an $\tilde{A}-\tilde{X}$ separation of $168 \mathrm{~cm}^{-1}$ (without ZPE corrections). The DF peak at $76 \mathrm{~cm}^{-1}$ is therefore assigned to the origin band of the $\tilde{B}^{2} \mathrm{~A}^{\prime} \rightarrow \tilde{A}^{2} \mathrm{~A}^{\prime}$ transition.

When bands $\mathrm{C}$ and $\mathrm{E}$ were pumped, low-frequency peaks were observed at 77 and $74 \mathrm{~cm}^{-1}$, respectively. They are assigned to transitions from the corresponding vibrational levels of the $\tilde{B}{ }^{2} \mathrm{~A}^{\prime}$ state to the ground vibrational level of the $\tilde{A}^{2} \mathrm{~A}^{\prime}$ state. The $\tilde{A}-\tilde{X}$ separation of 4-MCHO is therefore determined to be $76(5) \mathrm{cm}^{-1}$, taken as the averaged value of the low-frequency peaks. Intensities of these peaks with bands A, C, and E pumped are close to the zero-red-shift DF origin bands. Noticeably, with bands B, D, and F pumped, the fluorescence emission to the vibrational ground level of the $\tilde{A}$-state is extremely weak (pumping band $\mathrm{B}$ ) or absent (pumping bands $\mathrm{D}$ and $\mathrm{F}$ ). In section 6.2 we will argue that relative intensities of the $\tilde{B} \rightarrow \tilde{X}$ and $\tilde{B} \rightarrow \tilde{A}$ bands are determined by the vibronic symmetry of the pumped LIF bands: The differences in relative intensities of the DF origin bands are due to the fact that bands $\mathrm{A}, \mathrm{C}$, and $\mathrm{E}$ are transitions to a' (symmetric) vibrational levels of the $\tilde{B}$ state, whereas bands $\mathrm{B}, \mathrm{D}$, and $\mathrm{F}$ are to a" (antisymmetric) levels. ${ }^{36}$ Note that transitions to the a" levels are allowed due to the pJT interaction between the $\tilde{A}$ and $\tilde{X}$ states.

Besides the two origin bands, the DF spectrum by pumping band $\mathrm{A}$ is dominated by a progression of strong peaks. The most prominent peaks of each member of the 
progression are found at 1121, 2165, and $3197 \mathrm{~cm}^{-1}$. These three peaks and the $\tilde{B} \rightarrow \tilde{A}$ origin band at $76 \mathrm{~cm}^{-1}$ form a vibrational progression. They are hence assigned to transitions to vibrational levels of the $\tilde{A}$ state. Because of the large difference between the $\tilde{B}$-state and $\tilde{A} / \tilde{X}$-state CO bond lengths, one expects transitions to those lower-state vibrational modes that contain significant $\mathrm{CO}$ stretch character to have large FCFs and hence stronger intensities. Indeed, DFT calculations predict an $\tilde{A}$-state $\mathrm{CO}$ stretch mode with a vibrational frequency of $1060 \mathrm{~cm}^{-1}$, close to the experimentally observed fundamental frequency of $1045 \mathrm{~cm}^{-1}$, taken as the interval between the strongest peak of the second member of the progression and the $\tilde{A} \rightarrow \tilde{X}$ origin band. The strong progression is, therefore, assigned to $\tilde{A}$-state $\mathrm{CO}$ stretch levels (Figure 6.2). FCF calculations predict strong transitions to the $\tilde{A}$-state $\mathrm{CO}$ stretch levels (Figure 6.3). On the contrary, transitions to the $\tilde{X}$-state CO stretch levels are significantly weaker. The reason for weaker transitions to the vibrational levels of the $\tilde{X}$ state is discussed in section 6.4.2.

Second member of the CO stretch progress has three distinctive peaks: $\tilde{v}_{x}=1006$ $\mathrm{cm}^{-1}, \tilde{v}_{y}=1121 \mathrm{~cm}^{-1}$, and $\tilde{v}_{z}=1170 \mathrm{~cm}^{-1}$ (Figure 6.2). Peaks at $\tilde{v}_{x}$ and $\tilde{v}_{z}$ are attributed to transitions to other vibrational levels with $\mathrm{CO}$ stretch character or combination levels of CO stretch mode with low-frequency modes (Figure 6.3). However, definitive assignments are avoided due to the congestion of transitions. In the region of larger redshift $(>1500$ $\mathrm{cm}^{-1}$ ), the third and fourth members of the $\mathrm{CO}$ stretch progression are observable. Six peaks are partially resolved in the region of the first overtone of the $\mathrm{CO}$ stretch (i.e., the third member of the progression, Figure 6.2). They are attributed to the overtone and combination bands of $v_{\mathrm{x}}, v_{\mathrm{y}}$, and $v_{\mathrm{z}}$. The peaks in the region of $3 v_{\mathrm{CO}}(\tilde{A})$ (the fourth member 
of the $\mathrm{CO}$ stretch progression) are unresolved due to the increased density of vibrational levels.

The experimentally observed DF spectrum pumping the LIF origin band is compared with the calculated transition intensities and simulated spectra in Figure 6.3. In the simulation, the $\tilde{B} \rightarrow \tilde{A}$ origin band is shifted to the red with respect to the $\tilde{B} \rightarrow \tilde{X}$ origin band by the experimentally determined $\tilde{A}-\tilde{X}$ separation. The intensities of the $\tilde{B} \rightarrow \tilde{X}$ and $\tilde{B} \rightarrow \tilde{A}$ transitions are scaled so that the relative intensities of the origin bands match the experimentally obtained spectrum. The simulation semiquantitatively reproduces the spectrum in terms of both transition frequencies and intensities. The calculations correctly predict that the $\tilde{B} \rightarrow \tilde{A}$ transitions to vibrationally excited levels are significantly stronger than the $\tilde{B} \rightarrow \tilde{A}$ transitions. However, although the FCF calculation predicts several weak transitions other than origin bands and the CO-stretch progression, these transitions were not observed in the DF spectrum.

When LIF bands other than A were pumped, the emission to the $\mathrm{CO}$ stretch levels (bands $\mathrm{x}, \mathrm{y}, \mathrm{z}$, as well as their overtone and combination bands) is much weaker except in the case of pumping band E. Band E was assigned to the transition to the $v_{27}$ level of the $\tilde{B}$ state ${ }^{36}$ which has significant $\mathrm{CO}$ stretch character. The presence of the transitions to the CO stretch levels of the $\tilde{A}$-state with band E pumped can, therefore, be again explained by large FCFs. On the contrary, significantly smaller FCFs between other pumped vibrational modes (for bands B, C, D, F) and the CO stretch mode are expected. 
With bands B-F pumped, DF bands other than the origin bands appear in the lowfrequency region $\left(<1000 \mathrm{~cm}^{-1}\right)$ as labeled by lowercase letters $\mathrm{b}-\mathrm{f}$ in Figure 6.2. The redshifts of these peaks relative to the $\tilde{B} \rightarrow \tilde{A}$ origin band are close to (but not exactly equal to) the LIF pumping frequencies relative to the $\tilde{B} \leftarrow \tilde{X}$ origin band (Table 6.1). This is due to the large vibrational wave function overlap between the $\tilde{A}$ and $\tilde{B}$-state levels of the same, non-CO-stretch mode because the only significant geometry change upon excitation is the elongation of the $\mathrm{CO}$ bond and mixing between non-CO-stretch vibrational modes is expected to be small. The difference between the redshift of the DF band (with respect to the $\tilde{B} \rightarrow \tilde{A}$ origin) and the relative frequency of the pumped LIF band (relative to the $\tilde{B} \leftarrow$ $\tilde{X}$ origin, i.e., band A) is therefore attributed to the small difference between the $\tilde{A}$ - and $\tilde{X}$ state vibrational frequencies of the same mode. DF transitions to the $\tilde{X}$-state levels of these modes were also observed, although the intensities are much lower. The disparity between the $\tilde{B} \rightarrow \tilde{X}$ and $\tilde{B} \rightarrow \tilde{A}$ transition intensities will be discussed in section 6.4.2. $\tilde{A}-\tilde{X}$ separation of these vibrational levels are close to that of the vibrational ground level, except for that of band e, which is $100 \mathrm{~cm}^{-1}$ (Table 6.1).

In the DF spectrum with band $\mathrm{F}$ pumped, the peak at $339 \mathrm{~cm}^{-1}$ coincides in redshift with band $b$ in the DF spectrum pumping band B. It is hence labeled b' in Figure 6.2. Such an observation confirms the previous assignment of band $\mathrm{F}$ to a combination band of the vibrational mode of band $\mathrm{B}\left(v_{55}\right)$ and another vibrational mode $\left(v_{30}\right) \cdot{ }^{36} \mathrm{Band} \mathrm{b}^{\prime}$ is therefore assigned to the transition to $v_{55}$ vibrational level of the $\tilde{A}$ state, whereas the other DF peak in the low-frequency region (band f) to the $v_{55}+v_{30}$ level of the $\tilde{A}$ state. 
The most noticeable difference between DF spectra obtained by pumping the LIF origin band (band A) and other vibronic bands is that the prominent vibrational progression observed in the former case is shifted to the red in the latter case. The amount of such a redshift is equal to the redshift of the FC favored transition (bands $b-f$ ) with respect to the $\tilde{B} \rightarrow \tilde{A}$ origin band, i.e., the $\tilde{A}$-state vibrational frequency of the pumped mode. For instance, in the DF spectrum with band E pumped, the frequency interval between the $\tilde{B}$ $\rightarrow \tilde{A}$ origin band $\left(74 \mathrm{~cm}^{-1}\right)$ and band $\mathrm{e}\left(648 \mathrm{~cm}^{-1}\right)$ matches that between the $\tilde{A}$ state $\mathrm{CO}$ stretch band $\left(1124 \mathrm{~cm}^{-1}\right)$ and the strong peak at $1705 \mathrm{~cm}^{-1}$ within the error bars (Table 6.1). Therefore, if the DF spectra are shifted to the blue by the observed $\tilde{A}$-state frequencies of the pumped modes so that bands $\mathrm{b}-\mathrm{f}$ are aligned with the $\tilde{B} \rightarrow \tilde{A}$ origin band, all DF spectra of 4-MCHO will have the same vibrational progressions (Figure 6.7). Such an observation implies that when a vibronic level other than the $\mathrm{CO}$ stretch levels is pumped, the transition with the largest FCF is not to the $\tilde{A}$-state CO stretch level or the vibrational level of the pumped mode, but to the combination level of the $\mathrm{CO}$ stretch mode and the pumped mode. Figure 6.7 also confirms that all strong peaks observed in the DF spectra except the zerored-shift bands should be assigned to transitions to the $\tilde{A}$-state levels rather than the $\tilde{X}$-state levels.

Redshifts of DF bands of 4-MCHO are summarized in Table 6.1 with their assignments. To elucidate the vibrational assignments, the relative wavenumbers with respect to the $\tilde{B} \rightarrow \tilde{A}$ origin or $\tilde{A}$-state vibrational levels (bands $\mathrm{b}-\mathrm{f}$ ) are also listed. 


\subsubsection{3-MCHO}

DF spectra of the 3-MCHO isomer (Figure 6.4) were recorded by pumping six LIF bands: the origin band $\mathrm{A}$, fundamental bands $\mathrm{B}$ and $\mathrm{C}, \mathrm{CO}$-stretch band $\mathrm{A}^{*}$, and bands $\mathrm{B}^{*}$ and $\mathrm{C}^{*}$, which are combination bands of vibrational modes of bands B and C with the $\mathrm{CO}$ stretch mode. The averaged $\tilde{A}-\tilde{X}$ separation by pumping the LIF bands is $62(5) \mathrm{cm}^{-1}$. The relative intensities of the $\tilde{B} \rightarrow \tilde{A}$ and $\tilde{B} \rightarrow \tilde{X}$ origin bands are different with different LIF bands pumped. With band A(the LIF origin band) pumped, the origin band of the $\tilde{B} \rightarrow \tilde{A}$ transition in the DF spectrum is stronger than that of the $\tilde{B} \rightarrow \tilde{X}$ transition. Similar relative intensities were observed in the DF spectrum of 4-MCHO when the LIF origin band was pumped (Figure 6.2), suggesting similar electron configurations for the $\tilde{A} / \tilde{X}$ state of these two isomers. This has been predicted by DFT calculations (Figure 6.1). With bands B and C pumped, the relative intensities of the two origin bands in the DF spectra are reversed: the $\tilde{B} \rightarrow \tilde{A}$ origin band is significantly weaker than the $\tilde{B} \rightarrow \tilde{X}$ one. This is similar to the DF spectra of 4-MCHO with a" levels of the $\tilde{B}$ state pumped, in which the $\tilde{B} \rightarrow \tilde{A}$ transitions are extremely weak or absent. Such reversion, therefore, suggests that vibrational modes of bands $\mathrm{B}$ and $\mathrm{C}$ of 3-MCHO would be antisymmetric $\left(\mathrm{a}^{\prime \prime}\right)$ if the methyl group was replaced by a hydrogen atom.

When band $\mathrm{A}$ is pumped, a strong progression is observable and assigned to transitions to $\mathrm{CO}$ stretch levels. The most prominent peaks in the members of the progression are at 1105,2113 , and $3120 \mathrm{~cm}^{-1}$. They are transitions to the $\tilde{A}$ state $\mathrm{CO}$ stretch levels based on the energy intervals. The peak at $1028 \mathrm{~cm}^{-1}$ is assigned tentatively to the $\tilde{X}$-state CO stretch level. The $\tilde{A}-\tilde{X}$ separation of the CO stretch level so determined is larger 
than that of the vibrational ground levels $\left(77 \mathrm{~cm}^{-1}\right.$ vs. $\left.62 \mathrm{~cm}^{-1}\right)$, a phenomenon that was also observed in the DF spectrum of tert-butoxy. ${ }^{142}$ Simulation using Gaussian09 and ezSpectrum (Figure 6.3) correctly predicts strong transitions in the $\mathrm{CO}$ stretch region. However, the predicted frequency of the $\mathrm{CO}$ stretch mode is not as accurate as for the 4MCHO isomer. In addition, they predict overtone transitions weaker than the fundamental, whereas, in the experimentally obtained spectrum, the overtone bands are stronger.

A doublet (b or c) was observed in the DF spectra when either band B or C was pumped, corresponding to the transition to the vibrational levels of the pumped mode in the $\tilde{A}$ and $\tilde{X}$ states (bands $\mathrm{b}$ and $\mathrm{c}$ in Figure 6.4). Unlike in the case of 4-MCHO, for which transitions to the $\tilde{X}^{2} \mathrm{~A}^{\prime \prime}$ state vibrational levels are completely absent or much weaker than to the $\tilde{A}^{2} \mathrm{~A}^{\prime}$ state, the two peaks of the doublets in the 3-MCHO DF spectra are of comparable intensities, although the $\tilde{B} \rightarrow \tilde{A}$ transitions are still stronger than the $\tilde{B} \rightarrow \tilde{X}$ ones. This is due to the lowered symmetry and hence, less strict selection rules (section 6.2). Similar to 4-MCHO, with bands B and $\mathrm{C}$ pumped, the DF spectra of 3-MCHO are dominated by progressions of combination bands of $\mathrm{CO}$ stretch and the pumped modes.

When band $\mathrm{A}^{*}$ (CO-stretch band) was pumped, a strong $\mathrm{CO}$ stretch progression was observed. The DF spectrum by pumping band B* (combination band) is dominated by a progression of combination bands of $\mathrm{CO}$ stretch and the pumped mode. In both cases, the fourth member of the progressions was obscured by the unstructured DF signal, most probably due to excited-state intramolecular vibrational energy redistribution (IVR). ${ }^{34}$ The first member of the progression in the DF spectrum pumping band $\mathrm{B}^{*}$ is labeled $\mathrm{b}^{\prime}$ in Figure 
6.4. Such progression becomes very weak when band $C^{*}$ is pumped. Its first member is labeled $\mathrm{c}^{\prime}$ in Figure 6.4.

Furthermore, compared to the DF spectra by pumping band A, B, and C, new peaks are observable in those pumping bands $\mathrm{A}^{*}, \mathrm{~B}^{*}$, and $\mathrm{C}^{*}$. They are asterisked in Figure 6.4. In each spectrum, these new peaks form a second progression that is red-shifted by $\sim 680$ $\mathrm{cm}^{-1}$ from the aforementioned progression of the $\mathrm{CO}$ stretch bands or combination bands of the $\mathrm{CO}$ stretch. Such a redshift matches the $\mathrm{CO}$ stretch frequency of the $\tilde{\mathrm{B}}$ state, determined to be $683 \mathrm{~cm}^{-1}$ in the LIF spectrum. ${ }^{36}$ It can, therefore, be explained by excitedstate vibrational population relaxation: After LIF band $\mathrm{A}^{*}$ (the $\mathrm{CO}$ stretch band) is pumped, part of its population relaxes to the vibrational ground state of the $\tilde{B}$ state. Fluorescence from both the $\mathrm{CO}$ stretch level and the vibrational ground level are therefore possible. Because fluorescence from both levels was observed with comparable intensities, the vibrational relaxation process and fluorescence process must have comparable time scales, i.e., at the microsecond scale. The mechanism is illustrated in Figure 6.5. When combination bands $\mathrm{B}^{*}\left(v_{\mathrm{CO}}+v_{54}\right)$ and $\mathrm{C}^{*}\left(v_{\mathrm{CO}}+v_{51}\right)$ are pumped, the dominant relaxation process is not to the vibrational ground level, but the non-CO-stretch vibrational level ( $v_{54}$ or $v_{51}$ ) (Figure 6.5). In the former case (pumping band $\mathrm{B}^{*}$ ), the relaxation and fluorescence processes are of comparable rates as fluorescence from both the initial vibrational level $\left(v_{\mathrm{CO}}+v_{54}\right)$ upon LIF excitation and from the level after relaxation $\left(v_{54}\right)$ are observed. In the latter case (pumping band $\left.\mathrm{C}^{*}\right)$, the DF signal from the initial vibrational level $\left(v_{\mathrm{CO}}+v_{51}\right)$ is very weak, suggesting a rapid relaxation process that outpaces the fluorescence process. 
Redshifts and relative wavenumbers of DF bands of 3- $\mathrm{MCHO}$ are summarized in Table 6.2 with their assignments. Figure 6.8 illustrates the DF spectra of 3-MCHO when they are blue-shifted by the $\tilde{A}$ state frequency of the pumped non-CO-stretch modes. The progressions are aligned in the shifted spectra.

\subsubsection{2-MCHO}

DF spectra of the 2-MCHO isomer (Figure 6.6) were recorded by pumping six LIF bands, labeled A-F in ref. ${ }^{36}$ The $\tilde{A}-\tilde{X}$ separation of $2-\mathrm{MCHO}$ is $110(5) \mathrm{cm}^{-1}$, determined as the energy separation between the first two strong peaks in the DF spectra. Similar to 3- and 4-MCHOs, the relative intensities of these two peaks vary depending on the LIF peaks pumped. Transitions to $\tilde{A}$ - and $\tilde{X}$-state levels of the pumped vibrational modes were also observed (bands $\mathrm{b}-\mathrm{f}$ in Figure 6.6). Also observed are the $\tilde{A}$-state $\mathrm{CO}$ stretch progression (when band A was pumped) or progressions of combination bands of $\mathrm{CO}$ stretch and the pumped modes (when bands B-F were pumped). FCFs calculations qualitatively reproduce the experimental spectrum (Figure 6.3). Figure 6.9 illustrates the coincidence of progressions in the DF spectra after the blue shift. Table 6.3 summarizes redshifts, relative wavenumbers, and assignments.

In the case of pumping band $\mathrm{F}$, a peak at $232 \mathrm{~cm}^{-1}$ red shift was observed in the DF spectrum. DFT calculation does predict an $\tilde{X}$ state methyl torsion mode of $229 \mathrm{~cm}^{-1}$ for 2MCHO. Band F was previously assigned to $v_{47}$, the ring breathing mode. It is unlikely that these two modes are connected by considerable off-diagonal FCF. Band F in the LIF 
spectrum should, therefore, probably be reassigned to the combination band of methyl torsion (calculated to be $230 \mathrm{~cm}^{-1}$ ) and $v_{54}$ (calculated to be $288 \mathrm{~cm}^{-1}$ and illustrated in Figure 6.10).

\subsection{Discussion}

\subsubsection{Conformational and Vibrational Assignments}

In ref. ${ }^{36}$, LIF bands of 2-, 3-, and 4-MCHOs were assigned to the lowest-energy conformer of each isomer with diequatorial structure. In the present work, the single-conformer assignments have been confirmed by the experimentally obtained DF spectra on the basis of the similarity between DF spectra pumping different LIF bands of each isomer.

In the cases of all three isomers, DF spectra are dominated by a $\mathrm{CO}$ stretch progression when the origin band or the CO stretch band in the LIF spectrum (e.g., bands A and $\mathrm{A}^{*}$ of 3- $\mathrm{MCHO}$ ) is pumped. This has been observed in DF spectra of all primary and secondary chain alkoxy radicals,,${ }^{34}$ as well as cyclohexoxy, ${ }^{35}$ which was attributed to the large FCFs for such transitions because of the large difference in $\tilde{X} / \tilde{A}$-and $\tilde{B}$-state CO bond lengths ( $\left.\Delta R_{\mathrm{CO}} \sim 0.2 \AA\right)$. When other vibronic bands in the LIF spectra were pumped, DF transitions to the ground state vibrational level of the same vibrational mode as the pumped band were observed because of large FCFs of such transitions. Also observed were combination bands of the pumped mode with the $\mathrm{CO}$ stretch modes. The combination bands have even higher intensities than the fundamental band of the pumped mode. Such a phenomenon was previously observed in the DF spectra of cyclohexoxy ${ }^{34}$ and can also 
be explained on the basis of the evaluation of FCFs: The large difference between CO bond lengths of the $\tilde{X} / \tilde{A}$ - and the $\tilde{B}$-states causes rotation of vibrational normal coordinates upon electronic excitation. The ground-state normal coordinates of vibrational modes (other than $\mathrm{CO}$ stretch) are mixed with those of the CO stretch modes in the excited state (Duschinsky mixing), and they are coupled in the vibrational overlap integrals. As a result, when nonCO-stretch vibrational levels of the $\tilde{B}$ state are pumped, the DF transitions with the largest FCFs are those to the combination levels of the pumped mode and CO stretch. Similar combination bands were observed in other molecules with large geometry change upon excitation, for instance, in the photoelectron spectra of the $\tilde{B}^{2} \mathrm{~B}_{2}$ state of $\mathrm{H}_{2} \mathrm{O}^{+}$: Because of the large change in the $\angle \mathrm{HOH}$ angle between the neutral molecule and the cation $\left(104^{\circ} \mathrm{vs}\right.$ $57^{\circ}$ ), pure excitation of the $v_{1}$ vibrational mode (symmetric stretch) is not observed. Instead, the photoelectron spectra are dominated by combination bands of $v_{1}$ and $v_{2}$ (bending mode). ${ }^{143},{ }^{144}$ Another example that, like MCHOs, also involves vibronic interactions is the Herzberg-Teller (HT)-allowed $\tilde{A}^{1} \mathrm{~B}_{2 u} \leftarrow \tilde{X}^{1} \mathrm{~A}_{1 \mathrm{~g}}$ transition of benzene, in which the HT mode $v_{6}\left({ }^{\mathrm{e}}{ }_{\mathrm{g}} \mathrm{g}\right)$ reveals itself in combination with the totally symmetric $v_{1}\left({ }^{\mathrm{a}} 1_{\mathrm{g}}\right)$ because of the large difference in $\mathrm{C}-\mathrm{C}$ bond length between the $\tilde{X}$ and the $\tilde{A}$ state $\left(\Delta r_{\mathrm{CC}}=\right.$ $0.0353 \AA) \cdot{ }^{145}$

It is worth noting that the Duschinsky mixing is closely related to vibronic interactions. It has been argued theoretically that vibronic interaction, such as the HT effect, introduces the Duschinsky rotation and breaks the mirror symmetry between the fluorescence and the absorption spectra because of the effect of Duschinsky mixing on transition intensities should be more pronounced for transitions to vibronically coupled 
states than those from them. ${ }^{146}$ Because of the similarity between the HT effect and the pJT effect, such a difference is expected for pJT molecules such as MCHOs too. Moreover, theory also shows that spectroscopic manifestations of the Duschinsky mixing are likely to be more pronounced in molecules with lower symmetry and larger size, ${ }^{147}$ which may explain the observation of the "shift effect" (Figures 6.7-9) in the DF spectra of MCHOs and cyclohexoxy but not the methoxy radical and straight-chain alkoxy radicals. Although a theoretical analysis of the connection between the pJT effect and the Duschinsky mixing is out of the scope of the present work, the experimentally observed DF spectra suggest a close relation between these two effects.

\subsubsection{Symmetry of $\tilde{A} / \tilde{X}$ States and Transition Intensities}

Previously, the symmetries of the $\tilde{A}$ / $\tilde{X}$ state of primary and secondary chain alkoxies with Cs geometry as well as cyclohexoxy were determined experimentally on the basis of the analysis of the rotational structure. It has been concluded that the $\tilde{X}$ state of ethoxy, ${ }^{24}$ alltrans conformers of 1-propoxy, as well as other larger primary alkoxies, ${ }^{88,134,138}$ and cyclohexoxy ${ }^{33}$ are of $\mathrm{A}^{\prime \prime}$ symmetry, whereas that of isopropoxy is an $\mathrm{A}^{\prime}$ state. ${ }^{132}$ The differences were explained by the interaction between the $p_{x}$ (in the $C_{s}$ plane) and $p_{y}$ (perpendicular to the $C_{s}$ plane) orbitals of the oxygen atom and the alkyl group, which stabilizes the free radical. The overlap of a fully occupied p-orbital with the methyl group has a more significant stabilization effect than a half-filled one. In the case of primary alkoxy radicals and the cyclohexoxy radicals, the $p_{x}$ orbital is extended toward the alkyl 
groups. The electron configuration with fully occupied $p_{x}$ orbital and half-filled $p_{y}$ orbital, therefore, has lower energy so that the ground electronic state has $\mathrm{A}^{\prime \prime}$ symmetry. For isopropoxy, the $p_{y}$ orbital has a better overlap with the two methyl groups, and hence the electronic state with $\mathrm{A}^{\prime}$ symmetry has lower energy. Interaction between the $\mathrm{O}$ atom and the methyl groups in 4-MCHO is similar to that of cyclohexoxy; a $\tilde{X}^{2} \mathrm{~A}^{\prime \prime}$ state is therefore expected, which is consistent with quantum chemical calculations.

No rotationally resolved spectra of 4-MCHO have been obtained. Therefore, definitive experimental evidence of its $\tilde{X} / \tilde{A}$ state symmetry is still elusive. However, relative intensities of the $\tilde{B} \rightarrow \tilde{X}$ and $\tilde{B} \rightarrow \tilde{A}$ origin bands in the DF spectra (Figure 6.2) support the conjecture above that the ground electronic state of 4-MCHO has A" symmetry. Theoretical analysis of the electric transition dipole moment (TDM) for the $\tilde{B}-\tilde{X}$ and $\tilde{B}-\tilde{A}$ transitions was provided in refs. ${ }^{33,}{ }^{132}$ The $\tilde{B}^{2} \mathrm{~A}^{\prime}-\tilde{X} / \tilde{A}^{2} \mathrm{~A}^{\prime} \mathrm{TDM}$ is dominated by the HT type effect because of the difference between the permanent dipole moment (PDM) of the upper and lower states. Because the difference in PDM is mainly due to the large variation of the $\mathrm{CO}$ bond length upon excitation, the HT-induced TDM is oriented approximately along with the $\mathrm{CO}$ bond, defined to be the z-axis. Based on symmetry argument, the TDM of the $\tilde{B}^{2} \mathrm{~A}^{\prime}-\tilde{X} / \tilde{A}^{2} \mathrm{~A}^{\prime \prime}$ transition is along the y-axis, defined as perpendicular to the Cs plane, and has the same magnitude as the $\mathrm{x}$-component of the TDM of the $\tilde{B}^{2} \mathrm{~A}^{\prime}-\tilde{X} / \tilde{A}^{2} \mathrm{~A}^{\prime}$ transition, which is much smaller than its z component. The $\tilde{B}{ }^{2} \mathrm{~A}^{\prime}-\tilde{X} / \tilde{A} \mathrm{~A}^{\prime \prime} \mathrm{TDM}$ is therefore expected to be significantly smaller than that of the $\tilde{B}^{2} \mathrm{~A}^{\prime}-\tilde{X} / \tilde{A} \mathrm{~A}^{\prime}$ transition if no coupling between the $\tilde{A}$ and $\tilde{X}$ states is involved. Although coupling between these two states via SO interaction reduces the difference between the intensities of the $\tilde{B}^{2} \mathrm{~A}^{\prime}-\tilde{X} / \tilde{A}$ 
${ }^{2} \mathrm{~A}^{\prime}$ and $\tilde{B}^{2} \mathrm{~A}^{\prime}-\tilde{X} / \tilde{A}^{2} \mathrm{~A}^{\prime \prime}$ transitions, ${ }^{122}$ the former is still of stronger intensity than the latter. In addition, the zero-red-shift peaks may contain scattering of the LIF excitation laser, although the gate delay time in the DF experiment was significantly long compared to the laser pulse duration. The relatively stronger $\tilde{B}-\tilde{A}$ origin band in the DF spectrum of 4MCHO with the LIF origin band pumped, therefore, supports the prediction of quantum chemical calculations that its ground electronic state has A" symmetry.

When symmetric vibrational modes of the $\tilde{B}$ state of 4 - MCHO are pumped, using a similar symmetry argument, the $\tilde{B} \rightarrow \tilde{A}$ origin band in the DF spectra is expected to be stronger than the $\tilde{B} \rightarrow \tilde{X}$ one the same as when the LIF origin band is pumped. On the contrary, when antisymmetric vibrational modes are pumped, the relative intensities are reversed: the $\tilde{B}-\tilde{X}$ band has a higher intensity. The intensity pattern can, therefore, be used to determine the vibrational symmetry of the upper level of the LIF transition. Such a conclusion is consistent with all vibronic assignment in ref. ${ }^{36}$ except for band D of 4MCHO at $441 \mathrm{~cm}^{-1}$ relative to the LIF origin band), which was assigned to $v_{28}\left(\mathrm{a}^{\prime}\right)$. We reassign band $\mathrm{D}$ to $v_{53}\left(\mathrm{a}^{\prime \prime}\right)$, predicted to be at $463 \mathrm{~cm}^{-1}$ by CASSCF calculations. ${ }^{36}$

The 2- and 3-MCHO radicals do not have $C_{s}$ symmetry. However, the calculations also predict that, like 4-MCHO, their $\tilde{X}$ states have the half-filled $p_{x}$ orbital oriented perpendicular to the $C_{s}$ plane, i.e., a "pseudo-A" state" with half-filled $p_{y}$ orbitals. Such a prediction has been confirmed by the DF spectra of these two isomers with the LIF origin band pumped, in which the $\tilde{B}-\tilde{A}$ origin bands have higher intensity. 
In the DF spectra of all three isomers, fluorescence emissions to vibrationally excited levels are dominated by transitions to the $\tilde{A}$ rather than the $\tilde{X}$ state levels. In the case of 4-MCHO, the latter transitions are often unobservable, probably because of the high symmetry of 4-MCHO compared to the other two isomers. As discussed above, the $\mathrm{A}^{\prime \prime}$ state steals intensity from the $\mathrm{A}^{\prime}$ state through $\mathrm{SO}$ interaction. The observed difference in transition intensities between DF transitions to the vibrational ground levels and vibrationally excited levels of the $\tilde{A} / \tilde{X}$ state may suggest smaller SO interaction between the vibrationally excited levels of the $\tilde{A}$ and $\tilde{X}$ states than their vibrational ground levels.

\subsection{3 $\tilde{A}-\tilde{X}$ Separation}

The experimentally determined energy separation between the $\tilde{A}$ and $\tilde{X}$ states and their symmetries can be used to test ab initio calculations. In the previous works on ethoxy, ${ }^{24}$ isoproxpoxy ${ }^{132}$ and cyclohexoxy, ${ }^{33}$ DFT calculations correctly predicted the energy ordering of the $\mathrm{A}^{\prime}$ and $\mathrm{A}^{\prime \prime}$ states and semiquantitatively the magnitude of the $\tilde{A}-\tilde{X}$ separation. In the present work on 4-MCHO the DFT calculations demonstrate the same level of accuracy. For isomers without $C_{s}$ symmetry, 2-MCHO and 3-MCHO, such strategy does not work. Prediction of $\tilde{A}-\tilde{X}$ separations using other quantum chemical methods such as CASSCF are in process.

Within the three isomers studied in the present work, 2- MCHO shows a uniquely large $\tilde{A}-\tilde{X}$ separation as well as smaller $\tilde{B}-\tilde{X}$ excitation energy. ${ }^{36}$ Both observations may be explained by the interaction between the oxygen atom and the methyl group: Of the three 
isomers, the methyl group is closest to the oxygen atom in 2-MCHO and hence the strongest interaction.

\subsection{Conclusions}

DF spectra of 2-, 3-, and 4-MCHO radicals have been observed under jet-expansion conditions. Some important information about MCHO radicals has been obtained in analyzing the DF spectra, including (i) the energy separations between ground vibrational levels of the ground electronic state $(\tilde{X})$ and the low-lying electronic state ( $\tilde{A}$ ), (ii) the vibrational structure of these two states, and (iii) relative intensities of transitions from the vibronic levels of the high-lying $\tilde{B}$ state to the vibrational levels of the lower states. The DF spectra are dominated by the $\mathrm{CO}$ stretch progression when the origin band or the $\mathrm{CO}$ stretch band of the LIF spectra is pumped. When other vibronic bands are pumped, progressions of the $\mathrm{CO}$ stretch modes combined with the pumped mode are observed. The experimentally obtained spectra provide benchmarks to quantum chemical calculations in terms of energies of electronic states, vibrational frequencies in pJT-coupled states, and FCFs of vibronic transitions to such states. Evidence for vibrational population relaxation in the $\tilde{B}$ state following photoexcitation has been observed. Such relaxation is from the $\mathrm{CO}$ stretch level to the ground vibrational level or from combination levels of $\mathrm{CO}$ stretch and other vibrational modes to those of the non-CO stretch modes. 


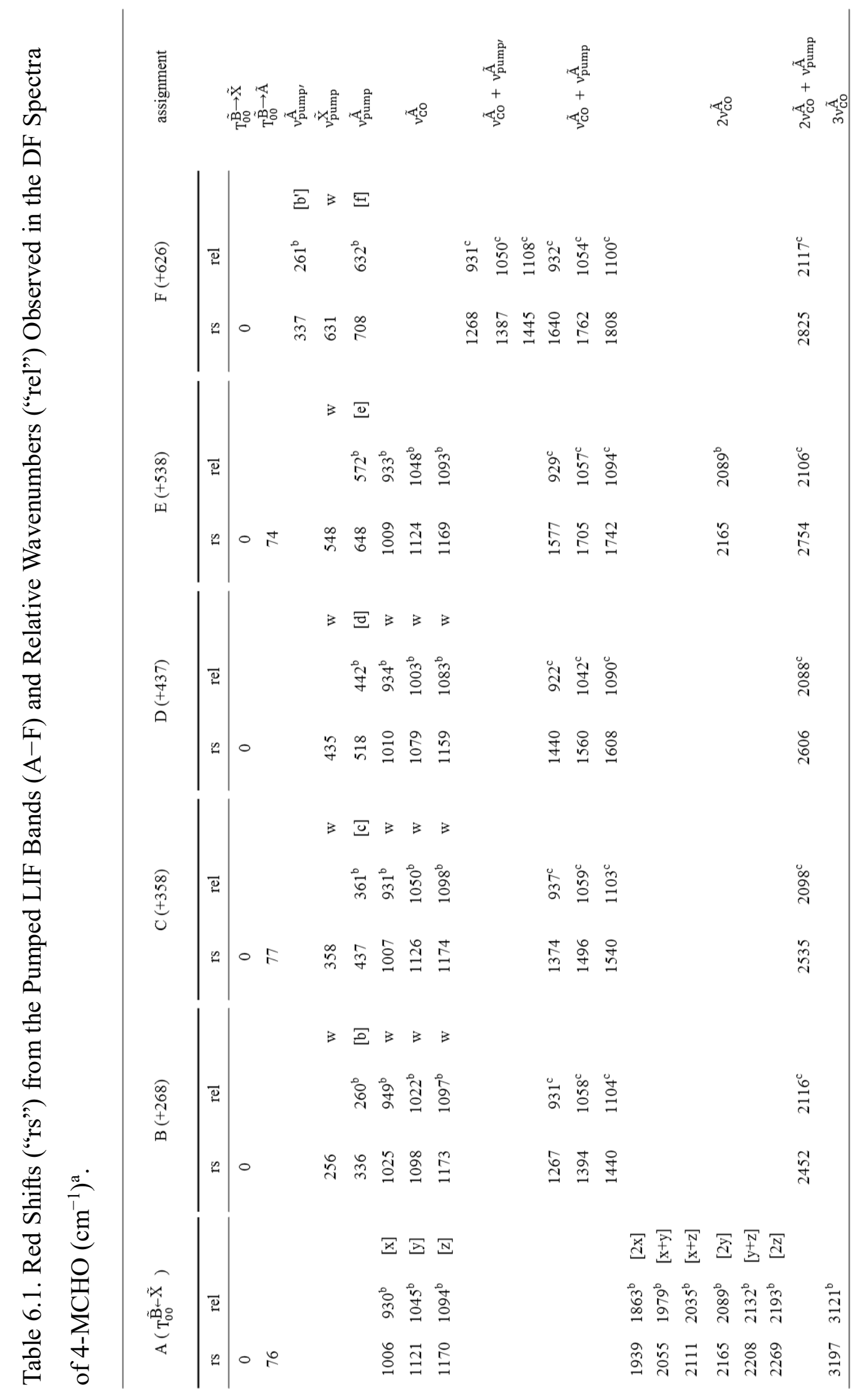


${ }^{a}$ Relative wavenumbers are calculated to elucidate vibrational assignments (see footnotes).

Letters in squared brackets correspond to labels in Figure 6.2.

Letter " $w$ " denotes weak DF bands.

${ }^{\mathrm{b}}$ Redshift relative to the averaged value of $\tilde{A}-\tilde{X}$ separation $\left(76 \mathrm{~cm}^{-1}\right)$. The relative wavenumber corresponds to the $\tilde{A}$-state vibrational level.

c Redshift relative to the $\tilde{A}$-state vibrational frequency of the pumped mode $v_{\text {pump. }}$ The relative wavenumber corresponds to the lower-state $\mathrm{CO}$ stretch level. When the combination band $\mathrm{F}$ was pumped, two progressions were observed, corresponding to $v_{\text {pump }}$ and $v_{\text {pump }}$. The relative wavenumbers for the former and later progressions are calculated with respect to band $f$ and band $b^{\prime}$, respectively. 


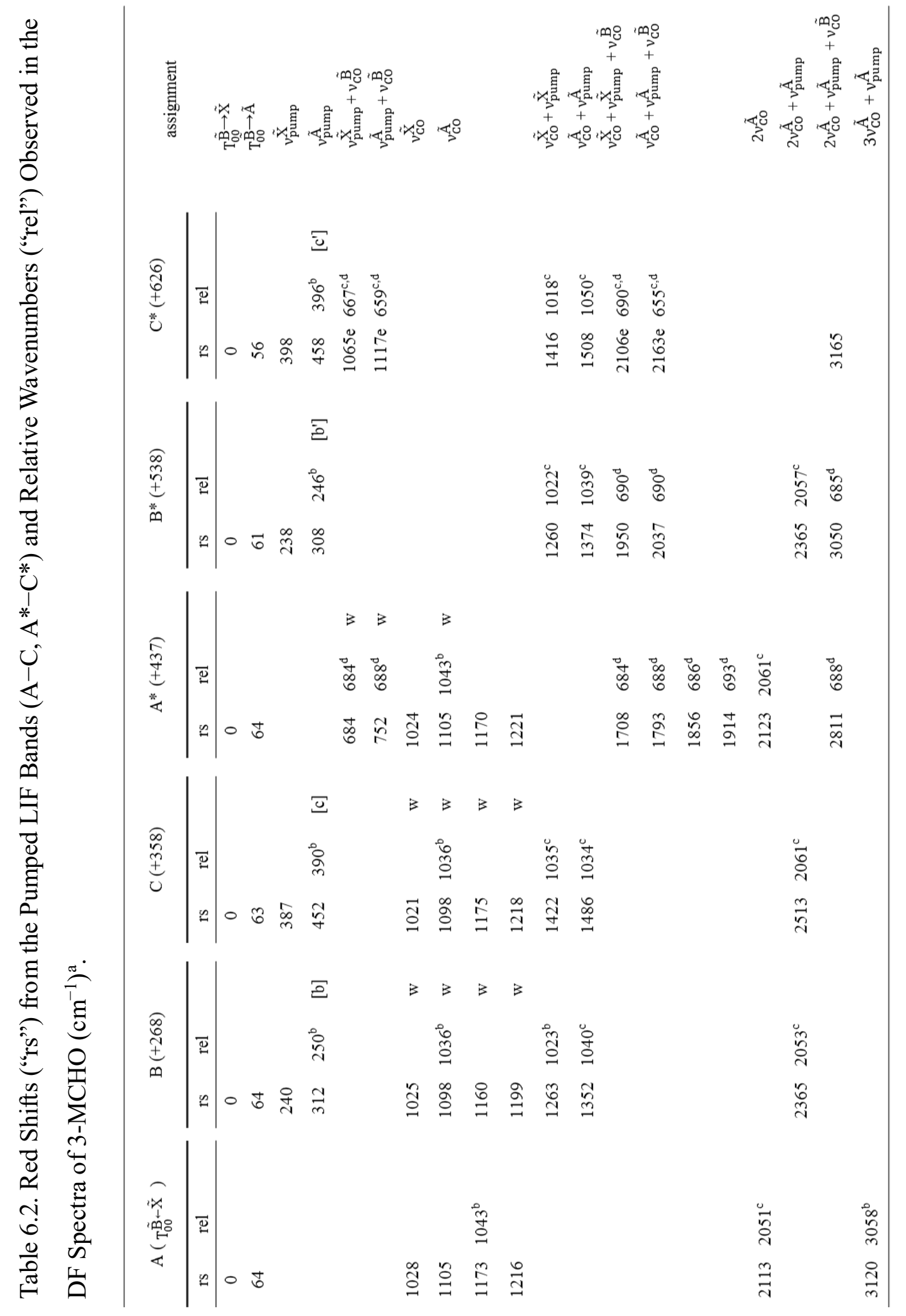


${ }^{\text {a }}$ Letters in square brackets correspond to labels in Figure 6.4.

${ }^{\mathrm{b}}$ Redshift relative to the averaged value of the $\tilde{A}-\tilde{X}$ separation $\left(62 \mathrm{~cm}^{-1}\right)$.

${ }^{\text {c }}$ Redshift relative to the $\tilde{A}$-state vibrational frequency of the pumped non-CO-stretch mode $v_{\text {pump. }}$

${ }^{\mathrm{d}}$ Redshift relative to $\mathrm{n} v_{\mathrm{CO}}+v_{\text {pump. }}$. The relative wavenumber corresponds to the $\tilde{B}$-state CO stretch level.

${ }^{\mathrm{e}}$ Large uncertainty due to spectral congestion. 


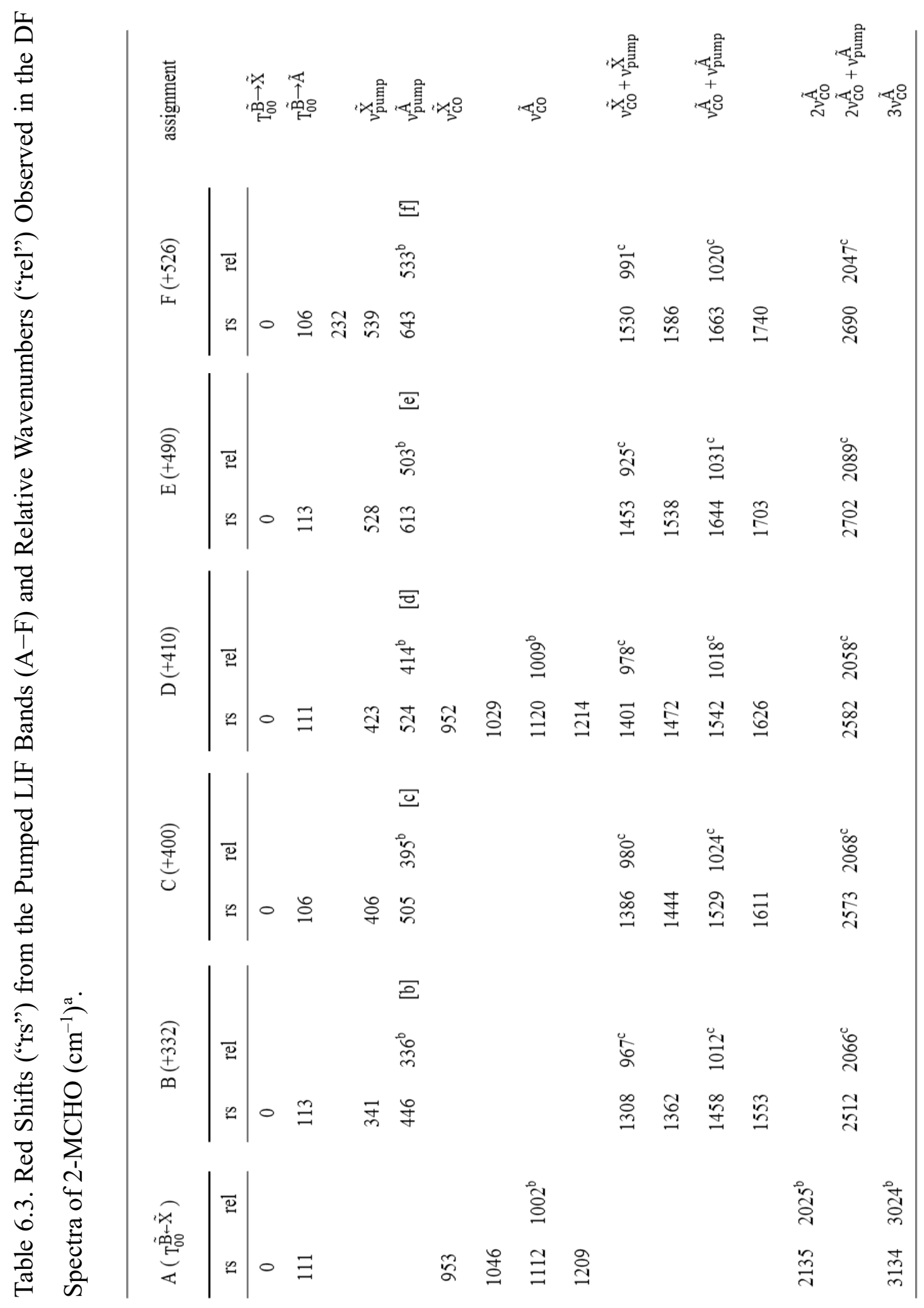


${ }^{a}$ Letters in square brackets correspond to labels in Figure 6.6.

${ }^{\mathrm{b}}$ Redshift relative to the averaged value of the $\tilde{A}-\tilde{X}$ separation $\left(110 \mathrm{~cm}^{-1}\right)$.

${ }^{\mathrm{c}}$ Redshift relative to the $\tilde{A}$-state vibrational frequency of the pumped mode $v_{\text {pump. }}$ 
Table 6.4. Calculated vibrational frequencies $\left(\mathrm{cm}^{-1}\right)$ of $\tilde{X}$ state 4-MCHO (with pseudoJahn-Teller distorted geometry) and Franck-Condon factors (FCFs) for transitions from the vibrational ground level of its $\tilde{B}$ state to $\tilde{X}$ state fundamental vibrational levels. (Threshold for FCFs is 0.001.)

\begin{tabular}{|c|c|c|}
\hline assignment & frequency & $\left|\left\langle\mathrm{v}^{\prime} \mid \mathrm{v}^{\prime \prime}\right\rangle\right|^{2}$ \\
\hline$v_{0}$ & 0 & 0.0059 \\
\hline$v_{57}$ & 109 & \\
\hline$v_{56}$ & 215 & \\
\hline$v_{55}$ & 234 & \\
\hline$v_{54}$ & 258 & \\
\hline$v_{53}$ & 276 & 0.0011 \\
\hline$v_{52}$ & 341 & \\
\hline$v_{51}$ & 368 & \\
\hline$v_{50}$ & 417 & \\
\hline$v_{49}$ & 445 & \\
\hline$v_{48}$ & 462 & \\
\hline$v_{47}$ & 584 & \\
\hline$v_{46}$ & 757 & 0.0013 \\
\hline$v_{45}$ & 792 & \\
\hline$v_{44}$ & 815 & 0.0015 \\
\hline$v_{43}$ & 880 & \\
\hline$v_{42}$ & 915 & 0.0016 \\
\hline$v_{41}$ & 938 & 0.0015 \\
\hline$v_{40}$ & 973 & \\
\hline$v_{39}$ & 984 & \\
\hline$v_{38}$ & 1019 & \\
\hline$v_{37}$ & 1039 & 0.0020 \\
\hline$v_{36}$ & 1067 & 0.0012 \\
\hline$v_{35}$ & 1073 & 0.0019 \\
\hline$v_{34^{a}}$ & 1122 & 0.0053 \\
\hline$v_{33}$ & 1140 & \\
\hline$v_{32}$ & 1175 & 0.0012 \\
\hline$v_{31}$ & 1240 & \\
\hline$v_{30}$ & 1252 & \\
\hline$v_{29}$ & 1271 & \\
\hline
\end{tabular}

Continued next page. 
Continued.

\begin{tabular}{|ccc|}
\hline assignment & frequency & $\left|\left\langle\mathrm{v}^{\prime} \mathrm{v}^{\prime \prime}\right\rangle\right|^{2}$ \\
\hline$v_{28}$ & 1294 & \\
$v_{27}$ & 1333 & \\
$v_{26}$ & 1340 & \\
$v_{25}$ & 1343 & \\
$v_{24}$ & 1351 & 0.0011 \\
$v_{23}$ & 1370 & \\
$v_{22}$ & 1391 & \\
$v_{21}$ & 1410 & \\
$v_{20}$ & 1434 \\
$v_{19}$ & 1499 \\
$v_{18}$ & 1506 \\
$v_{17}$ & 1511 \\
$v_{16}$ & 1520 \\
$v_{15}$ & 1521 \\
$v_{14}$ & 1522 \\
$v_{13}$ & 2930 \\
$v_{12}$ & 2992 \\
$v_{11}$ & 3011 \\
$v_{10}$ & 3020 \\
$v_{9}$ & 3033 \\
$v_{8}$ & 3040 \\
$v_{7}$ & 3053 \\
$v_{6}$ & 3062 \\
$v_{5}$ & 3069 \\
$v_{4}$ & 3086 \\
$v_{3}$ & 3094 \\
$v_{2}$ & 3099 \\
$v_{1}$ & 3115 \\
\hline
\end{tabular}

${ }^{a} \mathrm{CO}$ stretch 
Table 6.5. Calculated vibrational frequencies $\left(\mathrm{cm}^{-1}\right)$ of $\tilde{A}^{2} \mathrm{~A}^{\prime}$ state 4-MCHO and FranckCondon factors (FCFs) for transitions from the vibrational ground level of its $\tilde{B}^{2} \mathrm{~B}^{\prime}$ state to $\tilde{A}^{2} \mathrm{~A}^{\prime}$ state fundamental vibrational levels. (Threshold for FCFs is 0.001.)

\begin{tabular}{|c|c|c|c|}
\hline assignment & symmetry & frequency & $\left|\left\langle\mathrm{v}^{\prime} \mid \mathrm{v}^{\prime \prime}\right\rangle\right|^{2}$ \\
\hline$v_{0}$ & $a^{\prime}$ & 0 & 0.0065 \\
\hline$v_{32}$ & $a^{\prime}$ & 106 & \\
\hline$v_{57}$ & $a^{\prime \prime}$ & 206 & \\
\hline$v_{56}$ & $a^{\prime \prime}$ & 232 & \\
\hline$v_{31}$ & $a^{\prime}$ & 240 & 0.0014 \\
\hline$v_{55}$ & $a^{\prime \prime}$ & 256 & \\
\hline$v_{54}$ & $a^{\prime \prime}$ & 333 & \\
\hline$v_{30}$ & $a^{\prime}$ & 372 & \\
\hline$v_{29}$ & $a^{\prime}$ & 415 & 0.0015 \\
\hline$v_{53}$ & a" & 440 & \\
\hline$v_{28}$ & $a^{\prime}$ & 458 & 0.0030 \\
\hline$v_{27}$ & $a^{\prime}$ & 590 & \\
\hline$v_{26}$ & $a^{\prime}$ & 763 & \\
\hline$v_{52}$ & $a^{\prime \prime}$ & 782 & \\
\hline$v_{51}$ & $a^{\prime \prime}$ & 809 & \\
\hline$v_{25}$ & $a^{\prime}$ & 823 & \\
\hline$v_{50}$ & $a^{\prime \prime}$ & 899 & \\
\hline$v_{24}$ & $a^{\prime}$ & 951 & 0.0023 \\
\hline$v_{49}$ & $a^{\prime \prime}$ & 966 & \\
\hline$v_{23}$ & $a^{\prime}$ & 969 & 0.0011 \\
\hline$v_{22}$ & $a^{\prime}$ & 1023 & \\
\hline$v_{48}$ & a" & 1038 & \\
\hline$v_{21}{ }^{a}$ & $a^{\prime}$ & 1060 & 0.0122 \\
\hline$v_{47}$ & $a^{\prime \prime}$ & 1079 & \\
\hline$v_{20}$ & $a^{\prime}$ & 1087 & \\
\hline$v_{46}$ & $a^{\prime \prime}$ & 1135 & \\
\hline$v_{19}$ & $a^{\prime}$ & 1171 & 0.0030 \\
\hline$v_{45}$ & a" & 1204 & \\
\hline$v_{18}$ & $a^{\prime}$ & 1220 & 0.0018 \\
\hline$v_{44}$ & $a^{\prime \prime}$ & 1248 & \\
\hline
\end{tabular}

Continued next page. 
Continued.

\begin{tabular}{|c|c|c|c|}
\hline assignment & symmetry & frequency & $\left|\left\langle\mathrm{v}^{\prime} \mid \mathrm{v}^{\prime \prime}\right\rangle\right|^{2}$ \\
\hline$v_{17}$ & $a^{\prime}$ & 1279 & \\
\hline$v_{16}$ & $a^{\prime}$ & 1297 & 0.0013 \\
\hline$v_{15}$ & $a^{\prime}$ & 1342 & \\
\hline$v_{43}$ & a" & 1342 & \\
\hline$v_{42}$ & a" & 1347 & \\
\hline$v_{14}$ & $a^{\prime}$ & 1377 & \\
\hline$v_{41}$ & a" & 1388 & \\
\hline$v_{13}$ & $\mathrm{a}^{\prime}$ & 1408 & \\
\hline$v_{12}$ & $\mathrm{a}^{\prime}$ & 1430 & \\
\hline$v_{40}$ & $\mathrm{a}^{\prime \prime}$ & 1499 & \\
\hline$v_{39}$ & $a^{\prime \prime}$ & 1503 & \\
\hline$v_{11}$ & $\mathrm{a}^{\prime}$ & 1511 & \\
\hline$v_{10}$ & $\mathrm{a}^{\prime}$ & 1519 & \\
\hline$v_{38}$ & a" & 1521 & \\
\hline$v_{9}$ & $\mathrm{a}^{\prime}$ & 1522 & \\
\hline$v_{8}$ & $a^{\prime}$ & 2837 & \\
\hline$v_{7}$ & $\mathrm{a}^{\prime}$ & 2989 & \\
\hline$v_{37}$ & a" & 3003 & \\
\hline$v_{6}$ & $a^{\prime}$ & 3011 & \\
\hline$v_{5}$ & $a^{\prime}$ & 3032 & \\
\hline$v_{36}$ & $\mathrm{a}^{\prime \prime}$ & 3033 & \\
\hline$v_{4}$ & $\mathrm{a}^{\prime}$ & 3038 & \\
\hline$v_{35}$ & $\mathrm{a}^{\prime \prime}$ & 3059 & \\
\hline$v_{3}$ & $\mathrm{a}^{\prime}$ & 3063 & \\
\hline$v_{34}$ & a" & 3089 & \\
\hline$v_{2}$ & $\mathrm{a}^{\prime}$ & 3091 & \\
\hline$v_{1}$ & $a^{\prime}$ & 3093 & \\
\hline$v_{33}$ & a" & 3099 & \\
\hline
\end{tabular}

${ }^{\mathrm{a}} \mathrm{CO}$ stretch 
Table 6.6. Calculated vibrational frequencies $\left(\right.$ in $\mathrm{cm}^{-1}$ ) of $\tilde{X}$ state 3-MCHO and FranckCondon factors (FCFs) for transitions from the vibrational ground level of its $\tilde{B}$ state to $\tilde{X}$ state fundamental vibrational levels. (Threshold for FCFs is 0.001.)

\begin{tabular}{|c|c|c|}
\hline assignment & frequency & $\left|\left\langle\mathrm{v}^{\prime} \mid \mathrm{v}^{\prime \prime}\right\rangle\right|^{2}$ \\
\hline$v_{0}$ & 0 & 0.0051 \\
\hline$v_{57}$ & 146 & \\
\hline$v_{56}$ & 162 & \\
\hline$v_{55}$ & 232 & \\
\hline$v_{54}$ & 256 & 0.0010 \\
\hline$v_{53}$ & 287 & \\
\hline$v_{52}$ & 358 & 0.0020 \\
\hline$v_{51}$ & 407 & \\
\hline$v_{50}$ & 416 & \\
\hline$v_{49}$ & 431 & \\
\hline$v_{48}$ & 467 & \\
\hline$v_{47}$ & 545 & \\
\hline$v_{46}$ & 778 & \\
\hline$v_{45}$ & 820 & 0.0018 \\
\hline$v_{44}$ & 852 & 0.0015 \\
\hline$v_{43}$ & 873 & \\
\hline$v_{42}$ & 886 & \\
\hline$v_{41}$ & 938 & \\
\hline$v_{40}$ & 962 & 0.0013 \\
\hline$v_{39}$ & 981 & \\
\hline$v_{38}$ & 1014 & 0.0015 \\
\hline$v_{37}$ & 1048 & 0.0015 \\
\hline$v_{36}$ & 1076 & \\
\hline$v_{35}$ & 1086 & \\
\hline$v_{3} 4^{a}$ & 1120 & 0.0038 \\
\hline$v_{33}$ & 1143 & \\
\hline$v_{32}$ & 1160 & \\
\hline$v_{31}$ & 1236 & \\
\hline$v_{30}$ & 1264 & \\
\hline$v_{29}$ & 1280 & \\
\hline
\end{tabular}

Continued next page. 
Continued.

\begin{tabular}{|c|c|c|}
\hline assignment & frequency & $\left|\left\langle\mathrm{v}^{\mathrm{v}} \mid \mathrm{v}^{\prime \prime}\right\rangle\right|^{2}$ \\
\hline$v_{28}$ & 1295 & \\
\hline$v_{27}$ & 1306 & \\
\hline$v_{26}$ & 1342 & \\
\hline$v_{25}$ & 1348 & \\
\hline$v_{24}$ & 1370 & \\
\hline$v_{23}$ & 1380 & \\
\hline$v_{22}$ & 1387 & \\
\hline$v_{21}$ & 1401 & \\
\hline$v_{20}$ & 1430 & \\
\hline$v_{19}$ & 1502 & \\
\hline$v_{18}$ & 1505 & \\
\hline$v_{17}$ & 1512 & \\
\hline$v_{16}$ & 1519 & \\
\hline$v_{15}$ & 1521 & \\
\hline$v_{14}$ & 1524 & \\
\hline$v_{13}$ & 2930 & \\
\hline$v_{12}$ & 3002 & \\
\hline$v_{11}$ & 3014 & \\
\hline$v_{10}$ & 3021 & \\
\hline$v_{9}$ & 3034 & \\
\hline$v_{8}$ & 3040 & \\
\hline$v_{7}$ & 3054 & \\
\hline$v_{6}$ & 3062 & \\
\hline$v_{5}$ & 3071 & \\
\hline$v_{4}$ & 3086 & \\
\hline$v_{3}$ & 3097 & \\
\hline$v_{2}$ & 3102 & \\
\hline$v_{1}$ & 3109 & \\
\hline
\end{tabular}

${ }^{\mathrm{a}} \mathrm{CO}$ stretch 
Table 6.7. Calculated vibrational frequencies $\left(\mathrm{cm}^{-1}\right)$ of $\tilde{X}$ state 2-MCHO and FranckCondon factors (FCFs) for transitions from the vibrational ground level of its $\tilde{B}$ state to $\tilde{X}$ state fundamental vibrational levels. (Threshold for FCFs is 0.001.)

\begin{tabular}{|c|c|c|}
\hline assignment & frequency & $\left|\left\langle\mathrm{v}^{\prime} \mid \mathrm{v}^{\prime \prime}\right\rangle\right|^{2}$ \\
\hline$v_{0}$ & 0 & 0.0055 \\
\hline$v_{57}$ & 116 & \\
\hline$v_{56}$ & 182 & \\
\hline$v_{55}$ & 229 & \\
\hline$v_{54}$ & 259 & 0.0011 \\
\hline$v_{53}$ & 319 & \\
\hline$v_{52}$ & 342 & \\
\hline$v_{51}$ & 348 & 0.0018 \\
\hline$v_{50}$ & 425 & \\
\hline$v_{49}$ & 431 & \\
\hline$v_{48}$ & 486 & \\
\hline$v_{47}$ & 555 & \\
\hline$v_{46}$ & 760 & 0.0011 \\
\hline$v_{45}$ & 827 & 0.0015 \\
\hline$v_{44}$ & 837 & \\
\hline$v_{43}$ & 857 & \\
\hline$v_{42}$ & 900 & 0.0029 \\
\hline$v_{41}$ & 936 & 0.0011 \\
\hline$v_{40}$ & 951 & 0.0019 \\
\hline$v_{39}$ & 991 & \\
\hline$v_{38}$ & 998 & \\
\hline$v_{37}$ & 1064 & 0.0011 \\
\hline$v_{36}$ & 1080 & \\
\hline$v_{35}$ & 1083 & \\
\hline$v_{34^{\mathrm{a}}}$ & 1123 & 0.0048 \\
\hline$v_{33}$ & 1138 & 0.0014 \\
\hline$v_{32}$ & 1171 & \\
\hline$v_{31}$ & 1217 & \\
\hline$v_{30}$ & 1263 & \\
\hline$v_{20}$ & 1268 & \\
\hline
\end{tabular}

Continued next page. 
Continued.

\begin{tabular}{|c|c|c|}
\hline assignment & frequency & $\left|\left\langle\mathrm{v}^{\prime} \mid \mathrm{v}^{\prime \prime}\right\rangle\right|^{2}$ \\
\hline$v_{28}$ & 1286 & \\
\hline$v_{27}$ & 1325 & \\
\hline$v_{26}$ & 1338 & \\
\hline$v_{25}$ & 1348 & \\
\hline$v_{24}$ & 1374 & \\
\hline$v_{23}$ & 1375 & \\
\hline$v_{22}$ & 1390 & \\
\hline$v_{21}$ & 1396 & \\
\hline$v_{20}$ & 1429 & \\
\hline$v_{19}$ & 1502 & \\
\hline$v_{18}$ & 1507 & \\
\hline$v_{17}$ & 1511 & \\
\hline$v_{16}$ & 1519 & \\
\hline$v_{15}$ & 1522 & \\
\hline$v_{14}$ & 1523 & \\
\hline$v_{13}$ & 2917 & \\
\hline$v_{12}$ & 3007 & \\
\hline$v_{11}$ & 3018 & \\
\hline$v_{10}$ & 3023 & \\
\hline$v_{9}$ & 3028 & \\
\hline$v_{8}$ & 3034 & \\
\hline$v_{7}$ & 3056 & \\
\hline$v_{6}$ & 3061 & \\
\hline$v_{5}$ & 3068 & \\
\hline$v_{4}$ & 3075 & \\
\hline$v_{3}$ & 3094 & \\
\hline$v_{2}$ & 3114 & \\
\hline$v_{1}$ & 3116 & \\
\hline
\end{tabular}

${ }^{\mathrm{a}} \mathrm{CO}$ stretch 


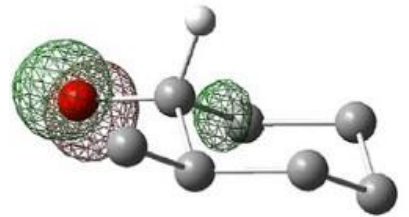

(a)
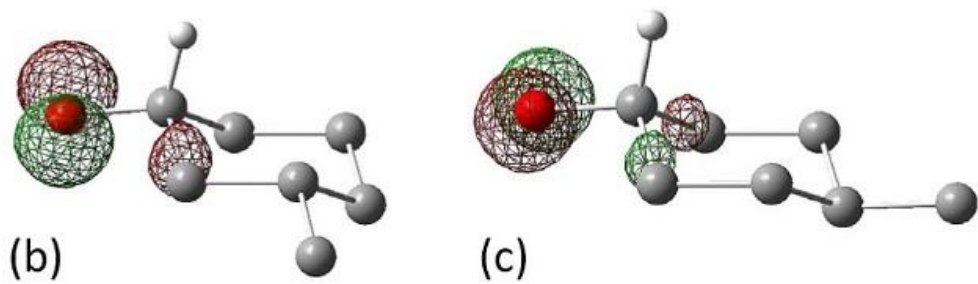

(c)

Figure 6.1. Half-filled orbitals of the lowest-energy conformers of (a) 2-MCHO, (b) 3$\mathrm{MCHO}$, and (c) 4-MCHO. Hydrogen atoms are not shown for clarity except the one at the $\mathrm{C}_{1}$-position. 


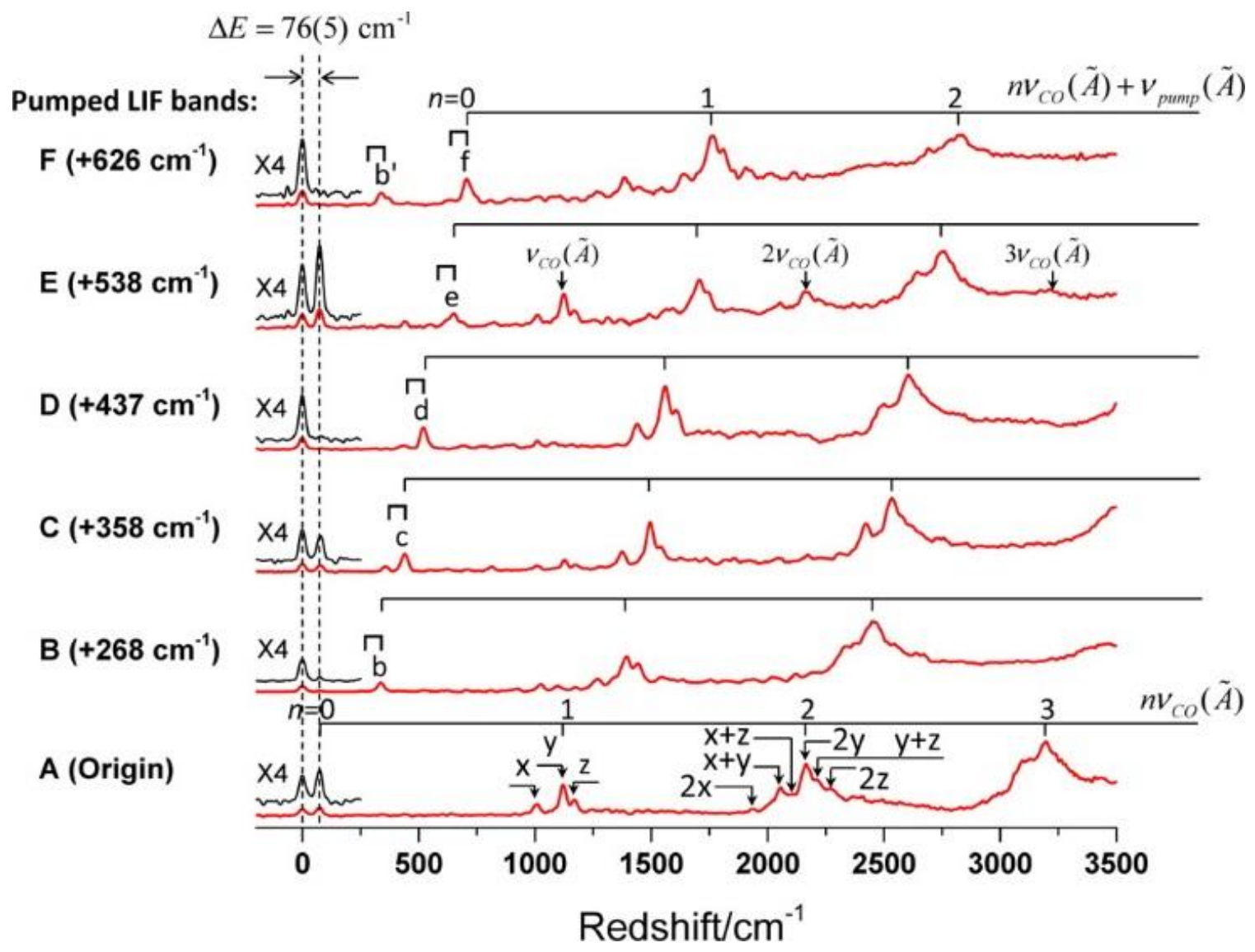

Figure 6.2. DF spectra of 4-MCHO. Numbers in parentheses after the LIF bands are relative wavenumbers with respect to the origin band (band A) at $26906 \mathrm{~cm}^{-1}$. 


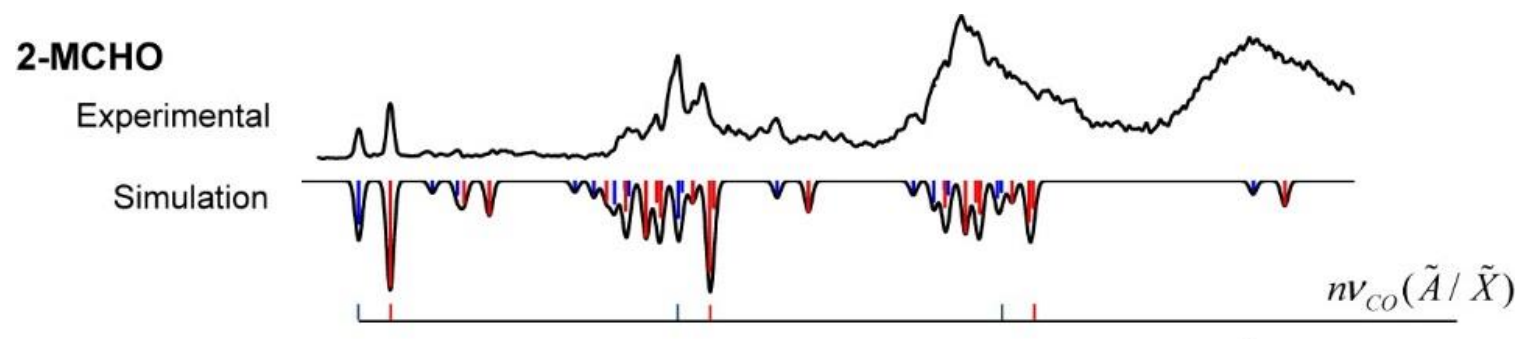

3-MCHO

Experimental

Simulation

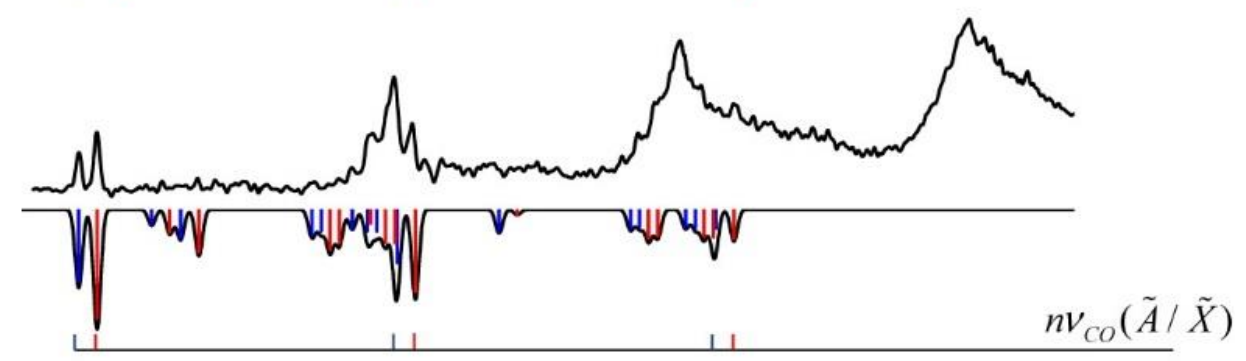

\section{4-MCHO}

Experimental

Simulation

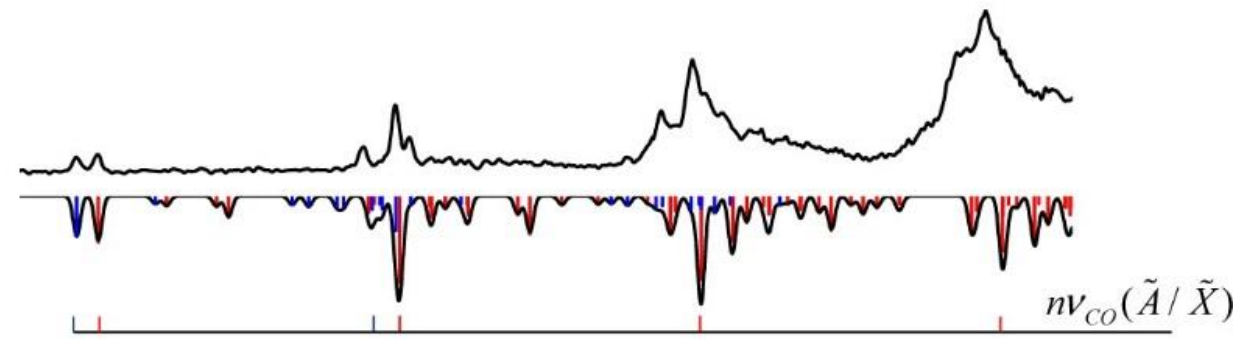

Figure 6.3. DF spectra of 2, 3-, and 4-MCHOs by pumping the LIF origin bands compared with calculated transition intensities and simulated spectra. Blue and red sticks represent $\tilde{B} \rightarrow \tilde{X}$ and $\tilde{B} \rightarrow \tilde{A}$ transitions, respectively. 


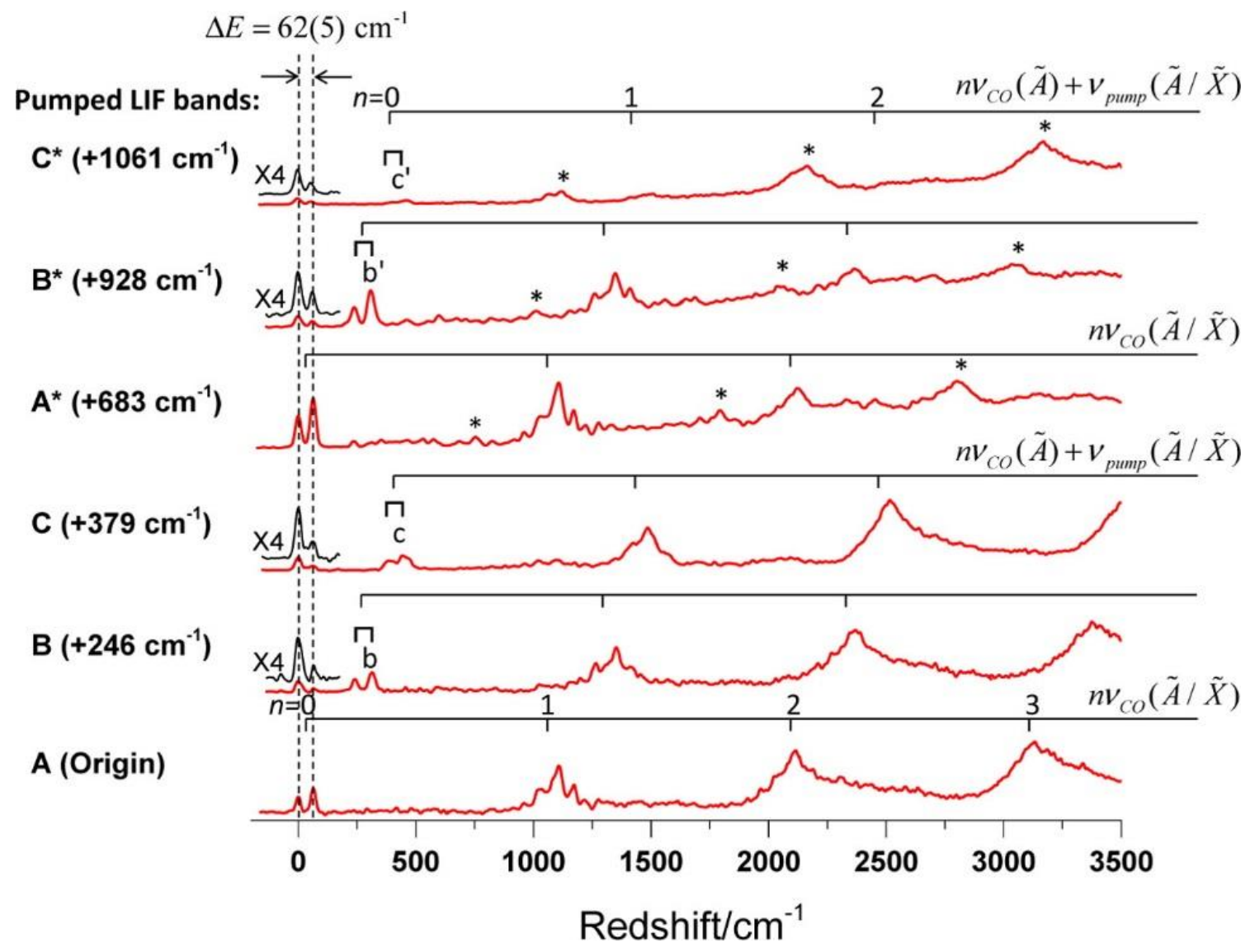

Figure 6.4. DF spectra of 3-MCHO. Numbers in parentheses after the LIF bands are relative wavenumbers with respect to the origin band (band A) at $26677 \mathrm{~cm}^{-1}$. 


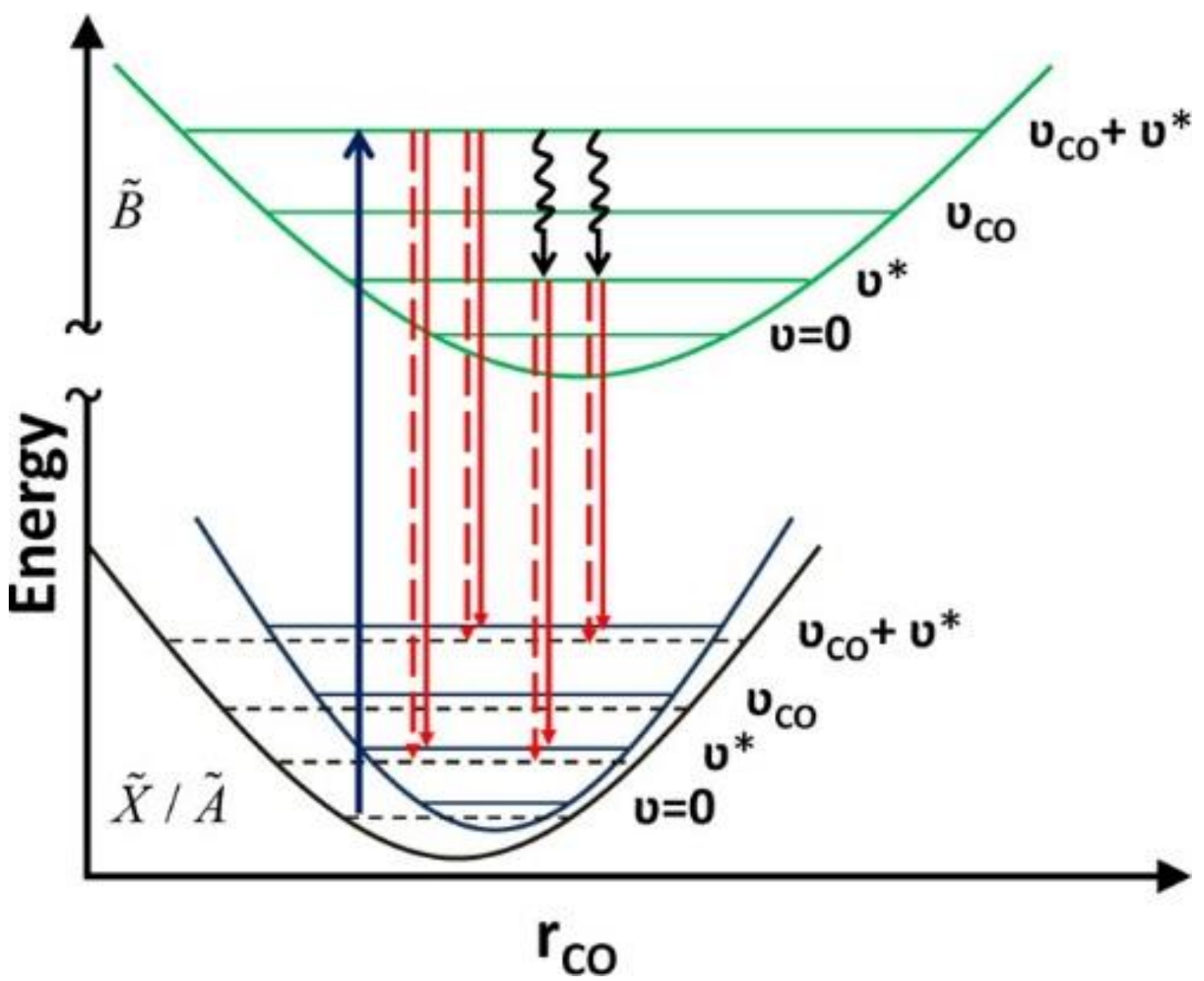

Figure 6.5. Excited-state vibrational population relaxation (wavy arrows) and fluorescence emission (red arrows) after $\tilde{\mathrm{B}} \leftarrow \tilde{X}$ excitation (black arrow) of 3-MCHO to CO-stretch combinations bands $\left(v_{\mathrm{CO}}+v^{*}\right)$. $\tilde{X}$ and $\tilde{A}$ states are semi degenerate. Dashed horizontal lines represent vibrational energy levels of the $\tilde{X}$ state. 


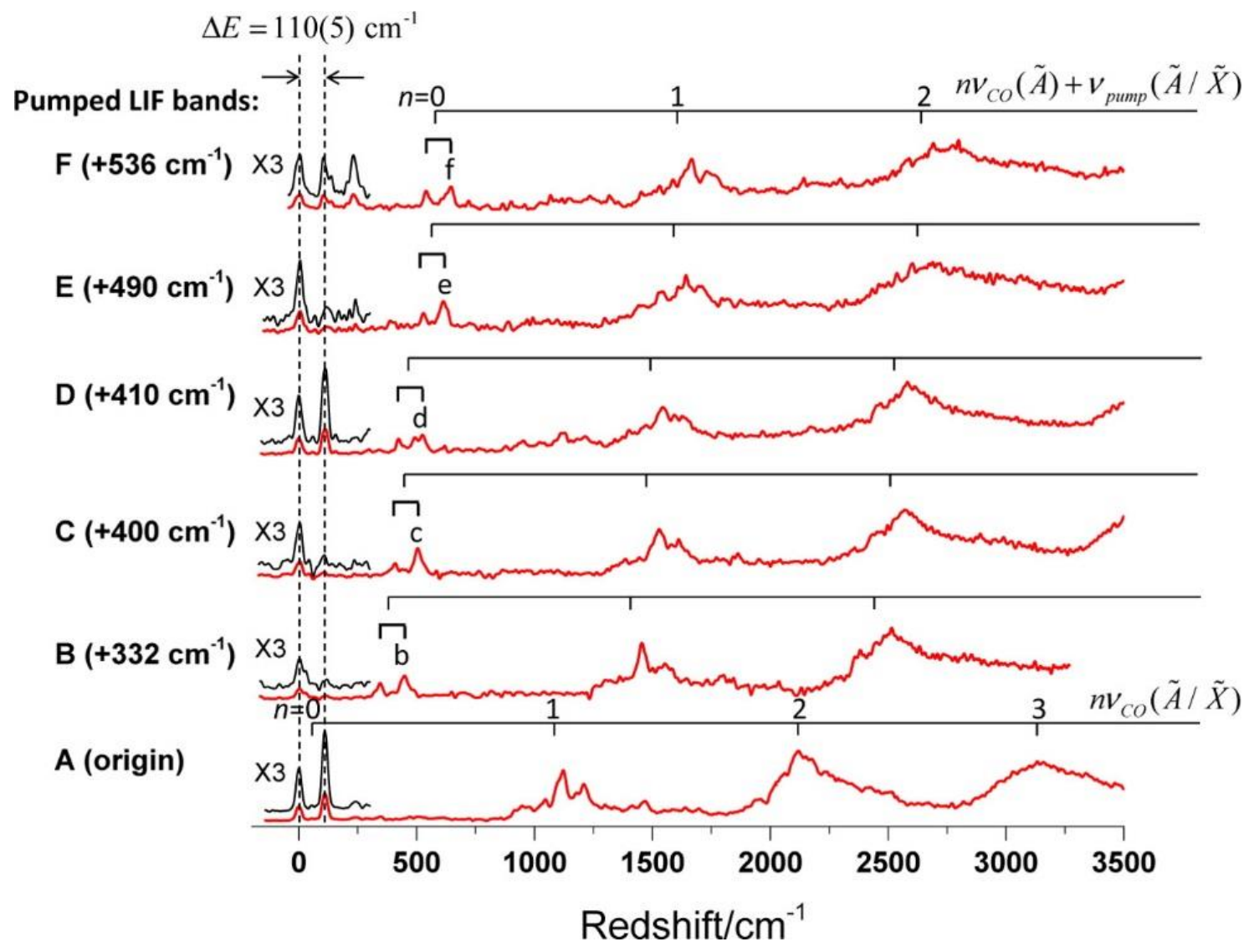

Figure 6.6. DF spectra of 2-MCHO. Numbers in parentheses after the LIF bands are relative wavenumbers with respect to the origin band (band A) at $26275 \mathrm{~cm}^{-1}$. 


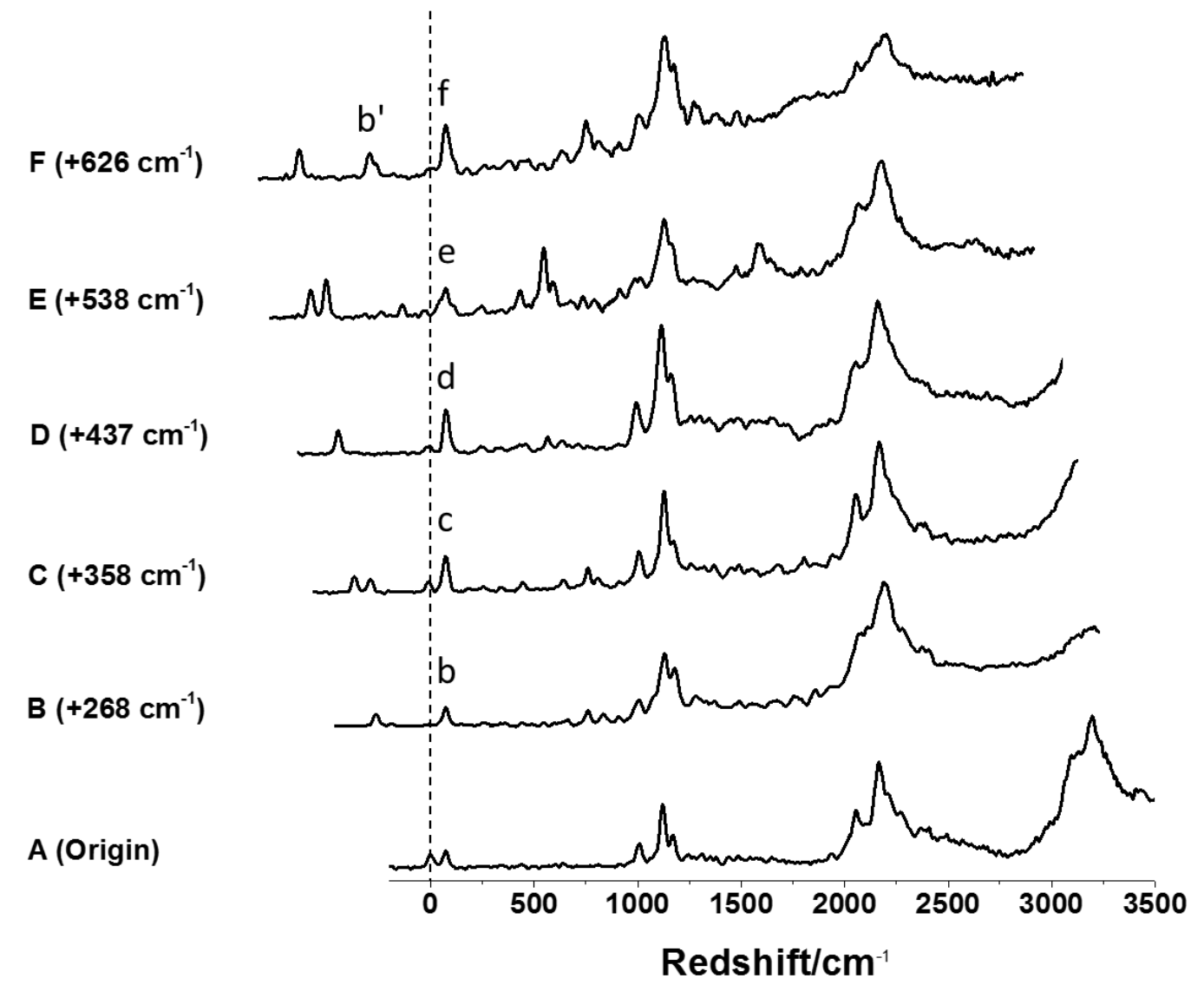

Figure 6.7. DF spectra of 4-MCHO. The spectra by pumping vibronic bands other than the origin band (A) in the LIF spectrum are blueshifted by the A-state vibrational frequency of the pumped mode so that the DF bands to the $\tilde{A}$-state vibrational level of the pumped modes are aligned. 


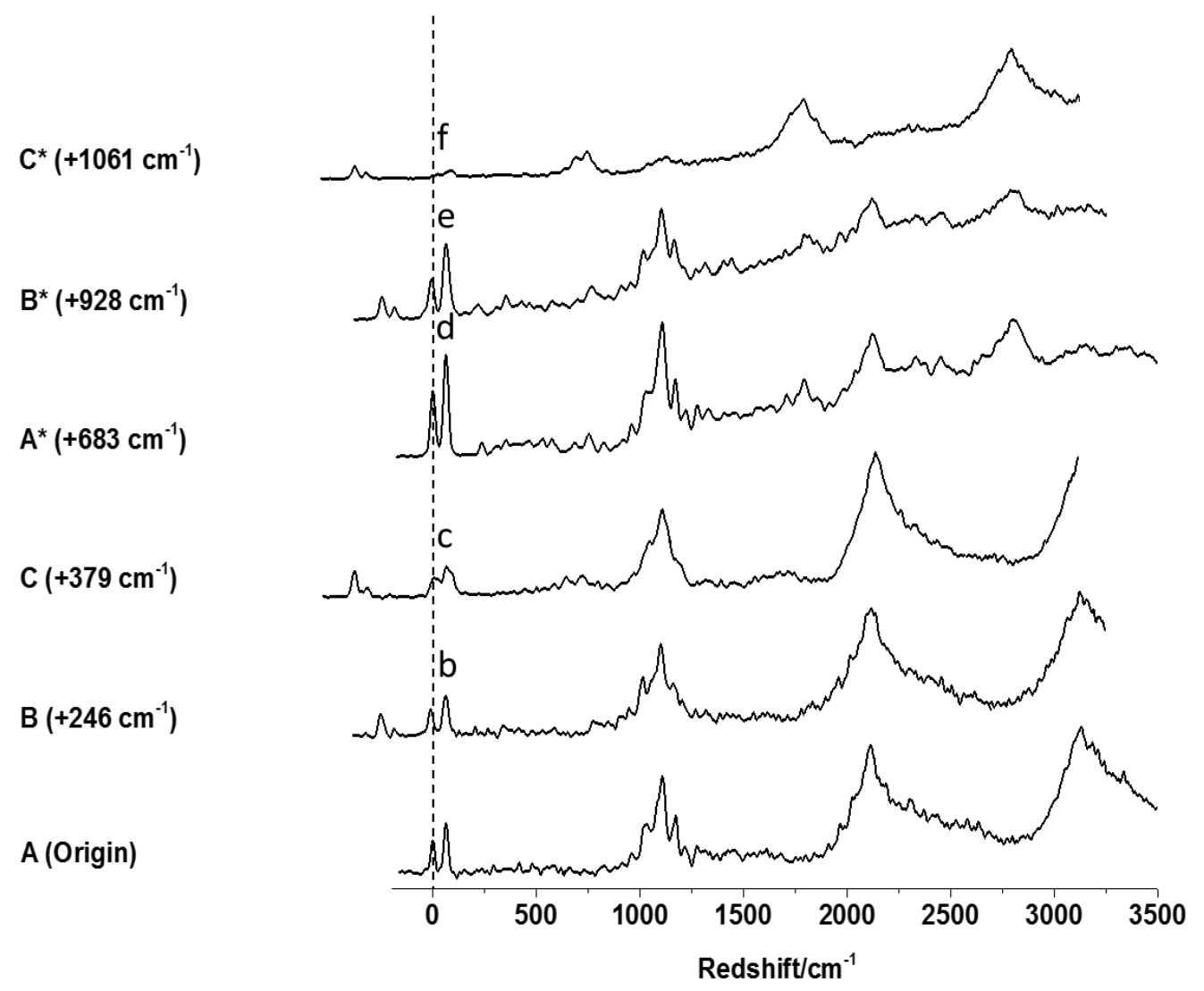

Figure 6.8. DF spectra of 3-MCHO. The spectra by pumping vibronic bands other than the origin band (A) and the CO stretch band (A*) in the LIF spectrum are blueshifted by the A-state vibrational frequency of the pumped mode so that the DF bands to the $\tilde{A}$-state vibrational level of the pumped modes are aligned. 
$\mathrm{F}\left(+536 \mathrm{~cm}^{-1}\right)$

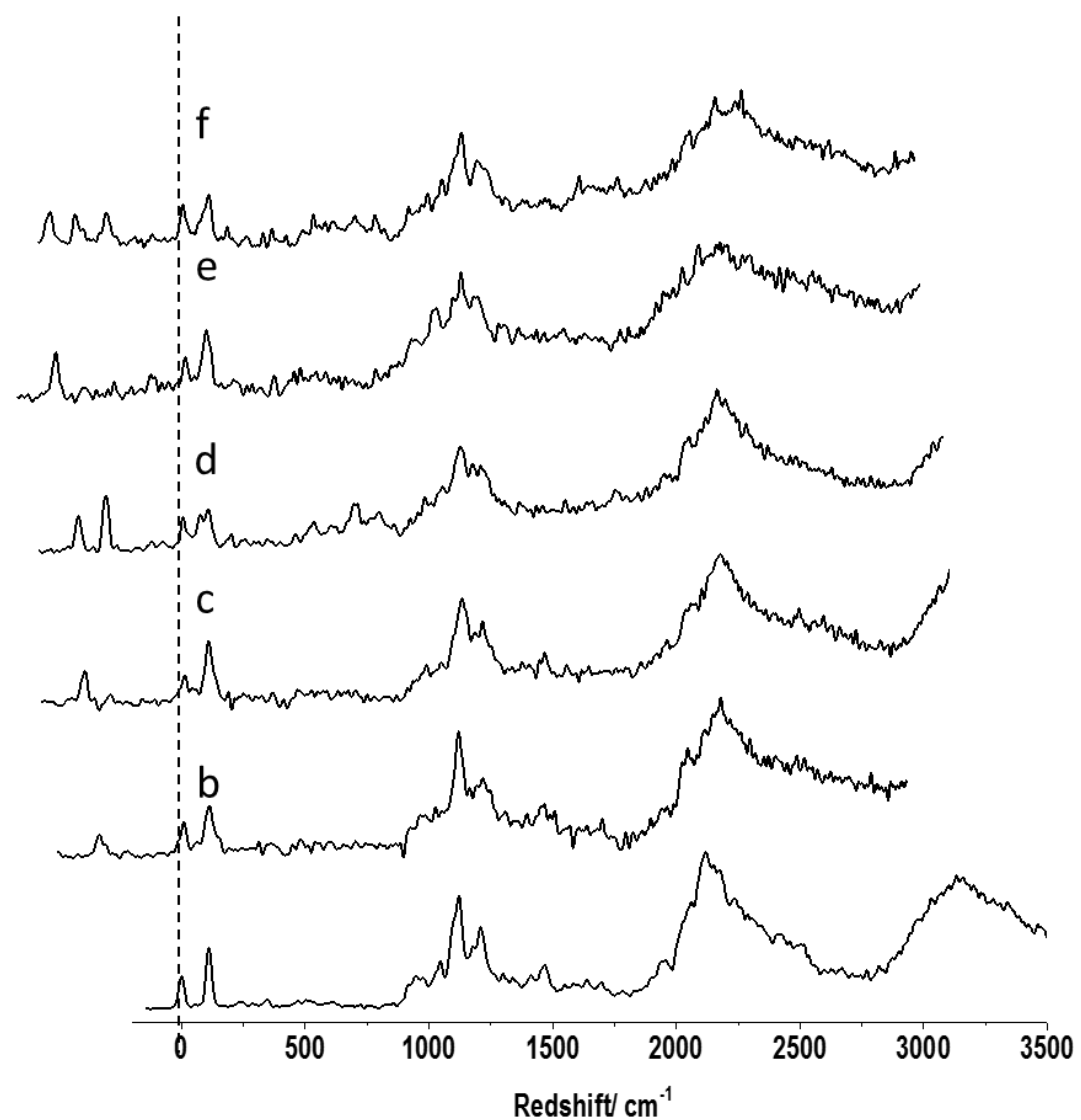

Figure 6.9. DF spectra of 2-MCHO. The spectra by pumping vibronic bands other than the origin band (A) in the LIF spectrum are blueshifted by the A-state vibrational frequency of the pumped mode so that the DF bands to the $\tilde{A}$-state vibrational level of the pumped modes are aligned. 


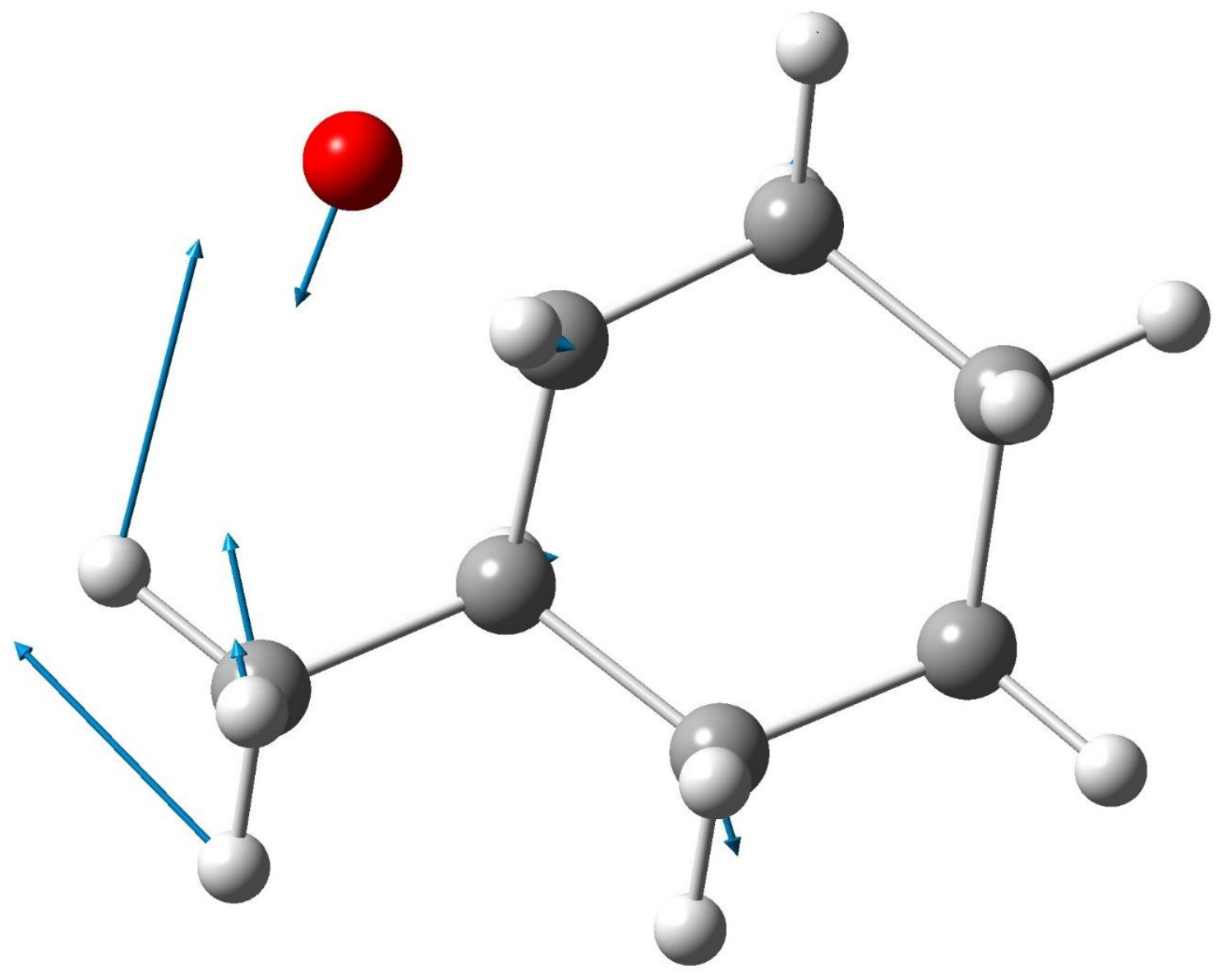

Figure 6.10. Figure: B-state vibrational mode $v_{54}$ of 2-MCHO. 


\section{CHAPTER VII}

\section{DISPERSED FLUORESCENCE SPECTROSCOPY OF JET-COOLED 1- METHYLCYCLOHEXOXY RADICALS}

\subsection{Introduction}

The cyclohexoxy radical and its methyl substitutions are important reaction intermediates in combustion and atmospheric chemistry. ${ }^{148,149}$ Spectroscopic investigations can serve as diagnostics of these free radicals in kinetic studies, ${ }^{36,37,150,151}$ and provide information about their structure and dynamics. Previously, laser-induced fluorescence (LIF) ${ }^{37}$ and dispersed fluorescence (DF) ${ }^{93}$ spectra of 2-, 3-, and 4-methylcyclohexoxy (MCHO) radicals have been obtained under jet-cooled conditions. Spectral analysis and assignment helped determination of the vibronic structure of these isomers and understanding of the effect of methyl substitution. Furthermore, the LIF spectrum of 1-MCHO was reported by Wu et al. ${ }^{37}$ Significant differences between LIF spectra of the four positional isomers of MCHOs have been found. For instance, strong $\mathrm{CO}$-stretch progressions are present in the LIF spectra of the 4- and 3-MCHO radicals but not in those of 2- and 1-MCHOs. Within all four positional isomers, 1-MCHO is unique due to its tertiary structure and the juxtaposition of the methyl group to the $\mathrm{CO}$ bond. In addition, two lowest-energy conformers, chair-axial, and chair-equatorial have been observed in the LIF spectra under 
jet-cooled conditions, which further complicates the spectrum. In the present letter, we report the DF spectra of 1-MCHO under jet-cooled conditions. They are significantly different from those of 2-, 3-, and 4-MCHOs, which are similar to one another.

\subsection{Quantum Chemical Calculations}

1-MCHO radicals, there are two types of conformers, namely, chair conformers and twisted-boat conformers. Calculations predicted the energy barriers from the twisted-boat to the chair conformers are about $3.05-4.96 \mathrm{kcal} / \mathrm{mol}$, while the barriers from chair conformers to twisted-boat conformers are $8.62-10.43 \mathrm{kcal} / \mathrm{mol}$. Thus, twisted-boat conformers are easy to convert to chair conformers. ${ }^{37}$ The lowest energy conformer of 1MCHO is a chair-axial conformer in which the oxygen atom is located at the axial orientation, while the methyl group is at the equatorial orientation. One the other hand chair-equatorial conformer in which oxygen atom at the equatorial position and the methyl group at the axial orientation and is $1.60 \mathrm{kcal} / \mathrm{mol}$ higher in energy than the chair-axial conformer. ${ }^{37}$ Both chair-axial and chair-equatorial conformers of 1-MCHO radical have $C_{s}$ symmetry. Previously, extensive calculations were performed on the $\tilde{X}$ - and $\tilde{B}$-state 1MCHO radicals and used to guide the analysis of the experimentally obtained LIF spectra. ${ }^{37}$ Such analysis allowed the assignment of vibronic bands in the LIF spectra and suggests that all LIF bands observed under jet-cooled conditions are due to the lowestenergy conformers. In the present work, two types of new calculations have been carried out in aid of the analysis of the DF spectra. First, density functional theory (DFT) 
calculations at the UCAM-B3LYP/6-311+G(d,p $)^{112}$ level of theory were performed to predict the energy separation between the $\tilde{X}$ and the $\tilde{A}$ states of two conformers of 1$\mathrm{MCHO}$, which retains the $C_{s}$ symmetry of the 1-MCHO radical. This was done by putting the unpaired electron in either $p_{x}$ or $p_{y}$ orbitals localized on the oxygen atom. The calculations predict that the ground electronic state $(\tilde{X})$ has A" symmetry whereas the first excited state $(\tilde{A})$ is of $\mathrm{A}^{\prime}$ symmetry. The energy separation between these two states of chair axial and chair equatorial conformers were found to be $286 \mathrm{~cm}^{-1}$ and $206 \mathrm{~cm}^{-1}$ (with ZPE corrections). When the molecular symmetry was allowed to be lowered to $C_{l}$ for both conformers, geometry optimization resulted in the pJT-distorted structure of the 1-MCHO conformers. The pJT stabilization energy, defined as the energy difference between the ground-state $\left(\tilde{X}^{2} \mathrm{~A}^{\prime \prime}\right)$ molecule with $C_{s}$ symmetry and that with $C_{l}$ geometry, i.e., no symmetry, was found to be $174 \mathrm{~cm}^{-1}$ for chair axial and $151 \mathrm{~cm}^{-1}$ for chair equatorial conformer.

Calculated relative energies of 1-MCHO conformers, energy separation between $\tilde{A}$-and $\tilde{X}$-states $\left(\Delta E^{\tilde{A}-\tilde{X}}\right)$ and $\tilde{X}$ - and $\tilde{A}$-state $\mathrm{CO}$-stretch and $\mathrm{C}-\mathrm{CH}_{3}$-stretch frequencies of 1-MCHO conformers are listed in Table 7.1.

\subsection{Results}

Selected vibronic bands in the LIF spectrum of 1-MCHO were pumped, and the DF spectra were obtained. The LIF bands are labeled by capital letters following ref. ${ }^{37}$ DF spectra obtained by pumping eleven bands (B, D, E, F, G, H, I, J, K, L, N) are presented in the 
letter. DF spectra pumping most of the other bands are either structureless or of low signalto-noise ratio, which hinders definitive assignment of the transitions. Band A and Band C in the LIF spectrum are different from other DF spectra obtained. It is therefore attributed to an unknown impurity or some other molecular transitions. DF spectrum pumping band, A and band $\mathrm{C}$, are given in Figure 7.5.

\subsubsection{Chair-Axial Conformer}

DF spectra obtained by pumping eight vibronic bands (D, F, H, I, J, K, L, N) that belong to the chair-axial conformer are compared in Figure 7.1. In the DF spectrum pumping Band $\mathrm{D}$, the LIF origin band, the first peak other than the zero-redshift one is at $238 \mathrm{~cm}^{-1}$. This peak is hence assigned to the $\tilde{B} \rightarrow \tilde{A}$ origin band, corresponding to an $\tilde{A}-\tilde{X}$ separation of $238(5) \mathrm{cm}^{-1}$ for the chair-axial conformer. Quantum chemical calculations predicted a large ( $35 \mathrm{~m} \AA)$ decrease in $\mathrm{C}^{-\mathrm{CH}_{3}}$ bond length upon $\tilde{B} \leftarrow \tilde{A} / \tilde{X}$ excitation.5 Large FranckCondon factors are therefore expected for transitions from the vibrational ground level of the $\tilde{B}$ state to the $\mathrm{C}-\mathrm{CH}_{3}$ stretch levels of the $\tilde{A} / \tilde{X}$ state. It is also the first member of a vibrational progression with separations of $\sim 486 \mathrm{~cm}^{-1}$ (see figure 7.2). It is therefore assigned to the transition to the first $\mathrm{C}-\mathrm{CH}_{3}$ stretch level of the $\tilde{X}$ state. The second member of the aforementioned progression is at $517 \mathrm{~cm}^{-1}, 755 \mathrm{~cm}^{-1}$ above the $\tilde{B} \rightarrow \tilde{A}$ origin band. Hence, this transition is assigned to the $\mathrm{C}-\mathrm{CH}_{3}$ stretch level of the $\tilde{A}$ state. As a comparison density functional theory (DFT) calculations at the UCAM-B3LYP/6-311+G(d) level of 
theory predicts fundamental frequencies of $553 \mathrm{~cm}^{-1}$ and $569 \mathrm{~cm}^{-1}$ for the $\tilde{X}$ - state and $\tilde{A}$ state $\mathrm{C}-\mathrm{CH}_{3}$ stretch modes, respectively.

The peak at $996 \mathrm{~cm}^{-1}$ is assigned to the first overtone of the $\mathrm{C}_{-} \mathrm{CH}_{3}$ stretch of the $\tilde{X}$ -state. The peak at $1267 \mathrm{~cm}^{-1}$ is assigned to the first overtone of the $\mathrm{C}-\mathrm{CH}_{3}$ stretch of the $\tilde{A}$-state. In both $\tilde{X}$ - and $\tilde{A}$-state transition, we did not observe the CO-stretch transition. Quantum calculation predicted the CO stretch frequencies $925 \mathrm{~cm}^{-1}$ and $919 \mathrm{~cm}^{-1}$ for $\tilde{X}$ and $\tilde{A}$-state assigned to the transition to the $\tilde{A}$-state CO-stretch level. But in the DF spectrum, we did not observe any peak that can be assigned CO-stretch level. The strong peak at $1578 \mathrm{~cm}^{-1}$ can be assigned to the combination of $\mathrm{CO}$ stretch and $\mathrm{C}-\mathrm{CH}_{3}$ mode.

The strong peaks in the higher-frequency region contain multiple, partially resolved transitions. Transition frequencies were determined in multi-peak fitting to Gaussian shapes. Transition frequencies are summarized in Table 7.2. Three vibrational progressions are determined: $v_{\mathrm{C}-\mathrm{CH} 3}(\tilde{X}), v_{\mathrm{C}-\mathrm{CH} 3}(\tilde{A})$, and $(\mathrm{n}-3) v_{\mathrm{C}-\mathrm{CH} 3}(\tilde{X})+v_{\mathrm{CO}}(\tilde{X})$ with $\mathrm{n}=4-10$ (Figure 7.2). The constant intervals between the progression members suggest the absence of any detectable anharmonicity or Fermi resonance. Although transitions to the fundamental levels of the $\tilde{X}$ - and $\tilde{A}$-state $\mathrm{CO}$ stretch modes were not observed, their vibrational frequencies were determined by vibrational combination differences to be 486 $\mathrm{cm}^{-1}$ and $1005 \mathrm{~cm}^{-1}$, respectively.

In the DF spectra of 2-, 3-, and 4-MCHO radicals, transitions to high-energy COstretch levels often have broad linewidth and are obscured by background signal due to interaction between highly excited vibrational levels. In the present work of 1-MCHO, 
sharp $\mathrm{C}-\mathrm{CH}_{3}$ stretch transitions were observed for $\tilde{X}$ - and $\tilde{A}$-state. In addition, the intensities of the DF peaks oscillate when the vibrational quantum numbers increase. Explanation of these observations based computational results and theoretical analysis are given in Section 7.4

There is a noticeable difference when the origin (band D) band in the LIF spectrum was pumped, only four strong and resolved peaks $\left(0 \mathrm{~cm}^{-1}, 1578 \mathrm{~cm}^{-1}, 3126 \mathrm{~cm}^{-1}\right.$, and 4628 $\mathrm{cm}^{-1}$ ) were observed in the DF spectrum. These peaks are of equal spacing and are assigned to transitions to the vibrational ground level, the $v_{\mathrm{C}-\mathrm{CH} 3}+v_{\mathrm{CO}}$ level, the $4 v_{\mathrm{C}-\mathrm{CH} 3}+v_{\mathrm{CO}}$ level, and the $7 v_{\mathrm{C}-\mathrm{CH} 3}+v_{\mathrm{CO}}$ of the $\tilde{X}$ state. A possible mechanism for the difference in transition intensities is discussed in Section 7.4.

When the band $\mathrm{N}$ in the LIF spectrum was pumped, the DF structure of the band same as the origin band (band $\mathrm{D}$ ). Hence band $\mathrm{N}$ can be assigned to the transition of $\mathrm{CO}$ stretch level of $\tilde{B}$ state.

\subsubsection{Chair-Equatorial Conformer}

DF spectra of the chair-equatorial conformer (Figure 7.3) were obtained by pumping three vibronic bands: B, E, G. Unlike the chair-axial conformer, the DF spectrum pumping the LIF origin band of the chair-equatorial does not show strong transition and also signal to noise ratio was very bad. In the DF spectrum pumping Band B, the first peak other than the zero-redshift one is at $175 \mathrm{~cm}^{-1}$. This peak is hence assigned to the $\tilde{B} \rightarrow \tilde{A}$ origin band, corresponding to an $\tilde{A}-\tilde{X}$ separation of $175(8) \mathrm{cm}^{-1}$ for the chair-equatorial conformer. 
maybe assigned to the $\tilde{B} \rightarrow \tilde{A}$ origin band. Such an assignment is supported by other experimental evidence (vide infra).

DF spectrum pumping the LIF origin band of the chair-equatorial conformer (Band B) is significantly different from that of the chair-axial conformer. It is also the first member of a vibrational progression with separations of $\sim 703 \mathrm{~cm}^{-1}$ (see figure 7.4). It is therefore assigned to the transition to the first $\mathrm{C}-\mathrm{CH}_{3}$ stretch level of the $\tilde{X}$ state. The second member of the aforementioned progression is at $735 \mathrm{~cm}^{-1}, 910 \mathrm{~cm}^{-1}$ above the $\tilde{B} \rightarrow \tilde{A}$ origin band. Hence, this transition is assigned to the $\mathrm{C}_{-} \mathrm{CH}_{3}$ stretch level of the $\tilde{A}$ state. As a comparison density functional theory (DFT) calculations at the UCAM-B3LYP/6$311+\mathrm{G}(\mathrm{d})$ level of theory predicts fundamental frequencies of $708 \mathrm{~cm}^{-1}$ and $716 \mathrm{~cm}^{-1}$ for the $\tilde{X}$ state and $\tilde{A}$-state $\mathrm{C}_{-} \mathrm{CH}_{3}$ stretch modes, respectively.

The peak at $1441 \mathrm{~cm}^{-1}$ is assigned to the first overtone of the $\mathrm{C}_{-} \mathrm{CH}_{3}$ stretch of the $\tilde{X}$-state. The peak at $1584 \mathrm{~cm}^{-1}$ is assigned to the first overtone of the $\mathrm{C}-\mathrm{CH}_{3}$ stretch of the $\tilde{A}$-state. In both $\tilde{X}$ - and $\tilde{A}$-state transition, we did not observe the CO-stretch transition. Quantum calculation predicted the CO stretch frequencies $926 \mathrm{~cm}^{-1}$ and $918 \mathrm{~cm}^{-1}$ for $\tilde{X}$ and $\tilde{A}$-state assigned to the transition to the $\tilde{A}$-state CO-stretch level. However, in the $\mathrm{DF}$ spectrum, we did not observe any peak that can be assigned CO-stretch level. The strong peak at $1718 \mathrm{~cm}^{-1}$ can be assigned to the combination of $\mathrm{CO}$ stretch and $\mathrm{C}-\mathrm{CH}_{3}$ mode.

The strong peaks in the higher-frequency region contain multiple, partially resolved transitions. Transition frequencies were determined in multi-peak fitting to Gaussian shapes. Transition frequencies are summarized in Table 7.3. Three vibrational progressions 
are determined: $v_{\mathrm{C}-\mathrm{CH} 3}(\tilde{X}), v_{\mathrm{C}-\mathrm{CH} 3}(\tilde{A})$, and $(\mathrm{n}-2) v_{\mathrm{C}-\mathrm{CH} 3}(\tilde{X})+v_{\mathrm{CO}}(\tilde{X})$ with $\mathrm{n}=3-10$ (Figure 7.4). The constant intervals between the progression members suggest the absence of any detectable anharmonicity or Fermi resonance. Although transitions to the fundamental levels of the $\tilde{X}$ - and $\tilde{A}$-state $\mathrm{CO}$ stretch modes were not observed, their vibrational frequencies were determined by vibrational combination differences to be 703 $\mathrm{cm}^{-1}$ and $1015 \mathrm{~cm}^{-1}$, respectively.

In the present work of 1-MCHO, sharp $\mathrm{C}_{-} \mathrm{CH}_{3}$ stretch transitions were observed for $\tilde{X}$ - and $\tilde{A}$-state. In addition, the intensities of the DF peaks oscillate when the vibrational quantum numbers increase. The explanation of these observations based on computational results and theoretical analysis is given in Section 7.4. There is a noticeable difference when the origin (band B) band in the LIF spectrum was pumped, only four strong and resolved peaks $\left(0 \mathrm{~cm}^{-1}, 1718 \mathrm{~cm}^{-1}, 3230 \mathrm{~cm}^{-1}\right.$, and $\left.4715 \mathrm{~cm}^{-1}\right)$ were observed in the DF spectrum. These peaks are of equal spacing and are assigned to transitions to the vibrational ground level, the $v_{\mathrm{C}-\mathrm{CH} 3}+v_{\mathrm{CO}}$ level, the $3 v_{\mathrm{C}-\mathrm{CH} 3}+v_{\mathrm{CO}}$ level, and the $5 v_{\mathrm{C}-\mathrm{CH} 3}+v_{\mathrm{CO}}$ of the $\tilde{X}_{\text {state }}$

\subsection{Discussion}

The $\tilde{A}-\tilde{X}$ separation has been experimentally determined to $238 \mathrm{~cm}^{-1}$ for the chair-axial conformer and $175 \mathrm{~cm}^{-1}$ for the chair-equatorial conformer. These values shall be compared to DFT-calculated values of $286 \mathrm{~cm}^{-1}$ for the chair-axial conformer and $209 \mathrm{~cm}^{-1}$ for the chair-equatorial conformer (with zero-point-energy corrections). This is consistent 
with the previous conclusion that DFT calculations provide semi-quantitative prediction of the $\tilde{A}-\tilde{X}$ separation of alkoxy radicals.

The vibrational structure of the DF spectra of 1-MCHO is significantly different from those of the other three positional isomers. For 2-, 3-, and 4-MCHOs, DF spectra of emission from the vibrational ground level of the $\tilde{B}$ state are dominated by the CO-stretch progression. On the contrary, DF spectra, by pumping the LIF origin band of the chairaxial conformer of 1-MCHO, has strong progressions that involve both $\mathrm{CO}$ stretch and $\mathrm{C}$ $\mathrm{CH}_{3}$ stretch modes. Such difference is due to different positions of the methyl group on the six-membered ring and the steric interaction between the $\mathrm{CO}$ bond and the methyl group of 1-MCHO. For all MCHO isomers, the $\tilde{B} \leftarrow \tilde{X}$ excitation corresponds to the promotion of one electron in the $\mathrm{CO}$ bonding orbital to the half-filled orbital on the oxygen atom. It weakens the $\mathrm{CO}$ bond and hence increases the bond length. Such increment has been predicted by quantum chemical calculations to be $\sim 0.2 \AA$. Unlike the other three isomers, for 1-MCHO, the oxygen atom and the methyl group are connected to the same carbon atom. The weakening of the $\mathrm{CO}$ bond, therefore, allows the strengthening of the $\mathrm{C}-\mathrm{CH}_{3}$ bond due to less steric repulsion. The $\mathrm{C}-\mathrm{CH}_{3}$ bond length of the $\tilde{B}$-state is $35 \mathrm{~m} \AA$ shorter than that of the $\tilde{A} / \tilde{X}$ state according to quantum chemical calculations. Now that both $\mathrm{CO}$ and $\mathrm{C}-\mathrm{CH}_{3}$ bond lengths change significantly upon excitation, one expects to observe both $\mathrm{CO}$ stretch and $\mathrm{C}-\mathrm{CH}_{3}$ stretch transitions in the DF spectra of 1-MCHO.

The intensity pattern of the progressions of $\mathrm{CO}$ stretch and $\mathrm{C}-\mathrm{CH}_{3}$ stretch modes, however, does not follow that of a normal Franck-Condon progression. (See Figure 7.6 and 7.7) Figure 7.2 illustrates the DF spectrum of the chair-axial conformer in the region up to 
$6500 \mathrm{~cm}^{-1}$ pumping the LIF origin band. The intensities of the DF peaks demonstrate an oscillating pattern with intensity peaks at $v_{\mathrm{C}-\mathrm{CH} 3}(\tilde{X})$ and $\mathrm{n}_{\mathrm{C}-\mathrm{CH} 3}(\tilde{A})$ with $\mathrm{n}=1,2,4,5$. Such alternation of vibronic band intensity cannot be explained by vibronic interactions such as the pseudo-Jahn-Teller interaction between the $\tilde{A}$ - and $\tilde{X}$-states or coupling between vibration levels due to Fermi resonance and Coriolis interaction because the frequencies of vibrational levels are unperturbed and progressions with constant spacings have been observed. The most plausible explanation to the oscillating intensity pattern of DF peaks is, therefore, Duschinsky rotation, which affects the intensities of the vibronic bands but not their frequencies. Indeed, in the original paper on the effect later named after him, Duschinsky predicted that two mixed modes would have four combination bands with large transition intensities. ${ }^{122} \mathrm{~A}$ simulation was attempted but predicted strong $\mathrm{CO}$ stretch mode. The $\mathrm{CO}$ stretch mode and the $\mathrm{C}-\mathrm{CH}_{3}$ modes are mixed, which hinders quantitative analysis.

The DF spectra of 1-MCHO pumping the LIF origin band of both chair-axial and chair-equatorial are dominated by emission to the $\tilde{X}$-and $\tilde{A}$-states. The explanation of this observation is based on the vibronic interaction between the $\tilde{A}$ and $\tilde{X}$-state and was outlined in our previous work on the other three isomers. ${ }^{93}$ In brief, for all four isomers, the ground electronic $(\tilde{X})$ state has A" symmetry, while the first excited $(\tilde{A})$ state is an $\mathrm{A}^{\prime}$ state. The transition dipole moment (TDM) of the $\tilde{B} \mathrm{~A}^{\prime}-\tilde{A} \mathrm{~A}^{\prime}$ transition, lying within the $C_{s}$ plane, is dominant by the Herzberg-Teller (HT)-induced TDM due to the large difference between the permanent dipole moments (PDMs) of the two states. For the TDM of the $\tilde{B} \mathrm{~A}^{\prime}-\tilde{X} \mathrm{~A}^{\prime \prime}$ transition, which is perpendicular to the $C_{s}$ plane, its magnitude is expected to be close to 
the original (non-HT) TDM component. TDM of the $\tilde{B} \mathrm{~A}^{\prime}-\tilde{X} \mathrm{~A}^{\prime \prime}$ is therefore significantly weaker than the $\tilde{B} \mathrm{~A}^{\prime}-\tilde{A} \mathrm{~A}^{\prime}$ transition.

In the DF spectrum of the equatorial conformer pumping the LIF origin band, follow the similar pattern of axial-conformer. Also, DFT calculations, which have correctly predicted the energy ordering of other alkoxy radicals. Another significant difference between the DF spectra of $1-\mathrm{MCHO}$ and other MCHO isomers is that the former has narrow linewidth and partially resolved vibrational structure well into the high energy region, whereas for the latter, the members with large vibrational quantum numbers in the CO progressions are structureless.

DF spectra of the chair-equatorial conformer are lack of the strong progressions observed in that of the chair-axial conformer. This might be partly attributed to the low signal-to-noise ratio. Most probably, however, it is due to the more complicated PES and hence the vibronic structure of the $\tilde{X} / \tilde{A}$-state PES, a topic that is worth future investigation. Furthermore, emission from the CO-stretch levels of the $\tilde{B}$ state of both conformers shows only transitions to the $\mathrm{CO}$ stretch and $\mathrm{C}-\mathrm{CH}_{3}$ stretch combination levels of the $\tilde{X}$ state. No CO stretch transitions to the $\tilde{A}$-state were observed.

\subsection{Conclusions}

The $\tilde{A}-\tilde{X}$ separation of the chair-axial and chair-equatorial conformers of the 1 -MCHO radical has been experimentally determined to be $238 \mathrm{~cm}^{-1}$ and $175 \mathrm{~cm}^{-1}$, respectively. When the LIF origin band of the chair-axial conformer of 1-MCHO is pumped, its DF 
spectrum is dominated by three progressions of $\mathrm{C}-\mathrm{O}$ stretch and $\mathrm{C}-\mathrm{CH}_{3}$ stretch modes. The intensity pattern of the progressions can be explained by Duschinsky mixing between these two modes. 
Table 7.1. Numbers of conformers and their calculated relative energies, energy separation between $\tilde{A}$-and $\tilde{X}$-states $\left(\Delta E^{\tilde{A}-\tilde{X}}\right)$ and $\tilde{X}$ - and $\tilde{A}$-state $\mathrm{CO}$-stretch and $\mathrm{C}_{-} \mathrm{CH}_{3}$-stretch frequencies of 1-MCHO conformers. $\Delta E^{\tilde{A}-\tilde{X}}$ includes zero-point-energy corrections.

\begin{tabular}{ccccccc}
\hline conformer & $\begin{array}{c}\text { relative } \\
\text { energy }\left(\mathrm{cm}^{-1}\right)\end{array}$ & $\Delta E^{\tilde{A}-\tilde{X}}$ & $v_{\mathrm{CO}}^{\tilde{\mathrm{X}}_{\mathrm{CO}}\left(\mathrm{cm}^{-1}\right)}$ & $v_{\mathrm{CO}}^{\tilde{\tilde{A}}}\left(\mathrm{~cm}^{-1}\right)$ & $v_{\mathrm{C}-\mathrm{CH} 3}^{\tilde{x}}\left(\mathrm{~cm}^{-1}\right)$ & $v_{\mathrm{C}-\mathrm{CH} 3}\left(\mathrm{~cm}^{-1}\right)$ \\
\hline axial & 0 & 286 & 925 & 919 & 553 & 569 \\
equatorial & 420 & 209 & 926 & 918 & 708 & 716 \\
\hline
\end{tabular}


Table 7.2. Redshifts (r.s.) from the pumped LIF lines and relative wavenumbers (rel.) observed in the DF spectra of the chair-axial conformer of 1-MCHO $\left(\mathrm{cm}^{-1}\right)$.

\begin{tabular}{|c|c|c|c|c|}
\hline r.s. & cald. & rel. $^{a}$ & cald. & assignment \\
\hline 0 & & & & $0_{0}^{0}(\tilde{\mathrm{B}} \rightarrow \tilde{\mathrm{X}})$ \\
\hline 238 & & & & $0_{0}^{0}(\tilde{\mathrm{B}} \rightarrow \tilde{\mathrm{A}})$ \\
\hline 486 & 553 & & & $v_{\mathrm{C}-\mathrm{CH} 3}(\tilde{\mathrm{X}})$ \\
\hline 755 & & 517 & 569 & $v_{\mathrm{C}-\mathrm{CH} 3}(\tilde{\mathrm{A}})$ \\
\hline 996 & & & & $2 v_{\mathrm{C}-\mathrm{CH} 3}(\tilde{\mathrm{X}})$ \\
\hline 1267 & & 1029 & & $2 v_{\mathrm{C}-\mathrm{CH} 3}(\tilde{\mathrm{A}})$ \\
\hline 1491 & & & & $3 v_{\mathrm{C}-\mathrm{CH} 3}(\tilde{\mathrm{X}})$ \\
\hline 1578 & & & & $v_{\mathrm{C}-\mathrm{CH} 3}(\tilde{\mathrm{X}})+v_{\mathrm{CO}}(\tilde{\mathrm{X}})$ \\
\hline 1800 & & 1562 & & $3 v_{\mathrm{C}-\mathrm{CH} 3}(\tilde{\mathrm{A}})$ \\
\hline 2060 & & & & $4 v_{\mathrm{C}-\mathrm{CH} 3}(\tilde{\mathrm{X}})$ \\
\hline 2315 & & 2077 & & $4 v_{\mathrm{C}-\mathrm{CH} 3}(\tilde{\mathrm{A}})$ \\
\hline 2618 & & & & $5 v_{\mathrm{C}-\mathrm{CH} 3}(\tilde{\mathrm{X}})$ \\
\hline 2849 & & 2611 & & $5 v_{\mathrm{C}-\mathrm{CH} 3}(\tilde{\mathrm{A}})$ \\
\hline 3055 & & & & $6 v_{\mathrm{C}-\mathrm{CH} 3}(\tilde{\mathrm{X}})$ \\
\hline 3126 & & & & $4 v_{\mathrm{C}-\mathrm{CH} 3}(\tilde{\mathrm{X}})+v_{\mathrm{CO}}(\tilde{\mathrm{X}})$ \\
\hline 3341 & & 3103 & & $6 v_{\mathrm{C}-\mathrm{CH} 3}(\tilde{\mathrm{A}})$ \\
\hline 3562 & & & & $7 v_{\mathrm{C}-\mathrm{CH} 3}(\tilde{\mathrm{X}})$ \\
\hline 3846 & & 3608 & & $7 v_{\mathrm{C}-\mathrm{CH} 3}(\tilde{\mathrm{A}})$ \\
\hline 4072 & & & & $8 v_{\mathrm{C}-\mathrm{CH} 3}(\tilde{\mathrm{X}})$ \\
\hline 4374 & & 4136 & & $8 v_{\mathrm{C}-\mathrm{CH} 3}(\tilde{\mathrm{A}})$ \\
\hline 4543 & & & & $9 v_{\mathrm{C}-\mathrm{CH} 3}(\tilde{\mathrm{X}})$ \\
\hline 4628 & & & & $7 v_{\mathrm{C}-\mathrm{CH} 3}(\tilde{\mathrm{X}})+v_{\mathrm{CO}}(\tilde{\mathrm{X}})$ \\
\hline 4855 & & 4617 & & $9 v_{\mathrm{C}-\mathrm{CH} 3}(\tilde{\mathrm{A}})$ \\
\hline 5057 & & & & $10 v_{\mathrm{C}-\mathrm{CH} 3}(\tilde{\mathrm{X}})$ \\
\hline 5375 & & 5137 & & $10 v_{\mathrm{C}-\mathrm{CH} 3}(\tilde{\mathrm{A}})$ \\
\hline 5565 & & & & $11 v_{\mathrm{C}-\mathrm{CH} 3}(\tilde{\mathrm{X}})$ \\
\hline 5876 & & 5638 & & $11 v_{\mathrm{C}-\mathrm{CH} 3}(\tilde{\mathrm{A}})$ \\
\hline 6062 & & & & $12 v_{\mathrm{C}-\mathrm{CH} 3}(\tilde{\mathrm{X}})$ \\
\hline 6115 & & & & $10 v_{\mathrm{C}-\mathrm{CH} 3}(\tilde{\mathrm{X}})+v_{\mathrm{CO}}(\tilde{\mathrm{X}})$ \\
\hline 6371 & & 6133 & & $12 v_{\mathrm{C}-\mathrm{CH} 3}(\tilde{\mathrm{A}})$ \\
\hline
\end{tabular}

${ }^{\text {a }}$ Redshift relative to the $\tilde{A}-\tilde{X}$ separation $\left(238 \mathrm{~cm}^{-1}\right)$ 
Table 7.3. Redshifts (r.s.) from the pumped LIF lines and relative wavenumbers (rel.) observed in the DF spectra of the chair-equatorial conformer of 1-MCHO $\left(\mathrm{cm}^{-1}\right)$.

\begin{tabular}{|c|c|c|c|c|}
\hline r.s. & cald. & rel. $^{a}$ & cald. & assignment \\
\hline 0 & & & & $0_{0}^{0}(\tilde{\mathrm{B}} \rightarrow \tilde{\mathrm{X}})$ \\
\hline 175 & & & & $0_{0}^{0}(\tilde{\mathrm{B}} \rightarrow \tilde{\mathrm{A}})$ \\
\hline 703 & 708 & & & $v_{\mathrm{C}-\mathrm{CH} 3}(\tilde{\mathrm{X}})$ \\
\hline 910 & & 735 & 716 & $v_{\mathrm{C}-\mathrm{CH} 3}(\tilde{\mathrm{A}})$ \\
\hline 1441 & & & & $2 v_{\mathrm{C}-\mathrm{CH} 3}(\tilde{\mathrm{X}})$ \\
\hline 1584 & & 1409 & & $2 v_{\mathrm{C}-\mathrm{CH} 3}(\tilde{\mathrm{A}})$ \\
\hline 1718 & & & & $v_{\mathrm{C}-\mathrm{CH} 3}(\tilde{\mathrm{X}})+v_{\mathrm{CO}}(\tilde{\mathrm{X}})$ \\
\hline 2219 & & & & $3 v_{\mathrm{C}-\mathrm{CH} 3}(\tilde{\mathrm{X}})$ \\
\hline 2409 & & 2234 & & $3 v_{\mathrm{C}-\mathrm{CH} 3}(\tilde{\mathrm{A}})$ \\
\hline 2930 & & & & $4 v_{\mathrm{C}-\mathrm{CH} 3}(\tilde{\mathrm{X}})$ \\
\hline 3126 & & 2951 & & $4 v_{\text {C-CH3 }}(\tilde{\mathrm{A}})$ \\
\hline 3230 & & & & $3 v_{\mathrm{C}-\mathrm{CH} 3}(\tilde{\mathrm{X}})+v_{\mathrm{CO}}(\tilde{\mathrm{X}})$ \\
\hline 3644 & & & & $5 v_{\mathrm{C}-\mathrm{CH} 3}(\tilde{\mathrm{X}})$ \\
\hline 3771 & & 3596 & & $5 v_{\mathrm{C}-\mathrm{CH} 3}(\tilde{\mathrm{A}})$ \\
\hline 4290 & & & & $6 v_{\mathrm{C}-\mathrm{CH} 3}(\tilde{\mathrm{X}})$ \\
\hline 4487 & & 4312 & & $6 v_{\mathrm{C}-\mathrm{CH} 3}(\tilde{\mathrm{A}})$ \\
\hline 4775 & & & & $5 v_{\mathrm{C}-\mathrm{CH} 3}(\tilde{\mathrm{X}})+v_{\mathrm{CO}}(\tilde{\mathrm{X}})$ \\
\hline 4902 & & & & $7 v_{\mathrm{C}-\mathrm{CH} 3}(\tilde{\mathrm{X}})$ \\
\hline 5233 & & 5058 & & $7 v_{\mathrm{C}-\mathrm{CH} 3}(\tilde{\mathrm{A}})$ \\
\hline 5603 & & & & $8 v_{\mathrm{C}-\mathrm{CH} 3}(\tilde{\mathrm{X}})$ \\
\hline 6220 & & & & $7 v_{\mathrm{C}-\mathrm{CH} 3}(\tilde{\mathrm{X}})+v_{\mathrm{CO}}(\tilde{\mathrm{X}})$ \\
\hline
\end{tabular}

${ }^{\text {a }}$ Redshift relative to the $\tilde{A}-\tilde{X}$ separation $\left(175 \mathrm{~cm}^{-1}\right)$ 


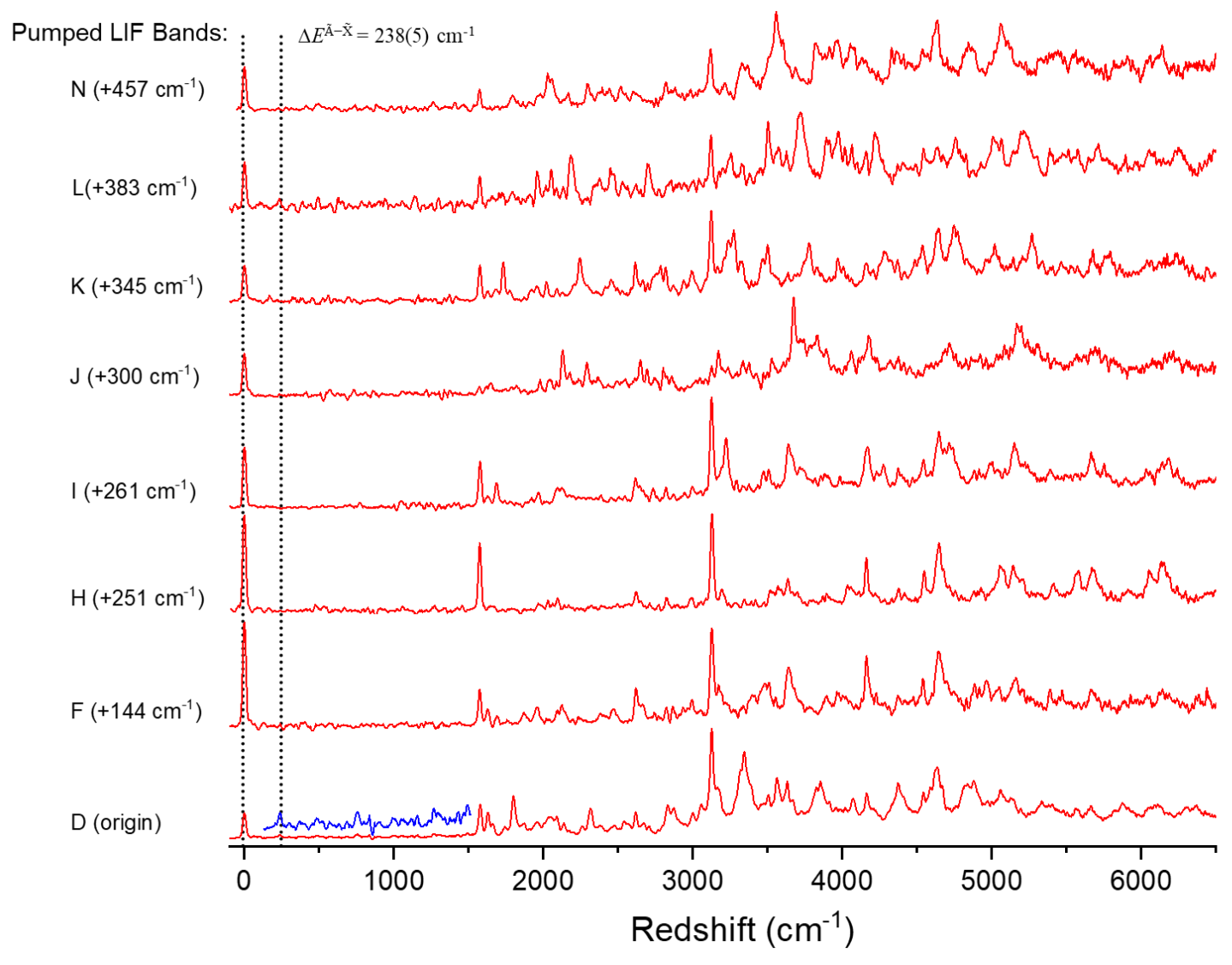

Figure 7.1. DF spectra of the chair-axial conformer of 1-MCHO. Numbers in parentheses after the LIF bands are relative wavenumbers with respect to the origin band (Band D) at $27614 \mathrm{~cm}^{-1}$. 


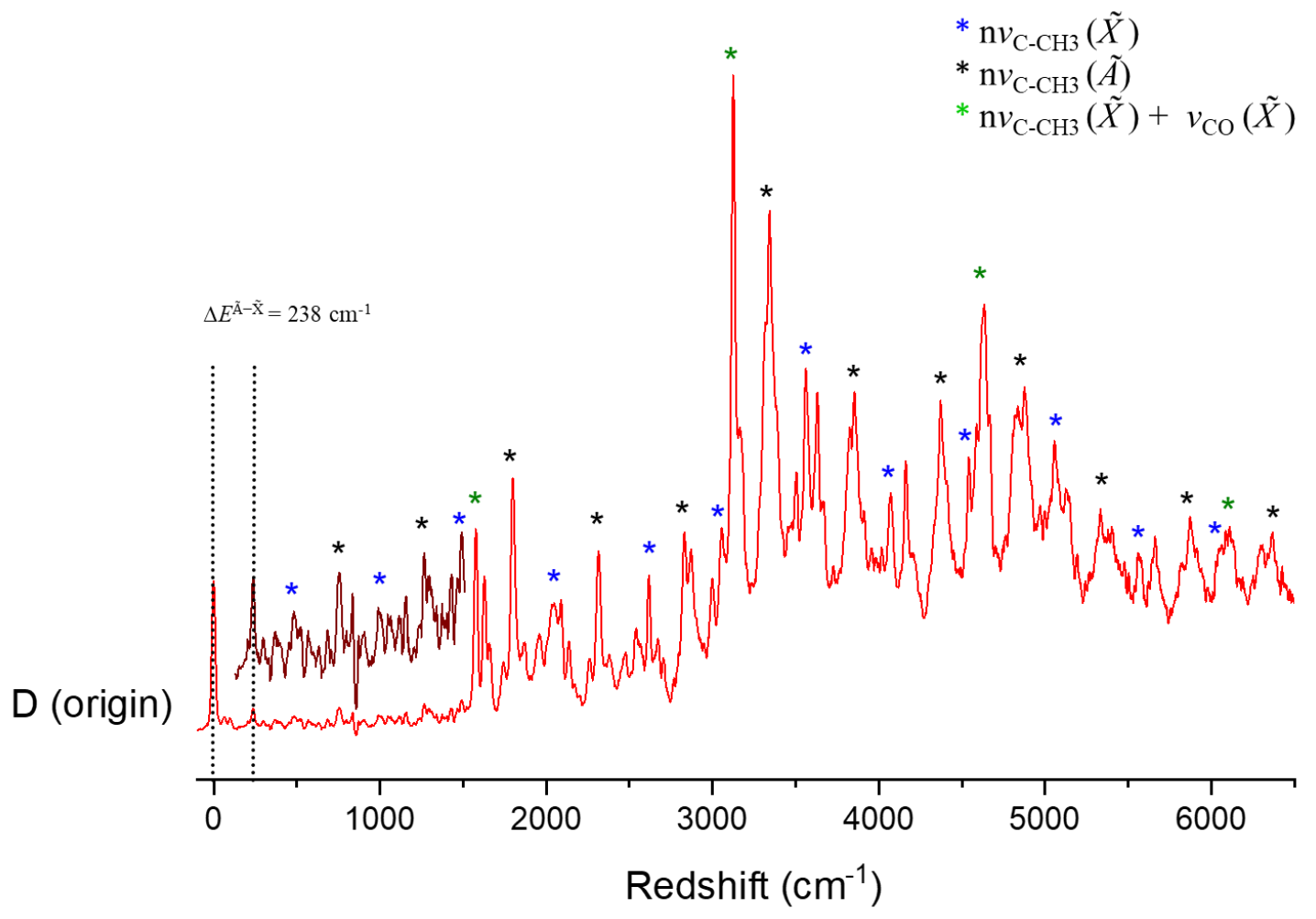

Figure 7.2. DF spectrum of band D $\left(27614 \mathrm{~cm}^{-1}\right)$ in the LIF of the chair-axial conformer of 1-MCHO. The asterisks are the assignments of the DF spectrum, $v_{\mathrm{C}-\mathrm{CH} 3}(\tilde{X}), v_{\mathrm{C}-\mathrm{CH} 3}$ $(\tilde{A})$, and $v_{\mathrm{C}-\mathrm{CH} 3}(\tilde{X})+v_{\mathrm{CO}}(\tilde{X})$, respectively. 


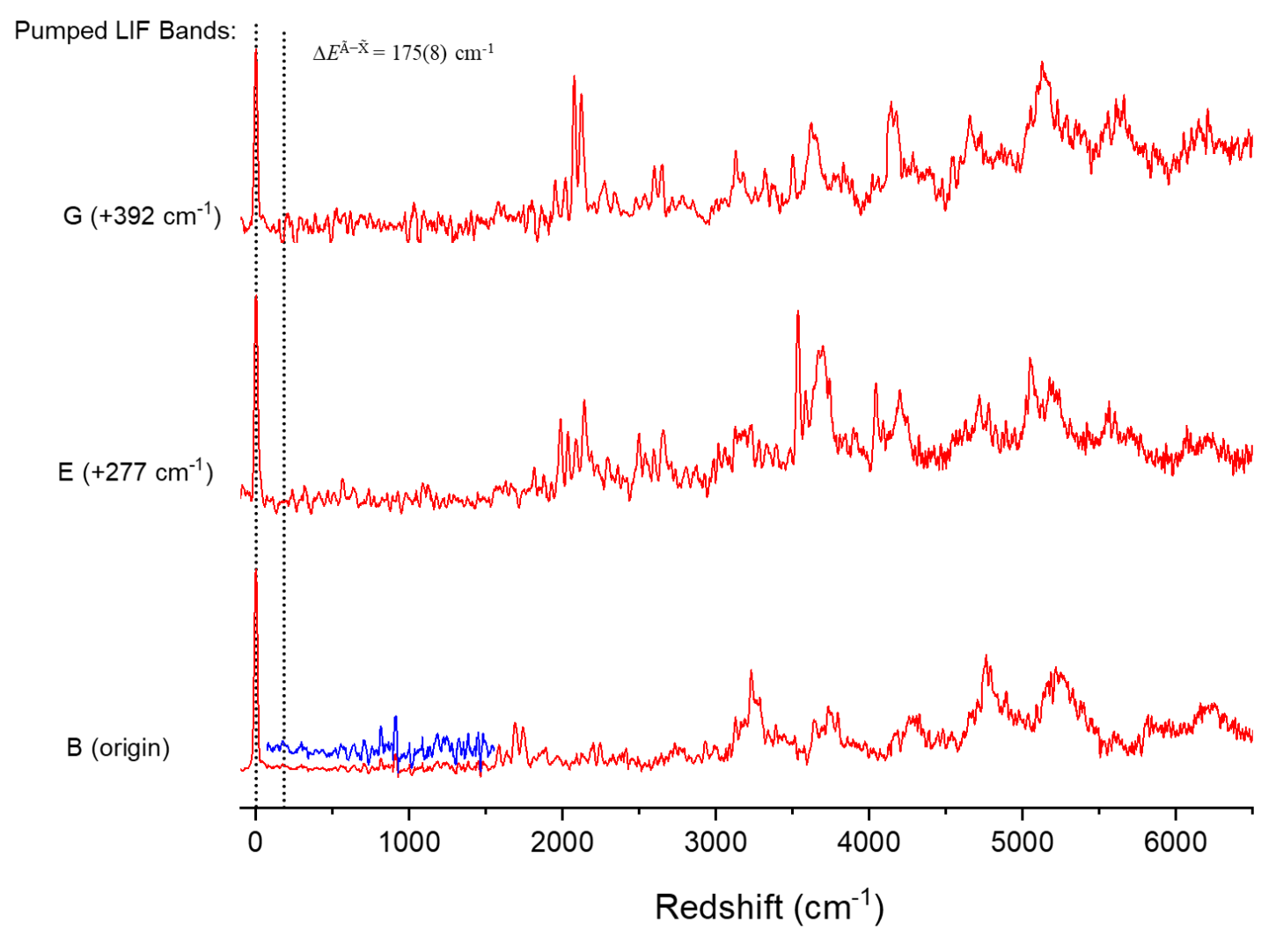

Figure 7.3. DF spectra of the chair-equatorial conformer of 1-MCHO. Numbers in parentheses after the LIF bands are relative wavenumbers with respect to the origin band (Band B) at $27455 \mathrm{~cm}^{-1}$. 


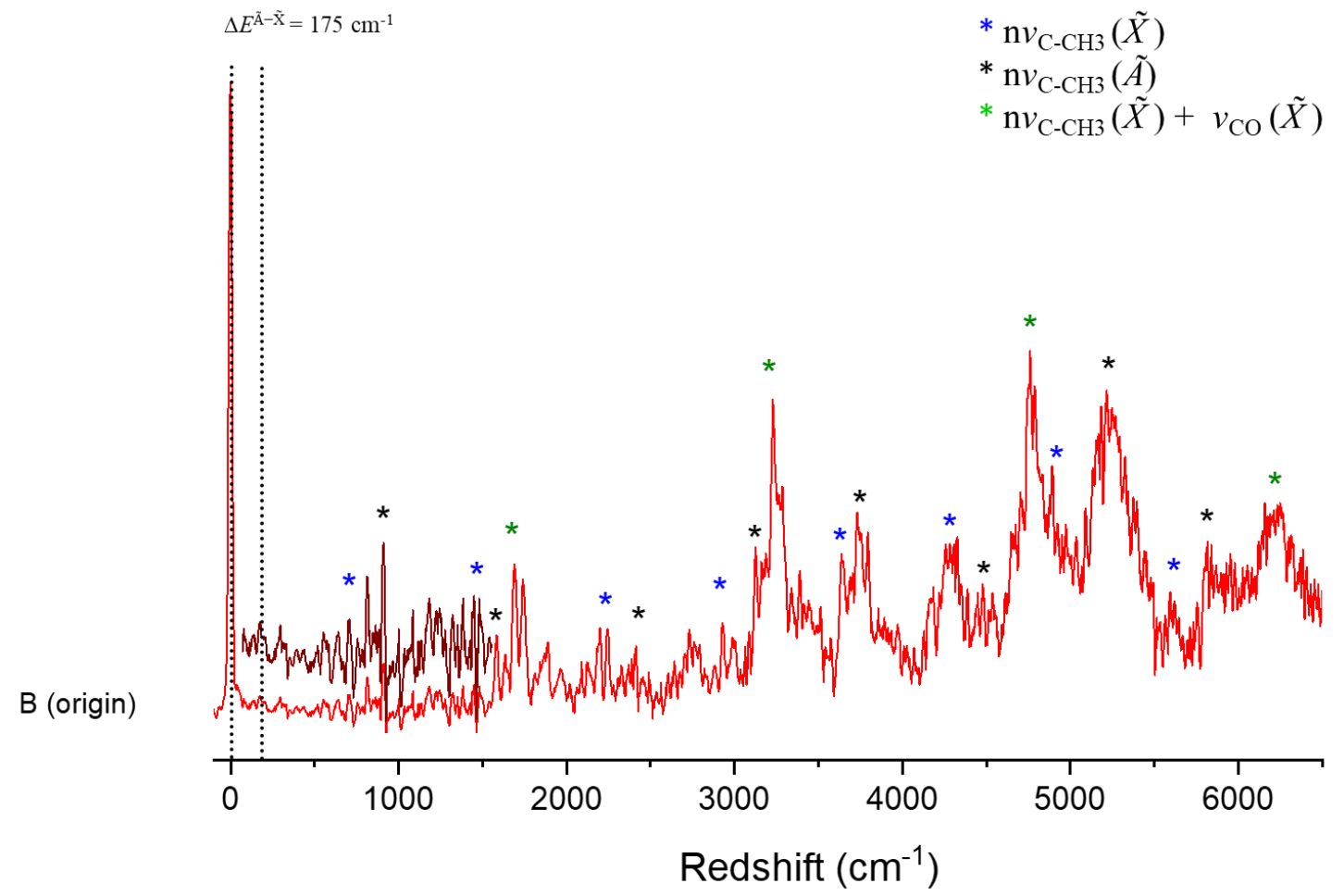

Figure 7.4. DF spectrum of band B $\left(27455 \mathrm{~cm}^{-1}\right)$ in the LIF spectrum of the chairequatorial conformer of $1-\mathrm{MCHO}$. The asterisks are the assignments of the DF spectrum, $v_{\mathrm{C}-\mathrm{CH} 3}(\tilde{X}), v_{\mathrm{C}-\mathrm{CH} 3}(\tilde{A})$, and $v_{\mathrm{C}-\mathrm{CH} 3}(\tilde{X})+v_{\mathrm{CO}}(\tilde{X})$, respectively. 


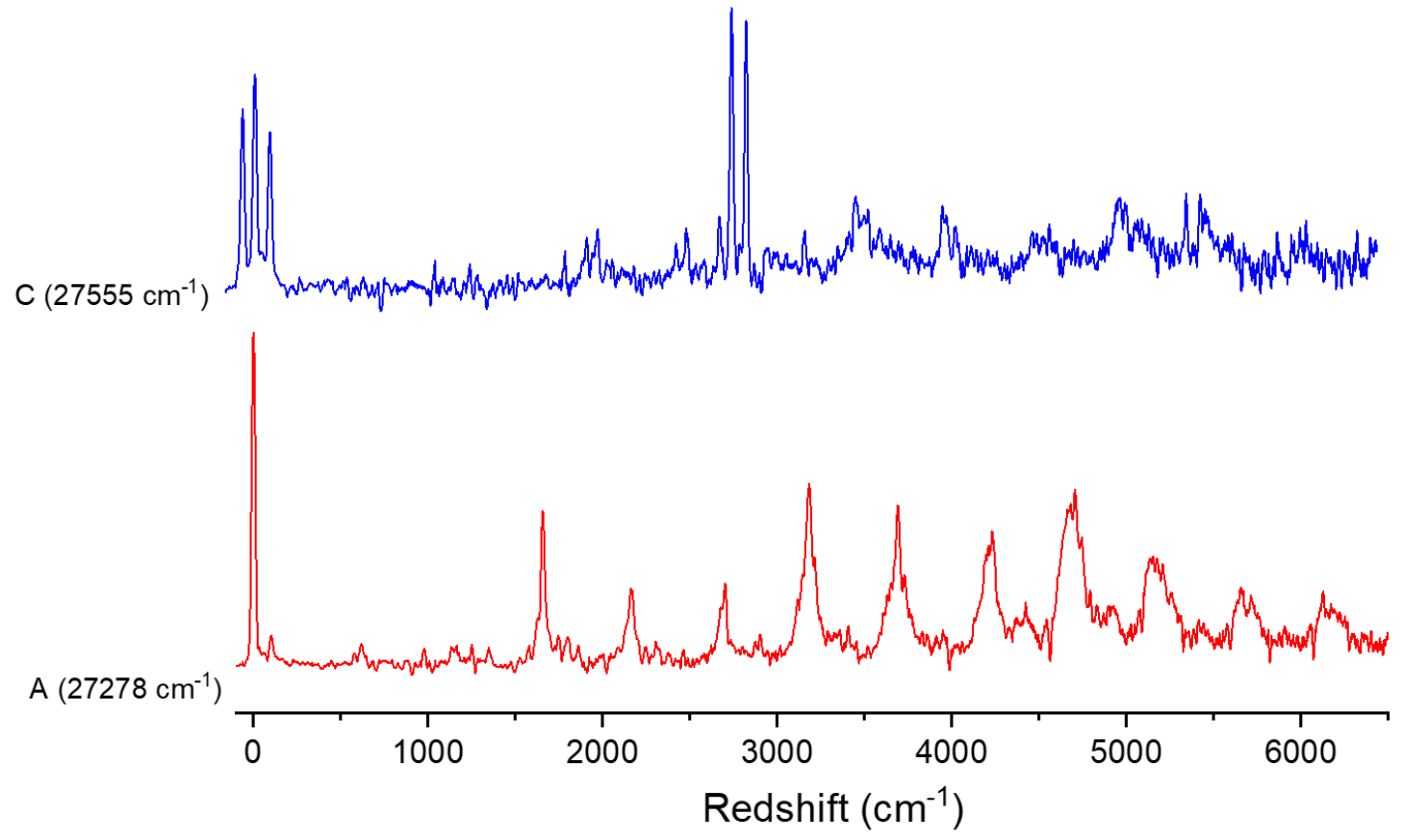

Figure 7.5. DF spectra of unknown LIF bands of 1-MCHO. Numbers in parentheses are the LIF frequencies. 


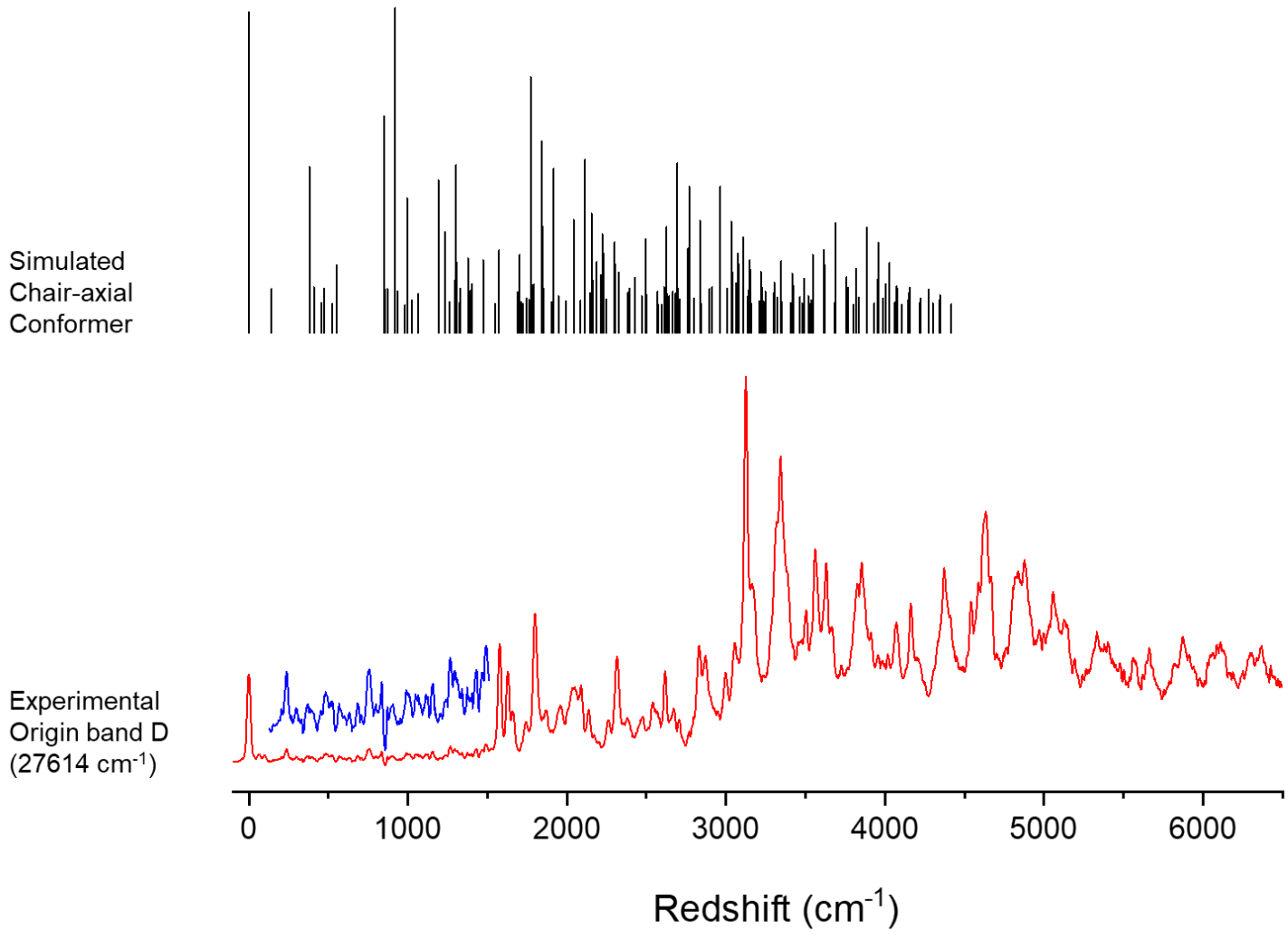

Figure 7.6. Comparison between the experimental (bottom red trace) and simulated (top black trace) DF spectra of the chair-axial conformer of the 1-MCHO radical. The experimental DF spectra were obtained by pumping the origin band in the LIF spectrum (Band D). 


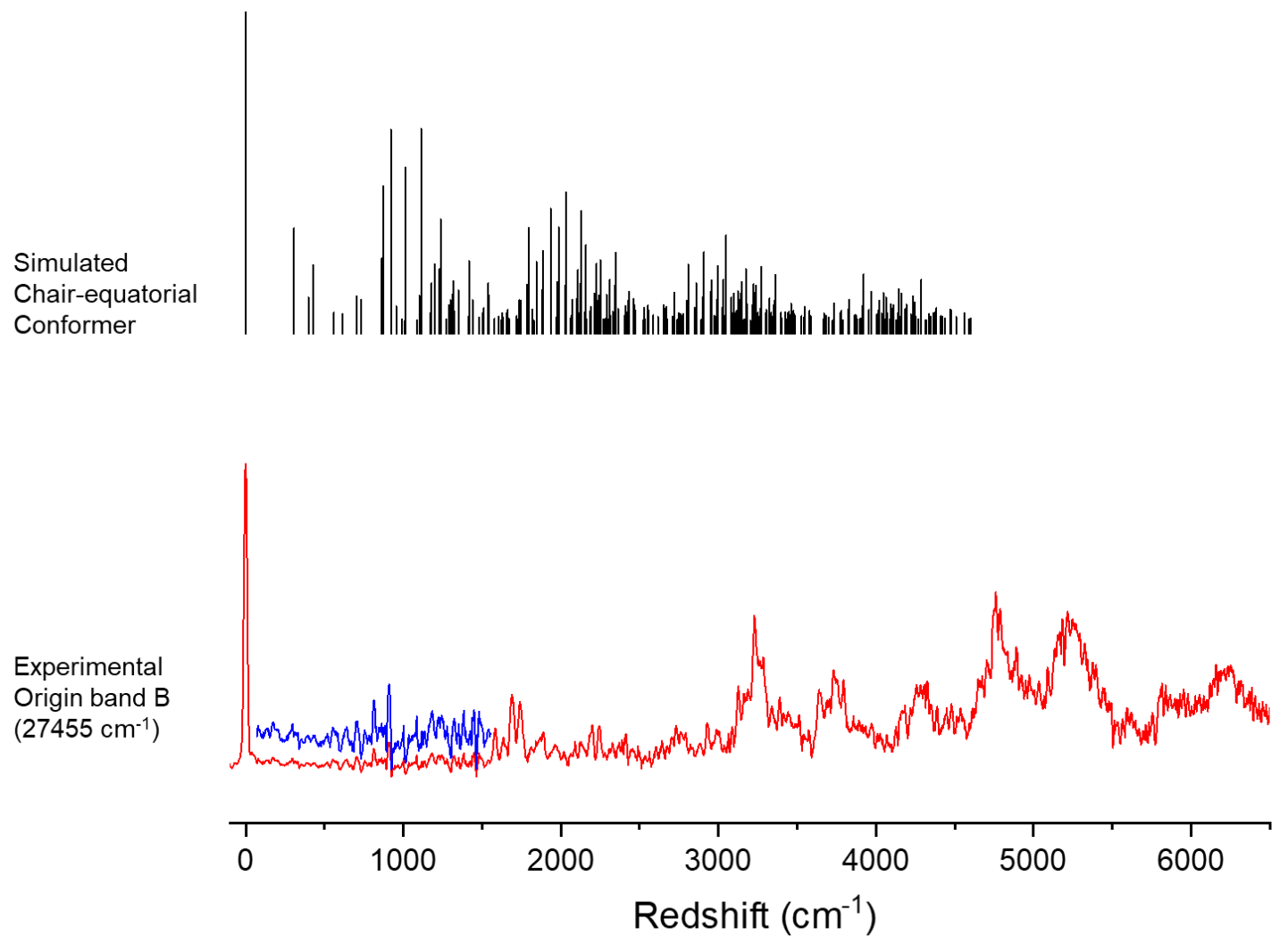

Figure 7.7. Comparison between the experimental (bottom red trace) and simulated (top black trace) DF spectra of the chair-equatorial conformer of the 1-MCHO radical. The experimental DF spectra were obtained by pumping the origin band in the LIF spectrum (Band B). 


\section{CHAPTER VIII}

\section{ROOM-TEMPERATURE CAVITY RING-DOWN SPECTROSCOPY OF METHYLALLYL PEROXY RADICALS}

\subsection{Introduction}

Peroxy radicals are key reaction intermediates formed during the oxidation of hydrocarbons in the atmosphere, ${ }^{2,39}$ and low-temperature combustion. ${ }^{40,133}$ Although spectroscopy and kinetics of saturated straight-chain peroxy radicals have been extensively investigated, and fewer studies have been done on unsaturated peroxy radicals. ${ }^{72,152-154}$ In particular, many intermediates in the oxidation of conjugated olefins contain the allyl peroxy radical functionality. ${ }^{68,71}$ This functionality is also present in four of the six possible isomers of peroxy radicals formed in $\mathrm{OH}$-initiated oxidation of 2-methyl-1,3-butadiene, viz. isoprene,$^{71}$ the most abundant non-methane biogenic hydrocarbon. ${ }^{155,156}$ Although direct spectroscopic or kinetic measurement of the peroxy radicals in isoprene oxidation has not been reported, their base unit, the allyl peroxy radical was observed via roomtemperature cavity ring-down (CRD) spectroscopy. ${ }^{72}$ Using ab initio calculated molecular constants, rotational contours of all five conformers of allyl peroxy were generated and 
used to simulate experimentally obtained CRD spectrum of the radical. Allyl peroxy is the smallest peroxy radical with the allyl functional group. Its alkyl substitution introduces more isomer and complicates the conformational landscape. Moreover, chemical reactions that produce substituted allyl peroxies involve intermediates that possess multiple resonance structures, which leads to complex reaction kinetics. Here we report roomtemperature CRD spectra of the methylallyl peroxy (MAOO') radical. MAOO has three positional isomers (1-, 2-, and 3-MAOO'). Each isomer has multiple rotational conformers or rotamers. Limited by spectral linewidth, transitions of individual conformers were not resolved in the present work. Nevertheless, spectral simulation aided by quantum chemical calculations allows identification of molecular carriers of recorded CRD spectra, which is essential to future kinetic studies. Furthermore, simulating the experimental spectra helps understand chemical reactions that produce the MAOO radicals and the role of resonancestabilized free radical intermediates in the process.

\subsection{Calculations}

The PES of each MAOO isomer is complicated by the existence of multiple conformations. In this section, we first provide an overview of the PES landscapes and the

conformational search. Geometry optimization and energy calculation of both $\tilde{X}$ - and $\tilde{A}$ state molecules will follow. They provide molecular constants and other quantities that are necessary for spectral simulation, including relative populations of different conformers of each isomer, frequencies, and oscillator strengths of their $\tilde{A} \leftarrow \tilde{X}$ electronic transitions, vibrational frequencies, and Franck-Condon factors (FCFs) of the vibronic transitions. 


\subsubsection{PES Survey and Conformational Search}

The conformational search of the MAOO radical has been done on the basis of the allyl peroxy $\left(\mathrm{CH}_{2}=\mathrm{CHCH}_{2} \mathrm{OO}\right)$ radical, ${ }^{72}$ which has two dihedral angles $\left(\Phi_{1}\right.$ : $\mathrm{OOC}_{3} \mathrm{C}_{2}$ and $\Phi_{2}$ : $\mathrm{OC}_{3} \mathrm{C}_{2}=\mathrm{C}_{1}$ ). Each dihedral angle can have three orientations in stable conformers: trans (T), counterclockwise gauche $(\mathrm{G})$, and clockwise gauche $\left(\mathrm{G}^{\prime}\right)$ for $\Phi_{1}$; cis $(\mathrm{C}), \mathrm{G}$, and $\mathrm{G}^{\prime}$ for $\Phi_{2}$. Among the total $3 \times 3=9$ stable conformers, there are four pairs of enantiomers, which belong to the $C_{1}$ point group. Therefore, the allyl peroxy radical has five optically distinguishable conformers. For MAOO, we label the carbon atoms the same way would the allyl peroxy radical. The carbon atom in the substituting methyl group is labeled " $\mathrm{C}_{\mathrm{m}}$." (See Figure 8.2)

1-MAOO, a straight-chain molecule, has three dihedral angles: $\Phi_{1}$ : $\mathrm{OOC}_{3} \mathrm{C}_{2}, \Phi_{2}$ : $\mathrm{OC}_{3} \mathrm{C}_{2}=\mathrm{C}_{1}$, and $\Phi_{3}: \mathrm{C}_{3} \mathrm{C}_{2}=\mathrm{C}_{1} \mathrm{C}_{\mathrm{m}}$. Its conformations are therefore named according to these dihedral angles. Like in the allyl peroxy radical, rotation about a non- $\mathrm{CH}$ single bond introduces three conformations. If the rotating bond is a single bond and both adjacent bonds are single bonds too, e.g., $\Phi_{1}$, the three possible conformations are $\mathrm{T}$ (trans- with $\Phi \sim 180^{\circ}$ ), $\mathrm{G}$ (gauche with $\Phi \sim 60^{\circ}$ counterclockwise), and $\mathrm{G}^{\prime}$ (gauche with $\Phi \sim 60^{\circ}$ clockwise). If the rotating bond is a single bond and one of the adjacent bonds is a double bond, e.g., $\Phi_{2}$, the three conformations are: C (cis- with $\Phi \sim 0^{\circ}$ ), G (gauche with $\Phi \sim 120^{\circ}$ counterclockwise), and $\mathrm{G}^{\prime}$ (gauche with $\Phi \sim 120^{\circ}$ clockwise). Rotation about a $\mathrm{C}=\mathrm{C}$ double bond, e.g., $\Phi_{3}$, introduces two conformations $\mathrm{C}$ (cis- with $\Phi \sim 0^{\circ}$ ) and $\mathrm{T}$ (trans- with $\left.\Phi \sim 180^{\circ}\right)$. In total, there are $3 \times 3 \times 2=18$ conformations. Two of these conformations, namely, TCC and TCT, belong to the $C_{\text {s }}$ point group. The rest of the 16 conformations, belonging to the $C_{1}$ point group, form eight pairs of enantiomers, which reduces the total 
number of optically distinguishable conformations to ten. Geometry optimization showed that GCC $\left(C_{l}\right)$ and one of the $C_{s}$ conformations, namely, TCC $\left(C_{s}\right)$, are actually transition structures. 1-MAOO, therefore, has eight optically distinguishable stable conformers.

2-MAOO can be derived from allyl peroxy by methyl substitution at the $\mathrm{C}_{2}$ position in the parent chain. Because there is only one hydrogen atom connected to $\mathrm{C}_{2}$ and internal rotation of the methyl group does not introduce new conformations, the number of optically distinguishable conformations of 2-MAOO is the same as the allyl peroxy radical, i.e., 5 . They are named according to the dihedral angles $\Phi_{1}$ : $\mathrm{OOC}_{3} \mathrm{C}_{2}$ and $\Phi_{2}$ : $\mathrm{OC}_{3} \mathrm{C}_{2}=\mathrm{C}_{1}$. Geometry optimization shows that all five conformations are stable structures.

Similarly, 3-MAOO can be viewed as methyl substitution of allyl peroxy, where the methyl group is attached to $\mathrm{C}_{3}$. In the allyl peroxy radical, there are two hydrogen atoms connected to $\mathrm{C}_{3}$. They are equivalent for the $\mathrm{TC}\left(C_{\mathrm{s}}\right)$ conformation but distinct for the rest $4 C_{1}$ ones. $3-\mathrm{MAOO}$, therefore, has $1 \times 1+4 \times 2=9$ optically distinguishable conformations. The conformations of 3-MAOO are named again by $\Phi_{1}$ : $\mathrm{OOC}_{3} \mathrm{C}_{2}$ and $\Phi_{2}$ : $\mathrm{OC}_{3} \mathrm{C}_{2} \mathrm{C}_{1}$. Since the dioxygen moiety and $\mathrm{C}_{\mathrm{m}}$ are at the same center, the third dihedral angle, $\Phi_{3}$ : $\mathrm{C}_{\mathrm{m}} \mathrm{C}_{3} \mathrm{C}_{2} \mathrm{C}_{1}$, is predetermined given a certain combination of $\Phi_{1}$ and $\Phi_{2}$. It hence does not introduce new conformations. All nine conformations are stable structures. Since all 3MAOO conformers belong to the $C_{1}$ point group, each of them has its enantiomer.

Table 8.1 summarizes the numbers of (optically distinguishable) conformations, transition structures, and stable conformers of all three isomers of MAOO. Newman's projections are illustrated in Figures 8.8-10. 


\subsubsection{Quantum Chemical Calculations}

Density functional theory (DFT) calculations at the B3LYP/6-31+G(d,p) level of theory were carried out on ground-state MAOO conformers using the Gaussian 09 software package. ${ }^{114}$ Relative energies of conformers with zero-point energy corrections are listed in Tables 8.2 and 8.3. Time-dependent (TD-)DFT calculations at the same level were employed for calculations of $\tilde{A}$-state molecules. Geometries and vibrational frequencies of both states were predicted in the calculations. In addition, vertical and adiabatic excitation

energies of the $\tilde{A} \leftarrow \tilde{X}$ transition were determined. The calculated geometric parameters of all conformers are summarized in Table 8.4.

\subsubsection{Spin Densities of the 1-MA Radial and PES Scan Along the Reaction Coordinate} of Oxygen Addition

The 1-MA radical has two resonance structures $\left(\mathrm{CH}_{3} \mathrm{CH}=\mathrm{CHCH}_{2} \cdot\right.$ and $\left.\mathrm{CH}_{3} \mathrm{CH} \cdot \mathrm{CH}=\mathrm{CH}_{2}\right)$. Their population ratio at equilibrium is determined by the nature and extent of electron delocalization. Atomic partition of the spin density at the B3LYP/6-31+G(d,p) level of theory ${ }^{157}$ finds that $52 \%$ of the spin is on the terminal $\mathrm{C}$ and $48 \%$ on the non-terminal one (Figure 8.3).

In order to compare reaction rate constants $k_{1-\mathrm{MAOO}}$ and $k_{3-\mathrm{MAOO}}$, the Complete Basis Set (CBS)-QB3 method was employed to scan the PESs along the reaction coordinate of oxygen addition. In these calculations, the distance between the $\mathrm{C}_{3}$ atom and the center of the $\mathrm{OO}$ moiety is varied from $1.5 \AA$ to $6.0 \AA$. Other coordinates are optimized at each step of the PES scan. Since the gauche conformer of the 1-MA radical is significantly lower in 
energy than its cis conformer, only the lowest-energy conformers of 1- and 3-MAOO with a gauche CCCC dihedral angle are included. The scans of PESs along the reaction coordinates are plotted in Figure 8.4. Oxygen addition on either the terminal or the nonterminal positions has a small barrier $(<3 \mathrm{kcal} / \mathrm{mol})$ because of the elimination of the allylic resonance. ${ }^{158}$ Both the heights of the barriers and the depth of the wells are similar for the production of 1 - and 3-MAOO's. Reaction rate constants $k_{1-\mathrm{MAOO}}$ and $k_{3-\mathrm{MAOO}}$, are therefore expected to be close.

Because the two free radical positions of 1-MA have similar spin densities and the reaction rates for oxygen addition on these two positions are expected to be similar, the branching ratio between production of 1- and 3-MAOO's using either 1-chloro-2-butene or 3-chloro-1-butene as a precursor (Reactions 5 and 6) is expected to be 1:1.

\subsection{Spectral Simulation and Assignment}

Calculated adiabatic $\tilde{A} \leftarrow \tilde{X}$ transition frequencies of MAOO conformers are used to simulate experimentally observed spectra. Calculation of transition intensities takes into consideration the following factors:

\subsubsection{Relative Populations of MAOO Isomers}

When 3-chloro-2-methyl-1-propene is used as the precursor, only one of the three isomers of MAOO, namely, 2-MAOO, can be produced. Its relative population is therefore set to unity. Both 1- and 3-MAOO isomers can be produced using either 1-chloro-2-butene or 3chloro-1-butene as a precursor. Based on the discussion above, populations of these two 
isomers are expected to be close. In the spectral simulation, weights of these two isomers are set to 52:48, the ratio of spin densities on the terminal and non-terminal positions of the 1-MA radical.

\subsubsection{Population Ratios of Different Conformers of Each Isomer}

PES scans, along with reaction coordinates that connect different rotamers of each isomer of MAOO, are illustrated in Figures 8.11-16. The energy barriers are relatively low $(<3$ $\mathrm{kcal} / \mathrm{mol}$ ). In the spectra simulation, we assumed that relative populations of all conformers of each isomer follow the Boltzmann distribution. The Boltzmann distribution factors and relative populations of conformers are given in Tables 8.2 and 8.3. As shall be demonstrated below, the assumption of the Boltzmann distribution does not always provide the ideal simulation of transition intensities.

\subsubsection{Degeneracy of Each Conformer}

This is equal to the number of enantiomers of each conformer. Conformers that belong to the $C_{\mathrm{s}}$ point group have a single conformation. For other conformers, belonging to the $C_{1}$ point group, there are two equivalent conformations that are not optically distinguishable. The weights for the latter conformers are therefore doubled in spectral simulation. The degrees of the degeneracy of MAOO conformers are given in Tables 8.2 and 8.3.

\subsubsection{Oscillator Strength of the Electronic Transition for each Conformer}


$\tilde{A} \leftarrow \tilde{X}$ transition of peroxy radicals is analogous to the triply forbidden $\mathrm{a}^{1} \Delta_{g}-\tilde{X}^{3} \Sigma_{g}^{-}$ transition of $\mathrm{O}_{2}$. Due to the nature of the forbidden transition, TD-DFT calculated oscillator strengths are not reliable. Because the electron promotion is localized to the $\mathrm{OO}$ moiety and the influence of the molecular conformation is expected to be insignificant, oscillator strengths of different conformers of MAOO are all set to unity in the spectral simulation.

\subsubsection{Franck-Condon Factors (FCFs) of the Vibronic Transitions}

FCFs for vibronic transitions were calculated using the ezSpectrum program. ${ }^{121}$ Because the electron promotion is limited to the dioxygen moiety, the most significant geometric change for the $\tilde{A} \leftarrow \tilde{X}$ transition is the elongation of the OO bond (see Table 8.4). Therefore, only the origin ( $v^{\prime}=0$ to $\left.v^{\prime \prime}=0\right)$ transitions and transitions to $\tilde{A}$-state vibrational levels with OO-stretch characters have considerable FCFs, as confirmed by calculations using ezSpectrum.

Relative transition intensities of all conformers are calculated as products of the factors listed above. To simulate the experimental spectra, several line-broadening mechanisms need to be considered, including the linewidth of the light source $(\sim 1 \mathrm{GHz})$, Doppler broadening (300 MHz), and pressure broadening (on the order of $1 \mathrm{GHz}$ under the current experimental conditions). With these line-broadenings, the rotational structure of MAOO radicals cannot be resolved, and only rotational contours are expected in the experimental spectra. However, these mechanisms are not sufficient to explain the absence of any narrow features in the experimental spectra attributable to MAOO (see below). The lack of narrow features in the experimental CRD spectra can be explained by lifetime 
broadening, which was detected in previous CRD spectra of the allyl peroxy radical. ${ }^{72}$ Due to the weak $\mathrm{CO}$ bond, the $\tilde{A}$ state of allyl peroxy is expected to overlap with the dissociative continuum of the $\tilde{X}$ state, which leads to predissociation and short lifetime of $\tilde{A}$-state energy levels. Spectral simulation using an asymmetric rotor model suggests that lifetime broadening on the order of $1 \mathrm{~cm}^{-1}$ would remove remaining features in the rotational contours of MAOO, which span more than $10 \mathrm{~cm}^{-1}$. Therefore, instead of adopting simulated rotational contours, calculated transition intensities are convoluted by a Gaussian line shape with a full width at half maximum (FWHM) of $15 \mathrm{~cm}^{-1}$ in the present work. Simulated spectra of all produced conformers are superposed to reproduce the roomtemperature CRD spectra.

\subsubsection{Simulation of CRD Spectrum of 2-MAOO}

We first compare the experimental and simulated spectra of 2-MAOO (Figure 8.5) as it was separated from the other two isomers in free radical production. The lowest-frequency and strongest peak at $\sim 7640 \mathrm{~cm}^{-1}$ in the experimental spectrum is assigned to the $\tilde{A} \leftarrow \tilde{X}$ origin. However, TD-DFT calculations overestimate the electronic transition frequencies. A scaling factor of 0.887 is therefore applied to calculated electronic transition frequencies of all conformers to match the experimental and simulated spectra. Simulated spectra of all conformers of 2-MAOO are weighted by their calculated relative populations and combined to generate the overall spectrum of 2-MAOO (thick line in Figure 8.5b). (See Figure 8.17). The simulation underestimates the transition intensity of the lowest-energy conformer (TG). Such discrepancy between the experimental and simulated intensities may be attributed to inaccuracy in the calculation of FCFs and/or to the non-Boltzmann 
distribution of different conformers. Figure 8.6 compares the experimental spectrum to simulations assuming Boltzmann distribution at different temperatures. Simulation with $T=50 \mathrm{~K}$ best reproduces the experimental spectrum.

The spectral simulation predicts a strong transition to the $v=1$ level of the OO stretch mode. Experimentally, a strong peak $\sim 8615 \mathrm{~cm}^{-1}$ blue-shifted from the origin band was observed in the CRD spectrum and can be assigned to the OO-stretch band. The calculated $\tilde{A}$-state OO-stretch frequencies of 2-MAOO conformers are listed in Table 8.3. To better reproduce the experimental spectrum, a scaling factor of 0.9 is applied to the calculated vibrational frequencies. It should be compared to the scaling factor of $0.964 \pm 0.023$ recommended by the CCCDBD database ${ }^{107}$ for B3LYP/6-31+G(d,p) calculations, which implies that TD-DFT calculations used in the present work significantly overestimate the $\tilde{A}$ state $\mathrm{OO}$ bond strength.

\subsubsection{Simulation of CRD Spectra of 1-MAOO and 3-MAOO}

CRD spectra of 1- and 3-MAOO using 3-chloro-1-butene or 1-chloro-2-butene as a precursor are shown in Figure 8.7a and 8.7b, respectively. These two spectra using different precursors are almost identical, which confirms the expectation that allylic rearrangement is significantly fast than oxygen addition. Strong absorption with sharp features between 7300 and $7500 \mathrm{~cm}^{-1}$ is due to methyl peroxy. ${ }^{159}$ In addition, a broad and long-lived baseline absorption is present in both spectra. It is attributed to unidentified stable photolysis products. 
Simulated spectra of all conformers of each isomer are combined to generate spectra of the isomers (thick lines in Figure 8.7c and 8.7d) (See Figures 8.18 and 8.19 for details.) By matching the calculated $\tilde{A} \leftarrow \tilde{X}$ adiabatic transition frequencies to the strong origin bands in the experimental spectra at $7610 \mathrm{~cm}^{-1}(1-\mathrm{MAOO})$ and $7810 \mathrm{~cm}^{-1}$ (3-MAOO), electronic scaling factors are determined to be 0.879 and 0.887 for 1- and 3-MAOO, respectively. The OO-stretch region of the experimental spectra is contaminated by strong precursor absorption (See Figure 8.20). Therefore, OO-stretch bands of 1- and 3MAOO were not observed. Nevertheless, the same vibrational scaling factor as 2MAOO (0.9) is used in the simulation of the spectrum of 1- and 3-MAOO radicals.

In simulating the overall spectra of 1- and 3-MAOO isomers (Figure 8.7e), the relative populations of 1-MAOO :3-MAOO is set to $52: 48$, the calculated ratio of spin densities on the terminal and non-terminal positions. The simulated spectrum reproduces the experimental one qualitatively. In Figure 8.21 , the population ratio is adjusted to demonstrate its effect on the overall spectrum. Although an accurate determination of the population ratio from the experimental spectrum is not possible due to the existence of the broad baseline and interference of the $\mathrm{CH}_{3} \mathrm{OO}$ absorption, the experimental CRD spectrum confirms that populations of the 1- and 3-MAOO radicals produced in the present experiment are comparable to each other.

\subsection{Conclusions}

Room-temperature CRD spectra of $\tilde{A} \leftarrow \tilde{X}$ electronic transitions of all three isomers of the MAOO radical have been recorded. The free radicals were produced in a $193 \mathrm{~nm}$ photolysis of selected methyl-substituted allyl chlorides, including 1-chloro-2-butene, 3- 
chloro-2-methyl-1 propene, and 3-chloro-1-butene, in the presence of $\mathrm{O}_{2}$. The vibronic structure of experimentally observed spectra was simulated using calculated electronic transition frequencies, vibrational frequencies, and FCFs. When 1-chloro-2-butene or 3chloro-1-butene is used as a precursor, either of the two resonance-stabilized structures of 1-MA $\left(\mathrm{CH}_{3} \mathrm{CH}=\mathrm{CHCH}_{2} \cdot \leftrightarrow \mathrm{CH}_{3} \mathrm{CH} \cdot \mathrm{CH}=\mathrm{CH}_{2}\right)$ is produced in the photolysis. The subsequent allylic rearrangement and interconversion between the two resonance structures are significantly faster than oxygen addition that forms the peroxy radicals, resulting in identical peroxy spectra using these two precursors. Spin density calculations and PES scans suggest that the branching ratio between the terminal and non-terminal oxygen addition is close to unity, which is confirmed in simulating the overall spectra of 1- and 3-MAOO radicals.

The experimental investigation reported in the present paper is the first attempt at the spectrum of an important and intriguing free radical. In order to reduce spectral congestion and achieve better resolution, one may produce the MAOO radicals with the pulsed discharge of stable precursors in a slit-jet supersonic expansion in the presence of oxygen. ${ }^{104,160}$ Also, it would be beneficial to use narrow linewidth near-IR sources, e.g., the first-order Stokes of a Ti:Sapphire amplifier. ${ }^{161}$ Construction of a high-resolution, jetcooled CRD spectroscopy apparatus is in process. 
Table 8.1. Numbers of (optically distinguishable) conformations, transition structures, and stable conformers of 1-, 2- and 3-MAOO radicals.

\begin{tabular}{|c|c|c|c|c|c|c|}
\hline \multirow{2}{*}{ isomer } & \multicolumn{3}{|c|}{ conformations } & \multirow{2}{*}{$\begin{array}{c}\text { optically } \\
\text { distinguishable } \\
\text { conformations }\end{array}$} & \multirow{2}{*}{$\begin{array}{l}\text { transition } \\
\text { structures }\end{array}$} & \multirow{2}{*}{$\begin{array}{c}\text { stable } \\
\text { conformers }\end{array}$} \\
\hline & $C_{s}$ & $C_{1}$ & total & & & \\
\hline 1-MAOO & 2 & 16 & 18 & 10 & 2 & 8 \\
\hline 2-MAOO & 1 & 8 & 9 & 5 & 0 & 5 \\
\hline 3-MAOO ${ }^{\circ}$ & 0 & 18 & 18 & 9 & 0 & 9 \\
\hline
\end{tabular}


Table 8.2. Relative energies, Boltzmann distribution factors, and relative populations of 1and 3-MAOO conformers. $\Delta E^{\tilde{A}-\tilde{X}}$ is the adiabatic transition frequency between the $\tilde{X}$ and $\tilde{A}$ states. $v_{\mathrm{OO}}(\tilde{A})$ is the $\tilde{A}$-state $\mathrm{OO}$ stretch frequency.

\begin{tabular}{|c|c|c|c|c|c|c|c|}
\hline isomer & conformer & $\begin{array}{c}\text { no. of } \\
\text { enantiomers }\end{array}$ & $\begin{array}{l}\text { relative energy }{ }^{\mathrm{a}} \\
\qquad\left(\mathrm{cm}^{-1}\right)\end{array}$ & $\begin{array}{c}\text { Boltzmann } \\
\text { distribution } \\
\text { factor }^{\mathrm{b}}\end{array}$ & $\begin{array}{c}\text { relative } \\
\text { population }^{\mathrm{c}} \\
(\%)\end{array}$ & $\begin{array}{l}\Delta E^{\tilde{A}-\tilde{X}} \\
\left(\mathrm{~cm}^{-1}\right)\end{array}$ & $\begin{array}{l}v_{\mathrm{OO}}(\tilde{A}) \\
\left(\mathrm{cm}^{-1}\right)\end{array}$ \\
\hline \multirow{10}{*}{ 1-MAOO ${ }^{\circ}$} & TGT & 2 & 0 & 0.41 & 21.4 & 7607 & 1012 \\
\hline & GGT & 2 & 60 & 0.31 & 16.0 & 7671 & 1013 \\
\hline & GG'T & 2 & 138 & 0.21 & 11.0 & 7594 & 1004 \\
\hline & $\mathrm{TCT}$ & 1 & 598 & 0.01 & 0.6 & 7517 & 1043 \\
\hline & GCT & 2 & 658 & 0.02 & 0.9 & 7555 & 1002 \\
\hline & TGC & 2 & 669 & 0.02 & 0.8 & 7605 & 1011 \\
\hline & GGC & 2 & 724 & 0.01 & 0.6 & 7782 & 1014 \\
\hline & $\mathrm{GG}^{\prime} \mathrm{C}$ & 2 & 787 & 0.01 & 0.5 & 7646 & 1008 \\
\hline & $\mathrm{GCC}^{\mathrm{d}}$ & 2 & & & & & \\
\hline & $\mathrm{TCC}^{\mathrm{d}}$ & 1 & & & & & \\
\hline \multirow{9}{*}{ 3-MAOO } & $\mathrm{TG}^{\prime}$ & 2 & 182 & 0.32 & 15.1 & 7801 & 1012 \\
\hline & $\mathrm{GG}^{\prime}$ & 2 & 200 & 0.29 & 13.8 & 7766 & 1012 \\
\hline & $G^{\prime} G^{\prime}$ & 2 & 372 & 0.13 & 6.1 & 7840 & 1006 \\
\hline & GG & 2 & 469 & 0.08 & 3.8 & 7876 & 1023 \\
\hline & TG & 2 & 477 & 0.08 & 3.7 & 7817 & 1014 \\
\hline & $\mathrm{TC}$ & 2 & 566 & 0.05 & 2.4 & 7723 & 1036 \\
\hline & GC & 2 & 604 & 0.04 & 2.0 & 7596 & 1022 \\
\hline & G'G & 2 & 810 & 0.02 & 0.7 & 7715 & 994 \\
\hline & $\mathrm{G}^{\prime} \mathrm{C}$ & 2 & 943 & 0.01 & 0.4 & 7635 & 999 \\
\hline
\end{tabular}

${ }^{\text {a } Z e r o-p o i n t ~ e n e r g y ~ c o r r e c t i o n s ~ i n c l u d e d . ~}$

${ }^{\mathrm{b}}$ Normalized for each isomer.

${ }^{\mathrm{c}}$ Normalized for 1- and 3-MAOO combined. The population ratio of 1-MAOO:3-

MAOO is set to 52:48. (See text).

${ }^{\mathrm{d}}$ Transition structures. 
Table 8.3. Relative energies, Boltzmann distribution factors, and relative populations of 2MAOO conformers. $\Delta E^{\tilde{A}-\tilde{X}}$ is the adiabatic transition frequency between the $\tilde{X}$ and $\tilde{A}$ states. $v_{\mathrm{OO}}(\tilde{A})$ is the $\tilde{A}$-state OO stretch frequency.

\begin{tabular}{cccccccc}
\hline isomer & conformer & $\begin{array}{c}\text { no. of } \\
\text { enantiomer }\end{array}$ & $\begin{array}{c}\text { relative } \\
\text { energy }^{\mathrm{a}} \\
\left(\mathrm{cm}^{-1}\right)\end{array}$ & $\begin{array}{c}\text { Boltzmann } \\
\text { distribution }^{\text {factor }}{ }^{\mathrm{b}}\end{array}$ & $\begin{array}{c}\text { relative } \\
\text { population } \\
(\%)\end{array}$ & $\Delta E^{\tilde{A}-\tilde{X}}\left(\mathrm{~cm}^{-1}\right)$ & $v_{\mathrm{OO}}\left(\mathrm{cm}^{-1}\right)$ \\
\hline \multirow{5}{*}{ 2-MAOO $^{\bullet}$} & TG & 2 & 0 & 0.37 & 37.5 & 7642 & 1023 \\
& GG & 2 & 52 & 0.29 & 29.3 & 7812 & 1014 \\
& G'G & 2 & 98 & 0.23 & 23.4 & 7534 & 1002 \\
& TC & 1 & 350 & 0.07 & 7.0 & 7596 & 1046 \\
& GC & 2 & 388 & 0.03 & 2.9 & 7657 & 1034 \\
\hline
\end{tabular}

a Zero-point energy corrections included.

${ }^{\mathrm{b}}$ Normalized. 
Table 8.4. Geometric parameters of conformers of 1-, 2- and 3- MAOO radicals in the $\tilde{X}$ and $\tilde{A}$ states calculated at the B3LYP/6-31+G(d,p) level of theory.

\begin{tabular}{|c|c|c|c|c|c|c|c|c|c|c|c|}
\hline \multirow{3}{*}{ isomer } & \multirow{3}{*}{ conformer } & \multicolumn{10}{|c|}{ geometric parameter } \\
\hline & & \multicolumn{2}{|c|}{$\begin{array}{l}\mathrm{O}-\mathrm{O} \\
(\AA)\end{array}$} & \multicolumn{2}{|c|}{$\begin{array}{l}\angle \mathrm{OOC} \\
\text { (deg.) }\end{array}$} & \multicolumn{2}{|c|}{$\begin{array}{c}\phi \mathrm{OOCC} \\
\text { (deg.) }\end{array}$} & \multicolumn{2}{|c|}{$\begin{array}{c}\phi \mathrm{OCCC} \\
\text { (deg.) }\end{array}$} & \multicolumn{2}{|c|}{$\begin{array}{c}\phi C C C C \\
\text { (deg.) }\end{array}$} \\
\hline & & $\tilde{X}$ & $\tilde{A}$ & $\tilde{X}$ & $\tilde{A}$ & $\tilde{X}$ & $\tilde{A}$ & $\tilde{X}$ & $\tilde{A}$ & $\tilde{X}$ & $\tilde{A}$ \\
\hline \multirow{10}{*}{ 1-MAOO } & TGT & 1.32 & 1.38 & 111 & 108 & 169 & 174 & 114 & 114 & 179 & 178 \\
\hline & GGT & 1.32 & 1.38 & 112 & 109 & 82 & 72 & 116 & 114 & 180 & 178 \\
\hline & GG'T & 1.32 & 1.38 & 112 & 110 & 77 & 70 & -114 & -124 & -180 & -179 \\
\hline & TCT & 1.32 & 1.38 & 111 & 108 & 180 & 180 & 0 & 0 & 180 & 180 \\
\hline & GCT & 1.33 & 1.38 & 112 & 110 & 89 & 71 & -8 & 0 & 180 & 180 \\
\hline & TGC & 1.32 & 1.38 & 111 & 108 & -172 & -175 & -113 & -112 & 0 & 1 \\
\hline & GGC & 1.32 & 1.38 & 112 & 109 & 80 & 70 & 119 & 116 & 0 & -1 \\
\hline & $\mathrm{GG}^{\prime} \mathrm{C}$ & 1.32 & 1.38 & 112 & 110 & 78 & 71 & -112 & -125 & 0 & 2 \\
\hline & $\mathrm{TCC}^{\mathrm{a}}$ & 1.32 & 1.38 & 111 & 108 & 180 & 180 & 0 & 0 & 0 & 0 \\
\hline & $\mathrm{GCC}^{\mathrm{a}, \mathrm{b}}$ & & & & & & & & & & \\
\hline \multirow{5}{*}{ 2-MAOO } & TG & 1.32 & 1.38 & 112 & 108 & 168 & 176 & 117 & 121 & & \\
\hline & GG & 1.32 & 1.38 & 112 & 111 & 95 & 75 & 114 & 109 & & \\
\hline & G'G & 1.32 & 1.38 & 112 & 110 & -79 & -67 & 120 & 131 & & \\
\hline & $\mathrm{TC}$ & 1.32 & 1.38 & 111 & 108 & 180 & 180 & 0 & 0 & & \\
\hline & $\mathrm{GC}$ & 1.33 & 1.39 & 112 & 110 & 90 & 74 & -5 & 1 & & \\
\hline \multirow{9}{*}{ 3-MAOO } & TG' & 1.32 & 1.38 & 112 & 110 & 167 & 175 & -118 & -119 & & \\
\hline & $\mathrm{GG}^{\prime}$ & 1.32 & 1.38 & 112 & 110 & 77 & 66 & -116 & -125 & & \\
\hline & $G^{\prime} G^{\prime}$ & 1.32 & 1.38 & 113 & 112 & -65 & -68 & -118 & -117 & & \\
\hline & GG & 1.32 & 1.38 & 112 & 110 & 74 & 70 & 130 & 133 & & \\
\hline & TG & 1.32 & 1.38 & 112 & 110 & 158 & 164 & 129 & 129 & & \\
\hline & $\mathrm{TC}$ & 1.32 & 1.38 & 112 & 109 & 160 & 168 & -2 & -2 & & \\
\hline & GC & 1.32 & 1.38 & 112 & 111 & 88 & 70 & -3 & 6 & & \\
\hline & $G^{\prime} G$ & 1.32 & 1.38 & 112 & 111 & -65 & -66 & 134 & 139 & & \\
\hline & $\mathrm{G}^{\prime} \mathrm{C}$ & 1.32 & 1.38 & 113 & 113 & -77 & -70 & 6 & 2 & & \\
\hline
\end{tabular}

a Transition structures.

b Converges to the GGC conformer upon geometry optimization. 


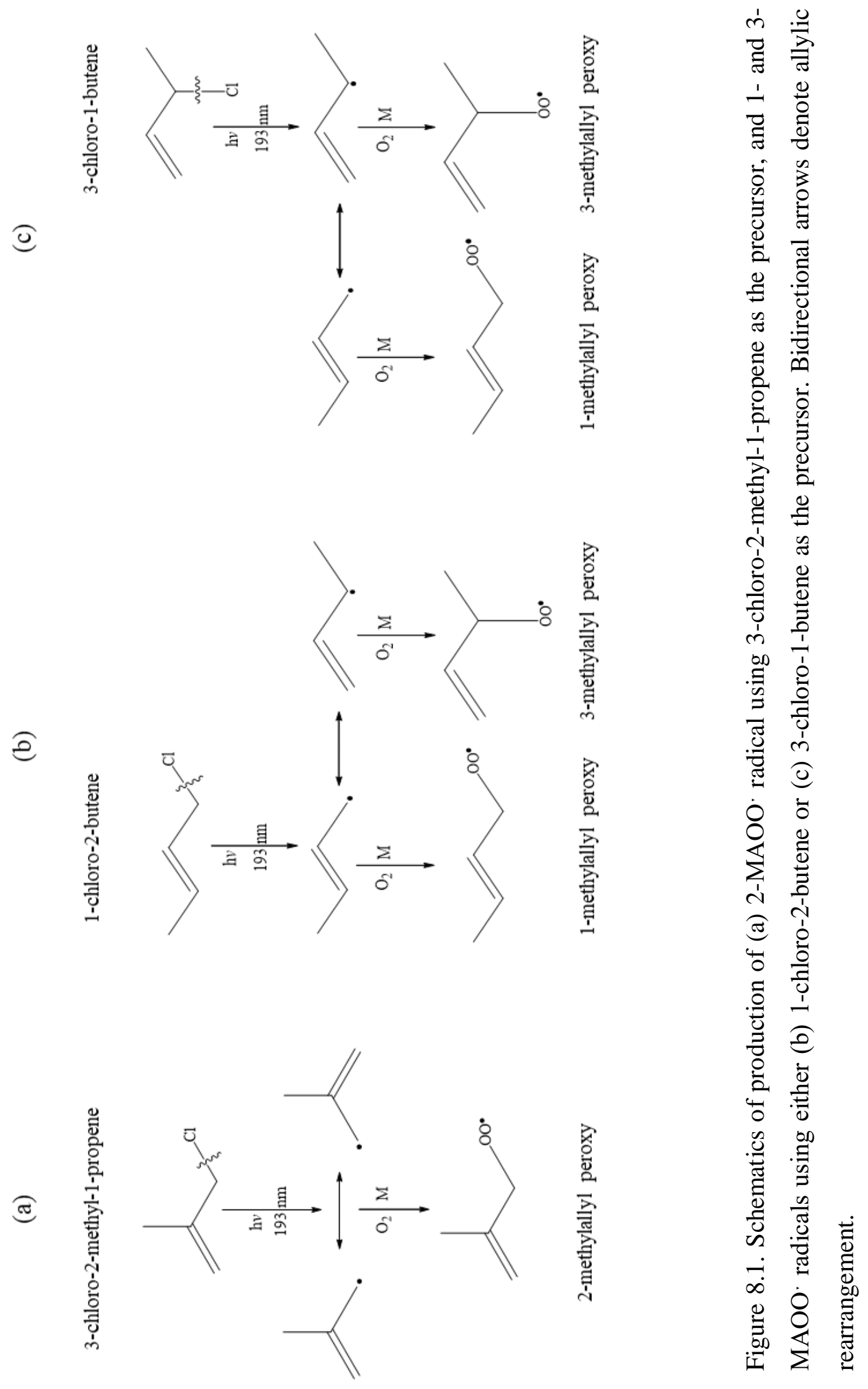




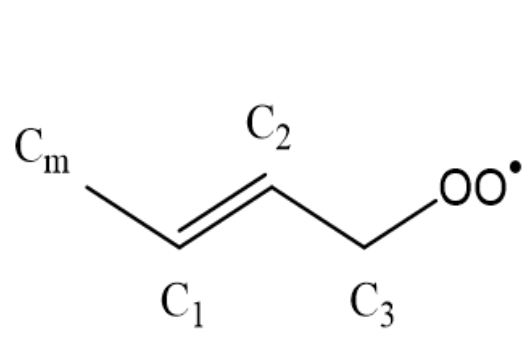

1-methylallyl Peroxy

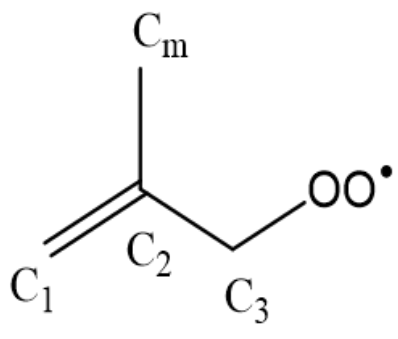

2-methylallyl Peroxy

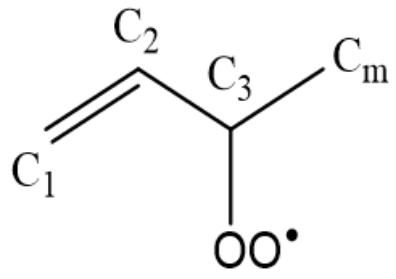

3-methylallyl Peroxy

Figure 8.2. Schematics of 1-, 2- and 3-MAOO radicals showing the convention used to label carbon atoms in the present work. 
(a)

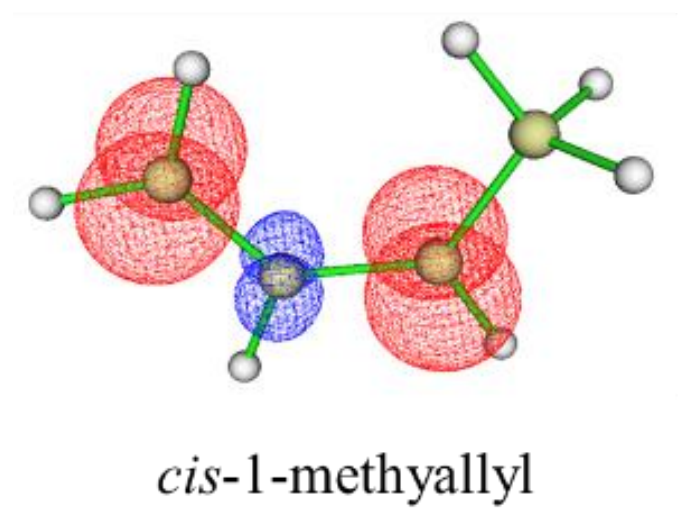

(b)

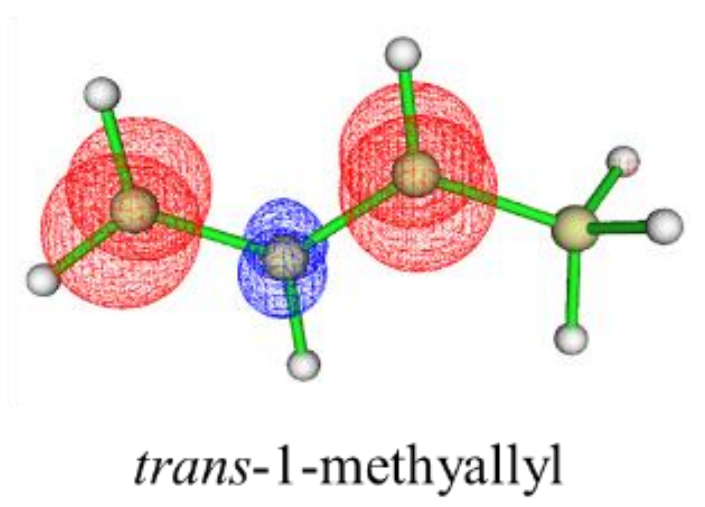

Figure 8.3. Plots of spin densities of $\tilde{X}$-state (a) cis-1-methylallyl and (b) trans-1methylallyl radicals calculated at the B3LYP/6-31+(d,p) level of theory. The regions in red and purple meshes represent excess $\alpha$ spin and $\beta$ spin, respectively. 


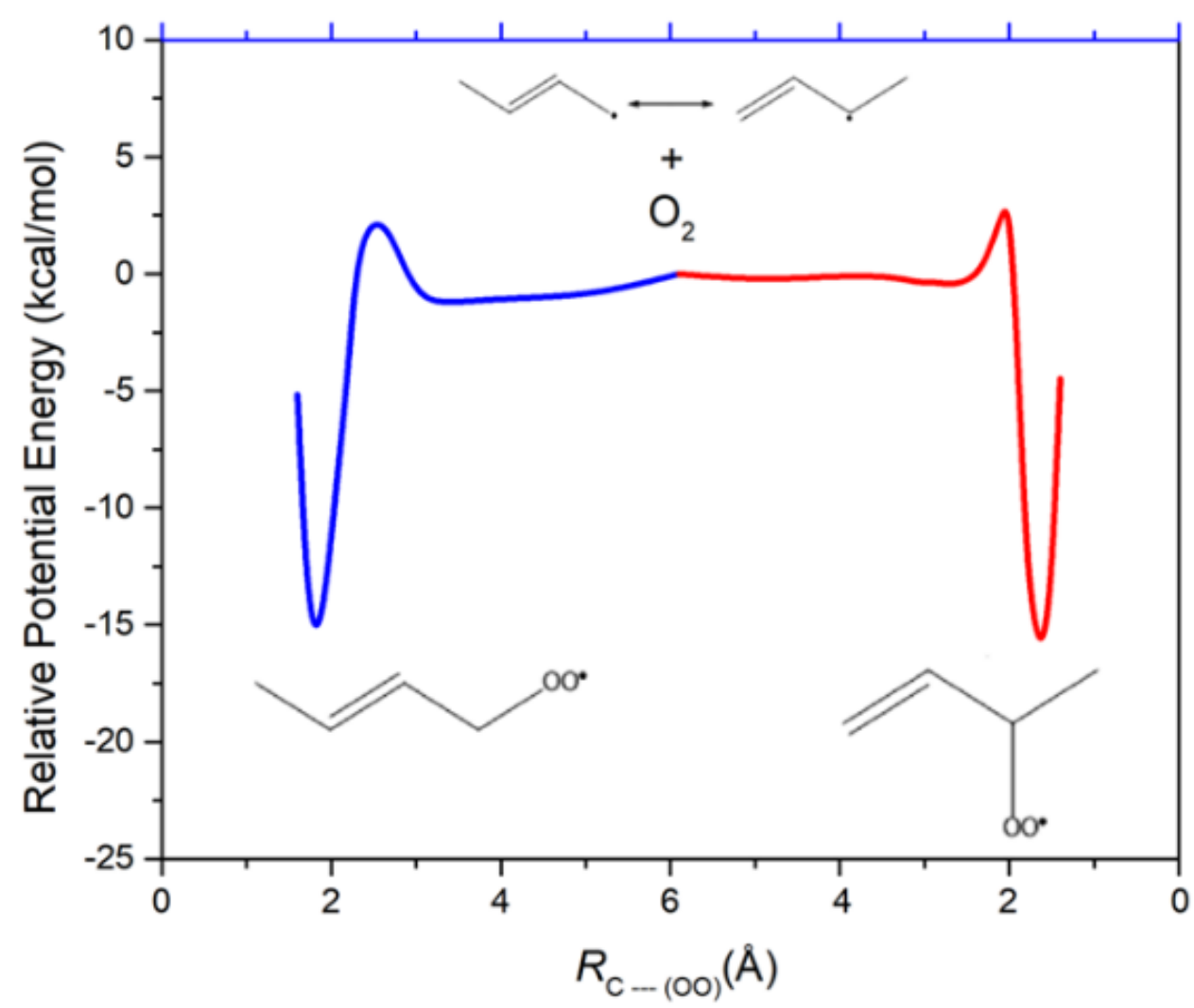

Figure 8.4. PES scans for production of 1- and 3-MAOO isomers along the reaction coordinate of oxygen addition. $R_{\mathrm{C} . . .(\mathrm{OO})}$ is the distance between the $\mathrm{C}_{3}$ atom and the center of mass of the $\mathrm{OO}$ moiety. 


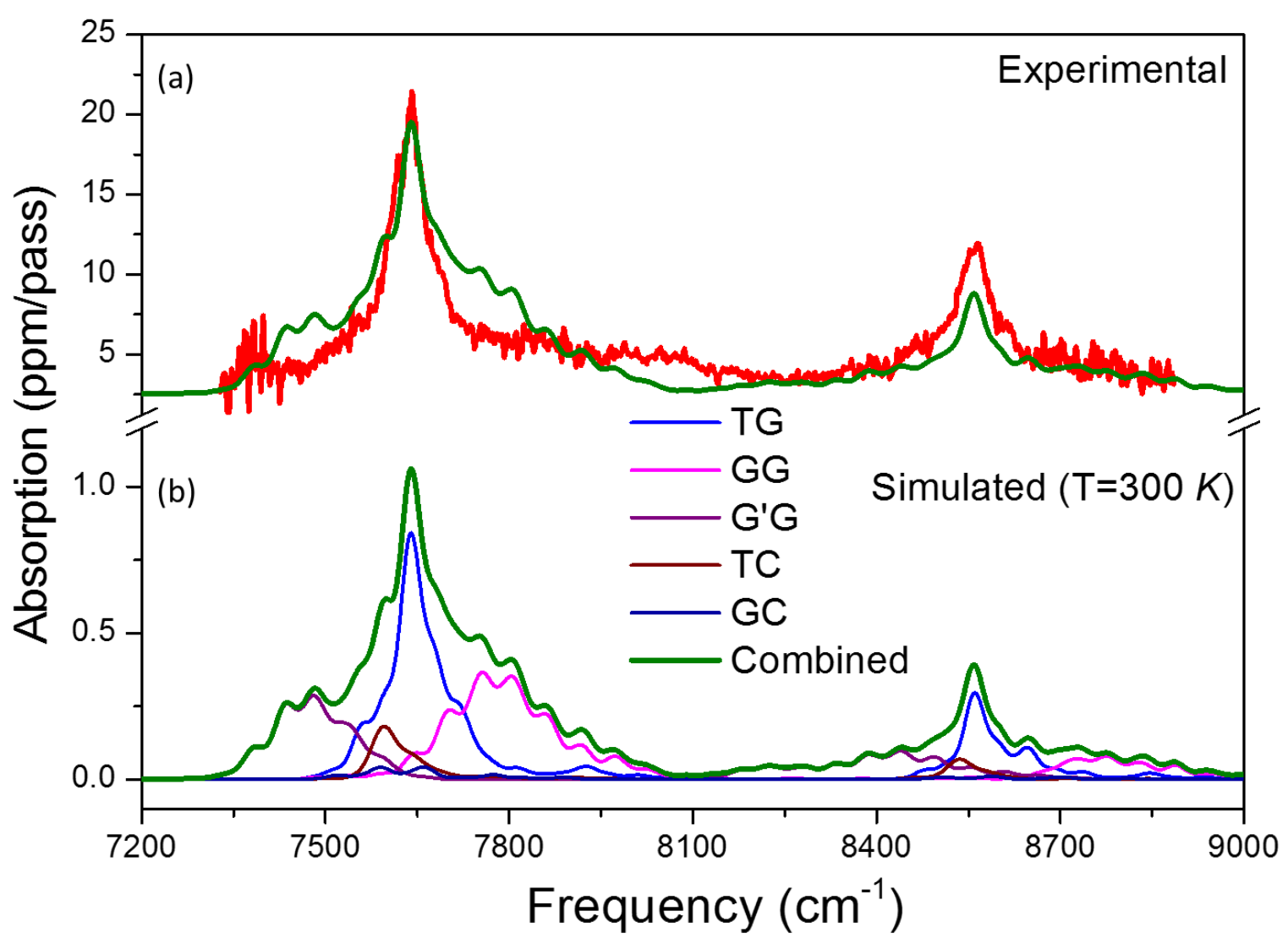

Figure 8.5. (a) Experimental CRD spectrum of the 2-MAOO radical (red) in comparison with simulation at the temperature of $300 \mathrm{~K}$ (green). Sharp peaks between 7300 and $7500 \mathrm{~cm}^{-1}$ in the experimental spectrum are due to residual water absorption. (b) Simulated spectrum of 2-MAOO. Thin lines are simulated spectra of individual conformers weighted by relative populations. The thick green line is superposition of simulated spectra of all conformers. 


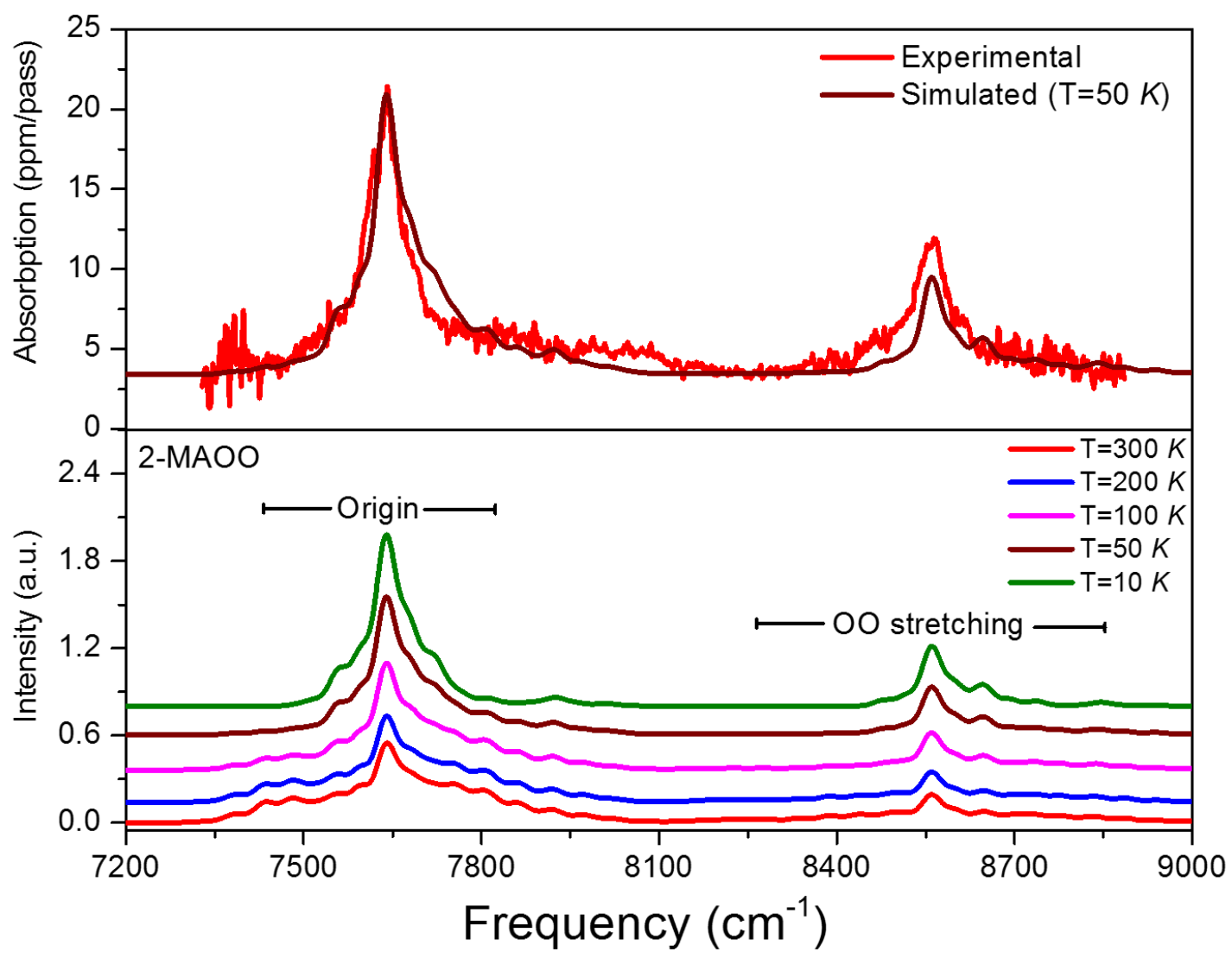

Figure 8.6. (a) Experimental CRD spectrum of the 2-MAOO radical (red) in comparison with simulation at the temperature of $50 K$ (purple). (b) Simulated spectra of 2-MAOO at different temperatures. Overall spectra of 2-MAOO are calculated as summation of spectra of all conformers weighted by their relative populations assuming the Boltzmann distribution (not shown in the figure). All spectra are offset for clarity. The simulation with $\mathrm{T}=50 \mathrm{~K}$ (purple) reproduces the experimental spectrum the best. 


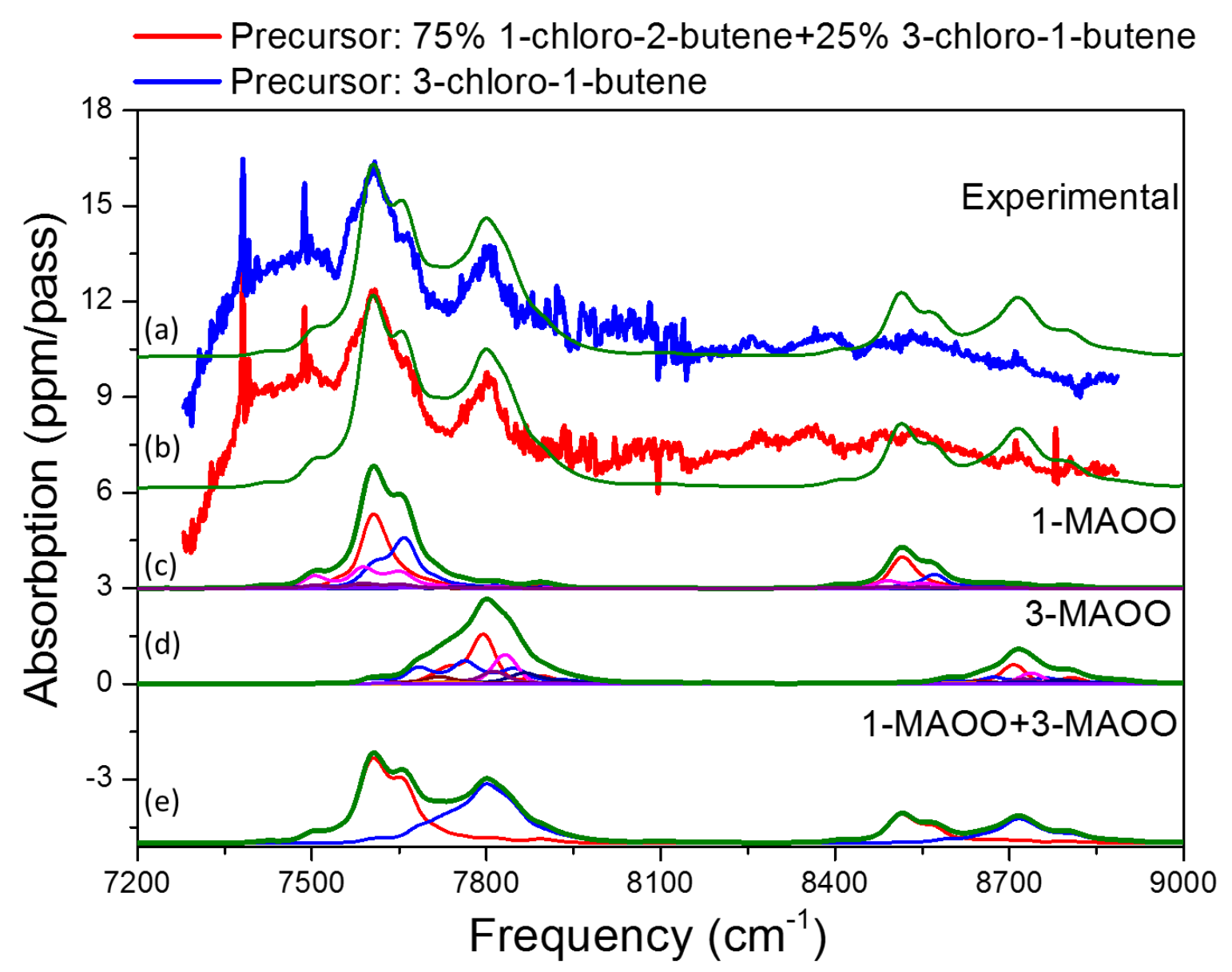

Figure 8.7. CRD spectrum of the 1- MAOO and 3- MAOO radicals using either (a) 3chloro-1-butene (blue) or (b) 75\% 1-chloro-2-butene and 25\% 3-chloro-1-butene mixture (red) as precursors. Strong absorption with sharp features between 7300 and $7500 \mathrm{~cm}^{-1}$ is due to methyl peroxy. Experimental spectra are compared with simulated overall spectra of 1- and 3-MAOO radicals (green). (c) Simulated spectrum of 1MAOO . (d) Simulated spectrum of 3-MAOO. Thin lines in (c) and (d) are simulated spectra of individual conformers weighted by populations, while the thick green line is their superposition. (e) Superposition of the simulated spectra of both isomers (green) with weights of $52 \%$ for 1-MAOO (red) and $48 \%$ for 3 -MAOO (blue). 


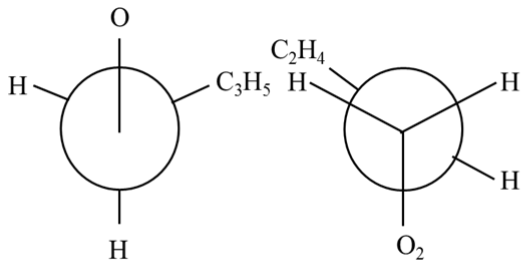<smiles>CCCCCCCCCCCCCCCCCCC</smiles>

$\mathrm{H}$

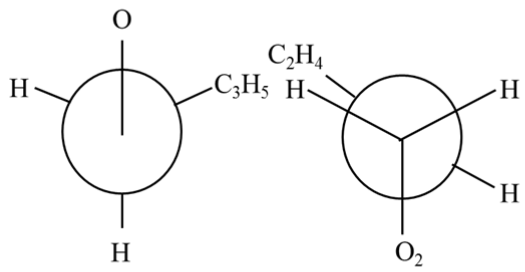

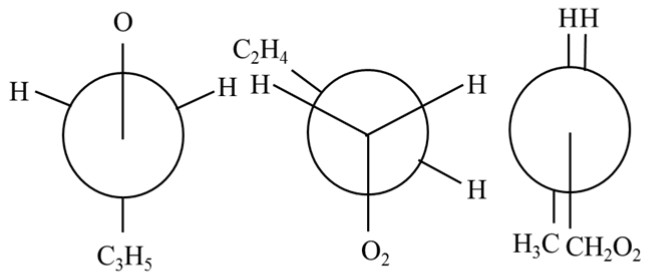<smiles>CCCCCCCCC1CCCCCC(O)C1</smiles>

$\mathrm{C}_{3} \mathrm{H}_{5}$
GGC<smiles>CCOC1CC2CC3CCC(C2)CC1CC3</smiles>

GCT

GGT

TGC
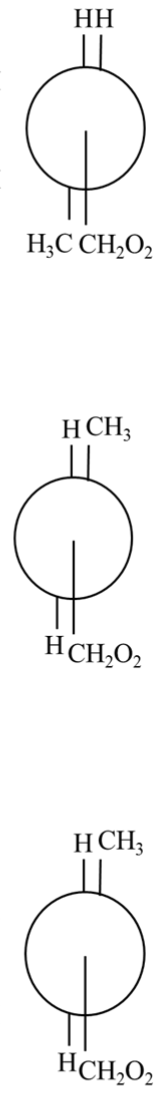

$\mathrm{CH}_{2} \mathrm{O}_{2}$

$$
\mathrm{H}_{3} \mathrm{CCH}_{2} \mathrm{O}_{2}
$$

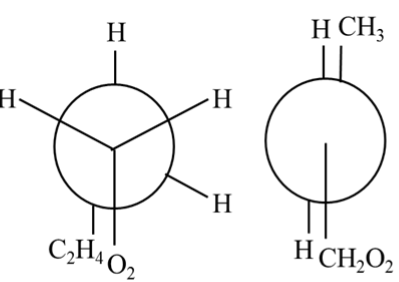

TCT

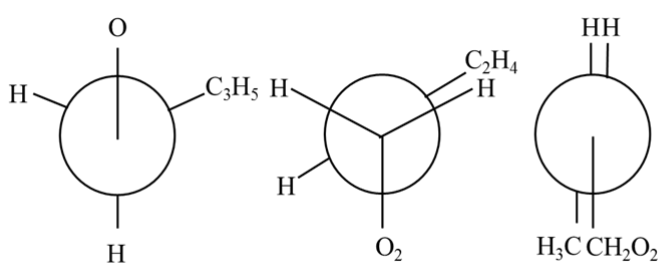

GG'C<smiles>CCCCCCCC1CCCCCCCC1O</smiles>

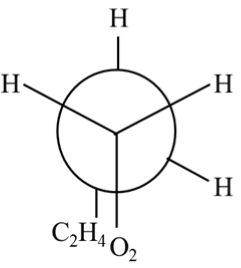<smiles>C=C1CCCC=CCCCC1CO</smiles>

GCC

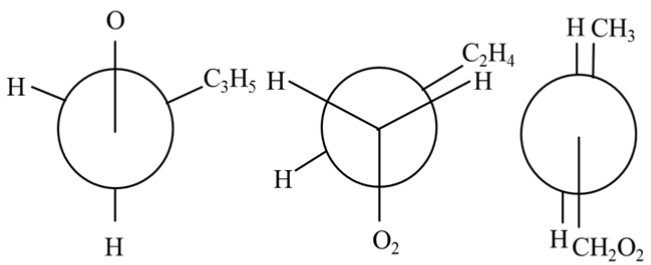

GG'T

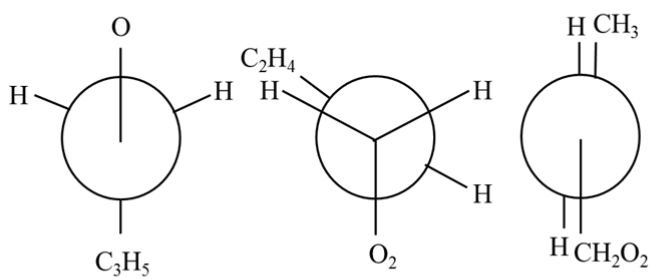

TGT

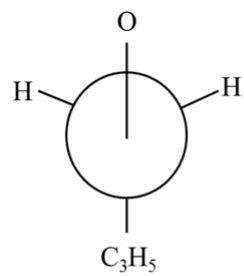<smiles>CCOC1CC2CCC3CCC(C2)CC1C3</smiles><smiles>C=C1CCCC=CCCCC1CO</smiles>

TCC

Figure 8.8. Newman projections of 1-MAOO conformers. 


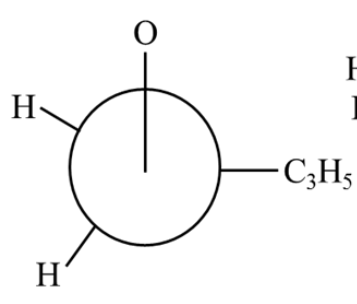

GG
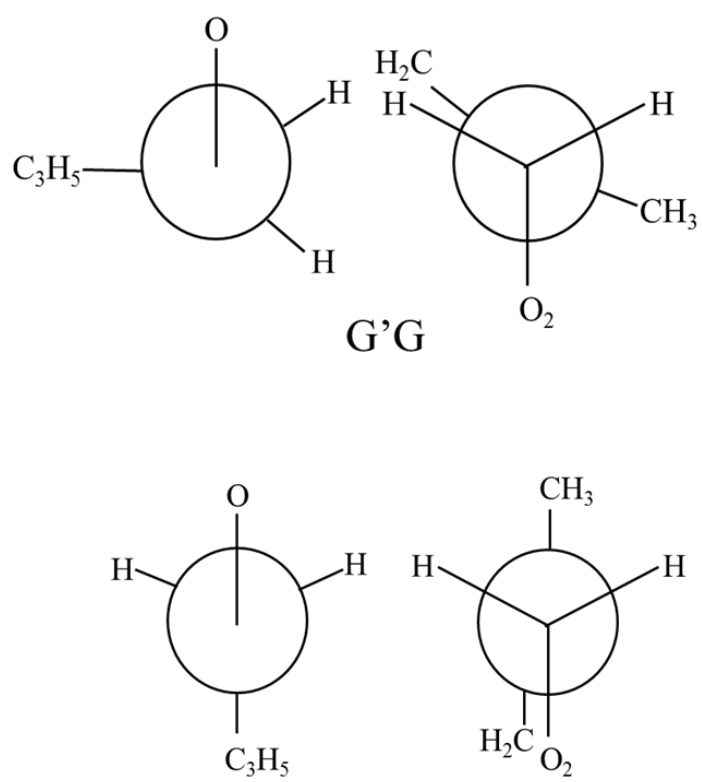

$\mathrm{TC}$

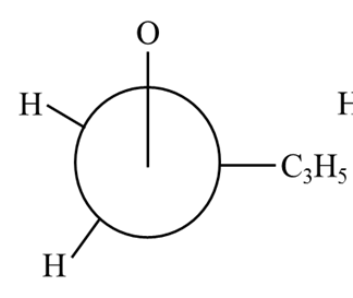

GC
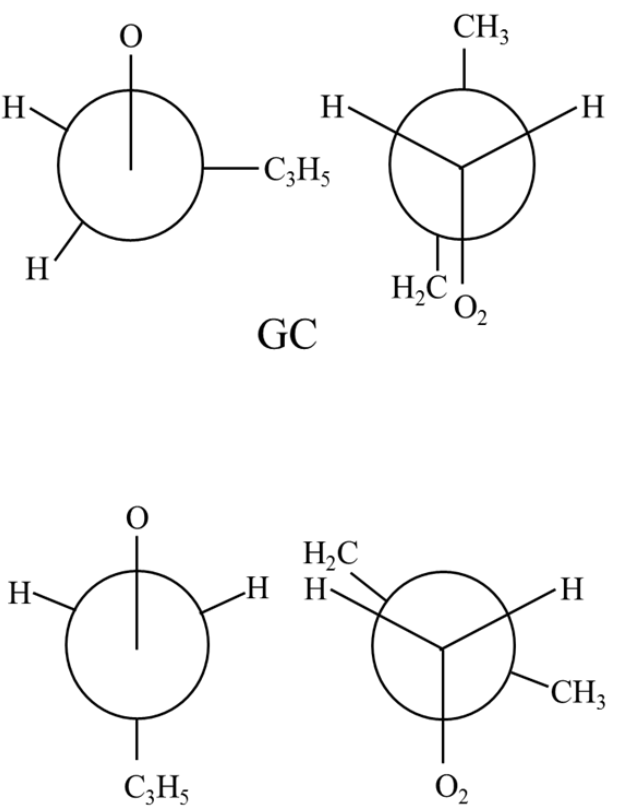

TG

Figure 8.9. Newman projections of 2-MAOO conformers. 


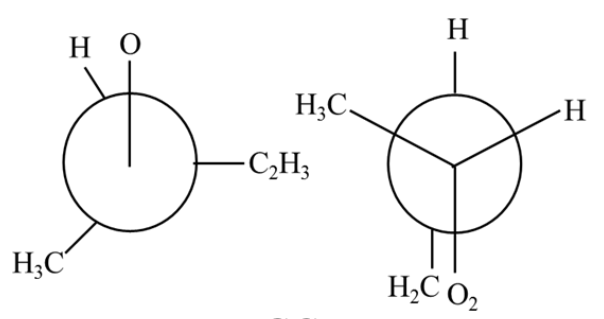

GC

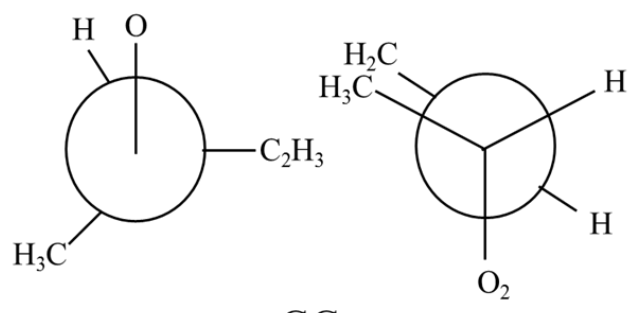

GG

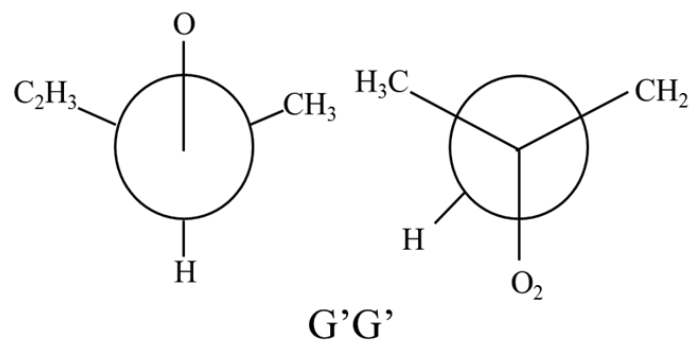

$\overbrace{\mathrm{C}_{2} \mathrm{H}_{3}}^{\mathrm{H}}$<smiles>CC1C2CCCC1(C)C(=O)CC2</smiles>

$\mathrm{TC}$

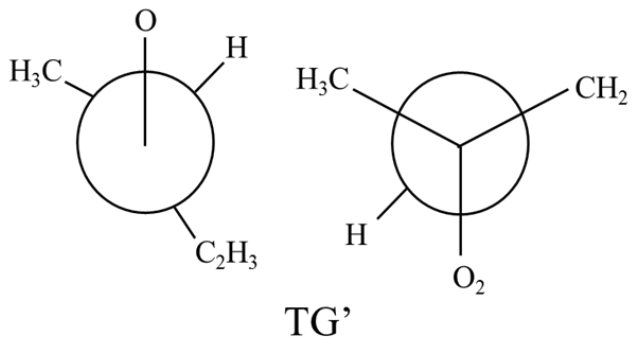

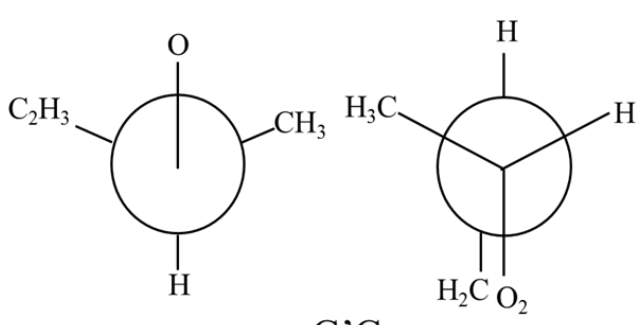

G'C

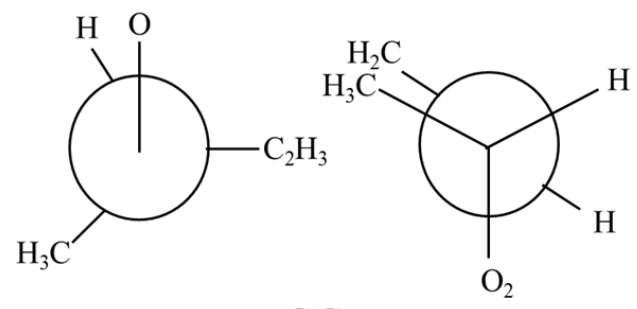

GG

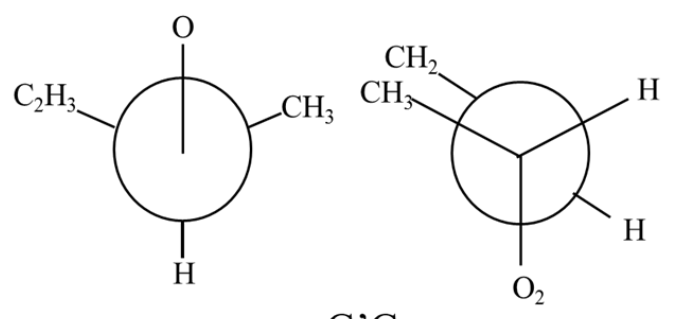

G'G

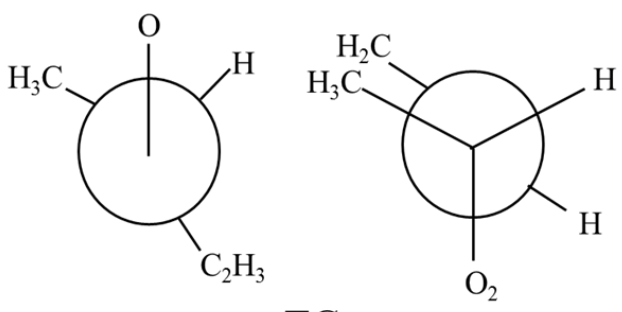

TG

Figure 8.10. Newman projections of 3-MAOO conformers. 


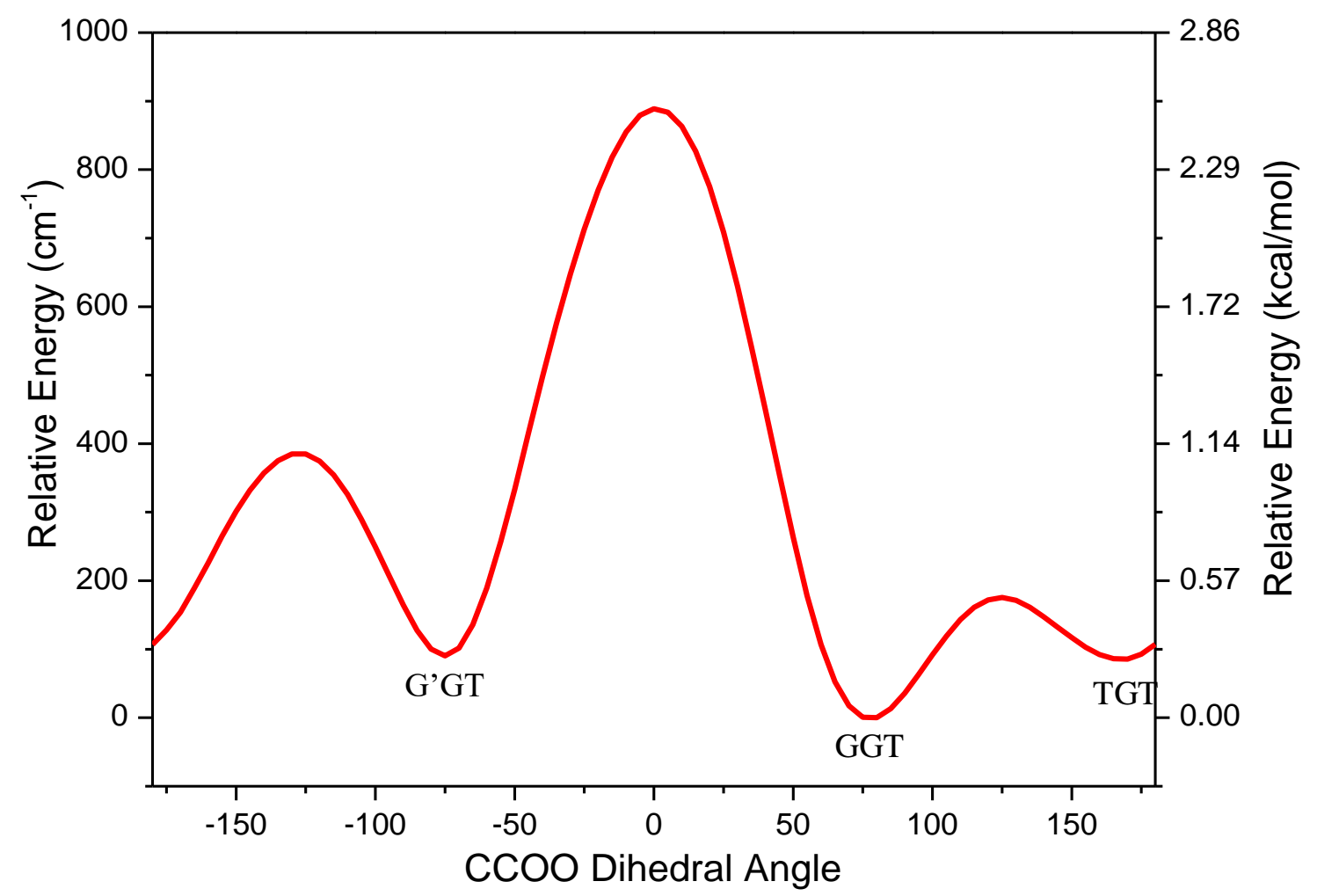

Figure 8.11. A scan of the potential energy surface of 1-MAOO along the coordinate of the CCOO dihedral angle. Molecular geometry is optimized at each step of the scan. 


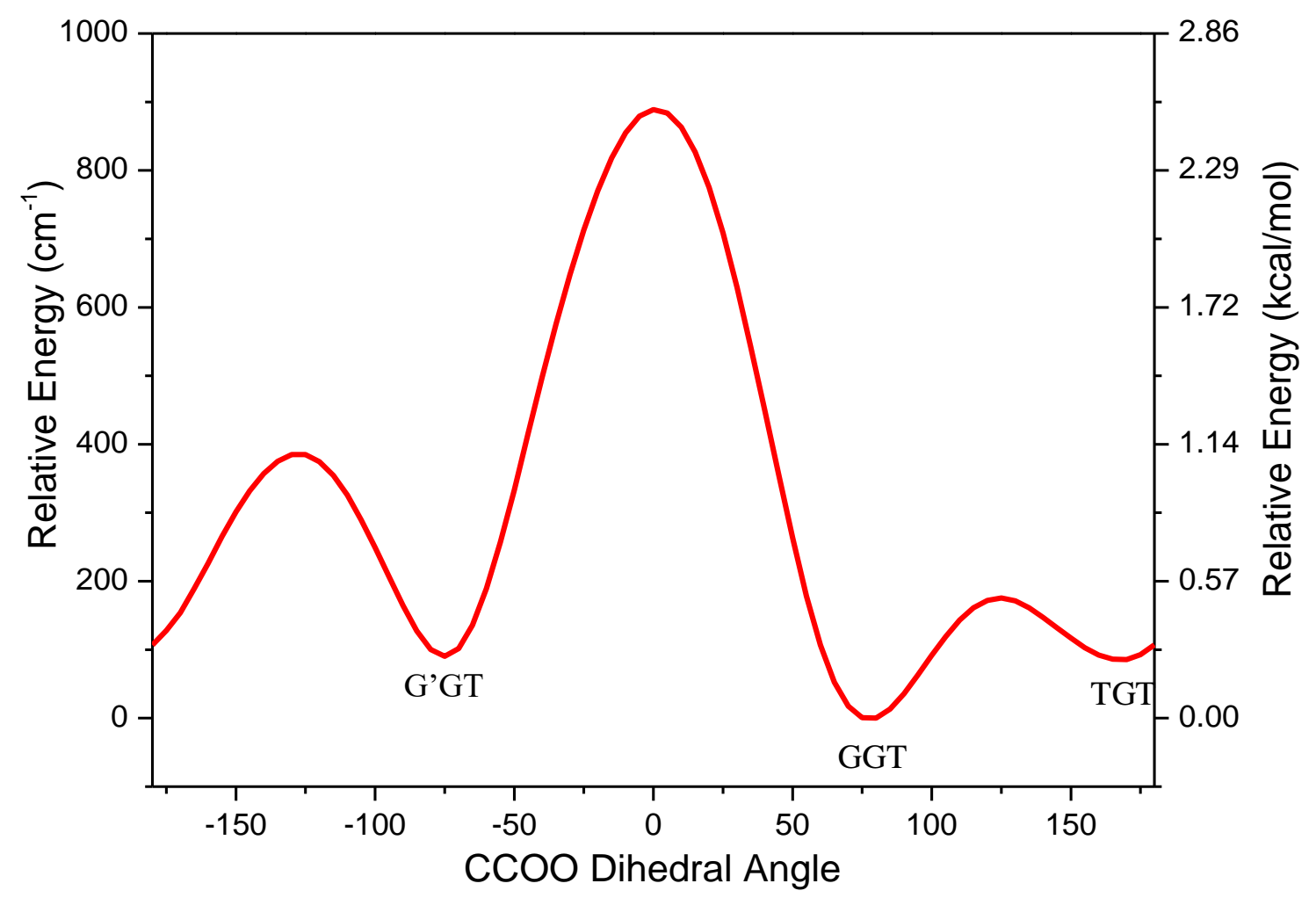

Figure 8.12. A scan of the potential energy surface of T 1-MAOO along the coordinate of the CCCO dihedral angle. Molecular geometry is optimized at each step of the scan. 


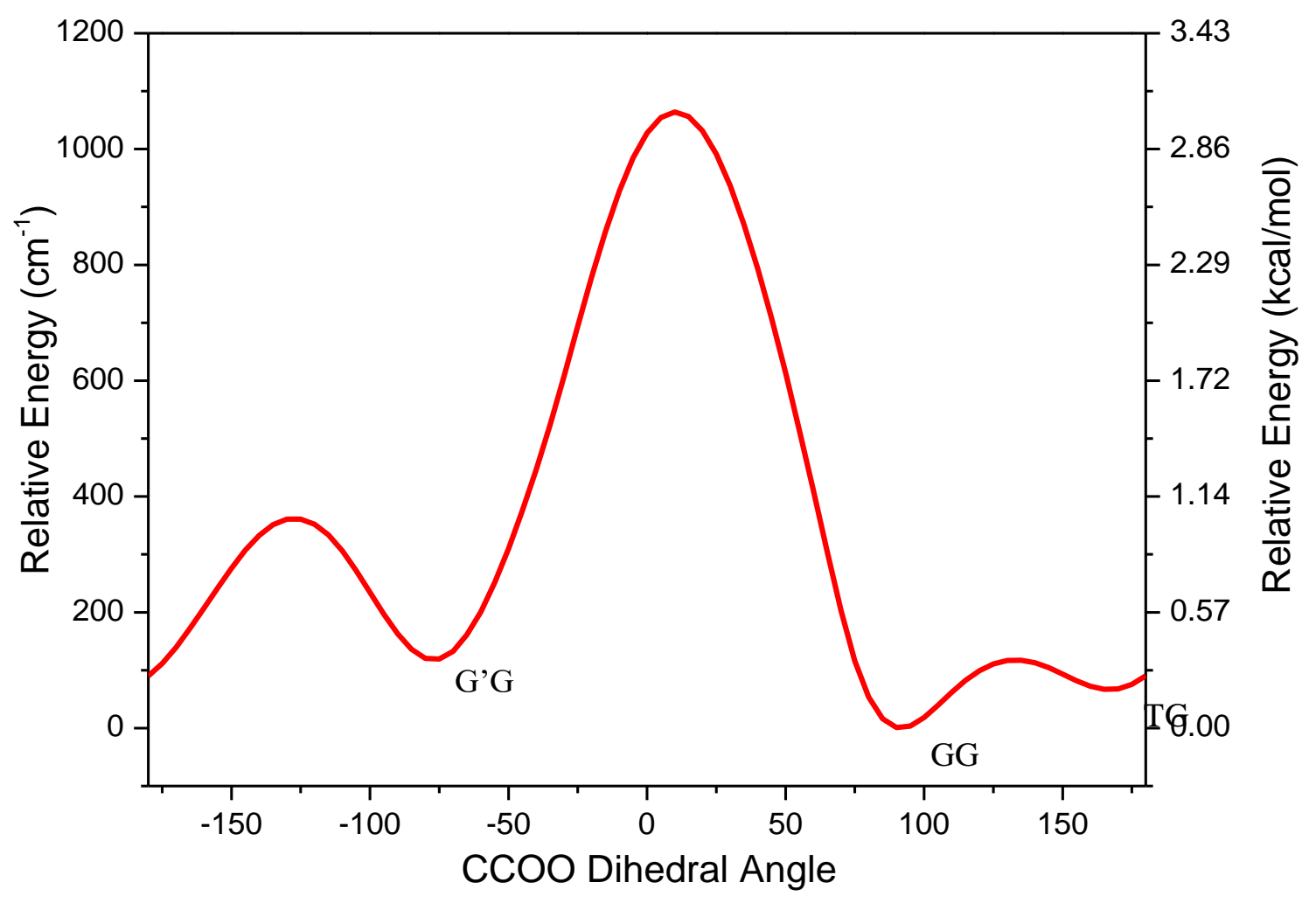

Figure 8.13. A scan of the potential energy surface of 2-MAOO along the coordinate of the $\mathrm{CCOO}$ dihedral angle. Molecular geometry is optimized at each step of the scan. 


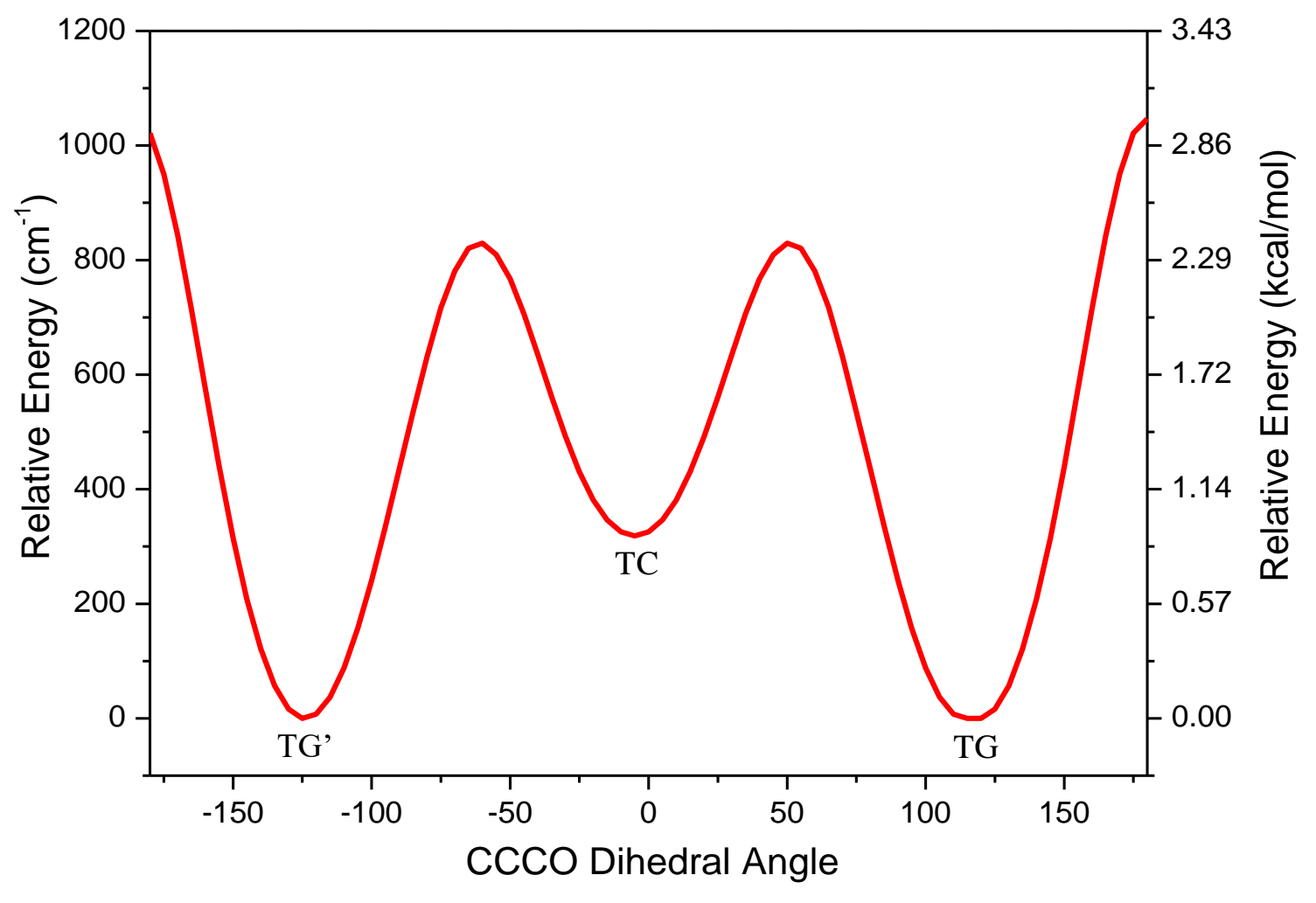

Figure 8.14 A scan of the potential energy surface of $2-\mathrm{MAOO}^{\circ}$ along the coordinate of the CCCO dihedral angle. Molecular geometry is optimized at each step of the scan. 


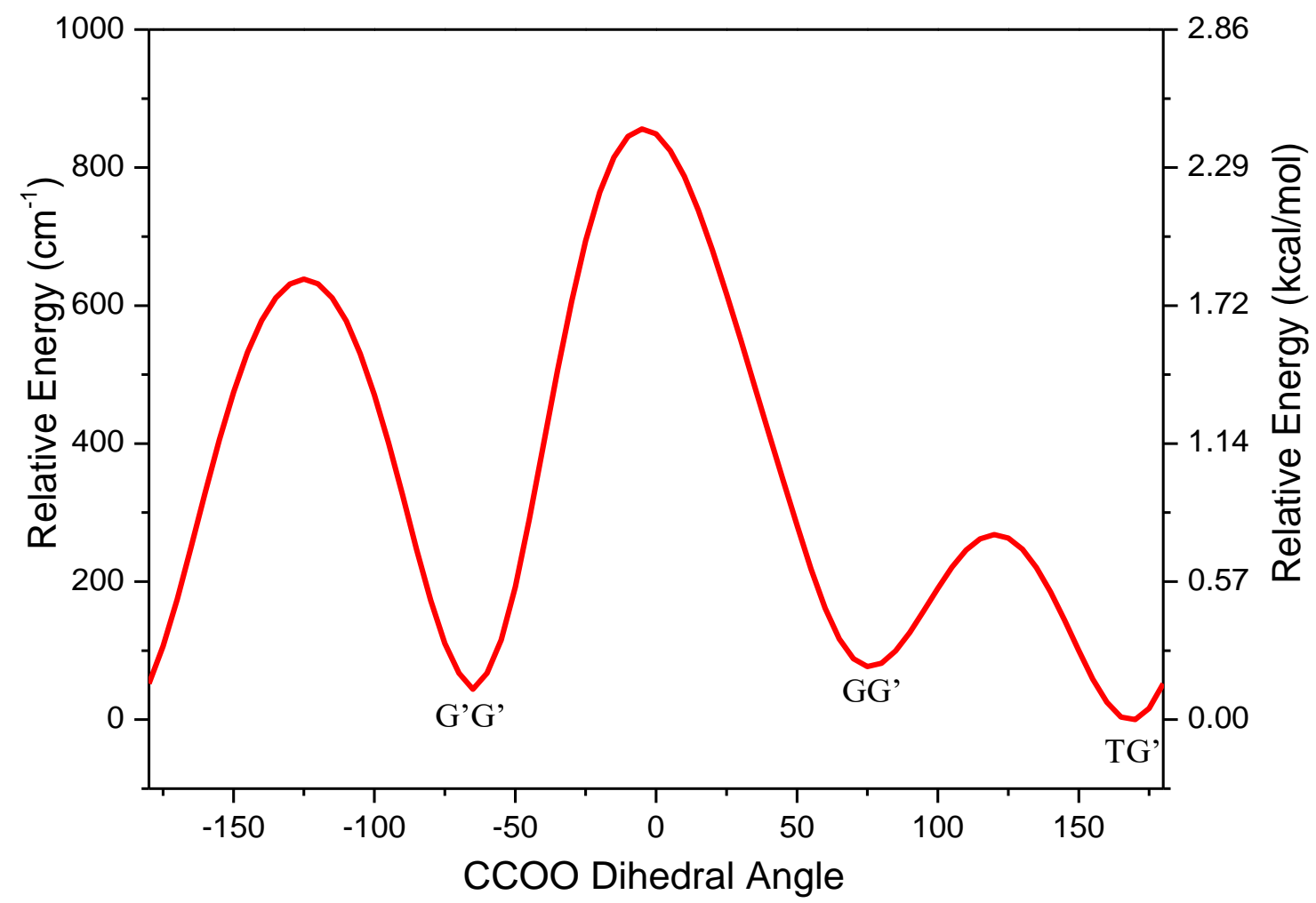

Figure 8.15. A scan of the potential energy surface of 3-MAOO along the coordinate of the CCOO dihedral angle. Molecular geometry is optimized at each step of the scan. 


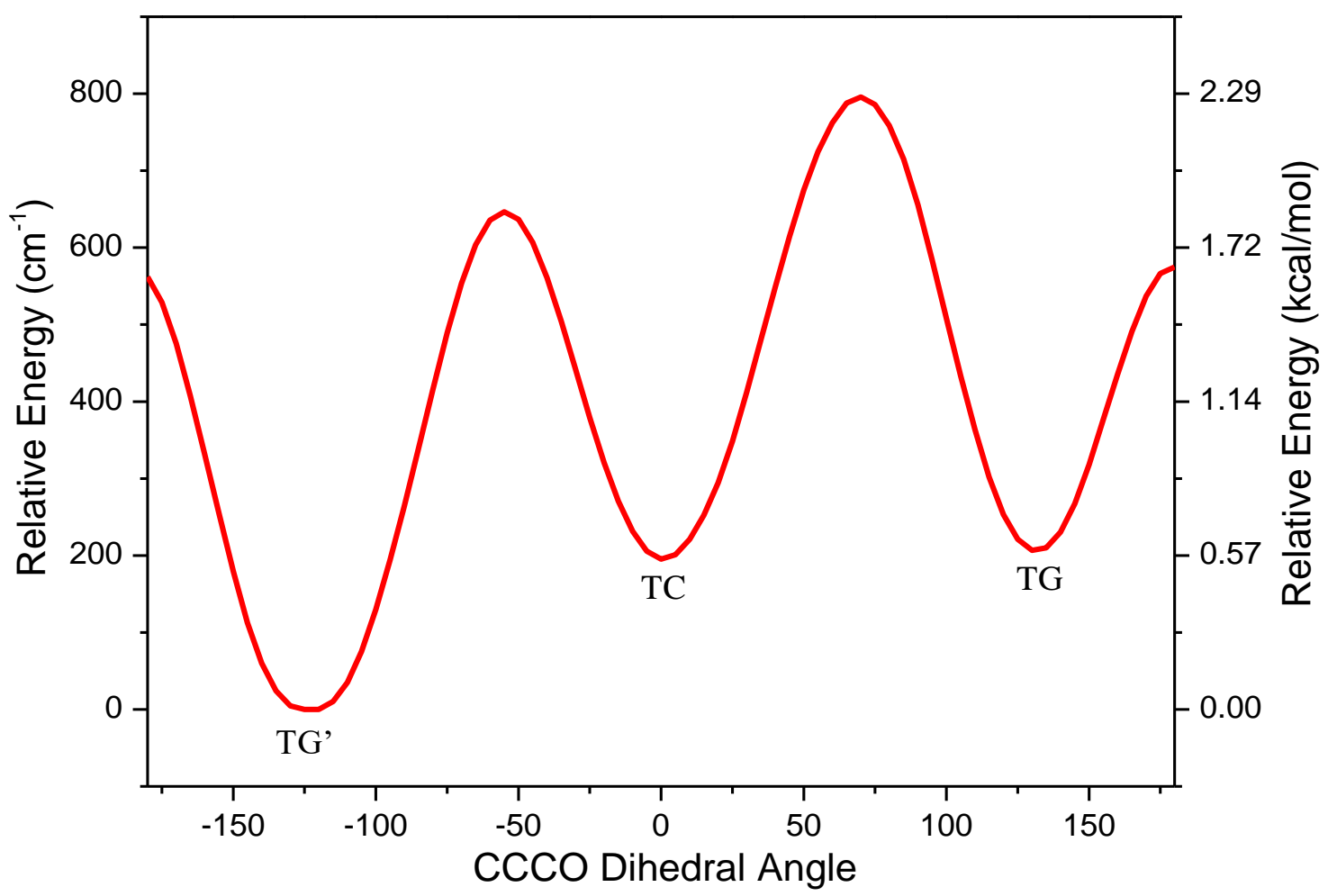

Figure 8.16. A scan of the potential energy surface of 3-MAOO along the coordinate of the CCCO dihedral angle. Molecular geometry is optimized at each step of the scan. 


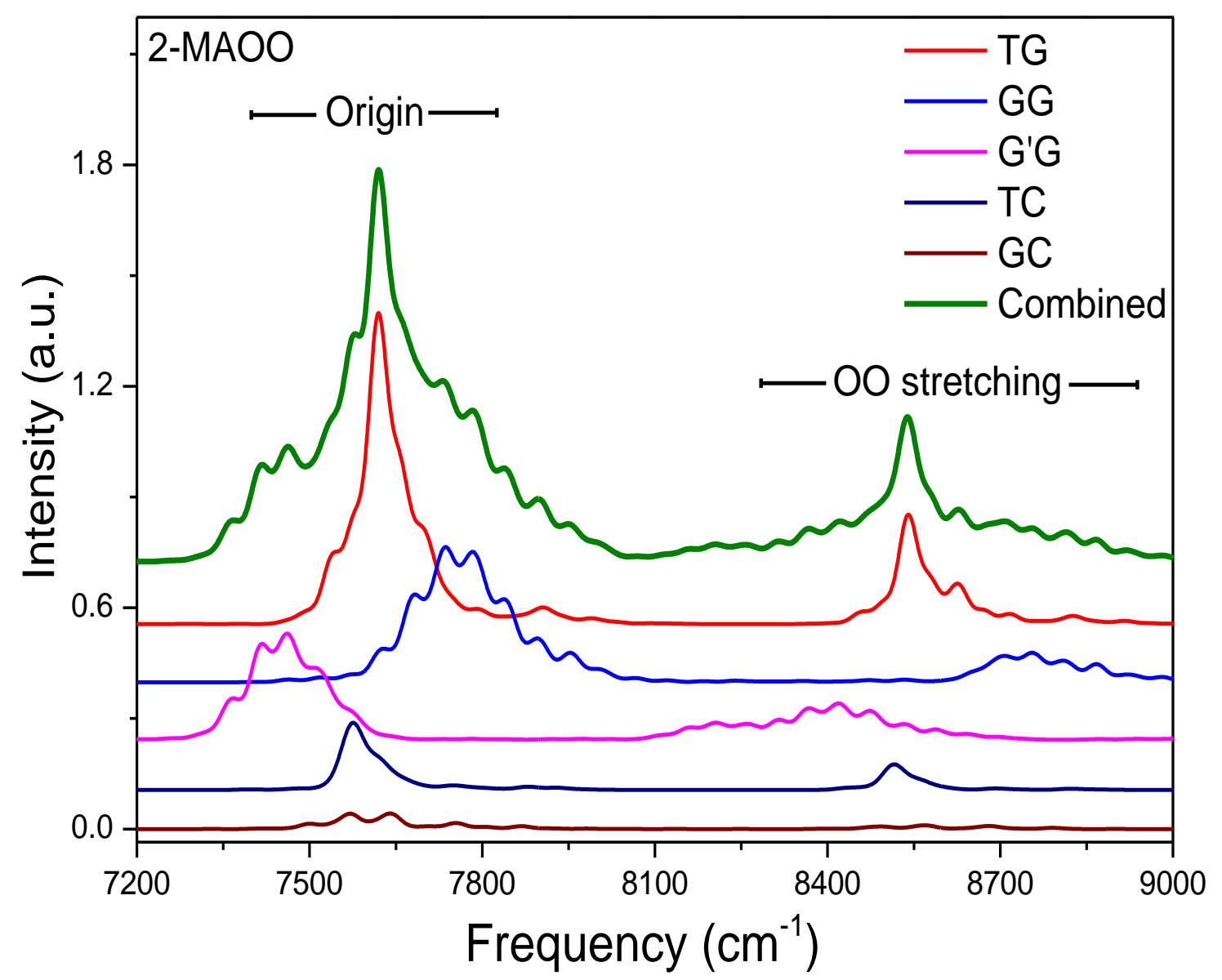

Figure 8.17. Simulated spectra of 2-MAOO conformers. Overall spectrum of 2-MAOO is calculated as summation of spectra of all conformers weighted by their relative populations at $300 \mathrm{~K}$. All spectra are offset for clarity. 


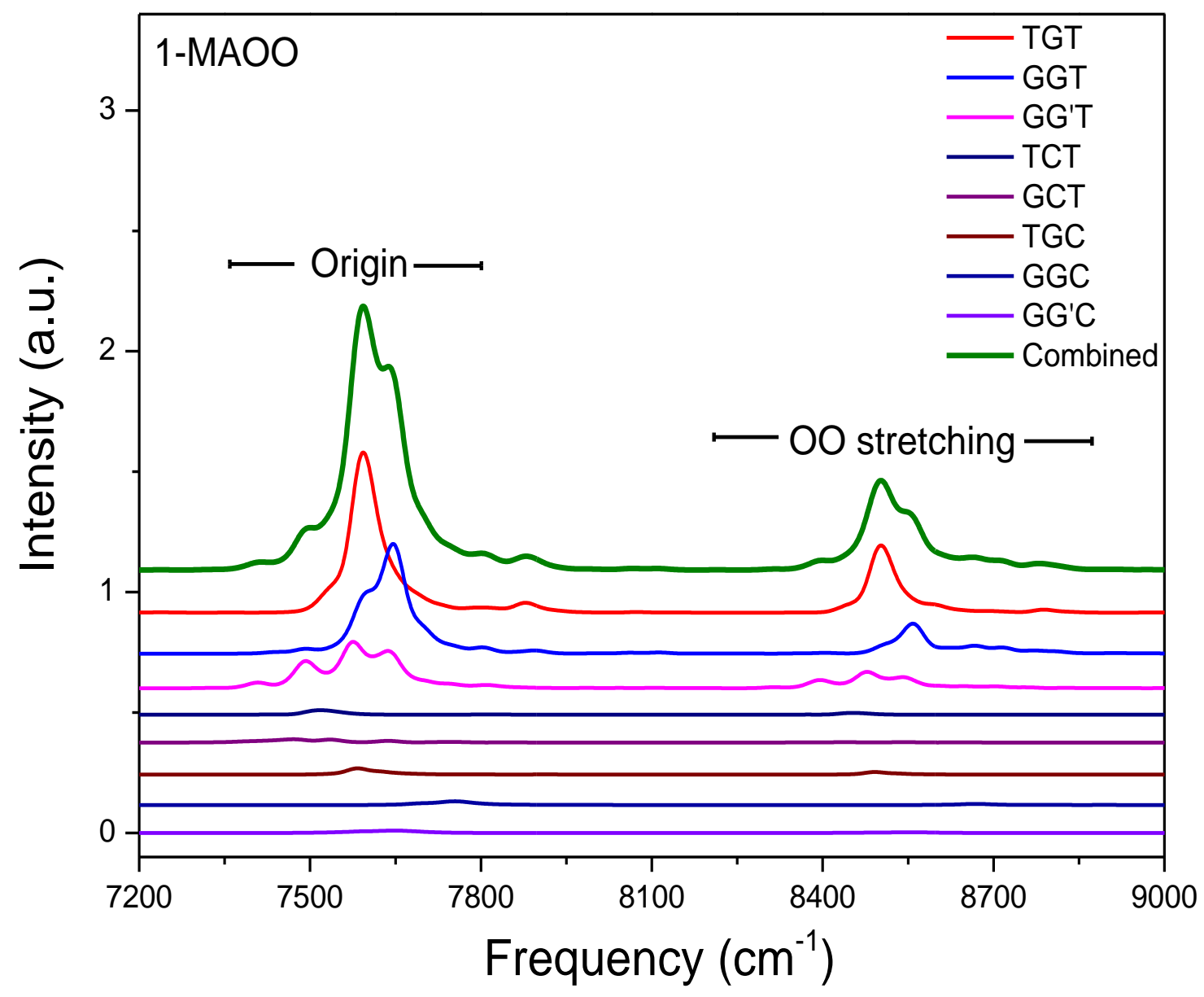

Figure 8.18. Simulated spectra of 1-MAOO conformers. Overall spectrum of 2-MAOO is calculated as summation of spectra of all conformers weighted by their relative populations at $300 \mathrm{~K}$. All spectra are offset for clarity. 


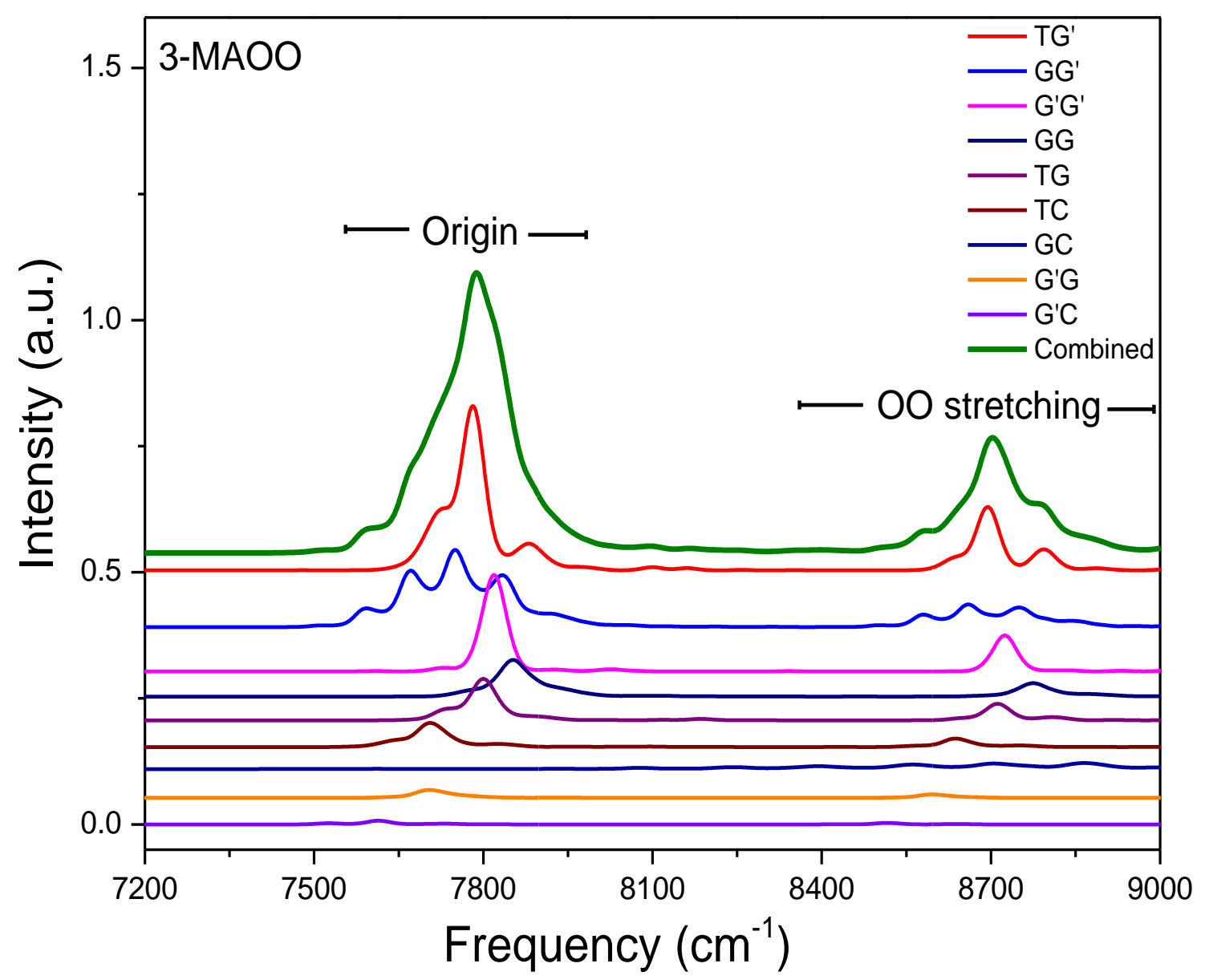

Figure 8.19. Simulated spectra of 3-MAOO conformers. Overall spectrum of 3MAOO is calculated as summation of spectra of all conformers weighted by their relative populations at $300 \mathrm{~K}$. All spectra are offset for clarity. 


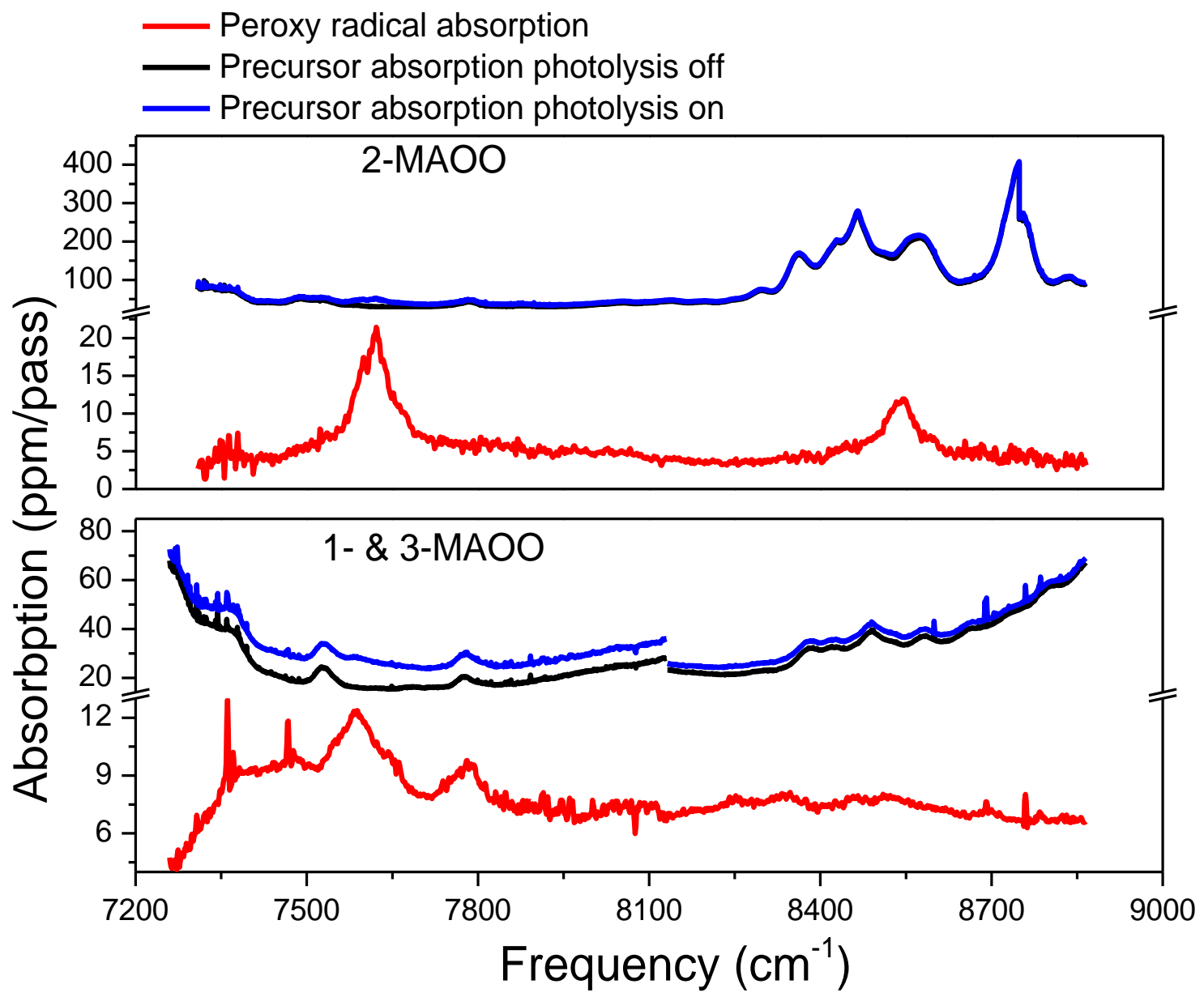

Figure 8.20. CRD spectra of MAOO isomers (red) and their precursors: 3-chloro-2methyl-1-propene for 2-MAOO production (top), and 75\% 1-chloro-2-butene and 25\% 3-chloro-1-butene mixture for 1- and 3-MAOO production (bottom). The free radical spectra are obtained by subtracting the "photolysis laser off" spectrum (black) from the "photolysis laser on" spectrum (blue). 


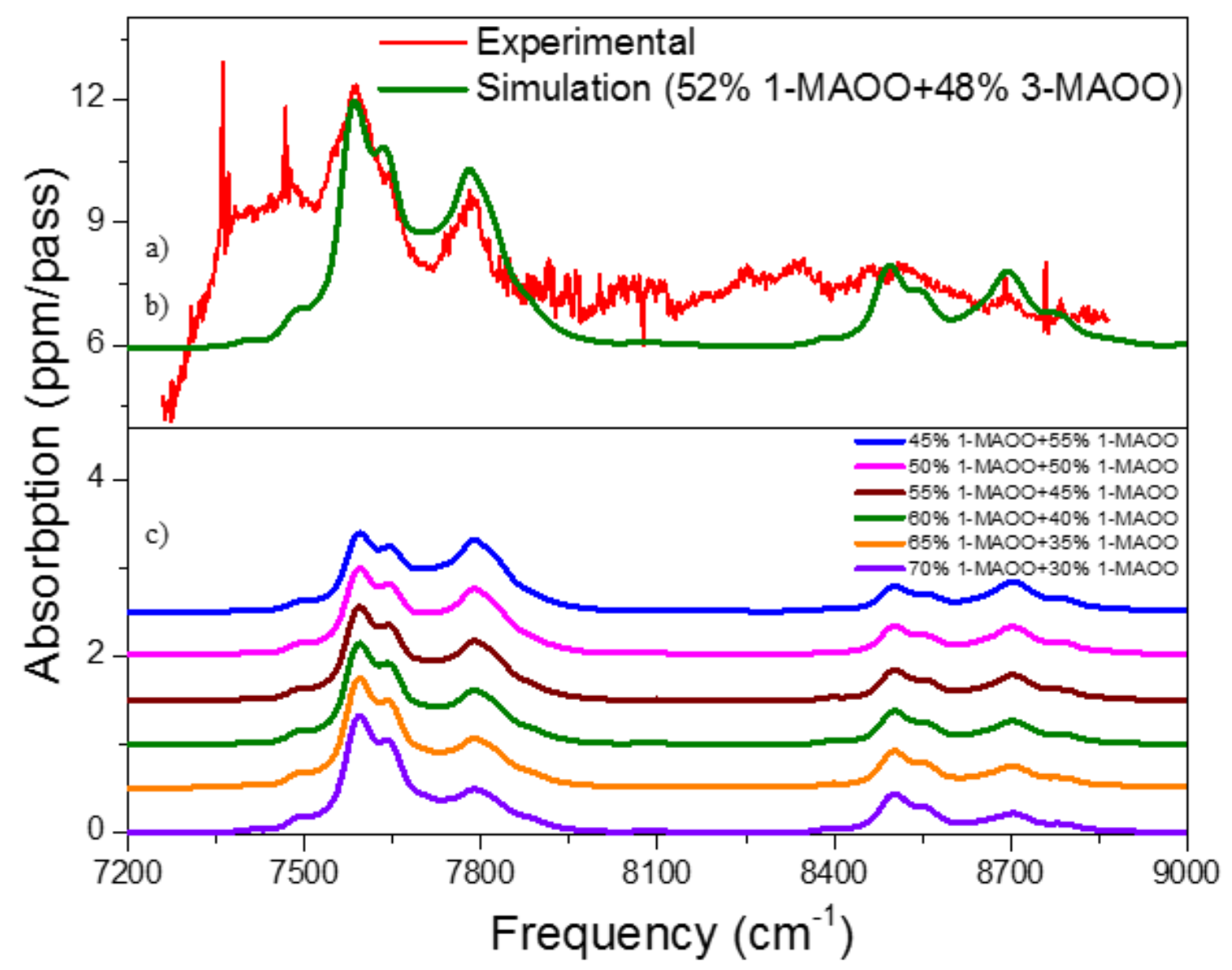

Figure 8.21. (a) CRD spectrum of the 1- MAOO and 3-MAOO radicals. (b) Simulated spectrum of 52\% 1-MAOO + 48\% 3-MAOO (c) Simulated spectra with different population ratios of 1-MAOO and 3- MAOO. Spectra are offset for clarity. 


\section{CHAPTER IX}

\section{CONCLUSIONS}

Alkoxy and peroxy radicals are important reaction intermediates in the combustion of fossil fuels and the atmosphere, especially in the troposphere. Spectroscopic study of the free radicals, namely substituted and unsaturated alkoxy and peroxy radicals can advance our understanding of many important chemical processes, including the combustion of fossil fuels and biofuels in internal combustion engines, and oxidation of hydrocarbons in the atmosphere. Spectroscopic characterization of these intermediates can provide information on their structural as well as dynamical properties. Spectroscopy of these intermediates can also help understand interesting molecular phenomena like the pseudo-Jahn Teller effect $(\mathrm{pJT})$.

LIF, DF, and CRDS techniques have been implemented to study reactive chemical intermediates. Spectra of isobutoxy, 2-methyl-1-butoxy, isopentoxy, 1-, 2-, 3-, and 4MCHO and methyl allyl peroxy radicals were obtained, analyzed and assigned on the basis of vibrational frequencies and Franck-Condon factors predicted by quantum chemical calculations. These calculations provided useful spectroscopic parameters such as rotational constants, harmonic and anharmonic vibrational frequencies, electronic excitation energies, and intensities of vibronic transitions. 
LIF spectrum of isopentoxy and DF spectra of isobutoxy, 2-methyl-1-butoxy, isopentoxy, and MCHOs radicals were obtained, and the single-conformer assignments have been confirmed by pumping different vibronic bands in their LIF spectrum. Different vibrational structures have been observed in the DF spectra when different vibronic bands in the LIF spectrum of each radical were pumped, which suggests that those vibronic bands be assigned to different conformers. In general, spectral simulation confirms lowest-energy conformers as spectral carriers. Analysis of the spectra yields the energy separation between the vibrationless levels of the $\tilde{X}$ and $\tilde{A}$ states for most of the isomers and conformers. Both LIF and DF spectra of alkoxy radicals are usually dominated by COstretch progressions because of the large difference in CO-bond length between the ground $(\tilde{X})$ and the excited ( $\tilde{B})$ electronic states, hence the large FC factors for the transitions. However, in the case of isopentoxy radicals, $\tilde{B}$-state $\beta \mathrm{C}$-C fission or predissociation of the $\tilde{B}$ state via CO-bond breaking by highly excited electronic states may cause an absence of strong transitions to the $\mathrm{CO}$-stretch levels. When non-CO stretch bands are pumped, the DF spectra are dominated by progressions of combination bands of the $\mathrm{CO}$ stretch and the pumped modes as a result of Duschinsky mixing.

Furthermore, LIF and DF spectra often contain transitions that gain intensities from vibronic interactions. For instance, in LIF spectra of branched alkoxy radicals, such as isopentoxy, we observed low-frequency vibrational progressions that are assigned to the pJT-active $\mathrm{CH}_{2} \mathrm{O}$-wagging mode. In all cases, (except 1-MCHO), the DF spectra are dominated by $\mathrm{CO}$ stretch progressions when the origin bands are pumped and by the progression of combination bands of $\mathrm{CO}$ stretch and the pumped modes when other non$\mathrm{CO}$ stretch bands are pumped. Simulated spectra using calculated vibrational frequencies 
and FCFs well reproduced the DF spectra. The observed transitions were assigned on the basis of vibrational frequencies and FCFs predicted by quantum chemical calculations. Intensities of vibronic transitions in the DF spectra are dependent on the LIF bands pumped in the experiment, which have been explained by the difference in geometry and symmetry between the lower $\tilde{X} / \tilde{A}$ states and the highly excited $\tilde{B}$ state. Differences between DF spectra obtained by pumping different vibronic bands also suggest that for these free radicals have at least two lowest-energy conformers generated by $355 \mathrm{~nm}$ photolysis under jet-cooled conditions. Intensities of vibronic transitions in the DF spectra are dependent on the LIF bands pumped in the experiment, which have been explained by the difference in geometry and symmetry between the lower $\tilde{X} / \tilde{A}$ states and the highly excited $\tilde{\mathrm{B}}$ state. Detailed spectral analysis of MCHOs enhance the understanding of vibronic interaction mechanisms such as (pJT) effect.

DF spectra of 1-MCHO are significantly different from those of the other three positional isomers. Both chair-axial and chair-equatorial conformers of 1-MCHO has strong progressions that involve both $\mathrm{CO}$ stretch and $\mathrm{C}-\mathrm{CH}_{3}$ stretch modes. Such difference is due to different positions of the methyl group on the six-membered ring and the steric interaction between the $\mathrm{CO}$ bond and the methyl group of 1-MCHO.

We produced 1-, 2- and 3-methylallyl peroxy (MAOO') radicals by $193 \mathrm{~nm}$ photolysis of methyl-substituted allyl chlorides in the presence of $\mathrm{O}_{2}$ and detected them using room-temperature CRD spectroscopy. The vibronic structure of experimentally observed spectra was simulated. The reaction intermediates for the production of 1- and 3MAOO radicals, $\mathrm{CH}_{3} \mathrm{CHCHCH}_{2}$, is a resonance-stabilized free radical. CRD spectra of 1and 3-MAOO radicals obtained using different precursors (1-chloro-2-butene and chloro- 
1-butene) suggest that allylic rearrangement between the two resonance structures $\left(\mathrm{CH}_{3} \mathrm{CH}=\mathrm{CHCH}_{2}\right.$ and $\left.\mathrm{CH}_{3} \mathrm{CH} \cdot \mathrm{CH}=\mathrm{CH}_{2}\right)$ is significantly faster than oxygen addition. The branching ratio between the terminal and non-terminal oxygen addition to forming 1 - and 3-MAOO, respectively, were predicted to be 52:48 on the basis of calculated spin densities, which agrees qualitatively with the experimental CRD spectra of the two peroxy isomers. 


\section{REFERENCES}

1. Finlayson-Pitts, B.; Pitts, J., Chemistry of the upper and lower atmosphere: theory, experiments, and applications, acad. Press: 2000.

2. Orlando, J. J.; Tyndall, G. S.; Wallington, T. J., The atmospheric chemistry of alkoxy radicals. Chemical reviews 2003, 103 (12), 4657-4690.

3. Pilling, M. J., Interactions between theory and experiment in the investigation of elementary reactions of importance in combustion. Chemical Society Reviews 2008, 37 (4), 676-685.

4. Compton, R.; Hancock, G., Chemical kinetics. Elsevier, Amsterdam: 1997.

5. Waite, J.; Young, D.; Cravens, T.; Coates, A.; Crary, F.; Magee, B.; Westlake, J., The process of tholin formation in Titan's upper atmosphere. Science 2007, 316 (5826), 870-875.

6. Atkinson, R., Atmospheric reactions of alkoxy and $\beta$-hydroxyalkoxy radicals. International Journal of Chemical Kinetics 1997, 29 (2), 99-111.

7. Jenkin, M. E.; Hayman, G. D., Photochemical ozone creation potentials for oxygenated volatile organic compounds: sensitivity to variations in kinetic and mechanistic parameters. Atmospheric environment 1999, 33 (8), 1275-1293.

8. Pilling, M., Comprehensive chemical kinetics. Elsevier Amsterdam: 1997. 
9. Curran, H. J.; Gaffuri, P.; Pitz, W. J.; Westbrook, C. K., A comprehensive modeling study of n-heptane oxidation. Combustion and flame 1998, 114 (1-2), $149-177$.

10. Ebata, T.; Yanagishita, H.; Obi, K.; Tanaka, I., $\tilde{A} \rightarrow \tilde{X}$ fluorescence spectra of $\mathrm{CH}_{3} \mathrm{O}$ and $\mathrm{C}_{2} \mathrm{H}_{5} \mathrm{O}$ generated by the ARF laser photolysis of alkyl nitrites. Chemical Physics 1982, 69 (1-2), 27-33.

11. Endo, Y.; Saito, S.; Hirota, E., The microwave spectrum of the methoxy radical $\mathrm{CH}_{3} \mathrm{O}$. The Journal of chemical physics 1984, 81 (1), 122-135.

12. Foster, S. C.; Misra, P.; Lin, T. Y. D.; Damo, C. P.; Carter, C. C.; Miller, T. A., Free jet-cooled laser-induced fluorescence spectrum of methoxy. 1. Vibronic analysis of the $\tilde{A}$ and $\tilde{X}$ states. The Journal of Physical Chemistry 1988, 92 (21), 5914-5921.

13. Inoue, G.; Akimoto, H.; Okuda, M., Laser-induced fluorescence spectra of $\mathrm{CH}_{3} \mathrm{O}$. Chemical Physics Letters 1979, 63 (2), 213-216.

14. Inoue, G.; Akimoto, H.; Okuda, M., Spectroscopy of the $\mathrm{CH}_{3} \mathrm{O} \tilde{\mathrm{A}}^{2} \mathrm{~A}_{1}-\tilde{\mathrm{X}}^{2} \mathrm{E}$ system by laser-excited fluorescence method. The Journal of Chemical Physics 1980, 72 (3), 1769-1775.

15. Liu, J.; Chen, M.-W.; Melnik, D.; Yi, J. T.; Miller, T. A., The spectroscopic characterization of the methoxy radical. I. Rotationally resolved $\tilde{\mathrm{A}}{ }^{2} \mathrm{~A}_{1^{-}} \tilde{\mathrm{X}} \mathrm{E}_{2}$ electronic spectra of $\mathrm{CH}_{3} \mathrm{O}$. The Journal of chemical physics 2009, 130 (7), 074302.

16. Liu, X.; Damo, C. P.; Lin, T.-Y. D.; Foster, S. C.; Misra, P.; Yu, L.; Miller, T. A., Free jet-cooled laser-induced fluorescence spectrum of methoxy radical. 2. 
Rotational analysis of the $\tilde{\mathrm{A}}{ }^{2} \mathrm{~A}_{1-} \tilde{\mathrm{X}} \mathrm{E}_{2}$ electronic transition. The Journal of Physical Chemistry 1989, 93 (6), 2266-2275.

17. Ohbayashi, K., H.. Akimoto, and I. Tanaka. J. Phys. Chem 1977, 81, 798.

18. Powers, D. E.; Pushkarsky, M. B.; Miller, T. A., Rovibronic analysis of the laser induced fluorescence excitation spectrum of the jet-cooled methoxy radical. The Journal of chemical physics 1997, 106 (17), 6863-6877.

19. Villenave, E.; Lesclaux, R., Kinetics of the cross reactions of $\mathrm{CH}_{3} \mathrm{O}_{2}$ and $\mathrm{C}_{2} \mathrm{H}_{5} \mathrm{O}_{2}$ radicals with selected peroxy radicals. The Journal of Physical Chemistry 1996, $100(34), 14372-14382$.

20. Wendt, H.; Hunziker, H., Electronic absorption spectrum of $\mathrm{CH}_{3} \mathrm{O}$. The Journal of Chemical Physics 1979, 71 (12), 5202-5205.

21. Choi, H.; Bise, R. T.; Neumark, D. M., Photodissociation dynamics of the ethoxy radical $\left(\mathrm{C}_{2} \mathrm{H}_{5} \mathrm{O}\right)$. The Journal of Physical Chemistry A 2000, 104 (45), 1011210118.

22. Inoue, G.; Okuda, M.; Akimoto, H., Laser-induced fluorescence of the $\mathrm{C}_{2} \mathrm{H}_{5} \mathrm{O}$ radical. The Journal of Chemical Physics 1981, 75 (5), 2060-2065.

23. Style, D.; Ward, J., Fluorescent spectra from ethyl nitrate. Part 1.- Supposed emissions from alkoxy radicals and $\mathrm{NO}_{2}$. Transactions of the Faraday Society 1953, 49, 999-1002.

24. Tan, X. Q.; Williamson, J. M.; Foster, S. C.; Miller, T. A., Rotationally resolved electronic excitation spectra of the ethoxy $\tilde{\mathrm{B}} \leftarrow \tilde{\mathrm{X}}$ transition. The Journal of Physical Chemistry 1993, 97 (37), 9311-9316. 
25. Bai, J.; Okabe, H.; Halpern, J. B., Fluorescence spectrum and lifetime of i- $\mathrm{C}_{3} \mathrm{H}_{7} \mathrm{O}$ radical. Chemical physics letters 1988, 149 (1), 37-39.

26. Chhantyal-Pun, R.; Roudjane, M.; Melnik, D. G.; Miller, T. A.; Liu, J., Jet-cooled laser-induced fluorescence spectroscopy of isopropoxy radical: vibronic analysis of $\tilde{\mathrm{B}}-\tilde{\mathrm{X}}$ and $\tilde{\mathrm{B}}-\tilde{\mathrm{A}}$ band systems. The Journal of Physical Chemistry A 2014, 118 (51), 11852-11870.

27. Ohbayashi, K.; Akimoto, H.; Tanaka, I., Emission spectra of methoxyl, ethoxyl, and isopropoxyl radicals. The Journal of Physical Chemistry 1977, 81 (8), 798-802.

28. Carter, C. C.; Gopalakrishnan, S.; Atwell, J. R.; Miller, T. A., Laser excitation spectra of large alkoxy radicals containing 5- 12 carbon atoms. The Journal of Physical Chemistry A 2001, 105 (13), 2925-2928.

29. Liang, G.; Liu, C.; Hao, H.; Zu, L.; Fang, W., Laser-induced fluorescence of isobutoxy in competition with ground state decomposition. The Journal of Physical Chemistry A 2013, 117 (50), 13229-13235.

30. Wang, C.; Deng, W.; Shemesh, L. G.; Lilien, M. D.; Katz, D. R.; Dibble, T. S., Observation of fluorescence excitation spectra of tert-pentoxy and 3-pentoxy radicals. The Journal of Physical Chemistry A 2000, 104 (45), 10368-10373.

31. Wang, C.; Shemesh, L. G.; Deng, W.; Lilien, M. D.; Dibble, T. S., Laser-induced fluorescence excitation spectra of tert-butoxy and 2-butoxy radicals. The Journal of Physical Chemistry A 1999, 103 (41), 8207-8212.

32. Juaristi, E., Conformational behavior of six-membered rings. VCH: 1995.

33. Liu, J.; Miller, T. A., Jet-cooled laser-induced fluorescence spectroscopy of cyclohexoxy: Rotational and fine structure of molecules in nearly degenerate 
electronic states. The Journal of Physical Chemistry A 2014, 118 (51), 1187111890.

34. Jin, J.; Sioutis, I.; Tarczay, G.; Gopalakrishnan, S.; Bezant, A.; Miller, T. A., Dispersed fluorescence spectroscopy of primary and secondary alkoxy radicals. The Journal of chemical physics 2004, 121 (23), 11780-11797.

35. Zu, L.; Liu, J.; Tarczay, G.; Dupré, P.; Miller, T. A., Jet-cooled laser spectroscopy of the cyclohexoxy radical. The Journal of chemical physics 2004, 120 (22), 1057910593.

36. Lin, J.; Wu, Q.; Liang, G.; Zu, L.; Fang, W., Jet-cooled laser-induced fluorescence spectroscopy of methylcyclohexoxy radicals. RSC Advances 2012, 2 (2), 583-589.

37. Wu, Q.; Liang, G.; Zu, L.; Fang, W., Vibrationally resolved LIF spectrum of tertiary methylcyclohexoxy radical. The Journal of Physical Chemistry A 2012, 116 (12), 3156-3162.

38. Rienstra-Kiracofe, J. C.; Allen, W. D.; Schaefer, H. F., The $\mathrm{C}_{2} \mathrm{H}_{5}+\mathrm{O}_{2}$ reaction mechanism: high-level ab initio characterizations. The Journal of Physical Chemistry A 2000, 104 (44), 9823-9840.

39. Orlando, J. J.; Tyndall, G. S., Laboratory studies of organic peroxy radical chemistry: an overview with emphasis on recent issues of atmospheric significance. Chemical Society Reviews 2012, 41 (19), 6294-6317.

40. Zádor, J.; Taatjes, C. A.; Fernandes, R. X., Kinetics of elementary reactions in lowtemperature autoignition chemistry. Progress in energy and combustion science 2011, 37 (4), 371-421.

41. Glassman, I., Combustion, Academic Press Orlando FL. 1987. 
42. Wang, S.; Miller, D. L.; Cernansky, N. P.; Curran, H. J.; Pitz, W. J.; Westbrook, C. K., A flow reactor study of neopentane oxidation at 8 atmospheres: experiments and modeling. Combustion and Flame 1999, 118 (3), 415-430.

43. Balaban, A.; Motoc, I., Chemical graphs. XXXVI, correlations between octane numbers and topological indices of alkanes. Math. Chem.(MATCH) 1979, 5, 197218.

44. Gaffuri, P.; Faravelli, T.; Ranzi, E.; Cernansky, N. P.; Miller, D.; d'Anna, A.; Ciajolo, A., Comprehensive kinetic model for the low temperature oxidation of hydrocarbons. AIChE journal 1997, 43 (5), 1278-1286.

45. Morley, C., A fundamentally based correlation between alkane structure and octane number. Combustion science and technology 1987, 55 (4-6), 115-123.

46. Perdih, A.; Perdih, F., Chemical interpretation of octane number. Acta Chimica Slovenica 2006, 53 (3).

47. Warnatz, J.; Mass, U.; Dibble, R., Combustion: physical and chemical fundamentals, modeling and simulation, experiments, pollutant formation, 1999. Springer-Verlag, Berlin.

48. Westbrook, C., Chemistry behind engine knock. Chemistry and Industry 1992, 5626.

49. Jenkin, M. E.; Clemitshaw, K. C., Ozone and other secondary photochemical pollutants: chemical processes governing their formation in the planetary boundary layer. Atmospheric Environment 2000, 34 (16), 2499-2527.

50. Lightfoot, P. D.; Cox, R.; Crowley, J.; Destriau, M.; Hayman, G.; Jenkin, M.; Moortgat, G.; Zabel, F., Organic peroxy radicals: kinetics, spectroscopy and 
tropospheric chemistry. Atmospheric Environment. Part A. General Topics 1992, $26(10), 1805-1961$.

51. Tyndall, G.; Cox, R.; Granier, C.; Lesclaux, R.; Moortgat, G.; Pilling, M.; Ravishankara, A.; Wallington, T., Atmospheric chemistry of small organic peroxy radicals. Journal of Geophysical Research: Atmospheres 2001, 106 (D11), 1215712182.

52. Guenther, A.; Hewitt, C. N.; Erickson, D.; Fall, R.; Geron, C.; Graedel, T.; Harley, P.; Klinger, L.; Lerdau, M.; McKay, W., A global model of natural volatile organic compound emissions. Journal of Geophysical Research: Atmospheres 1995, 100 (D5), 8873-8892.

53. Ravishankara, A., Are chlorine atoms significant tropospheric free radicals? Proceedings of the National Academy of Sciences 2009, 106 (33), 13639-13640.

54. Sawada, S.; Totsuka, T., Natural and anthropogenic sources and fate of atmospheric ethylene. Atmospheric Environment (1967) 1986, 20 (5), 821-832.

55. Alfassi, Z. B., The chemistry of free radicals: peroxyl radicals. Wiley: 1997; Vol. 2.

56. Chameides, W.; Lindsay, R.; Richardson, J.; Kiang, C., The role of biogenic hydrocarbons in urban photochemical smog: Atlanta as a case study. Science 1988, 241 (4872), 1473-1475.

57. Rasmussen, R.; Khalil, M., Isoprene over the Amazon basin. Journal of Geophysical Research: Atmospheres 1988, 93 (D2), 1417-1421. 
58. Trainer, M.; Williams, E. J.; Parrish, D.; Buhr, M.; Allwine, E.; Westberg, H.; Fehsenfeld, F. C.; Liu, S. C., Models and observations of the impact of natural hydrocarbons on rural ozone. Nature 1987, 329 (6141), 705.

59. Calvert, J. G., Atkinson, R., Kerr, J. A., Madronich, S., Moortgat, G., Wallington, T. J., \& Yarwood, G., The Mechanisms of Atmospheric Oxidation of the Alkenes. Oxford University Press, New York, New York: 2000.

60. Dibble, T. S., Computations on the $\tilde{\mathrm{A}}-\tilde{\mathrm{X}}$ transition of isoprene-OH$-\mathrm{O}_{2}$ peroxy radicals. Journal of computational chemistry 2005, 26 (8), 836-845.

61. Ghosh, B.; Bugarin, A.; Connell, B. T.; North, S. W., Isomer-selective study of the $\mathrm{OH}$-initiated oxidation of isoprene in the presence of $\mathrm{O}_{2}$ and $\mathrm{NO}_{2}$. The major $\mathrm{OH}$ addition channel. The Journal of Physical Chemistry A 2010, 114 (7), 25532560.

62. Lei, W.; Zhang, R., Theoretical study of hydroxyisoprene alkoxy radicals and their decomposition pathways. The Journal of Physical Chemistry A 2001, 105 (15), 3808-3815.

63. Lei, W.; Zhang, R.; McGivern, W. S.; Derecskei-Kovacs, A.; North, S. W., Theoretical study of $\mathrm{OH}-\mathrm{O}_{2}-$ isoprene peroxy radicals. The Journal of Physical Chemistry A 2001, 105 (2), 471-477.

64. Surratt, J. D.; Chan, A. W.; Eddingsaas, N. C.; Chan, M.; Loza, C. L.; Kwan, A. J.; Hersey, S. P.; Flagan, R. C.; Wennberg, P. O.; Seinfeld, J. H., Reactive intermediates revealed in secondary organic aerosol formation from isoprene. Proceedings of the National Academy of Sciences 2010, 107 (15), 6640-6645. 
65. Zhang, R.; Lei, W., Reactions of $\mathrm{O}_{2}+$ with $\mathrm{OH}$-isoprene adduct isomers: Exothermicity, product ions, and rate constants. The Journal of Chemical Physics 2000, $113(19), 8574-8579$.

66. Boyd, S. L.; Boyd, R. J.; Shi, Z.; Barclay, L. R. C.; Porter, N. A., A theoretical investigation of the 1,3-migration in allylperoxyl radicals. Journal of the American Chemical Society 1993, 115 (2), 687-693.

67. Brill, W. F., The isolation and rearrangement of pure acyclic allylic hydroperoxides. Journal of the American Chemical Society 1965, 87 (14), 32863287.

68. Porter, N. A.; Caldwell, S. E.; Mills, K. A., Mechanisms of free radical oxidation of unsaturated lipids. Lipids 1995, 30 (4), 277-290.

69. Schenck, G. O.; Neumüller, O. A.; Eisfeld, W., Zur photosensibilisierten Autoxydation der Steroide: $\Delta 5$-Steroid-7 $\alpha$-hydroperoxyde und-7-ketone durch Allylumlagerung von $\Delta 6$-Steroid-5a-hydroperoxyden. Justus Liebigs Annalen der Chemie 1958, 618 (1), 202-210.

70. Jenkin, M. E.; Boyd, A. A.; Lesclaux, R., Peroxy radical kinetics resulting from the OH-initiated oxidation of 1, 3-butadiene, 2, 3-dimethyl-1, 3-butadiene and isoprene. Journal of Atmospheric Chemistry 1998, 29 (3), 267-298.

71. Jenkin, M. E.; Murrells, T. P.; Shalliker, S. J.; Hayman, G. D., Kinetics and product study of the self-reactions of allyl and allyl peroxy radicals at $296 \mathrm{~K}$. Journal of the Chemical Society, Faraday Transactions 1993, 89 (3), 433-446. 
72. Thomas, P. S.; Miller, T. A., Cavity Ringdown Spectroscopy of the NIR $\tilde{A}-\tilde{X}$ Electronic Transition of Allyl Peroxy Radical $\left(\mathrm{H}_{2} \mathrm{CCH}-\mathrm{CH}_{2} \mathrm{OO} \cdot\right)$. Chemical Physics Letters 2010, 491 (4), 123-131.

73. Martin, R. S.; Westberg, H.; Allwine, E.; Ashman, L.; Farmer, J. C.; Lamb, B., Measurement of isoprene and its atmospheric oxidation products in a central Pennsylvania deciduous forest. Journal of Atmospheric Chemistry 1991, 13 (1), 132.

74. Wiedinmyer, C.; Friedfeld, S.; Baugh, W.; Greenberg, J.; Guenther, A.; Fraser, M.; Allen, D., Measurement and analysis of atmospheric concentrations of isoprene and its reaction products in central Texas. Atmospheric Environment 2001, 35 (6), 1001-1013.

75. Brown, S.; Degouw, J.; Warneke, C.; Ryerson, T.; Dubé, W.; Atlas, E.; Weber, R.; Peltier, R.; Neuman, J.; Roberts, J., Nocturnal isoprene oxidation over the Northeast United States in summer and its impact on reactive nitrogen partitioning and secondary organic aerosol. Atmospheric Chemistry and Physics 2009, 9 (9), 3027-3042.

76. Campuzano-Jost, P.; Williams, M.; D'Otton, L.; Hynes, A. J., Kinetics and mechanism of the reaction of the hydroxyl radical with $\mathrm{h} 8$-isoprene and $\mathrm{d} 8$ isoprene: isoprene absorption cross sections, rate coefficients, and the mechanism of hydroperoxyl radical production. The Journal of Physical Chemistry A 2004, 108 (9), 1537-1551.

77. Greenwald, E. E.; Ghosh, B.; Anderson, K. C.; Dooley, K. S.; Zou, P.; Selby, T.; Osborn, D. L.; Meloni, G.; Taatjes, C. A.; Goulay, F., Isomer-selective study 
of the $\mathrm{OH}$ initiated oxidation of isoprene in the presence of $\mathrm{O}_{2}$ and NO. I. The minor inner OH-addition channel. The Journal of Physical Chemistry A 2009, 114 (2), 904-912.

78. Lee, W.; Baasandorj, M.; Stevens, P. S.; Hites, R. A., Monitoring OH-initiated oxidation kinetics of isoprene and its products using online mass spectrometry. Environmental science \& technology 2005, 39 (4), 1030-1036.

79. Murrells, T. P.; Jenkin, M. E.; Shalliker, S. J.; Hayman, G. D., Lasser flash photolysis study of the UV spectrum and kinetics of reactions of $\mathrm{HOCH}_{2} \mathrm{CH}_{2} \mathrm{O}_{2}$ radicals. Journal of the Chemical Society, Faraday Transactions 1991, 87 (15), 2351-2360.

80. Paulot, F.; Crounse, J. D.; Kjaergaard, H. G.; Kürten, A.; Clair, J. M. S.; Seinfeld, J. H.; Wennberg, P. O., Unexpected epoxide formation in the gas-phase photooxidation of isoprene. Science 2009, 325 (5941), 730-733.

81. Peeters, J.; Nguyen, T. L.; Vereecken, L., $\mathrm{HO}_{\mathrm{x}}$ radical regeneration in the oxidation of isoprene. Physical Chemistry Chemical Physics 2009, 11 (28), 5935-5939.

82. Jafri, J. A.; Phillips, D. H., Ground and lower excited states of methylperoxy radical, $\mathrm{CH}_{3} \mathrm{O}_{2}$ : a computational investigation. Journal of the American Chemical Society 1990, $112(7), 2586-2590$.

83. Newman, S. M.; Lane, I. C.; Orr-Ewing, A. J.; Newnham, D. A.; Ballard, J., Integrated absorption intensity and Einstein coefficients for the $\mathrm{O}_{2} \mathrm{a}^{1} \Delta_{\mathrm{g}}-\mathrm{X}^{3} \Sigma_{\mathrm{g}}{ }^{-}(0$, 0) transition: A comparison of cavity ringdown and high resolution Fourier transform spectroscopy with a long-path absorption cell. The Journal of chemical physics 1999, 110 (22), 10749-10757. 
84. Carter, C. C.; Atwell, J. R.; Gopalakrishnan, S.; Miller, T. A., Jet-cooled laserinduced fluorescence spectroscopy of some alkoxy radicals. The Journal of Physical Chemistry A 2000, 104 (40), 9165-9170.

85. Liu, J.; Chen, M.-W.; Melnik, D.; Miller, T. A.; Endo, Y.; Hirota, E., The spectroscopic characterization of the methoxy radical. II. Rotationally resolved $\tilde{A}$ ${ }^{2} \mathrm{~A}_{1}-\tilde{\mathrm{X}} \mathrm{E}_{2}$ electronic and $\tilde{\mathrm{X}} \mathrm{E}_{2}$ microwave spectra of the perdeuteromethoxy radical $\mathrm{CD}_{3} \mathrm{O}$. The Journal of chemical physics 2009, 130 (7), 074303.

86. Brock, L.; Rohlfing, E. A., Spectroscopic studies of the $\tilde{B}^{2} \mathrm{~A}^{\prime \prime}-\tilde{\mathrm{X}}^{2} \mathrm{~A}^{\prime \prime}$ system of the jet-cooled vinoxy radical. The Journal of chemical physics 1997, 106 (24), 10048-10065.

87. Heaven, M.; Dimauro, L.; Miller, T. A., Laser-induced fluorescence spectra of free-jet cooled organic free radicals. Vinoxy, cyclopentadienyl, and benzyl. Chemical Physics Letters 1983, 95 (4-5), 347-351.

88. Gopalakrishnan, S.; Zu, L.; Miller, T. A., Rotationally resolved electronic spectra of the $\tilde{B}-\tilde{X}$ transition in multiple conformers of 1-butoxy and 1-pentoxy radicals. The Journal of Physical Chemistry A 2003, 107 (26), 5189-5201.

89. Miller, T. A., Spectroscopic probing and diagnostics of the geometric structure of the alkoxy and alkyl peroxy radical intermediates. Molecular Physics 2006, 104 (16-17), 2581-2593.

90. Reza, M. A.; Paul, A. C.; Reilly, N. J.; Liu, J., Laser-induced fluorescence and dispersed fluorescence spectroscopy of jet-cooled isopentoxy radicals. The Journal of Physical Chemistry A 2019, 123 (39), 8441-8447. 
91. Paul, A. C.; Reza, M. A.; Shuvra, P. D.; Liu, J. In Laser-induced fluorescence and dispersed-fluorescence spectroscopy of jet-cooled calcium monoalkoxide radicals, 73rd International Symposium on Molecular Spectroscopy, 2018.

92. Paul, A. C.; Sharma, K.; Reza, M. A.; Telfah, H.; Miller, T. A.; Liu, J., Laserinduced fluorescence and dispersed-fluorescence spectroscopy of the $\tilde{\mathrm{A}}^{2} \mathrm{E}-\tilde{\mathrm{X}}^{2} \mathrm{~A}_{1}$ transition of jet-cooled calcium methoxide $\left(\mathrm{CaOCH}_{3}\right)$ radicals. 2019, 151 (13), 134303.

93. Alam, J.; Reza, M. A.; Mason, A.; Reilly, N. J.; Liu, J., Dispersed fluorescence spectroscopy of jet-cooled 2-, 3-, and 4-methylcyclohexoxy radicals. The Journal of Physical Chemistry A 2015, 119 (24), 6257-6268.

94. Ghigo, G.; Maranzana, A.; Tonachini, G., Combustion and atmospheric oxidation of hydrocarbons: Theoretical study of the methyl peroxyl self-reaction. The Journal of chemical physics 2003, 118 (23), 10575-10583.

95. Paul, A. C.; Reza, M. A.; Liu, J., Dispersed-fluorescence spectroscopy of jetcooled calcium ethoxide radical $\left(\mathrm{CaOC}_{2} \mathrm{H}_{5}\right)$. Journal of Molecular Spectroscopy 2016, 330, 142-146.

96. Reza, M. A.; Paul, A. C.; Reilly, N. J.; Alam, J.; Liu, J., Dispersed fluorescence spectroscopy of jet-cooled isobutoxy and 2-methyl-1-butoxy radicals. The Journal of Physical Chemistry A 2016, 120 (34), 6761-6767.

97. Alam, J.; Reza, M. A.; Mason, A.; Liu, J. In Dispersed fluorescence spectroscopy of jet-cooled methylcyclohexoxy radicals, 70th International Symposium on Molecular Spectroscopy, 2015. 
98. Paul, A. C.; Reza, M. A.; Liu, J. In Dispersed-fluorescence spectroscopy of jetcooled calcium ethoxide radical $\left(\mathrm{CaOC}_{2} \mathrm{H}_{5}\right)$, 71st International Symposium on Molecular Spectroscopy, 2016.

99. Reza, M. A.; Reilly, N. J.; Alam, J.; Mason, A.; Liu, J. In Dispersed fluorescence spectroscopy of jet-cooled isobutoxy, 2-methyl-1-butoxy, and isopentoxy radicals, 70th International Symposium on Molecular Spectroscopy, 2015.

100. Blatt, A., Organic Synthesis II. Wiley, New York: 1963.

101. Nielsen, O. J.; Wallington, T. J., Peroxyl radicals. John Wiley and Sons, New York, NY, 1997.

102. Sharp, E. N.; Rupper, P.; Miller, T. A., The structure and spectra of organic peroxy radicals. Physical Chemistry Chemical Physics 2008, 10 (27), 3955-3981.

103. Just, G. M.; Rupper, P.; Miller, T. A.; Meerts, W. L., High-resolution cavity ringdown spectroscopy of the jet-cooled ethyl peroxy radical $\mathrm{C}_{2} \mathrm{H}_{5} \mathrm{O}_{2}$. The Journal of chemical physics 2009, 131 (18), 184303.

104. Wu, S.; Dupré, P.; Rupper, P.; Miller, T. A., The vibrationless $\tilde{\mathrm{A}} \leftarrow \tilde{\mathrm{X}}$ transition of the jet-cooled deuterated methyl peroxy radical $\mathrm{CD}_{3} \mathrm{O}_{2}$ by cavity ringdown spectroscopy. The Journal of chemical physics 2007, 127 (22), 224305.

105. Reza, M. A.; Telfah, H.; Xu, R.; Liu, J., Room-temperature cavity ring-down spectroscopy of methylallyl peroxy radicals. The Journal of Physical Chemistry A 2019, 123 (16), 3510-3517.

106. Telfah, H.; Reza, M. A.; Alam, J.; Paul, A. C.; Liu, J., Direct observation of tetrahydrofuranyl and tetrahydropyranyl peroxy radicals via cavity ring-down spectroscopy. The Journal of Physical Chemistry Letters 2018, 4475-4480. 
107. Reza, M. A.; Telfah, H.; Paul, A. C.; Alam, J.; Liu, J. In Cavity ring-down spectroscopy of 1-, 2-and 3-methyl allyl peroxy radicals, 73rd International Symposium on Molecular Spectroscopy, 2018.

108. Telfah, H.; Reza, M. A.; Paul, A. C.; Liu, J. In Observation of the $\tilde{A} \leftarrow \tilde{X}$ electronic transitions of tetrahydropyranyl and tetrahydrofuranyl peroxy radicals by room-temperature cavity ring-down spectroscopy, 73rd International Symposium on Molecular Spectroscopy, 2018.

109. Owyoung, A., High-resolution cw stimulated Raman spectroscopy in molecular hydrogen. Optics letters 1978, 2 (4), 91-93.

110. Sußmann, R.; Weber, T.; Riedle, E.; Neusser, H., Frequency shifting of pulsed narrow-band laser light in a multipass Raman cell. Optics communications 1992, 88 (4-6), 408-414.

111. Becke, A., Phys. Re V. A 1988, 38, 3098.(b) Becke. J. Chem. Phys 1993, 98 (1372), 5648.

112. Yanai, T.; Tew, D. P.; Handy, N. C., A new hybrid exchange-correlation functional using the Coulomb-attenuating method (CAM-B3LYP). Chemical Physics Letters 2004, 393 (1-3), 51-57.

113. Foresman, J. B.; Head-Gordon, M.; Pople, J. A.; Frisch, M. J., Toward a systematic molecular orbital theory for excited states. The Journal of Physical Chemistry 1992, $96(1), 135-149$.

114. Frisch, M. J.; Trucks, G. W.; Schlegel, H. B.; Scuseria, G. E.; Robb, M. A.; Cheeseman, J. R.; Scalmani, G.; Barone, V.; Petersson, G. A.; Nakatsuji, H.; Li, X.; Caricato, M.; Marenich, A.; Bloino, J.; Janesko, B. G.; Gomperts, R.; 
Mennucci, B.; Hratchian, H. P.; Ortiz, J. V.; Izmaylov, A. F.; Sonnenberg, J. L.; Williams-Young, D.; Ding, F.; Lipparini, F.; Egidi, F.; Goings, J.; Peng, B.; Petrone, A.; Henderson, T.; Ranasinghe, D.; Zakrzewski, V. G.; Gao, J.; Rega, N.; Zheng, G.; Liang, W.; Hada, M.; Ehara, M.; Toyota, K.; Fukuda, R.; Hasegawa, J.; Ishida, M.; Nakajima, T.; Honda, Y.; Kitao, O.; Nakai, H.; Vreven, T.; Throssell, K.; Montgomery Jr., J. A.; Peralta, J. E.; Ogliaro, F.; Bearpark, M.; Heyd, J. J.; Brothers, E.; Kudin, K. N.; Staroverov, V. N.; Keith, T.; Kobayashi, R.; Normand, J.; Raghavachari, K.; Rendell, A.; Burant, J. C.; Iyengar, S. S.; Tomasi, J.; Cossi, M.; Millam, J. M.; Klene, M.; Adamo, C.; Cammi, R.; Ochterski, J. W.; Martin, R. L.; Morokuma, K.; Farkas, O.; Foresman, J. B.; J., F. D. Gaussian 09 Rev. C.01, Wallingford, CT, 2016.

115. Bernath, P. F., Spectra of atoms and molecules. Oxford university press: 2015.

116. Lessinger, L., Morse oscillators, Birge-Sponer extrapolation, and the electronic absorption spectrum of I2. Journal of chemical education 1994, 71 (5), 388.

117. Wilson, E. B.; Decius, J. C.; Cross, P. C., Molecular vibrations: the theory of infrared and Raman vibrational spectra. Courier Corporation: 1980.

118. Condon, E., A theory of intensity distribution in band systems. Physical Review 1926, $28(6), 1182$.

119. Franck, J.; Dymond, E., Elementary processes of photochemical reactions. Transactions of the Faraday Society 1926, 21 (February), 536-542.

120. https://en.wikipedia.org/wiki/Franck\%E2\%80\%93Condon_principle.

121. Mozhayskiy, V. A.; Krylov, A. I. ezSpectrum (http://iopenshell.usc.edu/downloads). 
122. Duschinsky, F., The importance of the electron spectrum in multi atomic molecules. Concerning the Franck-Condon principle. Acta Physicochim. URSS 1937, 7, 551566.

123. Müller, C. W.; Newby, J. J.; Liu, C.-P.; Rodrigo, C. P.; Zwier, T. S., Duschinsky mixing between four non-totally symmetric normal coordinates in the $\mathrm{S}_{1}-\mathrm{S}_{0}$ vibronic structure of (E)-phenylvinylacetylene: a quantitative analysis. Physical Chemistry Chemical Physics 2010, 12 (10), 2331-2343.

124. Kwok, E. S.; Arey, J.; Atkinson, R., Alkoxy radical isomerization in the $\mathrm{OH}$ radical-initiated reactions of $\mathrm{C}_{4}-\mathrm{C}_{8} \mathrm{n}$-alkanes. The Journal of Physical Chemistry 1996, 100 (1), 214-219.

125. Méreau, R.; Rayez, M.-T.; Caralp, F.; Rayez, J.-C., Isomerisation reactions of alkoxy radicals: theoretical study and structure-activity relationships. Physical Chemistry Chemical Physics 2003, 5 (21), 4828-4833.

126. Vereecken, L.; Peeters, J., Decomposition of substituted alkoxy radicals—part I: a generalized structure-activity relationship for reaction barrier heights. Physical Chemistry Chemical Physics 2009, 11 (40), 9062-9074.

127. Vereecken, L.; Peeters, J., A structure-activity relationship for the rate coefficient of H-migration in substituted alkoxy radicals. Physical Chemistry Chemical Physics 2010, 12 (39), 12608-12620.

128. Frisch, M.; Trucks, G.; Schlegel, H.; Scuseria, G.; Robb, M.; Cheeseman, J.; Scalmani, G.; Barone, V.; Mennucci, B.; Petersson, G., Gaussian 09, revision A. 01. Gaussian Inc., Wallingford, CT. J. Chem. Phys: 2009. 
129. Morgan, C. A.; Pilling, M. J.; Tulloch, J. M.; Ruiz, R. P.; Bayes, K. D., Direct determination of the equilibrium constant and thermodynamic parameters for the reaction. $\mathrm{C}_{3} \mathrm{H}_{5}+\mathrm{O}_{2} \rightleftharpoons \mathrm{C}_{3} \mathrm{H}_{5} \mathrm{O}_{2}$. Journal of the Chemical Society, Faraday Transactions 2: Molecular and Chemical Physics 1982, 78 (8), 1323-1330.

130. Liu, J.; Reilly, N. J.; Mason, A.; Miller, T. A., Laser-induced fluorescence spectroscopy of jet-cooled t-butoxy. The Journal of Physical Chemistry A 2015, 119 (49), 11804-11812.

131. Chhantyal-Pun, R.; Chen, M.-W.; Miller, T. A., Laser induced fluorescence study of the $\tilde{\mathrm{B}}-\tilde{\mathrm{X}}$ transition of $\mathrm{FCH}_{2} \mathrm{CH}_{2} \mathrm{O}$. Chemical Physics Letters 2013, 555, 64-71.

132. Liu, J.; Melnik, D.; Miller, T. A., Rotationally resolved $\tilde{B} \leftarrow \tilde{X}$ electronic spectra of the isopropoxy radical: A comparative study. The Journal of chemical physics 2013, $139(9), 094308$.

133. Osborn, D. L., Reaction mechanisms on multiwell potential energy surfaces in combustion (and atmospheric) chemistry. Annual Review of Physical Chemistry 2017, 68 (1), 233-260.

134. Zu, L.; Liu, J.; Gopalakrishnan, S.; Miller, T. A., The rotationally resolved electronic spectra of several conformers of 1-hexoxy and 1-heptoxy. Canadian journal of chemistry 2004, 82 (6), 854-866.

135. Gopalakrishnan, S.; Zu, L.; Miller, T. A., Radiative and non-radiative decay of selected vibronic levels of the $\tilde{B}$ state of alkoxy radicals. Chemical physics letters 2003, 380 (5-6), 749-757. 
136. Chai, J.-D.; Head-Gordon, M., Long-range corrected hybrid density functionals with damped atom-atom dispersion corrections. Physical Chemistry Chemical Physics 2008, 10 (44), 6615-6620.

137. Malbon, C. L.; Yarkony, D. R.; Zhu, X., On the electronic structure of the ground state of cyclopentoxy. The case for a two coupled state description. Journal of Molecular Spectroscopy 2015, 311, 36-41.

138. Gopalakrishnan, S.; Carter, C. C.; Zu, L.; Stakhursky, V.; Tarczay, G.; Miller, T. A., Rotationally resolved $\tilde{\mathrm{B}}-\tilde{\mathrm{X}}$ electronic spectra of both conformers of the 1propoxy radical. The Journal of chemical physics 2003, 118 (11), 4954-4969.

139. Dillon, J. J.; Yarkony, D. R., The photoelectron spectrum of the isopropoxide anion: Nonadiabatic effects due to conical intersections and the spin-orbit interaction. The Journal of chemical physics 2009, 130 (15), 154312.

140. Dillon, J. J.; Yarkony, D. R., The photoelectron spectrum of the ethoxide anion: Conical intersections, the spin-orbit interaction, and sequence bands. The Journal of chemical physics 2009, 131 (13), 134303.

141. Stakhursky, V. L.; Zu, L.; Liu, J.; Miller, T. A., High resolution spectra and conformational analysis of 2-butoxy radical. The Journal of chemical physics 2006, 125 (9), 094316.

142. Reilly, N. J.; Cheng, L.; Stanton, J. F.; Miller, T. A.; Liu, J. In Jet-cooled laserinduced fluorescence spectroscopy of t-butoxy, 70th International Symposium on Molecular Spectroscopy, 2015. 
143. Chang, J.-L., A new method to calculate Franck-Condon factors of multidimensional harmonic oscillators including the Duschinsky effect. The Journal of chemical physics 2008, 128 (17), 174111.

144. Reutt, J.; Wang, L.; Lee, Y.-T.; Shirley, D., Molecular beam photoelectron spectroscopy and femtosecond intramolecular dynamics of $\mathrm{H}_{2} \mathrm{O}^{+}$and $\mathrm{D}_{2} \mathrm{O}^{+}$. The Journal of chemical physics 1986, 85 (12), 6928-6939.

145. Riedle, E.; Neusser, H.; Schlag, E., Electronic spectra of polyatomic molecules with resolved individual rotational transitions: Benzene. The Journal of Chemical Physics 1981, 75 (9), 4231-4240.

146. Small, G. J., Herzberg-Teller vibronic coupling and the Duschinsky effect. The Journal of Chemical Physics 1971, 54 (8), 3300-3306.

147. Warren, J. A.; Hayes, J. M.; Small, G. J., Symmetry reduction-vibronically induced mode mixing in the $S_{1}$ state of $\beta$-methylnaphthalene. The Journal of chemical physics 1984, 80 (5), 1786-1790.

148. Aschmann, S. M.; Chew, A. A.; Arey, J.; Atkinson, R., Products of the gas-phase reaction of $\mathrm{OH}$ radicals with cyclohexane: reactions of the cyclohexoxy radical. The Journal of Physical Chemistry A 1997, 101 (43), 8042-8048.

149. Orlando, J. J.; Iraci, L. T.; Tyndall, G. S., Chemistry of the cyclopentoxy and cyclohexoxy radicals at subambient temperatures. The Journal of Physical Chemistry A 2000, 104 (21), 5072-5079.

150. Zhang, L.; Callahan, K. M.; Derbyshire, D.; Dibble, T. S., Laser-induced fluorescence spectra of 4-methylcyclohexoxy radical and perdeuterated 
cyclohexoxy radical and direct kinetic studies of their reactions with $\mathrm{O}_{2}$. The Journal of Physical Chemistry A 2005, 109 (41), 9232-9240.

151. Zhang, L.; Kitney, K. A.; Ferenac, M. A.; Deng, W.; Dibble, T. S., LIF spectra of cyclohexoxy radical and direct kinetic studies of its reaction with $\mathrm{O}_{2}$. The Journal of Physical Chemistry A 2004, 108 (3), 447-454.

152. Jochnowitz, E. B.; Zhang, X.; Nimlos, M. R.; Flowers, B. A.; Stanton, J. F.; Ellison, G. B., Infrared Spectrum of the Propargyl Peroxyl Radical, $\mathrm{HC} \equiv \mathrm{C}-\mathrm{CH}_{2} \mathrm{OO}$

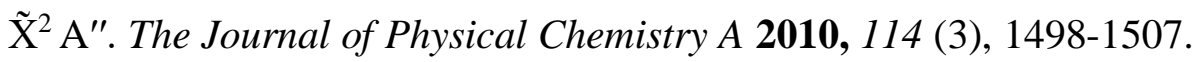

153. Jochnowitz, E. B.; Zhang, X.; Nimlos, M. R.; Varner, M. E.; Stanton, J. F.; Ellison, G. B., Propargyl Radical: Ab Initio Anharmonic Modes and the Polarized Infrared Absorption Spectra of Matrix-Isolated $\mathrm{HCCCH}_{2}$. The Journal of Physical Chemistry A 2005, 109 (17), 3812-3821.

154. Thomas, P. S.; Kline, N. D.; Miller, T. A., $\tilde{A}-\tilde{X}$ Absorption of propargyl peroxy radical $\left(\mathrm{H}-\mathrm{C} \equiv \mathrm{C}-\mathrm{CH}_{2} \mathrm{OO} \cdot\right)$ : A cavity ring-down spectroscopic and computational study. The Journal of Physical Chemistry A 2010, 114 (47), 12437-12446.

155. Crounse, J. D.; Paulot, F.; Kjaergaard, H. G.; Wennberg, P. O., Peroxy radical isomerization in the oxidation of isoprene. Physical Chemistry Chemical Physics 2011, 13 (30), 13607-13613.

156. Teng, A. P.; Crounse, J. D.; Wennberg, P. O., Isoprene peroxy radical dynamics. Journal of the American Chemical Society 2017, 139 (15), 5367-5377.

157. Lu, T.; Chen, F., Multiwfn: A Multifunctional Wavefunction Analyzer. Journal of Computational Chemistry 2012, 33 (5), 580-592. 
158. Lee, J.; Bozzelli, J. W., Thermochemical and kinetic analysis of the allyl radical with $\mathrm{O}_{2}$ reaction system. Proceedings of the Combustion Institute 2005, 30 (1), 1015-1022.

159. Pushkarsky, M. B.; Zalyubovsky, S. J.; Miller, T. A., Detection and characterization of alkyl peroxy radicals using cavity ringdown spectroscopy. The Journal of Chemical Physics 2000, 112 (24), 10695-10698.

160. Wu, S.; Dupré, P.; Miller, T. A., High-resolution IR cavity ring-down spectroscopy of jet-cooled free radicals and other species. Physical Chemistry Chemical Physics 2006, $8(14), 1682-1689$.

161. Dupré, P.; Miller, T. A., Quasi-fourier-transform limited, scannable, high energy titanium-sapphire laser source for high resolution spectroscopy. Review of scientific instruments 2007, 78 (3), 033102. 
Dispersed Fluorescence Spectroscopy of Jet-Cooled Isobutoxy and 2-Methyl-1-butoxy

\section{Radicals}

Author: Md. Asmaul Reza, Anam C. Paul, Neil J. Reilly, et al

Publication: The Journal of Physical Chemistry A

Publisher: American Chemical Society

Date: Sep 1, 2016

Copyright @ 2016, American Chemical Society

\section{PERMISSION/LICENSE IS GRANTED FOR YOUR ORDER AT NO CHARGE}

This type of permission/license, instead of the standard Terms \& Conditions, is sent to you because no fee is being charged for your order. Please note the following:

- Permission is granted for your request in both print and electronic formats, and translations.

- If figures and/or tables were requested, they may be adapted or used in part.

- Please print this page for your records and send a copy of it to your publisher/graduate school.

- Appropriate credit for the requested material should be given as follows: "Reprinted (adapted) with permission from (COMPLETE REFERENCE CITATION). Copyright (YEAR) American Chemical Society." Insert appropriate information in place of the capitalized words.

- One-time permission is granted only for the use specified in your request. No additional uses are granted (such as derivative works or other editions). For any other uses, please submit a new request. 
Laser-Induced Fluorescence and Dispersed Fluorescence Spectroscopy of Jet-Cooled Isopentoxy Radicals

Author: Md Asmaul Reza, Anam C. Paul, Neil J. Reilly, et al

Publication: The Journal of Physical Chemistry A

Publisher: American Chemical Society

Date: Oct 1, 2019

Copyright @ 2019, American Chemical Society

\section{PERMISSION/LICENSE IS GRANTED FOR YOUR ORDER AT NO CHARGE}

This type of permission/license, instead of the standard Terms \& Conditions, is sent to you because no fee is being charged for your order. Please note the following:

- Permission is granted for your request in both print and electronic formats, and translations.

- If figures and/or tables were requested, they may be adapted or used in part.

- Please print this page for your records and send a copy of it to your publisher/graduate school.

- Appropriate credit for the requested material should be given as follows: "Reprinted (adapted) with permission from (COMPLETE REFERENCE CITATION). Copyright (YEAR) American Chemical Society." Insert appropriate information in place of the capitalized words.

- One-time permission is granted only for the use specified in your request. No additional uses are granted (such as derivative works or other editions). For any other uses, please submit a new request. 
Dispersed Fluorescence Spectroscopy of Jet-Cooled 2-, 3-, and 4-Methylcyclohexoxy Radicals

Author: Jahangir Alam, Md. Asmaul Reza, Amy Mason, et al

Publication: The Journal of Physical Chemistry A

Publisher: American Chemical Society

Date: Jun 1, 2015

Copyright $@$ (2015, American Chemical Society

\section{PERMISSION/LICENSE IS GRANTED FOR YOUR ORDER AT NO CHARGE}

This type of permission/license, instead of the standard Terms \& Conditions, is sent to you because no fee is being charged for your order. Please note the following:

- Permission is granted for your request in both print and electronic formats, and translations.

- If figures and/or tables were requested, they may be adapted or used in part.

- Please print this page for your records and send a copy of it to your publisher/graduate school.

- Appropriate credit for the requested material should be given as follows: "Reprinted (adapted) with permission from (COMPLETE REFERENCE CITATION). Copyright (YEAR) American Chemical Society." Insert appropriate information in place of the capitalized words.

- One-time permission is granted only for the use specified in your request. No additional uses are granted (such as derivative works or other editions). For any other uses, please submit a new request. 


\section{ACSPublications}

Most Trusted. Most Cited. Most Read.

\section{Room-Temperature Cavity Ring-Down Spectroscopy of Methylallyl Peroxy Radicals}

Author: Md Asmaul Reza, Hamzeh Telfah, Raobo Xu, et al

Publication: The Journal of Physical Chemistry A

Publisher: American Chemical Society

Date: Apr 1, 2019

Copyright @ 2019, American Chemical Society

\section{PERMISSION/LICENSE IS GRANTED FOR YOUR ORDER AT NO CHARGE}

This type of permission/license, instead of the standard Terms \& Conditions, is sent to you because no fee is being charged for your order. Please note the following:

- Permission is granted for your request in both print and electronic formats, and translations.

- If figures and/or tables were requested, they may be adapted or used in part.

- Please print this page for your records and send a copy of it to your publisher/graduate school.

- Appropriate credit for the requested material should be given as follows: "Reprinted (adapted) with permission from (COMPLETE REFERENCE CITATION). Copyright (YEAR) American Chemical Society." Insert appropriate information in place of the capitalized words.

- One-time permission is granted only for the use specified in your request. No additional uses are granted (such as derivative works or other editions). For any other uses, please submit a new request. 


\title{
CURRICULUM VITAE
}

\author{
Md Asmaul Reza \\ e-mail: rezaasmaul@gmail.com
}

\section{EDUCATION}

University of Louisville, Louisville, KY

October 2019

$\mathrm{PhD}$ in Chemistry

Dissertation: Laser spectroscopic investigations of organic alkoxy and peroxy radicals

Supervisor: Dr. Jinjun Liu

University of Louisville, Louisville, KY

December 2015

Master of Science, Chemistry

University of Dhaka, Dhaka, Bangladesh

March 2012

Master of Science, Chemistry

Thesis: Studies of physicochemical properties of binary liquid mixtures at five different temperature 
Bachelor of Science, Chemistry

\section{PUBLICATIONS}

9. Koncz, B., Bazsó, G., Reza, M. A., Telfah, H., Hegedüs, K., Liu, J.; Tarczaya, G., Revealing Long-Range Substituent Effects in the Laser-Induced Fluorescence and Dispersed Fluorescence Spectra of Jet-cooled $\mathrm{CHxF}_{3}-\mathrm{xCH}_{2} \mathrm{O}(\mathrm{x}=1,2,3)$ Radicals. J. Phys. Chem. A (submitted).

8. Reza, M.A., Paul, A.C., Reilly, N.J., and Liu, J., Laser-Induced Fluorescence and Dispersed Fluorescence Spectroscopy of Jet-Cooled Isopentoxy Radicals. J. Phys. Chem. A, 2019, 123, 39, 8441-8447.

7. Paul, A.C., Sharma, K., Reza, M.A., Telfah, H., Miller, T. A., and Liu, J. Laser-Induced Fluorescence and Dispersed Fluorescence Spectroscopy of the $\tilde{A}^{2} \mathrm{E} \leftarrow \tilde{X}^{2} \mathrm{~A}_{1}$ Transition of Jet-Cooled Calcium Methoxide $\left(\mathrm{CaOCH}_{3}\right)$ Radicals. J. Chem. Phys. 2019, 151, 134303.

6. Reza, M. A., Telfah, H., Xu, R., and Liu, J., Room-Temperature Cavity Ring-Down Spectroscopy of Methylallyl Peroxy Radicals. J. Phys. Chem. A 2019 123, 16, 3510-3517.

5. Telfah, H., Reza, M. A., Alam, J., Paul, A. C., and Liu, J., Direct Observation of Tetrahydrofuranyl and Tetrahydropyranyl Peroxy Radicals via Cavity Ring-Down Spectroscopy. J. Phys. Chem. Lett. 2018 9, 4475-4480

4. Paul, A.C., Reza, M.A., and Liu, J., Dispersed-fluorescence spectroscopy of jet-cooled calcium ethoxide radical $\left(\mathrm{CaOC}_{2} \mathrm{H}_{5}\right)$. J. Mol. Spectrosc. 2016, 330,142-146 
3. Reza, M.A., Paul, A.C., Reilly, N.J., Alam, J. and Liu, J., Dispersed Fluorescence Spectroscopy of Jet-Cooled Isobutoxy and 2-Methyl-1-butoxy Radicals. J. Phys. Chem. A 2016, 120, 6761-6767

2. Alam, J., Reza, M.A., Mason, A., Reilly, N.J. and Liu, J., Dispersed Fluorescence Spectroscopy of Jet-Cooled 2-, 3-, and 4-Methylcyclohexoxy Radicals. J. Phys. Chem. A 2015, 119, 6257-6268

1. Wolinska, J.J., Garabato, B.D., Alam, J., Reza, A., Kozlowski, P.M., Structural and electronic properties of an $\left[\left(\mathrm{Al}_{2} \mathrm{O}_{3}\right)_{4}\right]^{+}$cluster. J. of Mol. Model. 2015, 21 (7), 170

\section{CONFERENCE PRESENTATIONS}

7. Md Asmaul Reza, Hamzeh Telfah, Anam C. Paul, Jahangir Alam, Jinjun Liu. Cavity Ring-down Spectroscopy of 1-, 2- and 3-methylallyl peroxy radicals. $73^{\text {rd }}$ International Symposium on Molecular Spectroscopy, Champaign-Urbana, Illinois, USA. June 2018

6. Hamzeh Telfah, Md Asmaul Reza, Anam C. Paul, Jinjun Liu. Observation of The $\tilde{A} \leftarrow \tilde{X}$ Electronic Transitions of Tetrahydropyranyl and Tetrahydrofuranyl Peroxy Radicals by Room-Temperature Cavity Ring-Down Spectroscopy. $73^{\text {rd }}$ International Symposium on Molecular Spectroscopy Champaign-Urbana, Illinois, USA. June 2018

5. Anam C. Paul, Md Asmaul Reza, Ketan Sharma, Terry A. Miller, Jinjun Liu, Alkaline Earth Monoalkoxide Free Radicals as Candidates for Laser Cooling of Poly-Atomic Molecules. $73^{\text {rd }}$ International Symposium on Molecular Spectroscopy, ChampaignUrbana, Illinois, USA. June 2018 
4. Anam C. Paul, Md Asmaul Reza, Pranoy Deb Shuvra, Jinjun Liu. Laser-Induced Fluorescence and Dispersed-Fluorescence Spectroscopy of Jet-Cooled Calcium Monoalkoxide Radicals. $73^{\text {rd }}$ International Symposium on Molecular Spectroscopy, Champaign-Urbana, Illinois, USA. June 2018

3. Anam C. Paul, Md Asmaul Reza, Jinjun Liu, Dispersed-Fluorescence Spectroscopy of Jet-Cooled Calcium Ethoxide Radical $\left(\mathrm{CaOC}_{2} \mathrm{H}_{5}\right) .70^{\text {th }}$ International Symposium on Molecular Spectroscopy, Champaign-Urbana, Illinois, USA. June 2016

2. Md Asmaul Reza, Neil J Reilly, Jahangir Alam, Amy Mason, Jinjun Liu. Dispersed Fluorescence Spectroscopy of Jet-Cooled Isobutoxy, 2-Methyl-1-butoxy and Isopentoxy Radicals. $69^{\text {th }}$ International Symposium on Molecular Spectroscopy, Champaign-Urbana, Illinois, USA June 2015

1. Jahangir Alam, Md Asmaul Reza, Amy Mason, Jinjun Liu. Dispersed Fluorescence Spectroscopy of Jet-Cooled Methylcyclohexoxy Radicals. $69^{\text {th }}$ International Symposium on Molecular Spectroscopy, Champaign-Urbana, Illinois, USA. June 2015 